Aus der Klinik für Unfallchirurgie, Orthopädie und Plastische Chirurgie (Prof. Dr. med. W. Lehmann)

der Medizinischen Fakultät der Universität Göttingen

\title{
Komplikationen und Komplikationsrisiken bei der Versorgung kindlicher Femurschaftfrakturen
}

Statistische Analyse an den Traumazentren der Universitätsmedizin Göttingen und Magdeburg

\author{
INAUGURAL-DISSERTATION \\ zur Erlangung des Doktorgrades \\ der Medizinischen Fakultät der \\ Georg-August-Universität zu Göttingen
}

vorgelegt von

Maria Rita Klauser

aus

Berlin

Göttingen 2017 
Dekan:

Prof. Dr. rer. nat. H. K. Kroemer

Referent:

Ko-Referent:
Prof. Dr. med. S. Sehmisch

Prof. Dr. med. E. Wilichowski

Datum der mündlichen Prüfung: 27.06.2019 
Hiermit erkläre ich, die Dissertation mit dem Titel "Komplikationen und Komplikationsrisiken bei der Versorgung kindlicher Femurschaftfrakturen - Statistische Analyse an den Traumazentren der Universitätsmedizin Göttingen und Magdeburg" eigenständig angefertigt und keine anderen als die von mir angegebenen Quellen und Hilfsmittel verwendet zu haben.

Göttingen, den 01.09.2017 



\section{Inhaltsverzeichnis}

Inhaltsverzeichnis I

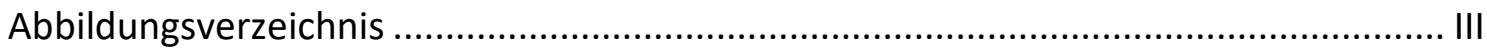

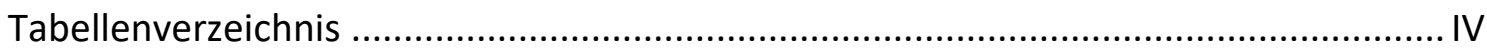

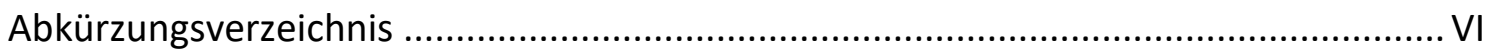

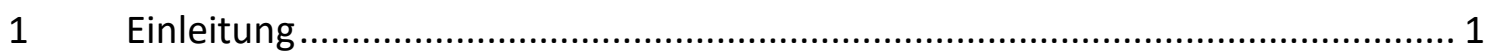

1.1 Untersuchungsgegenstand, Fragestellung und Zielsetzung ....................................1

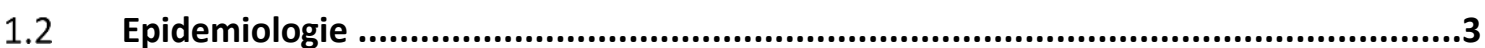

1.2.1 Häufigkeit von kindlichen Frakturen und Femurschaftfrakturen im

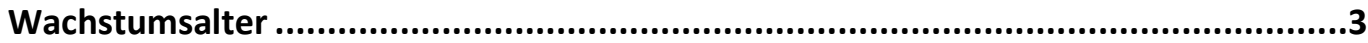

1.2.2 Ursachen von kindlichen Verletzungen sowie Femurschaftfrakturen im

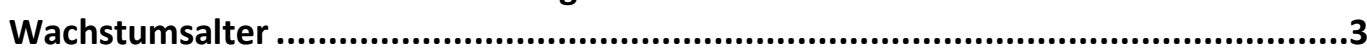

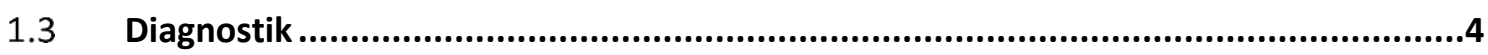

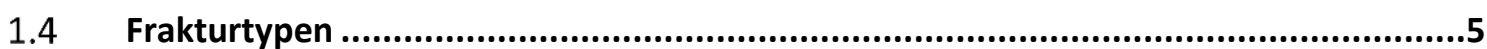

1.4.1 Bruchmuster von Schaftfrakturen: Biegungs-, Quer-, Schräg-, Spiral-, Mehrfragment-, und Trümmerfraktur .....................................................................7

1.4.2 Klassifikationen für Frakturen im Wachstumsalter...............................................8

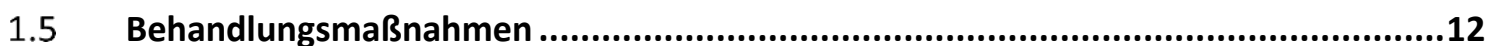

1.5.1 Konservative Therapie der Femurschaftfraktur.......................................................14

1.5.2 Operative Therapie der kindlichen Femurschaftfraktur .........................................18

1.6 Ziele der Behandlung der kindlichen Femurschaftfraktur und Prognose ...................26

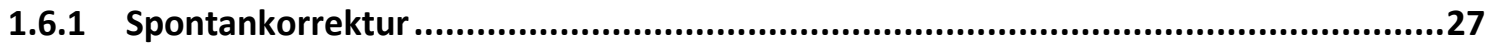

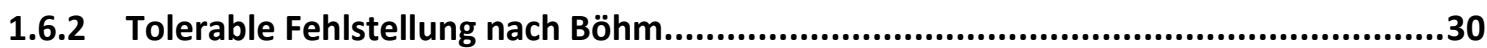

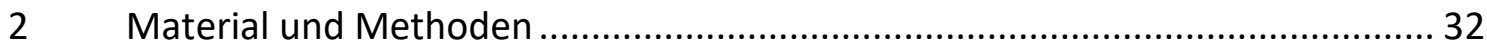

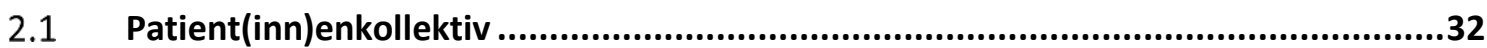

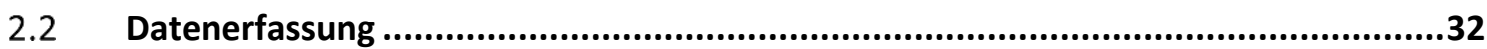

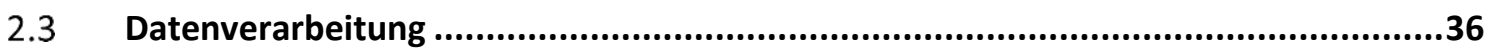

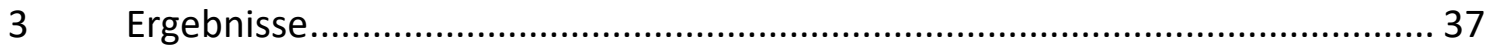

3.1 Patient(inn)enkollektiv: Geschlecht, Alter, Body-Mass-Index, Perzentile,

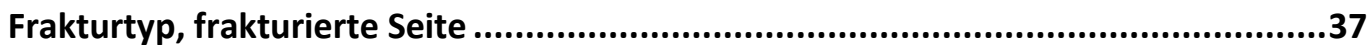

3.2 Kontext des Frakturereignisses: Unfallgeschehen, Begleiterkrankungen,

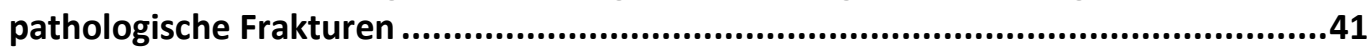

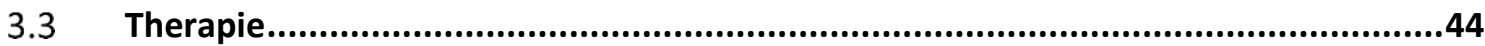


3.4 Komplikationen: intra- und postoperative Komplikationen, Komplikationsformen, mögliche Einflussfaktoren, Verfahrenswechsel, Fehlstellung nach Böhm .49

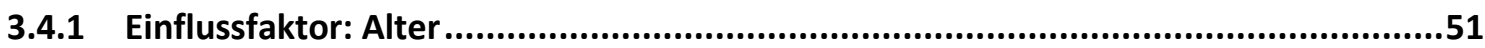

3.4.2 Einflussfaktoren: Body-Mass-Index, Perzentilkurven ........................................53

3.4.3 Einflussfaktor auf Komplikationen: Frakturtyp ...................................................54

3.4.4 Einflussfaktoren: Art der Versorgung, Repositionsform........................................55

3.4.5 Einflussfaktoren: Reposition und Frakturform, Material und Frakturform ...................56

3.4.6 Einflussfaktor: Verhältnis Markraumdurchmesser zu ESIN-Drahtdicke .......................58

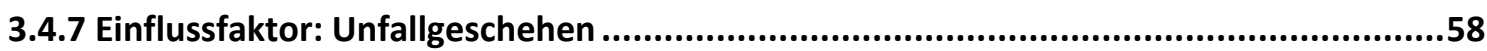

3.4.8 Einflussfaktor: Zeitraum zwischen Operation und Metallentfernung ..........................59

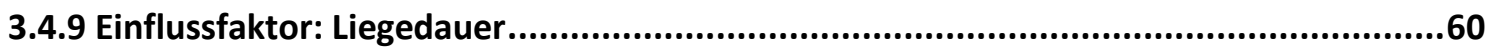

3.4.10 Einflussfaktor: pathologische Frakturen, Begleiterkrankungen ................................60

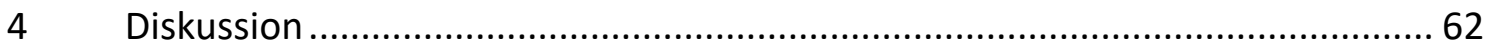

4.1 Quantität und Qualität der Komplikationen im Patient(inn)enkollektiv...................62

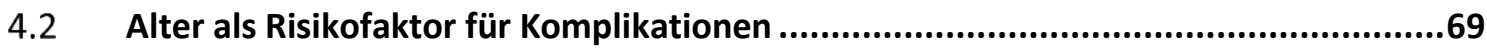

4.3 Verhältnis von Markraumdurchmesser zu Drahtdicke als Risikofaktor für Komplikationen bei der ESIN-Therapie ..............................................................73

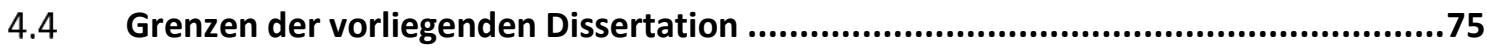

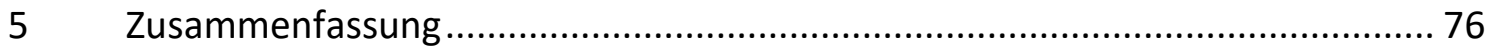

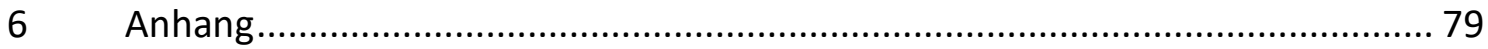

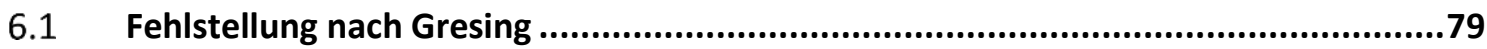

6.2 Pädiatrische Frakturklassifikation für lange Röhrenknochen nach Slongo ..................79

6.3 Weiterführende Übersichten und Tabellen zu Kapitel 3 .........................................82

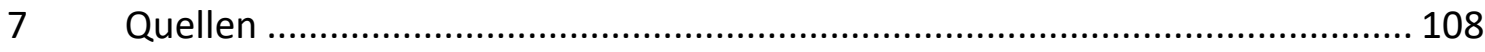

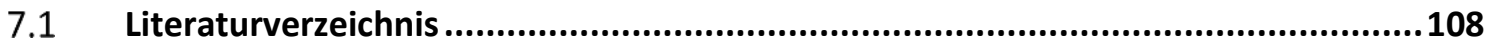

7.2 Bild-, Internet-, Tabellen- und sonstige Quellen .....................................................115 


\section{Abbildungsverzeichnis}

Abbildung 1: Frakturformen und Einteilung von Knochenabschnitten ..................................7

Abbildung 2: Übersicht Li-La Klassifikation Version 2 ........................................................11

Abbildung 3: Vorgedruckte Quadrate zur korrekten Identifikation der Metaphyse ..................11

Abbildung 4: Einteilung der Schaftfrakturen gemäß Li-La Klassifikation ..................................12

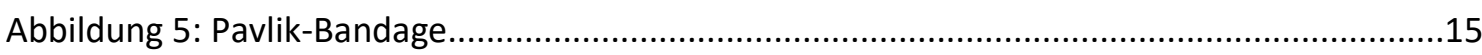

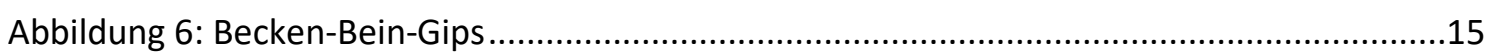

Abbildung 7: Proximale Femurschaftfraktur bei einem siebenjährigen Mädchen .....................15

Abbildung 8 und 9 : Kinder in Behandlung mit Overhead-Extension .......................................16

Abbildung 10: Querfraktur eines vier Monate alten Säuglings.............................................17

Abbildung 11: Retro- und anterograde Nagelung einer Querfraktur mit ESINs .......................19

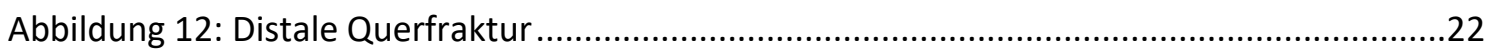

Abbildung 13: Distale Femurschaftfraktur versorgt mit drei ESINs .......................................22

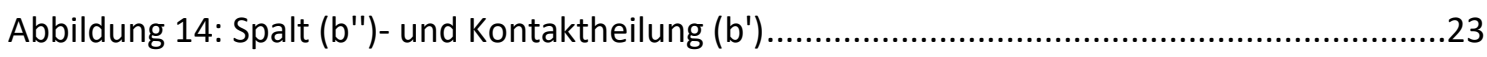

Abbildung 15: Femurschaftfraktur eines neunjährigen Jungen..............................................24

Abbildung 16: Femurschaftfraktur eines dreijährigen Jungen .............................................25

Abbildung 17: Korrektur einer Seit-zu-Seit Fehlstellung.......................................................28

Abbildung 18: Kombination Mechanismen Spontankorrektur ................................................28

Abbildung 19: Wachstumsanteil der einzelnen Fugen .....................................................28

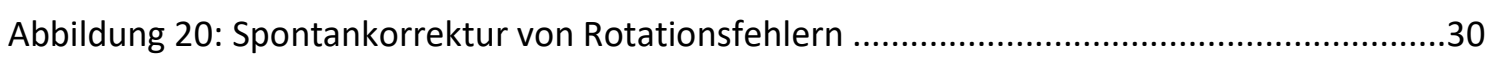

Abbildung 21: Mögliche Folgen von stimulativen Wachstumsstörungen .................................31

Abbildung 22: Altersverteilung im Patient(inn)enkollektiv in 2-Jahres-Abständen...................37

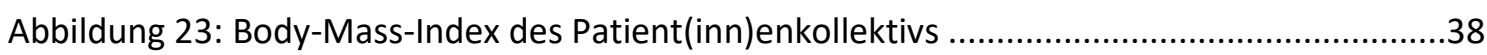

Abbildung 24: Anzahl Patient(inn)en pro Perzentilkurve für den Body-Maß-Index ....................38

Abbildung 25: Verteilung Gewicht im Patient(inn)enkollektiv .................................................39

Abbildung 26: Häufigkeiten der verschiedenen Frakturformen ............................................40

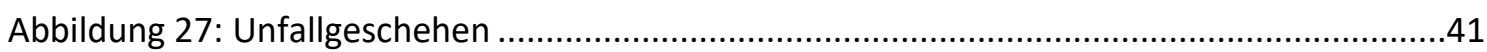

Abbildung 28: Häufigkeit von pathologischen Frakturen im Patient(inn)enkollektiv.................44

Abbildung 29: Häufigkeit von Begleiterkrankungen im Patient(inn)enkollektiv ........................44

Abbildung 30: Arten der Reposition............................................................................45

Abbildung 31: Verwendetes Material bei der Reposition im Falle einer Operation...................45

Abbildung 32: Verweildauer der Patient(inn)en im Krankenhaus ...........................................46

Abbildung 33: Durchmesser der Markhöhle proximal des Frakturspalts ................................47

Abbildung 34: Durchmesser der Markhöhle distal des Frakturspalts....................................47

Abbildung 35: Verhältnis Markraumdurchmesser zur Dicke der ESINs.................................47

Abbildung 36: Zeitlicher Abstand zwischen Operation und Metallentfernung .........................48

Abbildung 37: Häufigkeit von intraoperativen Komplikationen ...........................................49

Abbildung 38: Häufigkeit der posttherapeutischen Komplikationen ....................................49

Abbildung 39: Behandlung der Patient(inn)en mit Komplikationen........................................49

Abbildung 40: Arten der postoperativen Komplikationen......................................................50 
Abbildung 41: Komplikationswahrscheinlichkeit im jeweiligen Lebensjahr .52

Abbildung 42: Einfluss des Body-Mass-Index auf die postoperative Komplikationsrate .53

Abbildung 43: Lokale polynomiale Kernel-Regression: Zusammenhang zwischen Komplikationen und dem Verhältnis Markraumdurchmesser zu Drahtdicke .58

Abbildung 44: Kernel-Regression von postoperativen Komplikationen und des Zeitraumes zwischen Metallimplantation und -entfernung. ...................................................60

Abbildung 45: Einteilung und Nummerierung der Knochen nach der AO-PCCF ......................81

Abbildung 46: Einteilung von diaphysären Frakturen der AO-PCCF .....................................81

\section{Tabellenverzeichnis}

Tabelle 1: Gruppen der Schaft- und Gelenkfrakturen ..9

Tabelle 2: Grenzwerte für "tolerabel" dislozierte Achsabweichungen nach von Laer ................31

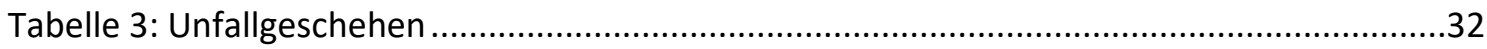

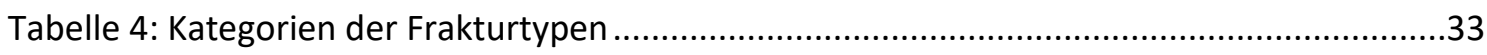

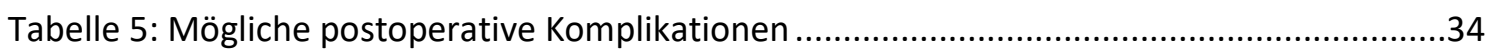

Tabelle 6: Analysedaten und Dokumente der Patient(inn)enakten ......................................35

Tabelle 7: Häufigkeiten Frakturtypen und Komplikationswahrscheinlichkeit ...........................54

Tabelle 8: Wahrscheinlichkeit für Komplikationen bei konservativer Behandlung ....................55

Tabelle 9: Wahrscheinlichkeit für Komplikationen bei offener operativer Behandlung .............55

Tabelle 10: Komplikationswahrscheinlichkeit offene und geschlossene Operation ...................56

Tabelle 11: Reposition bei den jeweiligen Frakturtypen ..................................................57

Tabelle 12: Komplikationswahrscheinlichkeit bei pathologischen Frakturen. .........................61

Tabelle 13: Komplikationsrisiko mit und ohne Begleiterkrankungen ....................................61

Tabelle 14: Akzeptable Fehlstellungen am Femurschaft ..............................................79

Tabelle 15: Häufigkeiten des Unfallgeschehens je nach BMI der Patient(inn)en.....................82

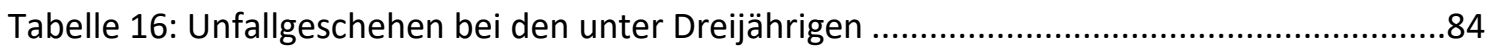

Tabelle 17: Unfallgeschehen bei den Drei- bis Achteinhalbjährigen .....................................85

Tabelle 18: Unfallgeschehen bei den über Achteinhalbjährigen ..........................................86

Tabelle 19: Frakturtypen und die jeweiligen Unfallgeschehen............................................87

Tabelle 20: Häufigkeiten der Frakturtypen bei den jeweiligen Unfallgeschehen......................89

Tabelle 21: Art der Versorgung der jeweiligen Frakturtypen .............................................91

Tabelle 22: Multivariablen-Regression von Komplikationen und Frakturtyp ..........................93

Tabelle 23: Häufigkeiten der Frakturtypen und jeweilige Versorgungsart...............................94

Tabelle 24: Komplikationswahrscheinlichkeit im jeweiligen Alter .....................................95

Tabelle 25: Multi-Variablen-Modell: Alter, Drahtverhältnisse und Komplikationen ..................96

Tabelle 26: Altersgruppen im Vergleich und ihr jeweiliges Komplikationsrisiko .......................96

Tabelle 27: Frakturtypen bei Kindern unter drei Jahren..................................................97

Tabelle 28: Frakturtypen der drei- bis achteinhalbjährigen Patient(inn)en ............................97

Tabelle 29: Frakturtypen der über achteinhalbjährigen Patient(inn)en.................................98

Tabelle 30: Multi-Variablen-Modell: BMI, Alter und postoperative Komplikationen ..................99 
Tabelle 31: logistische Regression: Komplikationen und Perzentilkurven ..............................100

Tabelle 32: t-Test: Wahrscheinlichkeit von Komplikationen bei konservativer Therapie. .........100

Tabelle 33: t-Test: Wahrscheinlichkeit von Komplikationen bei offener Operation gegenüber allen anderen Behandlungsmethoden

Tabelle 34: t-Test: Wahrscheinlichkeit von Komplikationen bei offener Operation gegenüber geschlossener Operation

Tabelle 35: Regression von Komplikationswahrscheinlichkeit, Frakturtyp und Art der Operation

Tabelle 36: Regression von Komplikationen, Frakturtyp und Implantationsmaterial 103

Tabelle 37: Logistische Regression: Verhältnis Markraumdurchmesser zu ESIN-Drahtdicke und das Risiko für Komplikationen. .104

Tabelle 38: Häufigkeiten von Komplikationen bei den jeweiligen Unfallgeschehen 1044

Tabelle 39: Multivariablen-Regression von Alter, Unfallmechanismus und Komplikationen ...106

Tabelle 40: Quadratische Regression von Komplikationsrate und Dauer zwischen

Metallimplantation und -entfernung .106

Tabelle 41: Quadratische Regression des Komplikationsrisikos und der Liegedauer... .107

Tabelle 42: t-Test: Wahrscheinlichkeit von Komplikationen bei pathologischen Frakturen .....107 


\section{Abkürzungsverzeichnis}

AAOS American Association of Orthopaedic Surgeons

ALFN Adolescent Lateral Femoral Nail (Adoleszentennagel)

AO Arbeitsgemeinschaft für Osteosynthesefragen

AWMF Arbeitsgemeinschaft der Wissenschaftlichen Medizinischen Fachgesellschaften e.V.

BMI Body-Mass-Index

bspw. beispielsweise

DGU Deutsche Gesellschaft für Unfallchirurgie

ESIN elastisch stabiler intramedullärer Nagel (Federnagel) bzw. Nagelung

inkl. Inklusiv(e)

Li-La Licht und Lachen für kranke Kinder. Effizienz in der Medizin e.V.

PCCF Pediatric Comprehensive Classification of Long-Bone Fractures

S1-Leitlinie Stufenklassifikation 1 der AWMF

TEN-Set Titanium Elastic Nail System 


\section{$1 \quad$ Einleitung}

\subsection{Untersuchungsgegenstand, Fragestellung und Zielsetzung}

Gegenstand der vorliegenden Arbeit sind Oberschenkelschaftfrakturen im Wachstumsalter. Sie machen bis zu 1,5\% der kindlichen Frakturen aus und stellen die zweithäufigsten diaphysären Knochenbrüche an der unteren Extremität dar (Dietz und Schlickewei 2011). Femurschaftfrakturen sind bei Kindern von besonderer Bedeutung, da sie einen gravierenden Einschnitt in der Krankheitserfahrung eines Kindes darstellen. Dies geht mit einer zum Teil mehrwöchigen Therapie, eventuell mehreren Operationen, Krankenhausaufenthalten und einer Immobilisation einher, die das Kind aus dem gewohnten Umfeld und sozialen Aktivitäten reißen. Die Fraktur im Wachstumsalter hat eine meist hervorragende Prognose auf Ausheilung und besonders junge Kinder brauchen nicht mit Spätkomplikationen wie Achsen-, Rotationsfehlern oder Beinlängendiskrepanzen zu rechnen. Kommt es jedoch zu solchen Komplikationen, hat dies Auswirkungen auf die Haltung des Beckens und die Wirbelsäulenstatik des heranwachsenden Kindes. Durch einen Beckenschiefstand kann es zu einer kompensatorischen Skoliose kommen oder einem prä-arthrotischen Hüftgelenksschaden. Die Behandelnden stehen vor der Herausforderung, Patient(inn)en die Therapie zukommen zu lassen, die das für sie geringste Komplikationsrisiko birgt und gleichzeitig unnötige Belastungen (z.B. Krankenhausaufenthalt, Schmerzen, aufwendige Pflege) für Kind und Eltern vermeidet.

In den vergangenen 30 Jahren gab es einen Wandel von der konservativen hin zur operativen Therapie. Bevorzugt wird heute die elastisch stabile intramedulläre Nagelung (ESIN) - bei 90\% der Patient(inn)en wird diese Technik im Falle einer Operation angewendet (Dietz und Schlickewei 2011).

Die Leitlinien der Deutschen Gesellschaft für Kinderchirurgie (S1-AWMF-Leitlinie 006/016 von 09/2014 ${ }^{1}$ ) empfehlen bei Femurschaftfrakturen eine konservative Behandlung mit einem Beckenbeingips oder der Overhead-Extension für Kinder unter drei Jahren. Ab dem dritten Lebensjahr sollte die operative ESIN-Versorgung vorgenommen werden oder im Falle einer drittgradig offenen Fraktur, eines Polytraumas oder einer Trümmerfraktur ein Fixateur externe für die primäre Stabilisierung genutzt werden. Außer dem Alter und der Schwere der Verletzung(en)

\footnotetext{
${ }^{1}$ http://www.awmf.org/leitlinien/detail/II/006-016.html, aufgerufen am 15.08.2016
} 
ist das Gewicht des Kindes limitierender Faktor für eine Nagelung mit elastisch stabilen Federnägeln. So wird für Kinder, die mehr als 50 kg wiegen, auf den Adoleszentennagel verwiesen.

Im Zentrum dieser Arbeit stehen die Analyse und Erörterung von möglichen Einflussfaktoren auf das Komplikationsrisiko bei der Behandlung von kindlichen Femurschaftfrakturen. Im Rahmen einer Datenerhebung an zwei deutschen Traumazentren (Universitätsklinika Göttingen und Magdeburg) soll die Versorgungsrealität abgebildet und die Rolle von möglichen Kriterien diskutiert werden: z.B. Alter, Gewicht, Geschlecht, Frakturtyp, Art der Versorgung, Unfallhergänge, Verhältnis Markraum- zu Nageldurchmesser bei der ESIN-Therapie. Ein weiterer Schwerpunkt dieser Arbeit besteht darin, die Anzahl und die Natur der Komplikationen aufzuzeichnen sowie die zu Grunde liegenden Zusammenhänge zu untersuchen und zu erörtern. Nicht zuletzt ist es Ziel der Analysen, dazu beizutragen, dass bei künftigen Behandlungen Komplikationen vermieden werden können.

Konkret stellt sich die Frage, ob die Hauptkriterien Alter und Gewicht bei der Entscheidung für eine bestimmte Therapie weiterhin den maßgeblichen Einfluss haben sollten. Zudem stellen sich folgende Fragen: Könnte man den unter Dreijährigen eine mehrwöchige Immobilisation ersparen, indem man auch ihnen eine operative Therapie zukommen lässt? Und welche der beiden konservativen Therapien ist komplikationsärmer? Sind womöglich andere Einflussfaktoren als Alter und Gewicht auszumachen, die das Komplikationsrisiko beeinflussen? Hat ein Kind das dritte Lebensjahr erreicht, muss abgewogen werden, welche der operativen Techniken zu empfehlen ist und welche Faktoren das Risiko für Komplikationen erhöhen.

Lascombes et al. (2013) stellen fest, dass die Nageldicke die Komplikationsrate im Falle einer Behandlung mit ESIN signifikant beeinflusst. So muss der Durchmesser des Femurmarkraums ausgemessen und für jeden Patienten/jede Patientin die passende Nagelstärke ausgewählt werden: Die Nageldicke sollte mehr als 40\% des Markraumsdurchmessers betragen. Im Falle eines Markraumdurchmessers breiter als $10 \mathrm{~mm}$ ist es ratsam, eine andere Methode zur Frakturversorgung für einen komplikationslosen Behandlungsverlauf zu wählen. Um diesen Aspekt bei den erwartbar häufigen ESINTherapien zu überprüfen und einen möglichen Risikofaktor auszumachen, wurden Röntgenbilder der mit ESIN behandelten Patient(inn)en ausgewertet. Es stellt sich die Frage, welches Verhältnis von Markraumdurchmesser zu Nageldicke im Patient(inn)enkollektiv vorliegt und ob dieses einen signifikanten Einfluss auf die Komplikationsrate hat. 


\subsection{Epidemiologie}

Die Prävalenz für eine Fraktur im Wachstumsalter für ein mitteleuropäisches Kind liegt bei $21-25 / 1000$ Kinder pro Jahr. In 62,7\% der Fälle werden lange Röhrenknochen frakturiert. Insgesamt tritt die Femurschaftfraktur mit 0,6 bis 1,5\% an der Gesamtheit der Knochenbrüche von Kindern eher selten auf (Kraus 2006; von Laer et al. 2007; Hedström et al. 2010; Schalamon et al. 2011; Dietz und Schlickewei 2011): Die Inzidenz beträgt jährlich 11-26/100`000 Kinder (jünger als 16-18 Jahre) (Hedström et al. 2010; Rewers et al. 2005; Poolman et al. 2006).

\subsubsection{Häufigkeit von kindlichen Frakturen und Femurschaftfrakturen im Wachstumsalter}

Jones et al. (2002) ermittelten, dass sich die Hälfte aller Kinder bis zum 18. Lebensjahr eine Fraktur zuzieht. Im Rahmen ihrer Untersuchung wurden Daten von 291 Patien$\mathrm{t}($ inn)en mit Frakturen von ihrem dritten bis zum 18. Lebensjahr analysiert. Die Kinder erlitten insgesamt 498 Frakturen in 15 Jahren. Das heißt, dass Patient(inn)en, die einmal einen Bruch erleiden, sich wahrscheinlicher im Laufe ihres Wachstums eine oder mehrere weitere Frakturen zuziehen im Vergleich zu Kindern, die noch keine Fraktur erlitten haben. Im Gegensatz dazu beobachteten Hedström et al. (2010) ein niedrigeres mittleres Risiko für eine Fraktur bis zum 17. Lebensjahr: es beträgt $34 \%$. Speziell zu Femurschaftfrakturen sei auf von Heideken et al. (2011) verwiesen: In ihrer retrospektiven Studie fanden sie eine sich um $42 \%$ verringernde Inzidenz über einen Zeitraum von 18 Jahren (1987-2005).

\subsubsection{Ursachen von kindlichen Verletzungen sowie Femurschaftfrakturen im Wachstumsalter}

In einer vierjährigen Monitoring-Studie registrierten Böhmann und Ellsäßer (2004) die Unfallhergänge aller Kinder, die in Notfalleinrichtungen in Delmenhorst vorgestellt und behandelt wurden. In 50\% der Fälle war der Mechanismus (unabhängig vom Alter) ein Sturz. Bis zum Schulalter stürzten die Patient(inn)en vom Wickeltisch, aus dem Kinderbett oder von Spielplatzgeräten, später vom Fahrrad und von Spielplatzgerüsten.

Ellsäßer (2006) ermittelte in einer epidemiologischen Studie die Mortalität, Unfallursachen und -orte von Kindern unter 15 Jahren in Deutschland. Sie stellt fest, dass unter Fünfjährige sich meistens im häuslichen Umfeld verletzten und Vier- bis Fünfzehnjährige am häufigsten in der Schule, Freizeit oder im Straßenverkehr verunglückten. 
Kahl et al. (2007) werteten die Daten des Kinder- und Jugendgesundheitssurveys (KiGGS) von 2003 bis 2006 aus. Zugrunde liegen Fragebögen von 16706 Eltern, die Auskünfte gaben zu Verletzungen der vergangenen zwölf Monate ihrer ein- bis siebzehnjährigen Kinder. Stürze sind in allen Altersgruppen der häufigste Unfallmechanismus: „Stürze in der Ebene (35,2\%), aus der Höhe (25,2\%), und Zusammenstöße (20,6\%)" (Kahl et al. 2007). Ein weiterer Aspekt ist der Zusammenhang zwischen Alter und Konsequenz des Unfalls. So war es wahrscheinlicher, dass Kinder einen Knochenbruch erlitten, je älter sie waren. Der Frakturanteil bei Kleinkindern lag bei $10,7 \%$ und erhöhte sich bei den 15- bis 17Jährigen auf $20,8 \%$.

Auch die Ursachen und Unfallhergänge, die zu Femurschaftfrakturen führen, unterscheiden sich je nach Alter der Kinder. Im Kleinkindalter bis zu vier Jahren verunfallen die meisten Patient(inn)en durch einen Sturz aus einer Höhe von unter einem oder bis zu drei Metern. Im Alter von vier bis zwölf Jahren erleiden sie Oberschenkelbrüche aktivitätsbedingt bei Sport- oder Freizeitaktivitäten. Ab der Pubertät spielen Hochrasanztraumata und Unfälle mit großer Krafteinwirkung auf den Knochen die größte Rolle - so erleiden Heranwachsende im Alter von 13 bis 18 Jahren Femurschaftfrakturen am häufigsten im Rahmen von Sport- und Verkehrsunfällen (von Heideken et al. 2011; Rewers et al. 2005). Misshandlungen stellen besonders eine Gefahr für die Jüngsten dar: Die Frakturrate liegt hier im ersten Lebensjahr bei 4,2 bis 30,5\% (von Heideken et al. 2011; Leventhal et al. 2008).

\subsection{Diagnostik}

Wegweisend ist die Klinik der Patient(inn)en: In den meisten Fällen sind sie gehunfähig, zudem treten schmerzhafte Bewegungseinschränkungen, lokale Schwellung, Deformierung, Verkürzung des frakturierten Beines und bzw. oder eine Rotationsfehlstellung auf.

Bei der Anamnese sollte unbedingt eruiert werden, ob das Trauma adäquat war. Je nach Unfallhergang ist das Kind auf mögliche Begleitverletzungen hin zu untersuchen (Gresing 2011; Maier et al. 2010; Weinberg und Schneidmüller 2010a). Besonders bei Säuglingen und Kleinkindern muss der Untersuchende bei einer widersprüchlichen Anamnese an Kindesmisshandlung denken. Nach Leventhal et al. (2008) liegt bei Kindern, die im ersten Lebensjahr eine Femurschaftfraktur erleiden, bei nahezu einem Drittel der Fälle eine nicht akzidentelle Ursache zugrunde. Mit dem Alter des Kindes sinkt die Wahrscheinlichkeit, dass es eine Fraktur im Rahmen einer Misshandlung erfährt. So geht man davon aus, dass nur noch $5 \%$ der Patient(inn)en jünger als drei 
Jahre eine Femurschaftfraktur durch Misshandlung zugefügt wird (Gresing 2011; Leventhal et al. 2008; Maier et al. 2010; von Heideken et al. 2011; Weinberg und Schneidmüller 2010b).

Die Standarddiagnostik ist eine Röntgenaufnahme in zwei Ebenen und den angrenzenden Gelenken unter Analgesie. Ist die Fraktur eindeutig disloziert, kann auf die zweite Aufnahme verzichtet oder sie z.B. im Falle einer Operation später unter Narkose nachgeholt werden.

\subsection{Frakturtypen}

Der Darstellung von Frakturtypen und deren Klassifikationen werden folgende drei Definitionen einer Fraktur zu Grunde gelegt:

1. „Eine Fraktur ist die Unterbrechung des Knochens in 2 oder mehrere durch einen Frakturspalt getrennte Bruchstücke." (Biberthaler und Oestern 2012).

2. „Wenn die Festigkeit des Knochengewebes einer einwirkenden Belastung nicht standhält, kommt es zum Knochenbruch.“ (Lüllmann-Rauch 2006)

3. „Eine Fraktur ist eine Kontinuitätsunterbrechung des Knochens, die mit Schmerzen und einem Funktionsverlust einhergeht." (Raschke und Haas 2012)

Frakturen werden unter verschiedenen Gesichtspunkten, wie z.B. Lokalisation, Ätiologie, Frakturform, Bruchmuster, Dislokationsgrad oder komplettes Verletzungsausmaß beschrieben und klassifiziert (siehe unten). Diese Einteilungen helfen in der kollegialen Kommunikation im klinischen Alltag, erleichtern die Indikationsstellung, tragen zur sicheren und korrekten Behandlung der Patient(inn)en bei und sind wichtig in der medizinischen Forschung sowie beim Erstellen bzw. Befolgen von Leitlinien.

Von besonderer Bedeutung für Frakturen im Wachstumsalter ist die Einteilung der Arbeitsgemeinschaft für Osteosynthese $(A O)$ gemäß Slongo und die Klassifikation für lange Röhrenknochen vom Verein „Licht und Lachen für kranke Kinder. Effizienz in der Medizin e.V.“ (Li-La Klassifikation). Siehe Unterkapitel 1.4.2.

Die oben genannten Gesichtspunkte werden im Folgenden erläutert:

Bei der Angabe der Bruchstelle (Lokalisation), unterscheidet man zwischen Gelenkfraktur (eine Gelenkfläche ist mit betroffen), Schaftfraktur (die Fraktur liegt im Bereich der Diaphyse) oder Etagenfraktur (ein Knochen weist mehrere Frakturstellen auf) (Müller 2011). Die nur im Kindesalter auftretenden Epiphysenfugenverletzungen werden nach Salter-Harris oder Aitken eingeteilt, sind jedoch nicht Gegenstand der Arbeit (von Laer 2010; Bibertahler und Oestern 2012). 
Um das zur Fraktur führende Ereignis zu beschreiben (Ätiologie), nimmt man die Unterteilung in direkte, indirekte und pathologische Fraktur sowie die Ermüdungsfraktur vor. Bei einer direkten Fraktur ist dem gesunden Knochen eine „adäquate äußere Gewalteinwirkung" vorausgegangen (Biberthaler und Oestern 2012; Raschke und Haas 2012), zum Beispiel Schlag mit Werkzeug auf einen Finger. Bei indirekter Krafteinwirkung hingegen entstehen durch Hebel- und Drehkraft Torsions- oder Biegungsbrüche (Beispiel: Unterschenkel-Spiralfraktur beim Skifahrer (Biberthaler und Oestern 2012; Raschke und Haas 2012). Erleidet der Patient/die Patientin einen Knochenbruch ohne ein adäquates Trauma, so spricht man von einer pathologischen Fraktur. Der Knochen war also schon vor dem Ereignis krankhaft verändert (Müller 2011; Biberthaler und Oestern 2012; Raschke und Haas 2012).

Die Angabe, ob eine Fraktur "offen“ oder "geschlossen“ ist (Frakturform), gibt Informationen zur Weichteilsituation der unmittelbaren Frakturumgebung und somit auch einen Hinweis zur Dringlichkeit der Versorgung (Biberthaler und Oestern 2012; Raschke und Haas 2012; Müller 2011).

Frakturen kann man außerdem in komplette und inkomplette Brüche einteilen. Zu den kompletten Brüchen zählen alle Frakturen, bei denen eine vollständige Knochendurchtrennung vorliegt (Biberthaler und Oestern 2012; Müller 2011). Beispiele für inkomplette Frakturen sind Fissuren, Knochenanrisse und die kindlichen Frakturen Grünholzfraktur, Bowing Fracture und Torusfraktur. Die Grünholzfraktur ist ein Biegungsbruch, bei dem die konvexseitige Kortikalis bricht und das Periost einreißt, die konkavseitige Kortikalis aber nur anbricht (Biberthaler und Oestern 2012; Müller 2011; Bühren et al. 2016; von Laer et al. 2007).

Der Knochen ist Ursprungs- und Ansatzort für Muskeln. Diese kontrahieren sich auch im Falle einer Fraktur und tragen so, neben dem Trauma selbst, zur Verschiebung der Fragmentstücke bei. Es sind je nach Knochen und je nach genauer Frakturlokalisation, typische Dislokationsmuster zu beobachten. Bei der Femurschaftfraktur kommt es stets zur Verkürzung des betroffenen Beines (Bühren 2004). Röntgenbilder in zwei Ebenen erlauben die Beschreibung der Dislokation(en), es wird dabei immer das distale Fragment beschrieben (Müller 2011; Biberthaler und Oestern 2012; Raschke und Haas 2012). 


\subsubsection{Bruchmuster von Schaftfrakturen: Biegungs-, Quer-, Schräg-, Spiral-, Mehrfragment-, und Trümmerfraktur}

Je nach Größe und Richtung der traumatisch einwirkenden Gewalt auf den Knochen und dem Ausmaß der absorbierten Energie treten unterschiedliche Bruchmuster auf. Im Röntgenbild sind folgende Formen von Schaftfrakturen zu unterscheiden:

Biegungs-, Quer- und Schrägbruch: Bei der Biegungsfraktur kommt es auf der krafteinwirkenden Kortikalisseite zu starker Druckspannung, sodass ein Biegungskeil herausgesprengt wird. Auf der Gegenseite entstehen starke Zugkräfte, sodass der Knochen dort quer einreißt. Ist die Gewalteinwirkung heftig, kurz und schnell, entsteht eine Querfraktur (rechtwinklig zur Längsachse). Verschieben sich die Fragmentstücke nicht, ist dieser Knochenbruch stabil. Eine Sonderform des Querbruchs ist die Schrägfraktur mit einem Bruchwinkel mit mehr als $120^{\circ}$. Da ihre Frakturfläche größer ist als die der Querfraktur, heilt sie schneller aus (Müller 2011; Raschke und Haas 2012, Biberthaler und Oestern 2012; Krischak 2005).

Eine Spiralfraktur wird stets durch indirekt einwirkende Gewalt verursacht - nämlich durch gegenläufige Zugspannungen. So entsteht ein spiralförmiges Bruchmuster, zum Teil mit einem Drehkeil (Müller 2011; Biberthaler und Oestern 2012; Raschke und Haas 2012).

Ist die Gewalteinwirkung sehr stark, treten mehrere Bruchmuster in Kombination auf, und der Knochen zersplittert in multiple Fragmentstücke. Liegen vier bis sechs Fragmente vor, spricht man von einer Mehrfragmentfraktur. Bei mehr als sechs Einzelstücken bezeichnet man den Knochenbruch als Trümmerfraktur. Bei beiden sind die umliegenden Weichteile oft stark verletzt. (Raschke und Haas 2012; Biberthaler und Oestern 2012; Müller 2011). Für die verschiedenen Bruchmuster siehe Abbildung 1.
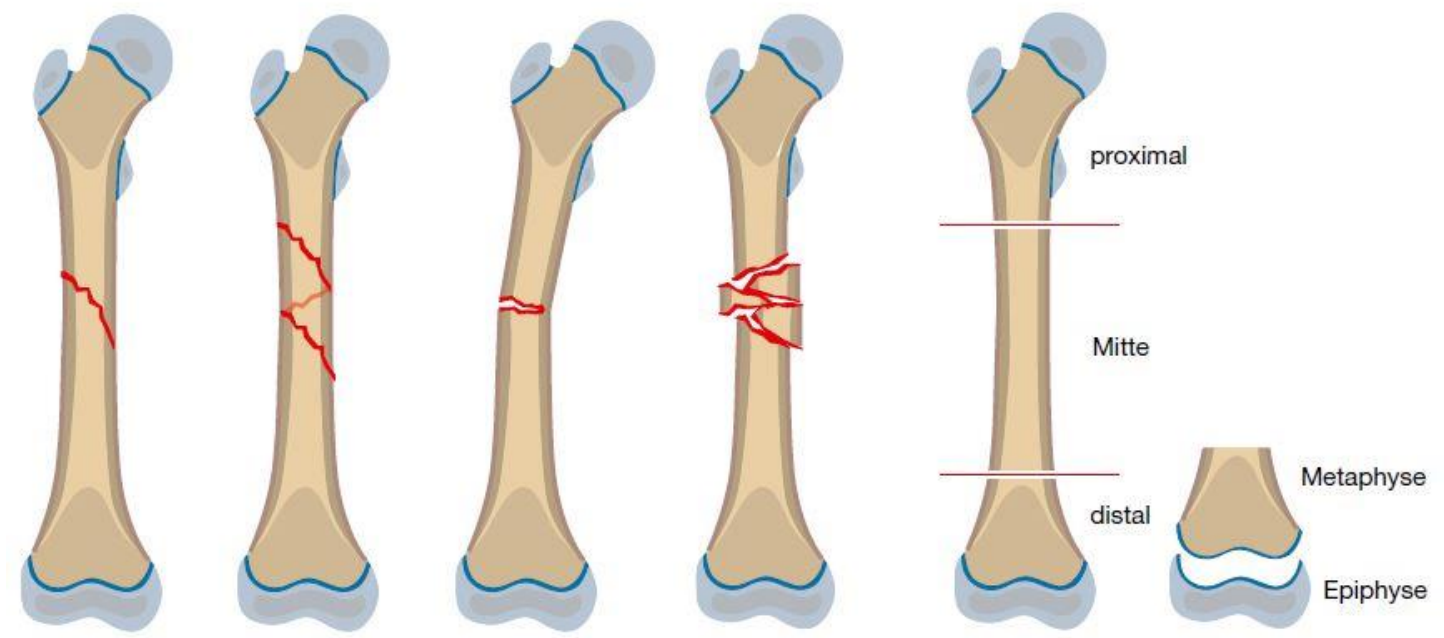

Abbildung 1: Frakturformen (Schrägfraktur, Spiralfraktur, Grünholzfraktur, Trümmerfraktur) sowie Einteilung von Knochenabschnitten 


\subsubsection{Klassifikationen für Frakturen im Wachstumsalter}

Um Kinder trotz der anatomischen Besonderheiten des kindlichen Skeletts (Wachstumsfugen, Korrekturpotenz, rasche Bruchheilung) und trotz der altersspezifischen Verletzungsmuster sicher und adäquat zu behandeln, bedarf es separater Klassifikationen neben den Frakturklassifikationen für Erwachsene.

Die beiden wichtigsten kinderspezifischen Klassifikationen für Frakturen der langen Röhrenknochen sind die Einteilung der Arbeitsgemeinschaft für Osteosynthese (AO) gemäß Slongo und des Vereins „Licht und Lachen für kranke Kinder. Effizienz in der Medizin e.V." (Li-La Klassifikation). Beide Einteilungen zeigen eine gute Reliabilität und sind gleichwertig hinsichtlich ihrer Anwenderfreundlichkeit (Vogel 2012; Weinberg und Schneidmüller 2010a; Kamphaus et al. 2015).

Im Folgenden wird exemplarisch auf die Li-La Klassifikation² eingegangen:

Die Klassifikation sollte „folgende Merkmale aufweisen: kinderspezifisch, exakt vergleichbar, hinreichend differenzierbar, einfach anzuwenden („Narrensicherheit“), sich selbst erklären“ (von Laer et al. 2005).

Ähnlich der AO-Klassifikation für Frakturen im Erwachsenenalter wird ein ZahlenBuchstaben-Code genutzt, um die Fraktur zu beschreiben. Der erste Teil gibt Informationen über den Frakturort an Skelett und Knochen. Im Weiteren wird auf die Morphologie eingegangen und abschließend das Dislokationsausmaß bezeichnet. An sechster Stelle des Codes kann ein Zusatz stehen.

Für die erste Stelle werden die Zahlen eins bis vier für den oder die frakturierten Knochen vergeben:

$$
\begin{aligned}
& \text { 1- Humerus } \\
& \text { 2- Radius/Ulna } \\
& \text { 3- Femur } \\
& \text { 4- Tibia/Fibula }
\end{aligned}
$$

An zweiter Stelle folgt die Angabe zur Lokalisation des Bruchs im Knochen:

1- Proximales Segment (proximale Epi-/Metaphyse)

\footnotetext{
2 Der im Jahr 2000 gegründete gemeinnützige Verein „Licht und Lachen für kranke Kinder. Effizienz in der Medizin e.V.“ (Li-La e.V.) hat sich den Auftrag gegeben, Ärzte in ihrer fachlichen Kompetenz, ihrem pädiatrischenn Wissen und Umgang mit kranken Kindern zu schulen, damit diese eine bessere kindbezogene Behandlung erfahren. Um dieses Vorhaben zu realisieren, führt der Verein multizentrische Studien durch, unterhält ein Online-Dokumentationssystem, veranstaltet Fortbildungen für Ärzte, versteht sich als Anlaufstelle für Eltern und engagiert sich als Herausgeber einer Klassifikation für Frakturen der langen Röhrenknochen (http://www.li-la.org/content/index.php?id=2, Zugriff am 13.07.16).
}

Die Klassifikation wurde von Li-La e.V. in Zusammenarbeit mit dem Institut für Evaluative Forschung in der Orthopädischen Chirurgie der Universität Bern (IEFO) entwickelt und ist bereits evaluiert und weiterentwickelt worden. 
2- Mitte (Diaphyse)

3- Distales Segment (distale Epi-/Metaphyse)

Die peripheren Segmente 1 und 3 werden mit dem „Quadrat über der dazugehörigen Fuge“ definiert („Zirkelschlag von den Ecken der Epiphysenfuge aus“, Li-La e.V.). Ein Unterschied zur pädiatrischen AO-Klassifikation nach Slongo besteht darin, dass hier bei den paarigen Knochen lediglich die Metaphyse von Radius bzw. Tibia ausgemessen wird (siehe Abbildung 2 und Abbildung 3).

An Position drei wird zwischen Gelenk- (Code: a) und Schaftfraktur (Code: s) unterschieden. Diese Angabe ist relevant für Indikationsstellung und Therapie. Im Gegensatz zur pädiatrischen AO-Klassifikation werden Epiphysenlösungen zu den (periphersten) Schaftfrakturen gezählt. Als Gelenkfrakturen gelten nur Frakturen, bei denen die Gelenkfläche verletzt ist. Intraartikuläre Epiphysenfrakturen würden demnach zu den Gelenkfrakturen zählen (Vogel 2012; Weinberg und Schneidmüller 2010a; Kamphaus et al. 2015).

An vierter Stelle wird die Morphologie der Gelenk- und Schaftfrakturen detailliert für jeden Knochen beschrieben. Ihr wird eine Zahl von eins bis fünf zugeordnet. Bei den Schaftfrakturen liegt folgendes Muster von Frakturgruppen zu Grunde: Gruppe 1 umfasst Epiphysenlösungen, Gruppe 2 fasst die „stabilen“ Frakturen zusammen, Gruppe 3 entspricht den Quer-, Schräg- und Spiralfrakturen, Gruppe 4 vereint die Mehrfragmentfrakturen und unter die fünfte Gruppe „andere“ fallen alle übrigen Frakturen (Weinberg und Schneidmüller 2010a; Vogel 2012; von Laer et al. 2007). Für die Gelenkfrakturgruppen siehe Tabelle 1.

Tabelle 1: Gruppen der Schaft- und Gelenkfrakturen nach Weinberg und Schneidmüller (2010a)

\begin{tabular}{|c|c|}
\hline Gruppen der Schaftfrakturen & Gruppen der Gelenkfrakturen \\
\hline $\begin{array}{l}\text { 1: Epiphysenlösungen (Salter I und II) } \\
\text { 2: Meta- bzw. diaphysäre Grünholz- und Wulst- } \\
\text { frakturen („stabile“ Frakturen) } \\
\text { 3: Quer-, Schräg- und Spiralfrakturen } \\
\text { 4: Mehrfragmentfrakturen } \\
\text { 5: andere }\end{array}$ & $\begin{array}{l}\text { 1: epiphysäre Frakturen bei offenen Fugen } \\
\text { (Salter-Harris-III-Frakturen) } \\
\text { 2: epimetaphysäre Frakturen bei offenen Fugen } \\
\text { (Salter-Harris-IV-Frakturen) } \\
\text { 3: Two-plane-Übergangsfrakturen } \\
\text { 4: Tri-plane-Übergangsfrakturen } \\
\text { 5: andere } \\
\text { Ausnahme: distaler Humerus (seperate } \\
\text { Einteilung) }\end{array}$ \\
\hline
\end{tabular}


Die fünfte Stelle wird mit der (subjektiven) Beschreibung des Dislokationsausmaßes belegt:

0- undisloziert

1- tolerabel disloziert,

2- nicht tolerabel disloziert

Von Laer et al. (2005) befanden Abweichungen von $10-25^{\circ}$ als tolerabel disloziert und 25-30 als nicht mehr tolerable Achsabweichungen.

Falls isoliert nur Elle oder Wadenbein frakturiert sind, wird an sechster Stelle ein U (=Ulna) oder F (=Fibula) eingefügt, andernfalls entfällt sie. (Vogel 2012; Weinberg und Schneidmüller 2010a; Kamphaus et al. 2015). Für eine Übersicht der Schaftfrakturen siehe Abbildung 4.

Kamphaus et al. (2015) erforschten die Intra- und Interobserverreliabilität der Li-La Klassifikation. Sie fanden heraus, dass diese zuverlässiger (reliabler) ist im Vergleich zu vielen anderen etablierten frakturspezifischen Klassifikationen (bspw. Klassifikation für proximale Humerusfrakturen nach Neer). Des Weiteren stehe sie der PCCF in der Anwendbarkeit in nichts nach: „Somit weist die Li-La Klassifikation eine vergleichbare Reliabilität zur AO-Klassifikation für Frakturen im Wachstumsalter auf." (Kamphaus et al. 2015) 


\section{LiLa Klassifikation}

Version 2

\begin{tabular}{|c|c|c|c|c|c|}
\hline $\begin{array}{l}\text { 1. Stelle } \\
\text { Lokalisation } \\
\text { im Skelett: } \\
(1-4)\end{array}$ & $\begin{array}{l}\text { 2. Stelle } \\
\text { Lokalisation } \\
\text { im Knochen } \\
\text { (Segment): } \\
(1-3)\end{array}$ & $\begin{array}{l}\text { 3. Stelle } \\
\text { Morphologie: } \\
\text { - Gelenk (a) } \\
\text { - Schaft (s) }\end{array}$ & $\begin{array}{l}\text { 4. Stelle } \\
\text { Spezifizierungen } \\
\text { Morphologie: } \\
\text { - Gelenk (1-5) } \\
\text { - Schaft (1-5) }\end{array}$ & $\begin{array}{l}\text { 5. Stelle } \\
\text { Dislokationsausmass: } \\
\text { - undisloziert }(0) \\
\text { - tolerabel }(1) \\
\text { - nicht tolerabel (2) }\end{array}$ & $\begin{array}{l}\text { 6. Stelle } \\
\text { (Ausnahme) } \\
\text { paariger Knochen } \\
\text { nicht tragend } \\
\text { - Ulna }(\boldsymbol{U}) \\
\text { - Fibula }(\boldsymbol{F})\end{array}$ \\
\hline
\end{tabular}

- bei paarigen Knochen wird jeweils der haupttragende Knochen klassifiziert: Radius oder Tibia

- soll der Gegenknochen klassifiziert werden, so wird an 6. Stelle das U bzw. das F eingefügt (siehe Gelenkfrakturen Olekranon)

- die Metaphyse wird mit dem Quadrat über der zugehörigen Fuge definiert (Zirkelschlag von den Ecken der Epiphysenfuge aus)

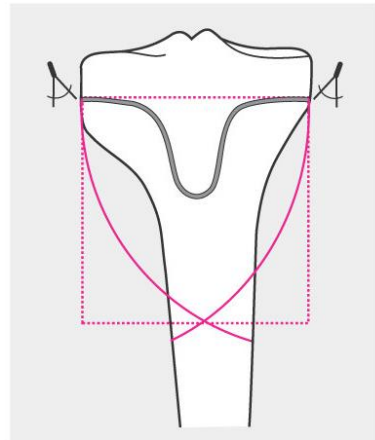

Gelenkverletzungen die statistisch nicht ins Gewicht fallen, werden an der jeweiligen Lokalisation mit 5= andere klassifiziert (proximaler Humerus, proximaler und distaler Radius, proximale und distale UIna und proximales Femur)

Abbildung 2: Übersicht Li-La Klassifikation Version 2 und Ermittlung der Epiphysenfuge

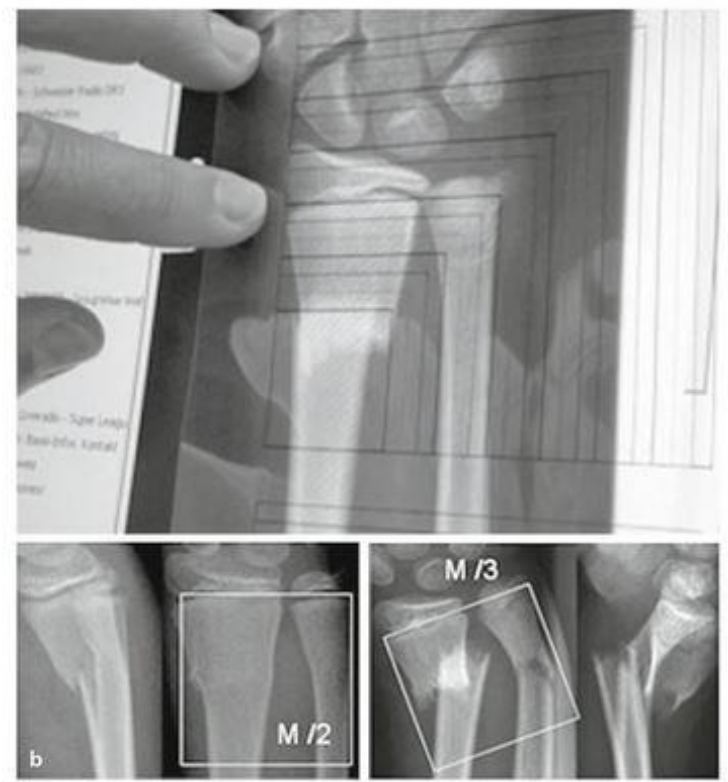

Abbildung 3: Vorgedruckte Quadrate auf einer transparenten Folie, die über die Röntgenaufnahme gelegt werden können, unterstützen die korrekte Identifikation der Metaphyse (Slongo et al. 2010). 


\section{Schaft \#}

1.1-3.s.1-5.0-2.

1. Epiphysenlösung ohne und mit metaphysärem Keil (Salter I und II)

2. metaphysäre Stauchungs- und metaphysäre Grünholz Fx/ diaphysäre Grünholzfrakturen

3. Quer-, Schräg- und Torsions Fx

4. Mehrfragment Fx

5. andere

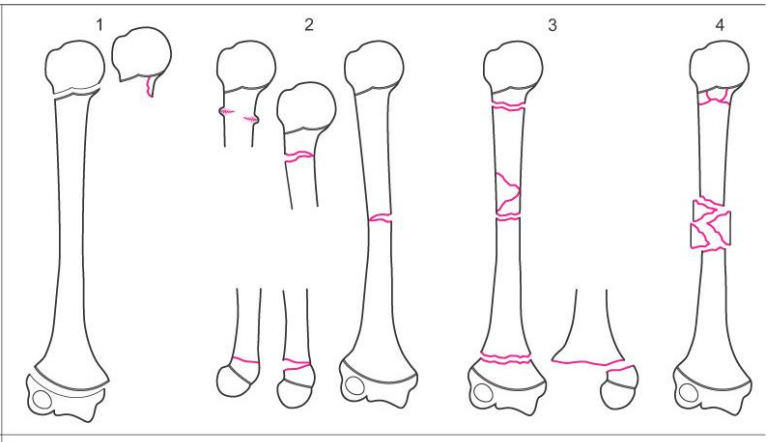

2.1-3.s.1-5.0-2.

1. Epiphysenlösung ohne und mit metaphysärem Keil (Salter I und II)

2. metaphysäre Stauchungs- und metaphysäre Grünholz Fx/ diaphysäre Grünholzfrakturen

3. Quer-, Schräg- und Torsions Fx

4. Mehrfragment Fx

5. andere

3.1-3.s.1-5.0-2.

1. Epiphysenlösung ohne und mit metaphysärem Keil (Salter I und II)

2. metaphysäre Stauchungs- und metaphysäre Grünholz Fx/ diaphysäre Grünholzfrakturen

3. Quer-, Schräg- und Torsions Fx

4. Mehrfragment Fx

5. andere

4.1-3.s.1-5.0-2.

1. Epiphysenlösung ohne und mit metaphysärem Keil (Salter I und II)

2. metaphysäre Stauchungs- und metaphysäre Grünholz Fx/ diaphysäre Grünholzfrakturen

3. Quer-, Schräg- und Torsions Fx

4. Mehrfragment Fx

5. andere
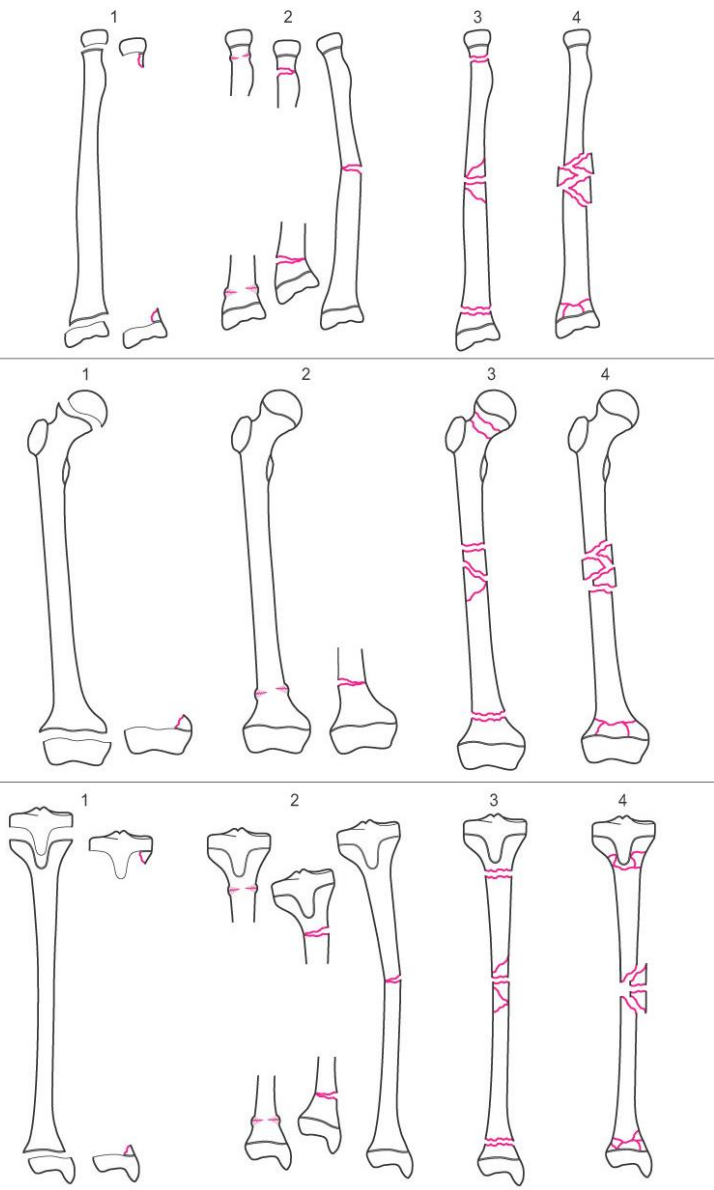

Abbildung 4: Einteilung der Schaftfrakturen der Li-La Klassifikation

\subsection{Behandlungsmaßnahmen}

„Oberschenkelfrakturen im Wachstumsalter heilen immer - unabhängig von der Behandlungsmethode! Die Qualität der Behandlung kann also nicht an der Heilung per se überprüft werden, sondern an der Umsetzung der Komfortwünsche des Patienten: an der frühzeitigen Mobilität und Belastungsmöglichkeit - noch weit vor Konsolidation der Fraktur!" (von Laer et al. 2007) 
Bei der Indikationsstellung für eine Behandlung muss nicht nur auf das Alter des Kindes, die Bruchform, weitere Verletzungen und Grunderkrankungen geachtet werden, sondern es müssen auch Aufwand und Nutzen für die Erkrankten und ihre Eltern abgewogen werden. Es sind die Risiken und mögliche Folge(erkrankunge)n einer Therapie zu bedenken und nicht zuletzt die Kosten und Nutzen abzugleichen. Auch die familiäre Situation des Kindes sollte bei der Indikationsstellung mit einfließen (Worel und Slongo 2010).

Eine optimale Behandlung verbindet beste Heilungschance mit möglichst geringen Komplikationsrisiko (Zimmermann 2008). Gerade bei Kindern sind eine primäre definitive Reposition und Versorgung der Fraktur von großer Bedeutung, da es bei Nachrepositionen und Verfahrenswechsel (und somit möglicher Manipulation der Wachstumsfuge) häufig zu Komplikationen kommt, wie z.B. Beinlängendifferenzen, verzögerter Frakturheilung und Wachstumsstörungen (Zimmermann 2008; von Laer et al. 2007; Worel und Slongo 2010). Von Laer et al. (2007) betonen, dass das Ausheilen der kindlichen Fraktur fast garantiert ist und das „ärztliche Eingreifen“ diesen Prozess nicht gefährden darf. Unabhängig davon, ob eine konservative oder operative Therapie erfolgt, sollten folgende Behandlungsziele erreicht werden:

- schnelle Schmerzlinderung und ein schmerzfreier Verlauf

- schnelle Wiedererlangung der Mobilität (kurze Liegedauer, kurzer Krankenhausaufenthalt)

- frühe Wiederherstellung der Stabilität und Funktionalität (exakte anatomische Reposition und rasche Belastungs- und Bewegungsfähigkeit möglichst vor Frakturkonsolidierung)

- Effizienz (geringer Aufwand sollte bestmögliches Ergebnis bringen)

- komplikationsloser Heilungsverlauf, besonders iatrogen hervorgerufene Wachstumsstörungen müssen verhindert werden (einmalig definitive Versorgung)

- gute Spätergebnisse (minimale Narbenbildung, geringe psychische Belastung für das Kind, vollständige und anatomisch korrekte Ausheilung). (von Laer et al. 2007; Zimmermann 2008; Worel und Slongo 2010)

Es stehen verschiedene Behandlungsmaßnahmen zur Wahl. Dazu zählen die konservativen mit Gipslonguette, Becken-Bein-Gips und Extension sowie die operativen mit extra- und intramedullären Kraftträgern (ESIN, Fixateur externe, Platte, Schraube, Marknagel). Von (Laer 2007) weist darauf hin, dass die operativen und konservativen Behandlungen auch $\mathrm{zu}$ kombinieren sind und nicht unbedingt in Konkurrenz zueinander stehen. Ein weiteres „Heilmittel“ ist die spezifische Fähigkeit des kindlichen Knochens, Achsabweichungen spontan, ohne Eingriff von außen, im Laufe seines Wachstums korrigieren zu können. In der Remodellingphase können durch das periendostale System sowie das asymmetrische Wachsen von 
Epiphysenfugen Achsabweichungen in allen drei Ebenen des Raumes ausgeglichen werden (von Laer 2010; von Laer et al. 2013; von Laer et al. 2007)(vgl. 1.6.1).

\subsubsection{Konservative Therapie der Femurschaftfraktur}

Die nicht invasive Behandlung war bis vor circa 30 Jahren die Therapie der Wahl für kindliche Femurschaftfrakturen. Hier wird durch Ruhigstellung des frakturierten Knochens rasch der Schmerz reduziert und intolerabler Achsfehlstellung vorgebeugt bzw. diese wird behoben (Redression). Nach von Laer et al. (2007) gilt dies weiterhin als präferierte Therapie altersunabhängig für die proximalen undislozierten Femurschaftfrakturen sowie für die raren völlig undislozierten diaphysären Schaftfrakturen. Auch für Kinder unter vier Jahren ist die konservative die Therapie erster Wahl, da eine Allgemeinnarkose nicht indiziert sei.

Bei der konservativen Therapie entfallen die Risiken eines invasiven Eingriffs, jedoch leidet der Patient unter mehrwöchiger Immobilisation, womöglich langer Hospitalisierung, ist wiederholt der Röntgenstrahlung ausgesetzt. Eine inexakte und nicht fachkundig angelegte konservative Therapie führt zu sonst vermeidbaren Komplikationen (sekundäre Dislokationen, Druckulzera, Kontrakturen, Schmerzen, bleibende Fehlstellung) (Kraus 2007; Worel und Slongo 2010). Wenn nachreponiert werden muss, bleibt eine ausreichende Fixation meist aus und die Gefahr für Komplikationen, z.B. Overgrowth, erhöht sich (Zimmermann 2008).

\subsubsection{Pavlik-Bandage, Becken-Bein-Gips, Gips(longuette)}

Im Neugeborenenalter kann eine Pavlik-Bandage angelegt werden (siehe Abbildung 5). Kleinkinder werden mit dem Becken-Bein-Gips versorgt und für größere Kinder eignet sich die Retention mit einer Gipslonguette (siehe Abbildung 6). Abbildung 7 zeigt Röntgenbilder einer siebenjährigen Patientin, die mit einem Becken-Bein-Gips behandelt wurde.

Falls unmittelbar vor der Anlage die Fraktur reponiert worden war, ist mit einer Schwellung zu rechnen und der Gips muss, falls er im Rahmen einer primär geschlossenen Reposition zirkulär angelegt worden ist, bis auf die Haut längs gespalten werden. Sobald die Schwellung zurückgegangen ist (nach circa vier Tagen), kann ein geschlossener Gipsverband angelegt werden. Zur Anlage eines Gips- oder Kunststoffverbandes ist keine Narkose notwendig, möglicherweise aber eine Sedation (von Laer et al. 2007; Dietz 2011; Biberthaler und Oestern 2012).

Der Gips sollte bis zur Konsolidierung der Fraktur getragen werden, die Zeitspanne kann je nach Alter zum Unfallzeitpunkt und je Frakturlokalisation variieren. Laut Marzi 
(2010) sollten diaphysäre Frakturen bei Kindern unter fünf Jahren für ein bis drei Wochen geschont werden, Patient(inn)en im Alter zwischen fünf und zehn Jahren benötigen vier bis fünf Wochen bis zur Bewegungsstabilität und über zehn Jahre alte Kinder sollten das Femur für vier bis sechs Wochen ruhig gestellt bekommen (Worel und Slongo 2010; Weinberg und Schneidmüller 2010b).

Nachteile der Therapie mit einem Gipsverband sind die eingeschränkte Bewegungsfreiheit, die daraus resultierende Muskelatrophie und, besonders bei einem Becken-BeinGips, der pflegerische Aufwand für die Eltern. Wird der Gips fehlerhaft angelegt, kann es zu Druckulzera, Nervenschäden, Kompartmentsyndrom und als Spätfolge zu einer Achsfehlstellung und/oder Beinlängendifferenz kommen (Kraus 2007).

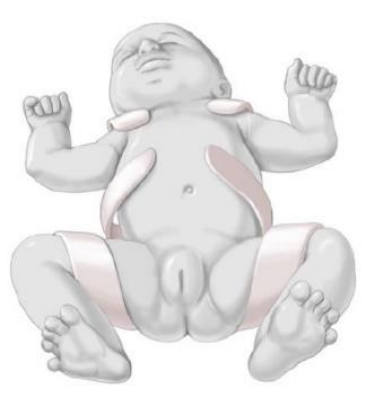

Abbildung 5: Pavlik-Bandage

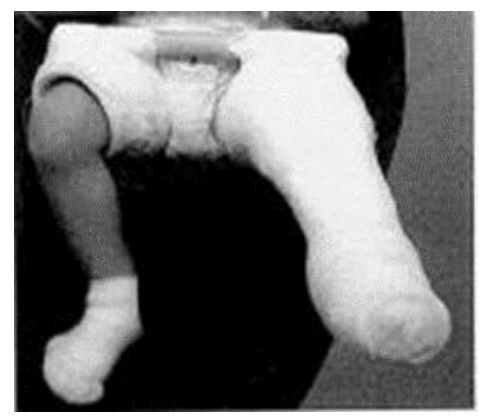

Abbildung 6: Becken-Bein-Gips
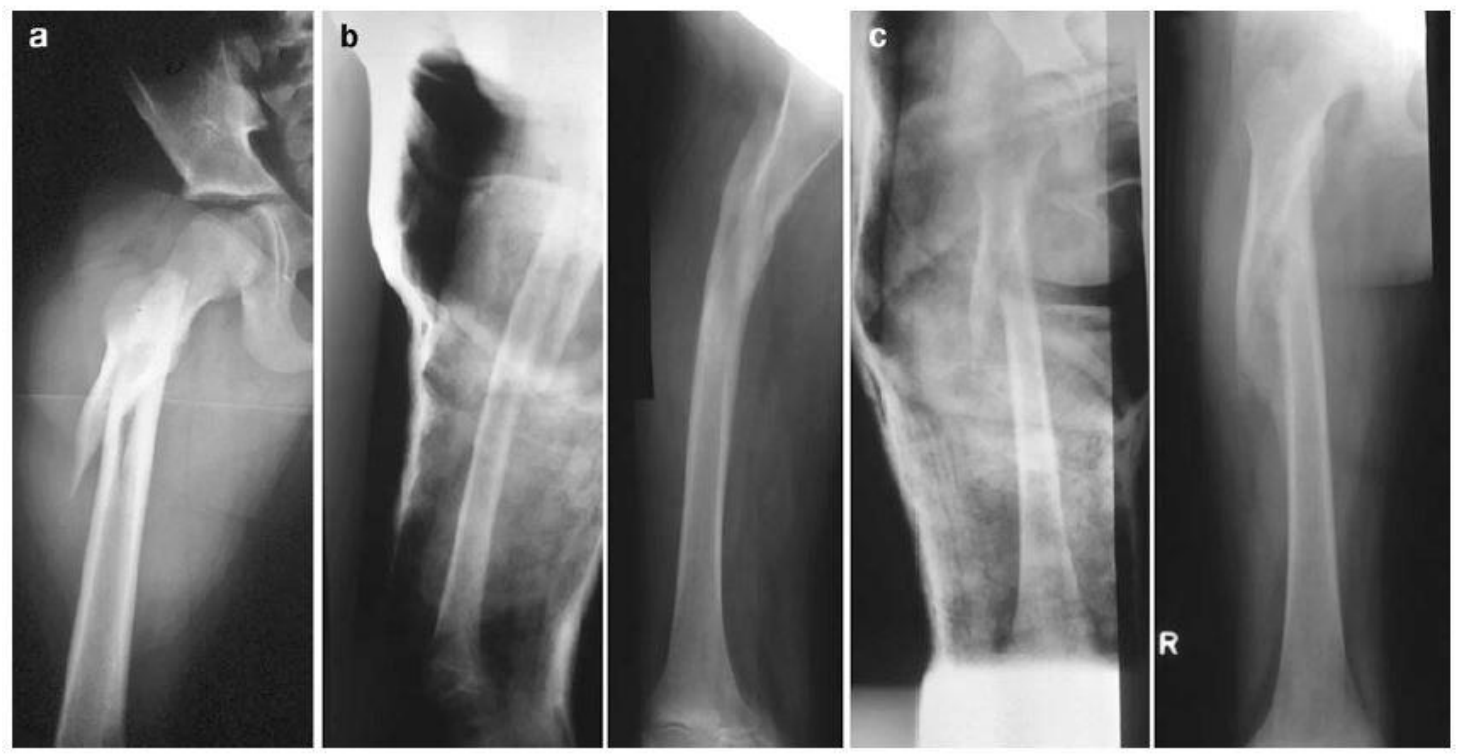

Abbildung 7: Proximale Femurschaftfraktur bei einem siebenjährigen Mädchen, a) Unfallbild, b) nach Anlage eines Becken-Bein-Gips', c) Ausheilung nach zwei Monaten 


\subsubsection{Extensionsbehandlung}

Diese Behandlung nutzt das Körpergewicht des Patienten als Gegenzug, um die Muskelkräfte, die auf die Fraktur wirken, zu neutralisieren und so dem Knochen die Retention in korrekter anatomischer Achsenstellung zu ermöglichen.

Die Overhead-Extension ist bei Kindern bis zu zwei Jahren indiziert. Das Kleinkind liegt auf dem Rücken, die Hüfte ist rechtwinklig gebeugt und die Kniegelenke gestreckt. Über Heftpflasterverbände vom Fuß bis zum proximalen Oberschenkel wird Dauerzug mit Gewichten angelegt, sodass das Gesäß leicht von der Unterlage abhebt (siehe Abbildung 8 und 9). Abbildung 10 zeigt Röntgenaufnahmen eines vier Monate alten Säugling, der für zehn Tage mit einer Overhead-Extension behandelt wurde.

Für Kinder im Alter von drei bis zehn Jahren, bei denen eine Extensionsbehandlung indiziert ist (z.B. in ressourcenarmen Ländern), werden bei rechtwinkliger Position von Hüfte und Kniegelenk Drähte in den suprakondylären Oberschenkelknochen eingebracht. Über einen Extensionsbügel werden Gewichte angehängt (Zimmermann 2008; Biberthaler und Oestern 2012; Worel und Slongo 2010; von Laer et al. 2007).
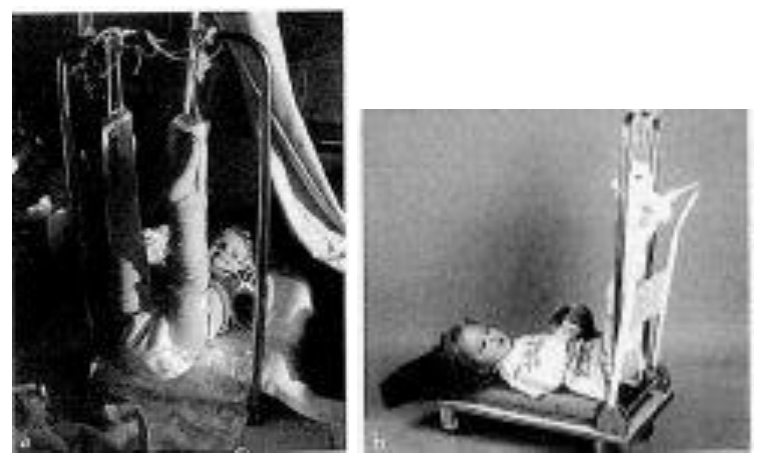

Abbildungen 8 und 9 : Kinder in Behandlung mit Overhead-Extension

Diese Behandlung weist eine Vielzahl von Nachteilen auf. Es kommt zur Immobilisation der Patient(inn)en mit all den bekannten negativen Konsequenzen. Falls es keine Möglichkeit zur Heimextension gibt, müssen die Kinder über mehrere Wochen hospitalisiert werden. Neben einem erheblichen Aufwand für das Pflegepersonal stellt dies eine große psychische Belastung für Kind und Eltern dar. Bei der Heftpflasterextension kann es zu Hautarrosionen kommen, bei der Extensionsbehandlung mit suprakondylären Nägeln zu Pin-Tract-Infektionen, bei beiden zu Lagerungsschäden und Kompartmentsyndrom des Unterschenkels (Böhm 2007; Zimmermann 2008; von Laer et al. 2007; Biberthaler und Oestern 2012). In der Literatur werden weitere Probleme bezüglich der Frakturheilung beschrieben: Es kommt zu Achsenfehlern, verzögerter Konsolidierung, Nachrepositionen und somit vermehrter Belastung durch Röntgenstrahlung. 
Insgesamt weist die Komplikationsrate je nach Quelle erhebliche Unterschiede auf. Von Laer et al. (2007) beispielsweise beschreiben Beinlängendifferenzen in $70 \%$ der Fälle nach einer Extensionstherapie und somit doppelt so häufig wie bei anderen Therapien.

Die Diskussion zur Extensionstherapie als zeitgemäßes Mittel wird sehr kontrovers geführt: Von Laer et al. (2007) bezeichnen diese Therapie als „unwürdige Behandlungsmethode, die heute weder einem Erwachsenen noch einem Kind zugemutet werden darf".

Weinberg und Schneidmüller (2010b) empfehlen eine konservative Therapie für unter Dreijährige bei Patient(inn)en, die weniger als $15 \mathrm{~kg}$ wiegen und bei einfachen Schrägund Torsionsfrakturen. Eine Overheadextension sollte nur im Falle einer „stärkeren Verkürzung oder Achsfehlstellung“ (Weinberg und Schneidmüller 2010b) erfolgen und diese nach maximal zwei Wochen von der Therapie mit Becken-Bein-Gips abgelöst werden. Ist die Fraktur tolerabel disloziert, so wird sie von Beginn an vier Wochen lang mit dem Becken-Bein-Gips therapiert.

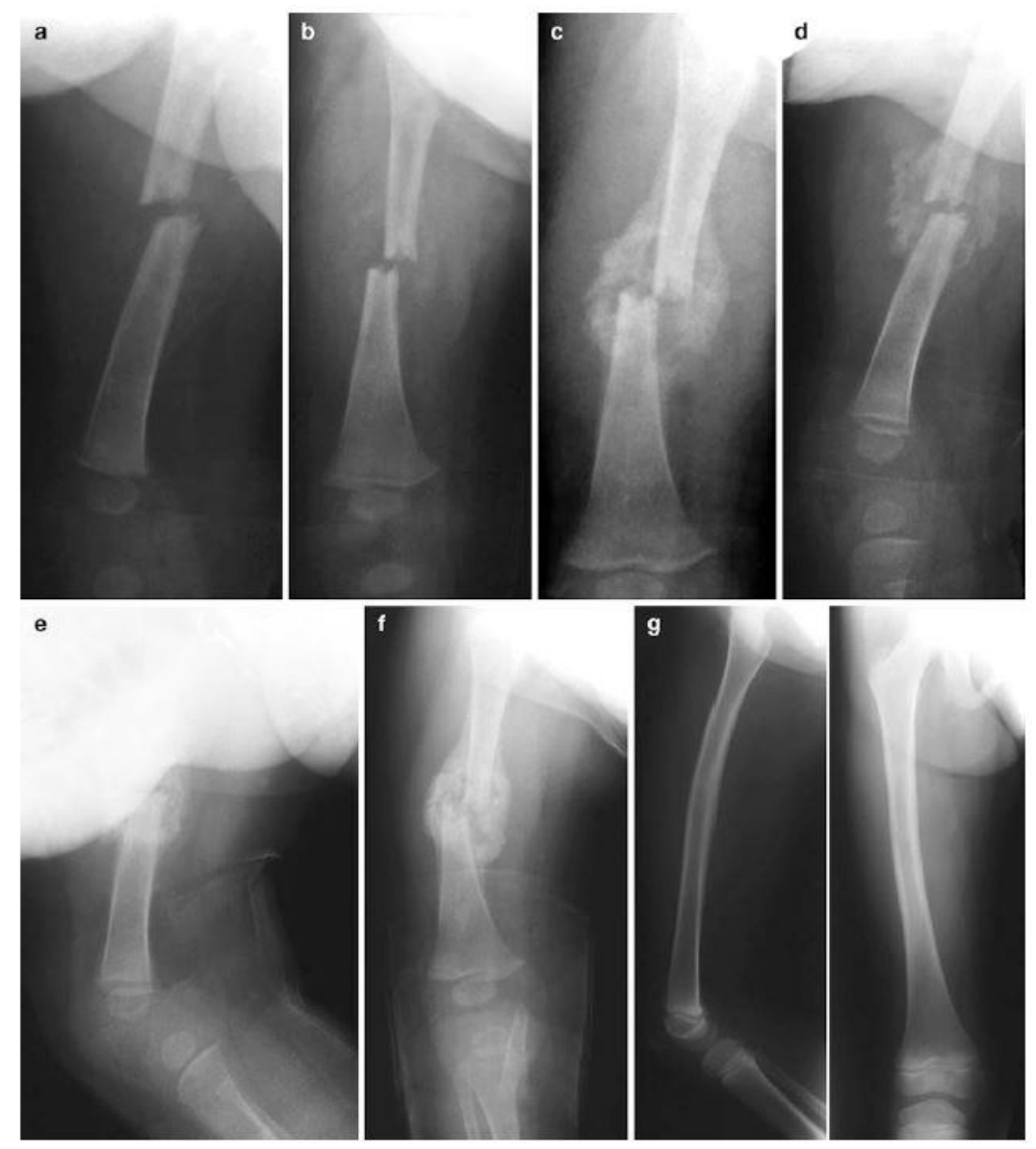

Abbildung 10: Querfraktur eines vier Monate alten Säuglings

a-b) Unfallbild, c-d) nach zehntägiger Extentionstherapie, e-f) Therapieverlauf nach einem Monat, g) Kontrolluntersuchung nach vier Jahren 


\subsubsection{Operative Therapie der kindlichen Femurschaftfraktur}

Für die operative Therapie bei Kindern stehen folgende Implantate zur Verfügung: intramedulläre (Marknagel, ESIN, Kirschner-Drähte, Schrauben) und extramedulläre Kraftträger (Platte, Fixateur externe).

Die Deutsche Gesellschaft für Kinderchirurgie empfiehlt in der S1-Leitlinie für Femurschaftfrakturen im Kindesalter (publiziert bei AWMF online, Stand 09/2014) die operative Versorgung ab dem dritten Lebensjahr. Es wird die Verwendung eines Fixateurs externe bei offenen, sehr instabilen Trümmerfrakturen oder im Falle eines Polytraumas empfohlen. Alle anderen Frakturen sind mit der elastisch stabilen intramedullären Nagelung zu richten, bei größeren/schwereren (>50 kg) Kindern kann ein Adoleszentennagel verwendet werden (http://www.awmf.org/leitlinien/detail/II/006016.html).

Buechsenschuetz et al. (2002) stellten die konservative Behandlung mit Dauerzug (Traktion) oder Gips der operativen ESIN-Behandlung gegenüber und untersuchten Therapieergebnisse, Zufriedenheit der Eltern und Kosten bei einem Kollektiv mit 68 Patient(inn)en. Bei den beiden Behandlungsmethoden unterschieden sich lediglich die Kosten (die konservative Therapie ist kostenungünstiger) und die Akzeptanz der Eltern (mit der ESIN-Therapie waren die Eltern zufriedener). Jedoch wurden mit beiden gleichwertig gute Ergebnisse erzielt. Aus Gründen wie diesen ist die operative Therapie - vor allem mit elastischen Federnägeln - die inzwischen am häufigsten angewandte Therapie bei kindlichen Femurschaftfrakturen, auch bei den unter Dreijährigen (Strohm und Schmittenbecher 2015).

Ramseier et al. (2010) verglichen operative Therapien (Platte, ESIN, Fixateur externe und Marknagel) untereinander im Hinblick auf die Komplikationsrate und die Behandlungsergebnisse. Signifikant mehr Komplikationen erlitten Kinder, die mit dem Fixateur externe behandelt worden waren. Insgesamt hatten alle Therapiemethoden unterschiedlich spezifische Komplikationen, sodass die Autoren keine Therapie generell empfehlen. Sie weisen darauf hin, dass die beste Therapie für den jeweiligen Patienten von der Frakturcharakteristik, Begleitverletzungen, Alter und Körperbau des Patienten oder der Patientin, den familiären Gegebenheiten und auch der Expertise des Operateurs abhängt.

Im Folgenden werden oben erwähnte operative Behandlungsmethoden vorgestellt mit ihren Besonderheiten, der Indikation, möglichen Komplikationen sowie ihrer Rezeption in der Literatur. 


\subsubsection{ESIN}

Bei der elastisch stabilen intramedullären Nagelung wird die Fraktur durch die Aufspannung von zwei gebogenen (Titan-)Drähten im Markraum stabilisiert. Sie ist mittlerweile die häufigste Behandlungsmaßnahme bei Kindern im Alter von drei bis 15 Jahren, da sie eine „minimal invasive, minimal traumatisierende, bewegungs- und teilbelastungsstabile, biologische und kinderfreundliche Osteosynthese" darstellt (AO Teaching Video 2002). Abbildung 11 zeigt die wesentlichen Schritte der ESINImplantation:
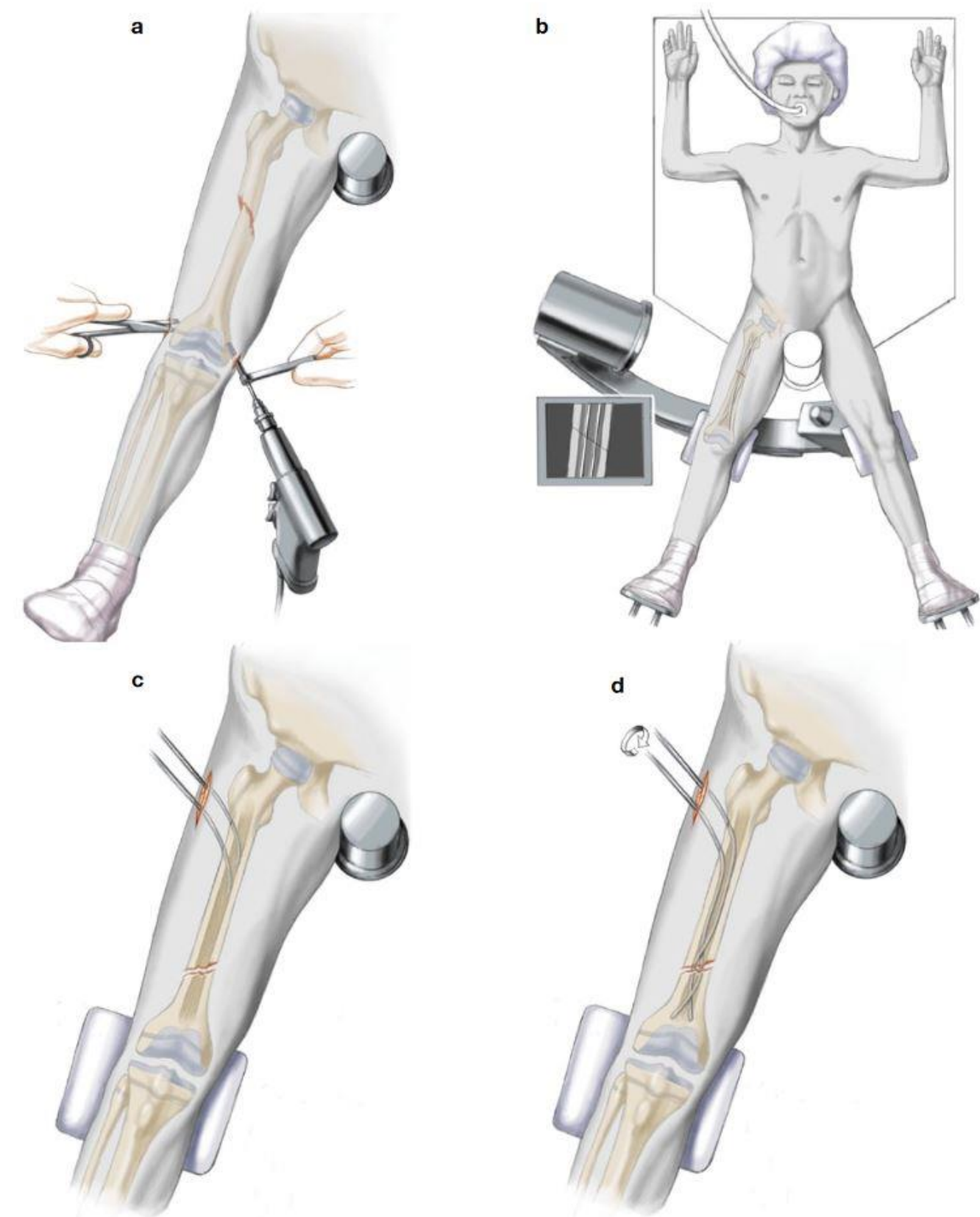

Abbildung 11: Retro- und anterogerade Nagelung einer Querfraktur mit elastisch stabilen Federnägeln und Endcaps.

Je nach Lokalisation der Fraktur werden die Drähte aszendierend retrograd (Femurschaft und proximales Schaftdrittel, siehe a) oder deszendierend anterograd (distales Schaftdrittel und distale Metaphyse, siehe c-d) eingebracht. Die korrekte Nageldicke entspricht 30-40\% des Markraums. Vor dem 
Einführen in den Knochen werden die Nägel auf den dreifachen Durchmesser vorgebogen. Je stärker vorgebogen wird, desto höher ist der innere Anpressdruck (Worel und Slongo 2010). Die Länge sollte der des Oberschenkelknochens entsprechen. Dicke und Länge der Nägel müssen identisch sein.

Die Nägel werden circa $2 \mathrm{~cm}$ über dem Kortikalisniveau gekürzt. Wird der Draht unzureichend gekürzt, kann er die Haut durchstechen und diese sich infizieren. Wird eine komplexe Fraktur, z.B. eine längere Spiralfraktur, mit der ESIN-Methode behandelt und die Stabilität bleibt aus, so können Endkappen hilfreich sein. Diese werden über die Drahtenden gestülpt und in die Kortikalis eingedreht. Einige Autoren empfehlen das Aufsetzen von Plastikkappen auf die überstehenden Drahtenden, um die Weichteile zu schützen.

Post-operativ ist keine weitere Ruhigstellung notwendig. Es kann sofort teilbelastet werden. Nach vier bis sechs Wochen sollten röntgenologische Stellungs- und Konsolidierungskontrollen durchgeführt werden und zumeist kann vollbelastet werden. Acht Wochen nach der Frakturkonsolidierung können die Kinder wieder Sport betreiben. Einige Autoren berichten darüber, dass einfache Querfrakturen schon vor den vier bis sechs Wochen vollbelastet werden können (Marzi 2011). Abbildung 13 zeigt Röntgenaufnahmen eines Patienten bzw. einer Patientin mit distaler Querfraktur, bei dem bzw. der die ESIN-Technik angewandt wurde.

In der Literatur wird über folgende Komplikationen berichtet:

- Wundheilungsstörungen oder Knocheninfektionen

- Intolerable Achsabweichungen des Femurs

- Beinlängendifferenzen, Wachstumsstörungen

- Instabilität, Teleskoping, sekundäre Dislokation

- Intraoperativ: Korkenzieher-Phänomen, Kortikalisperforation und Aussprengung eines dritten Fragments,

- Pseudobursa, Serom, Hämatom, Hautperforation durch überstehende Nagelenden

- Passagere Bewegungseinschränkung der Flexion im Knie

- Kompartmentsyndrom

- Implantatlockerung, -dislokation, -bruch

- Schmerzen durch Weichteilirritation

- Refraktur

Die Komplikationsrate der ESIN-Therapie wird in der Literatur mit $6 \%$ bis zu $50 \%$ sehr unterschiedlich angegeben. Auch wenn Komplikationen eintreten, sind die Langzeitergebnisse hingegen exzellent bis zufriedenstellend und die Methode ist das am häufigsten eingesetzte operative Verfahren zur Frakturstabilisierung. Leitlinien von AWMF, DGU und AAOS empfehlen die Therapie erst ab einem Patient(inn)enalter von drei bzw. vier Jahren, sie wird jedoch im Alltag in Deutschland auch bei unter Dreijährigen in über 50\% der Fällen angewendet (Strohm und Schmittenbecher 2015).

Um Komplikationen bei der ESIN-Therapie vorzubeugen, wird wiederholt auf die notwendige Genauigkeit in der Operationstechnik hingewiesen. Im Hinblick auf die Verfeinerung der Technik wurden Studien durchgeführt. So überprüften z.B. Kaiser et al. (2012) in einer In-vitro-Studie den Einfluss des Vorbiegens der Federnägel auf die Sta- 
bilität bei Spiralfrakturen. Es zeigte sich, dass beim Vorbiegen um $60^{\circ}$ die Steifheit der Frakturzone signifikant erhöht werden konnte im Vergleich zu ungebogenen Nägeln. Bei $30^{\circ}$ hingegen konnte keine signifikante Stabilitätserhöhung festgestellt werden. Kaiser et al. (2012) empfehlen deshalb das Vorbiegen um mehr als $30^{\circ}$, um die Stabilität bei der Nagelung zu erhöhen und Komplikationen zu vermeiden.

Eine weitere Modifikationsmöglichkeit, um die Stabilität bei der Nagelung mit Federnägeln zu erhöhen, scheint das Einbringen eines dritten Nagels zu sein (siehe Abbildung 14). Kaiser et al. (2014) veröffentlichten Ergebnisse eines In-vitro-Modells (Spiralfraktur) und einer klinischen Studie mit 18 Kindern. Bei der biomechanischen Testung des Modells zeigte sich eine signifikant höhere Versteifung des Knochens und eine bessere Ausrichtung der Fragmente, wenn ein dritter, zusätzlicher Nagel von antero-lateral eingeführt worden war. Die Autoren sahen bei allen 16 Patient(inn)en, die mit drei Nägeln behandelt wurden (dritter Nagel eingeführt von antero-lateral oder antero-medial, je nach Frakturtyp) sehr gute Ergebnisse in den Follow-Up Untersuchungen. Es musste keiner der Patienten und keine der Patientinnen re-operiert oder ihre Frakturen zusätzlich stabilisiert werden.

Die Deutsche Gesellschaft für Kinderchirurgie teilt die Empfehlungen für eine Therapie anhand des Patient(inn)enalters ein. Jedoch sollte auch das Gewicht berücksichtigt werden. Moroz et al. (2006) zeigten in einer Studie mit 229 Kindern, dass das Komplikationsrisiko um ein Fünffaches höher war für Kinder, die mehr als 49 kg wogen. Bei schweren oder größeren Kindern sollte deshalb auch an eine alternative Marknagelung mit dem Adoleszentennagel (ALFN) gedacht werden. 


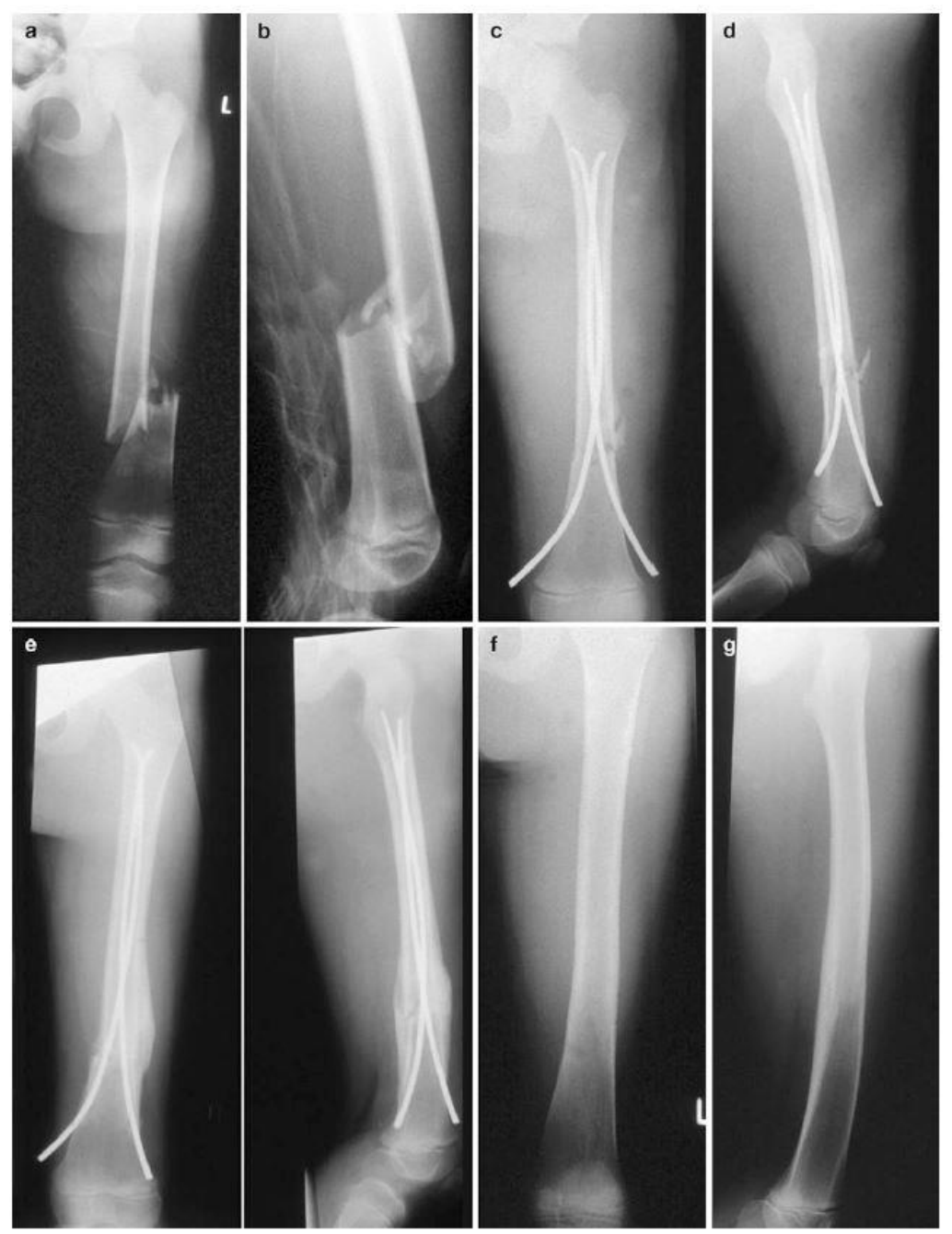

Abbildung 12: Distale Querfraktur, a-b) Röntgenbilder nach dem Unfall, c-d) Versorgung mit ESIN, e) Konsolidierung vor der Metallentfernung, f-g) Ausheilung - Röntgenaufnahme ein Jahr nach dem Frakturereignis

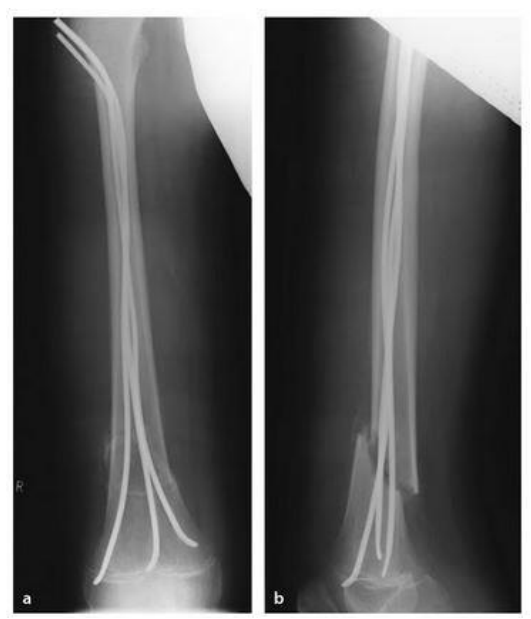

Abbildung 13: Eine distale Femurschaftfraktur, die bei unzureichender Stabilität mit drei elastisch stabilen Federnägeln versorgt wurde. 


\subsubsection{Platte}

Die Plattenosteosynthese wird heute seltener angewandt, meist als Alternative bei adoleszenten und/oder adipösen Patient(inn)en sowie vorzugsweise bei langer Spiralschaft- oder Trümmerfraktur (Worel und Slongo 2010).

Die Platte wird als extramedullärer Kraftträger auf dem Periost angebracht und mit Schrauben befestigt, sodass beide Frakturenden möglichst spaltlos miteinander in Kontakt stehen und komprimiert werden. Stehen die Bruchstücke direkt aufeinander, kann die primäre Frakturheilung ablaufen (Kontaktheilung). An den übrigen Stellen, an denen ein Spalt bleibt, wird der Knochen neu gebildet (sekundäre Frakturheilung mit Kallusbildung/Spaltheilung) (Raschke und Haas 2012). Siehe Abbildung 15.

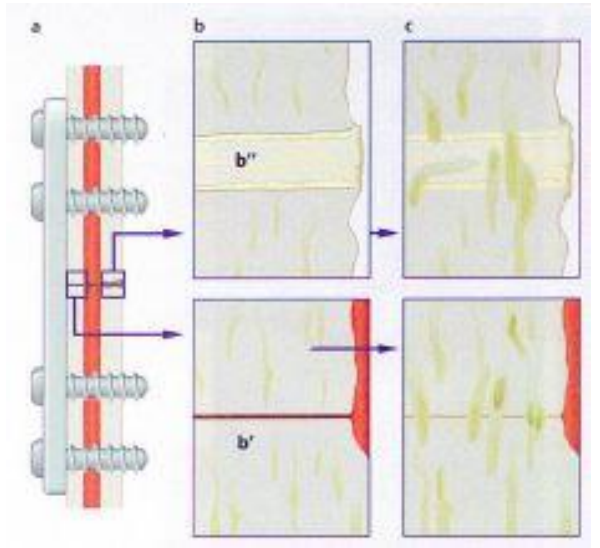

Abbildung 14: Spalt (b")- und Kontaktheilung (b')

Ziel dieser Behandlung ist es, die Frakturzone zu überbrücken und dem Knochen in korrekter Rotations-, Achs- und Längenstellung die Chance zu geben, die Frakturzone zu organisieren und auszuheilen (Marzi 2011).

Zur Periostschonung wird die "Limited Contact Dynamic Compression Plate" (LCDCP) empfohlen, bei der die (Titan-)Platte durch Aussparungen an der Unterseite nur zu circa 50\% auf dem Periost aufliegt. So wird das Periost besser durchblutet und die Heilung gefördert. Manche Platten verfügen über ein zusätzliches Gewinde für den Schraubkopf und bieten somit Winkelstabilität. Eine weitere Verbesserung der Methode wird erreicht über die anatomische Ver- bzw. Vorformung der Platte und durch die Technik der Tunnelung (MIPPO = minimiert invasive perkutane Plattenosteosynthese). Dabei wird die Platte über eine Stichinzision submuskulär entlang des Knochens eingeschoben und über der Fraktur positioniert. Proximal und distal werden perkutan Schrauben zur Fixierung eingedreht. Sind die Schrauben eher frakturfern positioniert und nur in einer Kortikalisseite eingebracht, kann die Platte schwingen und die Funktion eines „Fixateurs interne“ übernehmen (Ausführungen 
beziehen sich auf (Müller 2011; Biberthaler und Oestern 2012; Marzi 2011; Bühren et al. 2016).

Postoperativ ist die Fraktur bewegungsstabil versorgt, jedoch nicht belastungsstabil (von Laer et al. 2007). Außerdem ist nach circa einem Jahr ein Zweiteingriff zwecks Metallentfernung notwendig.

Falls die Operation nicht minimalinvasiv durchgeführt werden kann, muss offen operiert werden, wobei das einer "biologischen Osteosynthese“ nicht gerecht würde. Danach soll die Blutversorgung des Periosts geschont werden und der „kleinstmögliche Eingriff" stattfinden (Müller 2011). Bei Missachtung dieses Prinzips kann es zu verzögerter Frakturheilung oder gar zu Pseudarthrose kommen.

Abbildung 16 zeigt Röntgenaufnahmen eines Patienten, dessen Fraktur mit einer Plattenosteosynthese versorgt wurde.

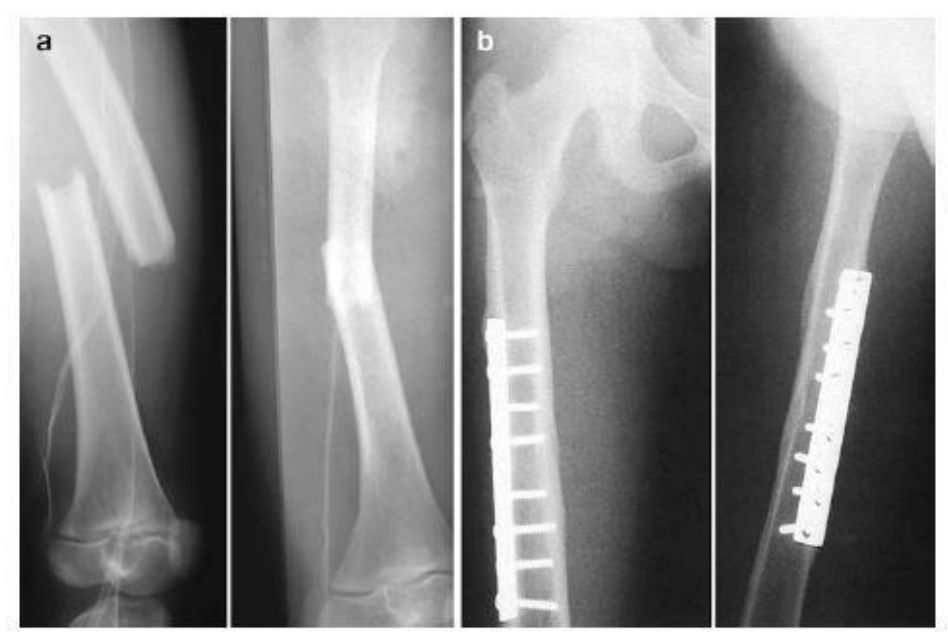

Abbildung 15: Femurschaftfraktur eines neunjährigen Jungen, a-b) Unfallbild, c-d) Versorgung mit einer Plattenosteosynthese

\subsubsection{Fixateur externe}

Die Indikation für diese minimalinvasive Therapie kann laut von Laer et al. (2007) bei instabilen Frakturen des Femurschaftes gestellt werden. Die Autoren beziehen sich speziell auf die Spiral- und Mehrfragmentfraktur, während Marzi (2010) noch die Trümmerfraktur hinzuzählt. Wenn die Fraktur besonders schnell versorgt werden muss (Polytrauma) oder ein hochgradiger Weichteilschaden vorliegt, wird der Fixateur externe favorisiert.

Die postoperativen Vorteile dieser Behandlungsmethode sind die sofortige Belastungsstabilität und das Entfallen eines Zweiteingriffes (der Fixateur kann ohne Narkose entfernt werden) (von Laer et al. 2007). 
Beim Erwachsenen wird der Fixateur externe als temporäre Maßnahme genutzt, beim Kind hingegen verläuft die Frakturheilung so rasch, dass die Ausbehandlung im Fixateur externe möglich ist (Bühren et al. 2016; Biberthaler und Oestern 2012).

Abbildung 11 zeigt Röntgenbilder eines dreijährigen Patienten, dessen Fraktur mit einem Fixateur externe stabilisiert wurde.

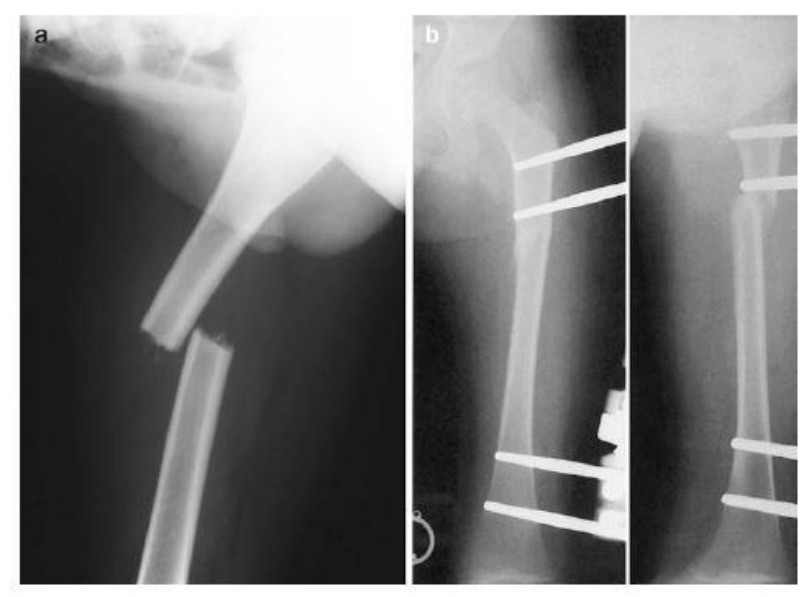

Abbildung 16: Femurschaftfraktur eines dreijährigen Jungen, a) Unfallbild, b) Versorgung mit dem Fixateur externe

\subsubsection{Marknagel}

Die Behandlung von Kindern mit dem Marknagel ist Ausnahmefällen vorbehalten, da die Gefahr einer Fugenschädigung besteht und in der Folge Wachstumsstörungen hervorgerufen werden können. Bei adipösen (>60 kg) und/oder großen (>1,60 m) Kindern würde die intramedulläre Schienung mit elastischen Nägeln (ESINs) jedoch keine ausreichende Stabilität ergeben und so muss auf den Fixateur externe oder Marknagel zurückgegriffen werden. In einem solchen Fall ist ein Marknagel speziell für Heranwachsende erhältlich: Adoleszentennagel (ALFN). Der Nagel sollte den Markraum nicht vollständig ausfüllen (Dietz et al. 2011). Der Vorteil dieser operativen Therapie ist die Belastungsstabilität nach nur wenigen Tagen, trotzdem muss eine gewichtsadaptierte Thromboseprophylaxe angedacht werden. Die Patient(inn)en und Eltern müssen über das Risiko einer Lungenembolie (durch Einschwemmungen aus dem Markraum in die Lunge) und einer verzögerten Frakturheilung aufgeklärt werden (Marzi 2011).

Die Leitlinie der deutschen Gesellschaft für Kinderchirurgie (2014) empfiehlt die Anwendung eines ALFN in Einzelfällen bereits ab einem Gewicht von $50 \mathrm{~kg}$, weist jedoch auch auf die schwerste Komplikation hin - die avaskuläre Femurkopfnekrose (http://www.awmf.org/leitlinien/detail/II/006-016.html). 
Trotz der Gefahr dieser katastrophalen Komplikation mit der Konsequenz eines künstlichen Hüftgelenks im Kindesalter sprechen jüngere Studien hingegen von sehr guten Behandlungsergebnissen. Keeler et al. (2009) berichten in ihrer Studie mit 78 Patient(inn)en (Alter acht bis 18 Jahre, Durchschnittsgewicht $70 \mathrm{~kg}$ ) über gute Ergebnisse. Abgesehen von zwei Patient(inn)en, die eine Infektion erlitten, blieben Komplikationen aus.

Reynolds et al. (2012) verglichen in einer Kohorte von 22 Patient(inn)en die ESIN- mit der ALFN-Therapie hinsichtlich des Zeitraums bis zur Mobilität sowie die Komplikationsrate (Altersspanne zehn bis 17 Jahre, Gewicht ESIN-Gruppe 46 kg, Gewicht ALFN-Gruppe $60 \mathrm{~kg}$ ). Patient(inn)en mit ALFN konnten signifikant schneller ihr Bein voll belasten (ALFN Patient(inn)en nach vier Wochen, ESIN-Patient(inn)en nach neun Wochen). Unterschiede in der Komplikationsrate waren nicht signifikant. Der ALFN sei demnach für schwerere und/oder ältere Kinder vorteilhafter als die elastisch stabile intramedulläre Nagelung.

Wird der ALFN über einen lateralen Eintrittspunkt in den Femurschaft eingeführt (und nicht über die Fossa piriformis oder die Apex des Trochanter majors), ist laut Martus (2016) mit hervorragenden funktionalen Langzeitergebnissen zu rechnen. In seiner Studie mit 426 Patient(inn)en erlitt keine bzw. keiner eine Osteonekrose. Martus (2016) empfiehlt die Verwendung einer rigiden intramedullären Marknagelung für Patien$\mathrm{t}$ (inn)en ab elf Jahren, die schwerer als 49 kg oder für ihr Alter hoch gewachsen sind.

\subsection{Ziele der Behandlung der kindlichen Femurschaftfraktur und Prognose}

Das Therapieziel kindlicher Femurschaftfrakturen ist eine primär definitive, achsengerechte, möglichst übungsstabile Versorgung. Eine primär definitive Versorgung ist anzustreben, um erneute Manipulationen und somit die Stimulation der Wachstumsfugen zu vermeiden und um ggf. dem Kind unnötige Narkosen zu ersparen. Auch wenn das kindliche Skelett über das Potenzial einer Spontankorrektur verfügt, sollten nach dem zehnten Lebensjahr keine Fehlstellungen mehr belassen werden. Die Korrekturmechanismen können als „Ergänzung“ in den Therapieplan einfließen, jedoch ist ihr Ausmaß nicht exakt vorhersehbar.

Die Ausheilungsprognose für Kinder mit einer Femurschaftfraktur ist exzellent. Die häufigste langfristige Komplikation ist eine Beinverlängerung von über $2 \mathrm{~cm}$ durch die frakturbedingte Mehrdurchblutung und das Mehrwachstum in den zwei Jahren nach Fraktur. In der Literatur schwanken die Angaben über diskrepante Beinlängen - im 
Mittel liegt die Rate bei 6,5\% (Angaben zwischen 0 - 47\%). Rotationsfehler sind eine weitere Komplikation, sie treten in der Literatur in 18\% der Fälle auf (Gresing 2011).

\subsubsection{Spontankorrektur}

Das kindliche Skelett besitzt die einzigartige Fähigkeit, im Laufe des Wachstums über vermehrten An- und Abbau von Knochengewebe Achsabweichungen ausgleichen zu können - dieser Vorgang wird unter dem Begriff "Spontankorrektur" zusammengefasst. Dabei spielen zwei Mechanismen in der Heilungsphase eine Rolle: das Remodelling der Meta- und Diaphyse, das vom periost-endostalen System geleistet wird, und das asymmetrische epiphysäre Wachstum der Fuge senkrecht zur Belastungsebene. Die Vorgänge finden parallel statt und "scheinen voneinander abhängig zu sein“ (von Laer 2010).

Beim Remodelling im Schaftbereich wird auf der druckbelasteten, konkaven Knochenseite Periost angebaut, während auf der zugbelasteten, konvexen Seite endostales Knochengewebe resorbiert wird. Dies geschieht nach dem Roux-Gesetz (,Minimum an Material für ein Maximum an Belastbarkeit", von Laer et al. 2007) und dem WolffTransformationsgesetz („optimale Balance zwischen Form und Funktion“, Schmittenbecher 2011).

Bei Seit-zu-Seit-Verschiebungen reicht allein dieser Mechanismus aus, um die Knochengestalt wie vor dem Frakturereignis wiederherzustellen. Tritt jedoch ein Achsenknick in der Frontal- oder Sagittalebene auf, kommt ein zusätzlicher Korrekturmechanismus zum Tragen. Die schräg gestellte Epiphyse richtet sich durch asymmetrisches Längenwachstum orthograd zur Belastungsebene aus: Über vermehrten Druck wird die Konkavseite der Fuge zu appositionellem Mehrwachstum angeregt und somit die Ausrichtung der Wachstumsfuge korrigiert (vgl. Abbildung 17 und Abbildung 18). 


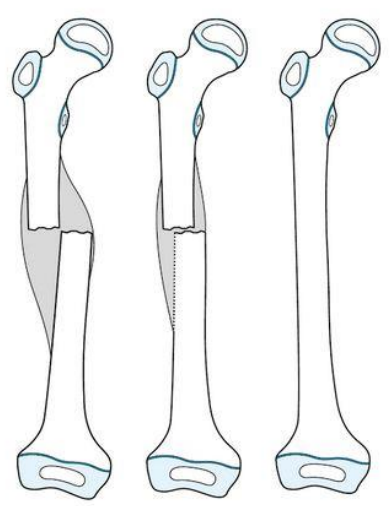

Abbildung 17: Korrektur einer Seit-zu-Seit Fehlstellung

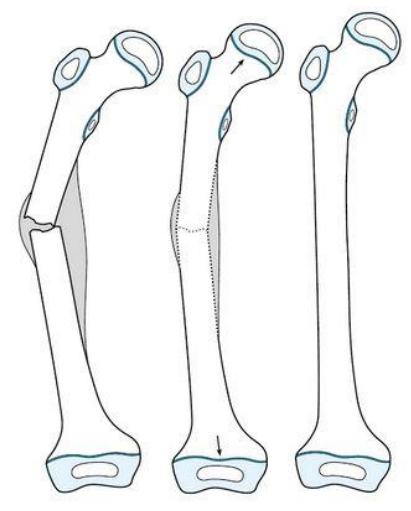

Abbildung 18: Kombination aller Mechanismen der Spontankorrektur, die den Ausgleich eines Achsenknicks ermöglicht

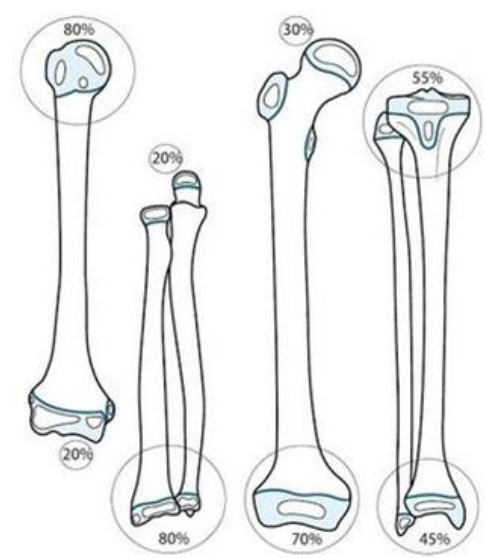

Abbildung 19: Wachstumsanteil der einzelnen Fugen

Das Korrekturpotenzial der jeweiligen Frakturstelle ist somit unter anderem bestimmt durch die Entfernung und den Wachstumsanteil der nächstgelegenen Wachstumsfuge. Die distale Wachstumsfuge des Femurs übernimmt 70\% des Wachstums dieses Knochens (Abbildung 20) und somit haben Frakturen in ihrer Nähe ein hervorragendes Korrekturpotenzial. Am proximalen Femurpol hingegen besteht ein nur mäßiges Korrekturpotenzial, der Wachstumsanteil dieser Fuge liegt bei 30\%. Generell werden Achsfehler der unteren Extremität besser korrigiert als die der oberen.

Es gibt noch weitere Faktoren, die die Spontankorrektur beeinflussen:

- das Patient(inn)enalter bei Frakturereignis (Je jünger der Patient bzw. die Patientin ist, desto eher erfolgt Korrektur.)

- umliegende Muskulatur (Je mehr die Frakturzone funktionell belastet wird, desto besser die Korrektur.)

- $\quad$ angrenzende Gelenke (Je multiplaner das angrenzende Gelenk, desto größer ist das Korrekturpotenzial.)

- Ausmaß der Achsabweichung (Je größer der Achsfehler, desto zuverlässiger ist eine Korrektur.)

- Fugenschluss-Zeitpunkt der nächstgelegenen Wachstumsfuge (Je langlebiger, desto sicherer ist eine Korrektur. Die Fuge des proximalen Femurs verschließt sich bis zum 17., die distale Fuge bis zum 18.Lebensjahr (Schmittenbecher 2011).

- Ebene des Achsfehlers (Sagittale Abweichungen werden besser korrigiert als Achsfehler in der Frontalebene.)

- Varusfehler (Achsabweichung nach innen, Knochen nach außen gebogen) werden besser korrigiert als Valgusdeformitäten (Achsabweichung nach außen, Knochen nach innen gebogen). Am Oberschenkel ist dies durch die Anordnung der Muskelgruppen zu erklären. Böhm (2007) stellt fest, dass Varusfehlstellungen des Femurs, die sich grundsätzlich gut zurückbilden, umso langsamer korrigiert würden, je proximaler sie liegen. 
Bei besonders ausgeprägten korrigierenden Umbauvorgängen im Epiphysenbereich kann es durch eine langandauernde Mehrdurchblutung auch zu einem gleichmäßigen Längenwachstum kommen (auf der frakturierter Seite). So können Beinlängenunterschiede entstehen oder sich im besten Falle vorher bestandene Alterationen der Beinlänge ausgleichen. Die Auswirkung von solchen Wachstumsstörungen hängt vom Patient(inn)enalter zum Unfallzeitpunkt ab: Bis zum zehnten Lebensjahr erfolgt ein überschießendes Wachstum und das Bein verlängert sich. Jenseits des zehnten Lebensjahres reagiert die Fuge auf Stimulation mit vorzeitigem Verschluss und es resultiert eine Beinverkürzung.

Die Gefahr einer Beinverlängerung oder -verkürzung der verletzten Seite durch Hyperperfusion und somit einer Stimulation der Wachstumsfuge sollte bei der Wahl der Therapie bedacht werden. So sind große Achsabweichungen, auch wenn sie im Laufe des Wachstums spontan korrigiert würden, von außen auszugleichen um unterschiedlichem Beinlängenwachstum vorzubeugen. „Je mehr und länger Remodellierung, desto mehr Beinlängendifferenz" (von Laer et al. 2007).

Um diese Komplikation zu vermeiden, sollte eine zügige und definitive Frakturversorgung vorgenommen werden. Definitiv auch, um einem Therapiewechsel nach dem fünften bis siebten Tag vorzubeugen. Dieser würde mit einer erneuten starken Stimulation des Kallus einhergehen und die Gefahr einer Wachstumsstörung erhöhen. Falls im Rahmen der Behandlung eine Beinlängendifferenz auftritt, so beträgt sie meist nicht mehr als $2 \mathrm{~cm}$ und ist konservativ gut mit z.B. Absatzerhöhungen auszugleichen. $A b$ einer Differenz von über $3 \mathrm{~cm}$, wäre eine operative Korrektur (eine Verlängerungsosteotomie) anzustreben (Böhm 2007).

Spontankorrekturen von Rotationsfehlern finden nicht über einen eigenen Mechanismus, sondern lediglich im Rahmen der physiologischen Detorsion und damit ungezielt und über einen langen Zeitraum statt. Achsfehler in der Rotationsebene äußern sich als Innen- oder Außenrotationsfehler des Beines. Am Oberschenkelknochen kann die Achsabweichung sehr gut vom Hüftgelenk kompensiert werden, sodass die Patient(inn)en während der Jahre des Wachstums und der Spontankorrektur keine Einschränkung spüren (siehe Abbildung 20). 


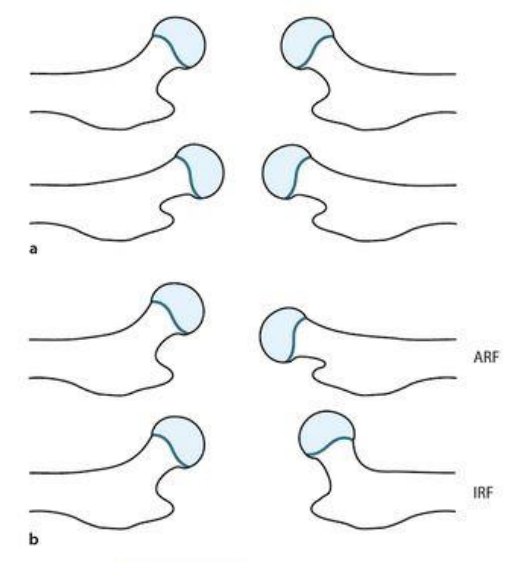

Abbildung 20: Spontankorrektur von Rotationsfehlern

a) zeigt die physiologische Detorsion der Oberschenkelhälse, die sich ab der Geburt bis zur Vollendung des Wachstums vollzieht - von $35^{\circ}$ auf $15^{\circ}$.

b) Kommt es bei einer Fraktur zu einem Außenrotationsfehler (ARF) durch das distale Fragment, so wird die physiologische Derotation „vorweggenommen“ und werden die Derotationsvorgänge der Schenkelhälse dieses Beines weniger ausgeprägt ausfallen als auf der Gegenseite. ARF machen 70\% der Rotationsfehler aus. Liegt ein Innenrotationsfehler (IRF) vor, so ist der Schenkelhals der betroffenen Seite stärker rotiert, eventuell sogar um mehr als $35^{\circ}$. Im Laufe des Wachstums, würde es auf der Frakturseite zu vermehrten Detorsionsvorgängen kommen um einen Ausgleich anzustreben.

Die Ausführungen beziehen sich auf (von Laer et al. 2013; Schmittenbecher 2011; Gresing 2011; von Laer 2010; Böhm 2007).

\subsubsection{Tolerable Fehlstellung nach Böhm}

Böhm (2007) definiert Dislokations-Werte nach Patient(inn)enalter und Fehlstellungsart für Femurfrakturen, die als tolerabel gelten. Liegt eine Achsabweichung außerhalb dieser Grenzwerte vor, sollte eingegriffen und die Fehlstellung korrigiert werden (geschlossene/offene Reposition). Ist die Fraktur bereits konsolidiert, und es liegt noch eine tolerable dislozierte Achsfehlstellung vor, wird das Kind keine funktionellen Einschränkungen erfahren. Sind die Dislokationen jedoch intolerabel, so muss mit Wachstumsstörungen, Gangstörungen durch Abweichungen der Knieachse, Coxa valga, Hüftgelenksarthrose im jungen Erwachsenenalter, Beckenschiefstand und eventuell mit Skoliose gerechnet werden (Böhm 2007) (vgl. Abbildung 21). 


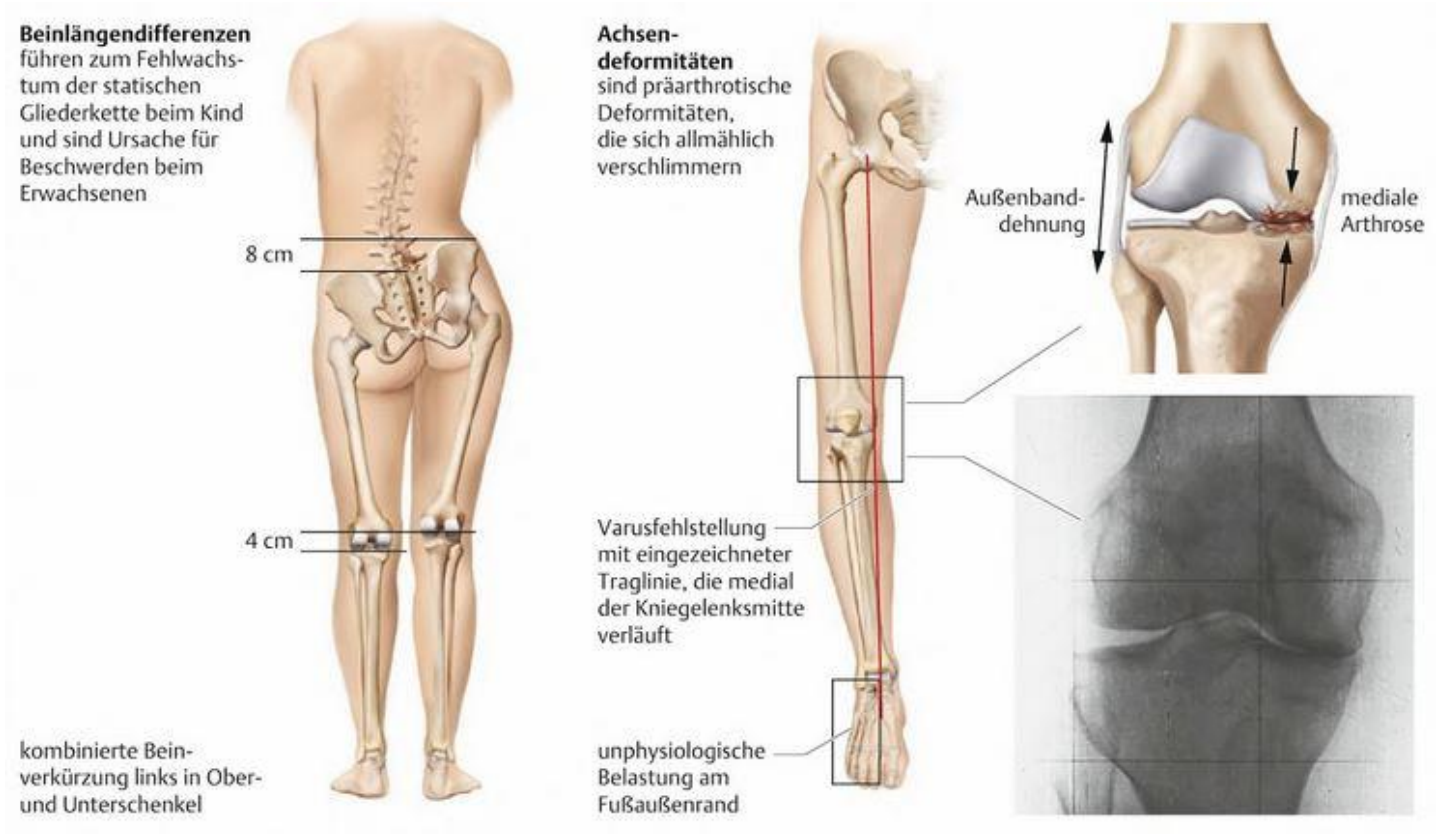

Abbildung 21: Mögliche Folgen von stimulativen Wachstumsstörungen: Beckenschiefstand, kompensatorische Skoliose und prä-arthrotische Hüftgelenksschäden.

Von Laer et al. (2007) unterscheiden zwischen proximalen und diaphysären Femurschaftfrakturen. Bei proximalen Schaftfrakturen sieht er keine Toleranz für Achsabweichungen da keine Spontankorrektur stattfinde. Bei diaphysären Oberschenkelfrakturen definiert er die Grenzen, wie in Tabelle 2 dargestellt.

Tabelle 2: Grenzwerte für „tolerabel dislozierte" Achsabweichungen nach von Laer et al. (2007)

\begin{tabular}{|l|l|l|}
\hline Art der Fehlstellung & \multicolumn{2}{|l|}{ Grenzwerte, in denen Fehlstellungen „tolerabel disloziert“ sind } \\
\hline & bis zum dritten Lebensjahr & jenseits des dritten Lebensjahres \\
\hline Seit-zu-Seit-Verschiebung & bis ganze Schaftbreite & bis maximal halbe Schaftbreite \\
\hline Verkürzung & $1-2 \mathrm{~cm}$ & keine Toleranz \\
\hline Varus & bis $20^{\circ}$ & $10^{\circ}$ \\
\hline Valgus & bis $10^{\circ}$ & bis $10^{\circ}$ \\
\hline Antekurvation & bis $10^{\circ}$ & bis $10^{\circ}$ \\
\hline Rekurvation & keine Toleranz & keine Toleranz \\
\hline Rotationsfehler & bis $20^{\circ}$ & bis $10^{\circ}$ \\
\hline
\end{tabular}




\section{Material und Methoden}

\subsection{Patient(inn)enkollektiv}

Die vorliegende Arbeit stützt sich auf Daten von 101 Kindern und Heranwachsenden, die aufgrund von Femurschaftfrakturen (insgesamt 103) in der Universitätsmedizin Göttingen sowie im Universitätsklinikum Magdeburg behandelt wurden. Zur retrospektiven Datenanalyse konnten Patient(inn)enakten folgender Zeiträume ausgewertet werden:

Universitätsmedizin Göttingen - 01.01.2006 bis 01.11.2013

Universitätsklinikum Magdeburg - 01.05.2003 bis 31.12.2010.

Um in das Patient(inn)enkollektiv aufgenommen zu werden, durften die Erkrankten zum Zeitpunkt der Frakturversorgung nicht älter als 16 Jahre sein. Weitere Ausschlusskriterien bestanden nicht.

Es wurden alle Datensätze aus den Krankenakten einbezogen, auch unvollständige, damit die Versorgungsrealität so exakt wie möglich abgebildet wird.

\subsection{Datenerfassung}

Die Bescheinigung der Ethikkommission der Universitätsmedizin Göttingen für die ethische und rechtliche Unbedenklichkeit der Datenerhebung zur Erstellung dieser Arbeit wurde eingeholt. Am 07.11.2013 bewilligte der Datenschutzbeauftragte des Archivs der Universitätsmedizin Göttingen einen Datenzugriffsantrag, sodass ab Dezember 2013 die Patient(inn)enakten für die Datenanalyse ausgewertet werden konnten. Beim Sichten der Akten und Kodieren der Parameter wurden die Daten anonymisiert und standardisiert erfasst. Dabei sind alle Datenschutzmaßnahmen eingehalten worden.

Für die präzise Beschreibung des Patient(inn)enkollektivs wurden folgende Parameter erfasst: Alter und Geschlecht der Erkrankten, Body-Mass-Index, Vor- und Begleiterkrankungen, Unfallgeschehen, Seite des frakturierten Beines, Frakturtyp und Angabe, ob eine pathologische Fraktur vorlag. Unfallgeschehen und Frakturtyp wurden jeweils kategorisiert und sind in Tabelle 3 dargestellt. 
Tabelle 3: Unfallgeschehen

\begin{tabular}{|ll|}
\hline Kategorie & Unfallgeschehen \\
1 & Sturz $\leq 1 \mathrm{~m}$ \\
2 & Sturz $>1 \mathrm{~m}$ \\
3 & Sturz $\geq 3 \mathrm{~m}$ \\
4 & Fahrrad \\
5 & Fußball \\
6 & Pferdesport \\
7 & sonstiger Sport \\
8 & Toben/Spielen \\
9 & Pkw-/Businsasse \\
10 & Verkehrsunfall Fußgänger(in) gegen Pkw/Motorrad \\
11 & Verkehrsunfall Fahrradfahrer(in) gegen Pkw/Motorrad \\
12 & tätliche Auseinandersetzung /Gewalt \\
13 & "umgeknickt“/Distorsion \\
14 & Anpralltrauma inkl. "gegen etwas getreten/geschlagen“ \\
15 & Quetschtrauma inkl. Gegenstände /Personen, die auf Patient(inn)en \\
& gefallen sind \\
16 & kein Trauma erinnert/beobachtet \\
\hline
\end{tabular}

Zur Einordnung des Frakturtyps wurde in den Patient(inn)enakten die Befundung der radiologischen Abteilungen herangezogen und, falls operiert wurde, mit dem Operationsbericht abgeglichen. In den wenigen Fällen, bei denen die Angaben zum Frakturtyp nicht übereinstimmten, wurde für die Datenanalyse die Angabe des Operateurs übernommen. Jede Fraktur wurde jeweils nur einer Kategorie zugeordnet (vgl. Tabelle 4).

Tabelle 4: Kategorien der Frakturtypen

\begin{tabular}{|ll|}
\hline Kategorie & Frakturtyp \\
1 & Querfraktur \\
2 & Schrägfraktur \\
3 & Spiralfraktur \\
4 & Trümmerfraktur \\
5 & Mehrfragmentfraktur \\
6 & nur "Femurschaftfraktur“ \\
7 & nur "Femurfraktur“ \\
\hline
\end{tabular}

Um eine breite Datenbasis zu erstellen und damit mögliche Zusammenhänge feststellen zu können, wurden zudem das Entlassungsdatum der Erkrankten, der Zeitpunkt der Operation zur Implantatentfernung, intra- und postoperative Komplikationen sowie die Dicke, Länge und Anzahl von operativ eingeführten bzw. angebrachten Kraftträgern ermittelt. Dazu zählen Kirschner-Drähte, Hohlschrauben, 
Fixateur externe, Platten, Marknägel und elastisch stabile intramedulläre Nägel (ESINs).

Das Hauptaugenmerk der Arbeit ist auf die Erörterung der postoperativen Komplikationen nach Einsetzen von ESINs gerichtet. Aus diesem Grund wurde eine intensive Recherche zu möglichen postoperativen Komplikationen bei dieser Technik betrieben und eine Liste erstellt (siehe Tabelle 5). Litten die Patient(inn)en unter mehr als einer Komplikation, so wurde nicht priorisiert, sondern alle Komplikationen wurden gleichermaßen gewichtet. Für außergewöhnliche oder nicht zuzuordnende Komplikationen wurde ein Extrafeld „Bemerkungen“ erstellt.

Tabelle 5: Mögliche postoperative Komplikationen

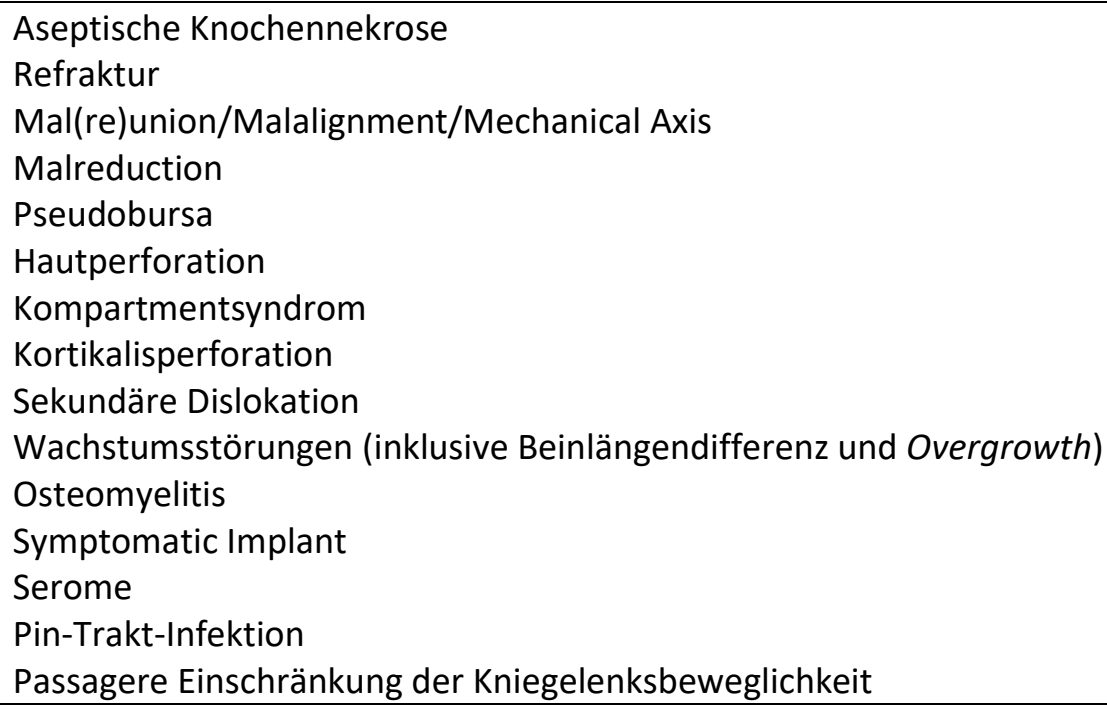

Tabelle 6 zeigt, aus welchen Dokumenten der Patient(inn)enakten die oben genannten Parameter und Daten für die Analyse entnommen wurden. 
Tabelle 6: Analysedaten und Dokumente der Patient(inn)enakten

\begin{tabular}{|l|l|}
\hline Daten für die Analyse & Dokument in der Patient(inn)enakte \\
\hline Geburtsdatum & Patient(inn)enstammdatenbogen \\
\hline Geschlecht & Patient(inn)enstammdatenbogen \\
\hline Gewicht & Formularset Anästhesie \\
\hline Größe & Formularset Anästhesie \\
\hline $\begin{array}{l}\text { Art und Höhe der Fraktur, } \\
\text { frakturierte Seite }\end{array}$ & $\begin{array}{l}\text { schriftlicher radiologischer Befund } \\
\text { Operationseingriffsprotokoll } \\
\text { Operationsbericht }\end{array}$ \\
\hline pathologische Fraktur & Brief des Arztes/der Ärztin bei Entlassung \\
\hline Datum Beginn Liegedauer & $\begin{array}{l}\text { Brief des Arztes/der Ärztin bei Entlassung } \\
\text { Aufnahmebogen }\end{array}$ \\
\hline Datum Ende Liegedauer & Brief des Arztes/der Ärztin bei Entlassung \\
\hline Unfallhergang und Begleitverletzungen & $\begin{array}{l}\text { Kurzbrief des Arztes/der Ärztin } \\
\text { Aufnahmebogen } \\
\text { Arztbrief bei Entlassung }\end{array}$ \\
\hline Reposition offen oder geschlossen & $\begin{array}{l}\text { Operationseingriffsprotokoll } \\
\text { Operationsbericht }\end{array}$ \\
\hline $\begin{array}{l}\text { eingeführte Kraftträger (Länge, Dicke, Anzahl, } \\
\text { Material) }\end{array}$ & $\begin{array}{l}\text { Operationseingriffsprotokoll } \\
\text { Operationsbericht }\end{array}$ \\
\hline intraoperative Komplikationen & $\begin{array}{l}\text { Operationseingriffsprotokoll } \\
\text { Operationsbericht }\end{array}$ \\
\hline Stellung der Fraktur postoperativ & $\begin{array}{l}\text { Operationseingriffsprotokoll } \\
\text { Operationsbericht }\end{array}$ \\
\hline Zeitpunkt der Metallentfernung & $\begin{array}{l}\text { Verlaufsbogen } \\
\text { Brief des Arztes/der Ärztin bei Entlassung }\end{array}$ \\
\hline $\begin{array}{l}\text { postoperative Komplikationen und Zeitpunkerand } \\
\text { des Auftretens }\end{array}$ & $\begin{array}{l}\text { Verlaufsbogen } \\
\text { Brief des Arztes/der Ärztin bei Entlassung }\end{array}$ \\
\hline Nachbehandlung & Brief des Arztes/der Ärztin bei Entlassung \\
\hline Begleiterkrankungen & schriftlicher radiologischer Befund \\
\hline Ergebnis nach Abschluss der Behandlung & \\
\hline
\end{tabular}

Um festzustellen, wie sich die postoperativen Komplikationen und die vorgenannten Aspekte bedingen, wurden von den Patient(inn)en, denen ESINs implantiert worden waren, Röntgenbilder ausgewertet. Es wurde der Durchmesser des Markraums proximal und distal von der Fraktur in Millimetern gemessen. So wurden Informationen über das Verhältnis von Markraumdurchmesser zur Dicke des ESINs gewonnen. Die Ausmessungen der Röntgenbilder wurden mit dem „Picture Archiving and Communication System“- Programm „Centricity“ (der Firma GE Healthcare, Milwaukee, USA) in Göttingen und am Universitätsklinikum Magdeburg mit dem Programm CHILI - Digital Radiology (der Firma Chili GmbH, Sitz in Dossenheim) durchgeführt. 


\subsection{Datenverarbeitung}

Die gewonnenen Daten wurden mit dem Programm Excel 2013 notiert und ausgewertet. Um die Textform dieser Arbeit zu erstellen, wurde das Programm Word 2013 (beide Bestandteil von Microsoft Office Professional Plus von Microsoft, Redmond, USA) genutzt.

Die statistische Auswertung und grafische Darstellung der Ergebnisse wurde mit dem Statistik-Programm Stata/SE 13.1 for Windows (StataCorp LP, College Station, USA) durchgeführt. Es erfolgten Signifikanztests, bei denen das Signifikanzniveau bei $p<0,05$ lag. Die Tests umfassten t-Tests, logistische, multivariable sowie Kernel-Regressionen.

Die erhobenen Daten von Gewicht und Körpergröße wurden genutzt, um den BodyMass-Index (Einheit $\mathrm{kg} / \mathrm{m}^{2}$ ) zu erstellen. Dafür wurde das Körpergewicht dividiert durch die Körpergröße im Quadrat. Um die Body-Mass-Indexe der Kinder trotz ihres unterschiedlichen Alters miteinander vergleichen zu können, wurden Perzentilkurven zu Hilfe genommen. Die „Perzentilkurven für den Body Mass Index (Jungen 0 - 18 Jahre)“ sowie die „Perzentilkurven für den Body Mass Index (Mädchen 0 - 18 Jahre)“ stellte die Kinderklinik Göttingen zur Verfügung. Es mussten jeweils der Body-MaßIndex sowie das Alter aufgetragen werden um eine Perzentile ablesen zu können. 


\section{$3 \quad$ Ergebnisse}

\subsection{Patient(inn)enkollektiv: Geschlecht, Alter, Body-Mass-Index, Perzentile, Frakturtyp, frakturierte Seite}

Das der Arbeit zugrunde liegende Patient(inn)enkollektiv bestand aus 101 Patient(inn)en mit Femurschaftfraktur und setzte sich aus 67,33\% (68/101) männlichen und $32,67 \%$ (33/101) weiblichen Kindern zusammen. Sie wurden in den Universitätsklinika Göttingen und Magdeburg behandelt.

Das Durchschnittsalter aller Patient(inn)en lag bei 7,05 Jahren. Patientinnen hatten ein durchschnittliches Alter von 7,09 Jahren und lagen damit über dem Durchschnitt. Patienten mit 7,04 Jahren lagen knapp darunter. Der bzw. die jüngste Erkrankte war drei Monate alt und der bzw. die älteste war 15 Jahre und sieben Monate. Ein Drittel (26/84) aller Frakturen ereignete sich bei Kindern im Alter von zwei bis vier Jahren (Abbildung 22).

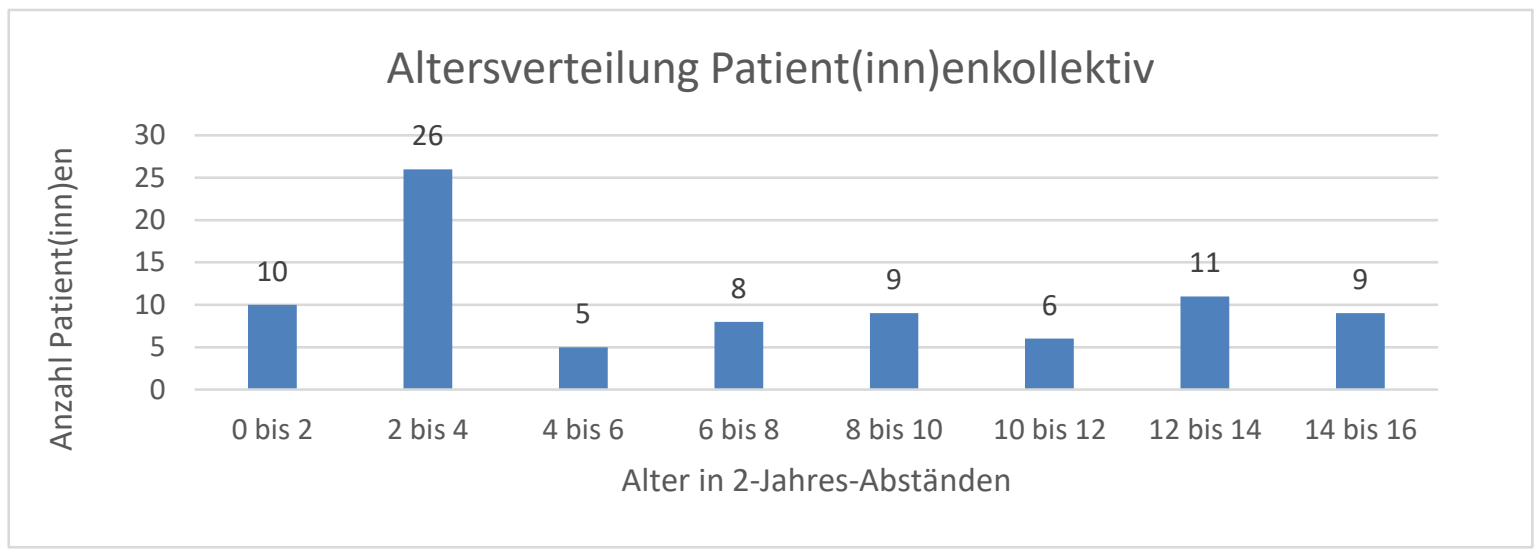

Abbildung 22: Altersverteilung im Patient(inn)enkollektiv in 2-Jahres-Abständen

Das Verhältnis Gewicht zu Körpergröße, ausgedrückt mit dem Body-Mass-Index, rangierte zwischen $8,0 \mathrm{~kg} / \mathrm{m}^{2}$ und $38,7 \mathrm{~kg} / \mathrm{m}^{2}$. Ein Viertel der Kinder $(17 / 66)$ hatte den Body-Maß-Index 14-16 kg/m², was dem Normalgewicht (Perzentile 50) eines siebenjährigen Kindes (siehe Durchschnittsalter) entspricht. Der durchschnittliche Body-Maß-Index im Patient(inn)enkollektiv lag jedoch mit $17,9 \mathrm{~kg} / \mathrm{m}^{2}$ um fast zwei Punkte höher. Die Häufigkeitsverteilung des Body-Mass-Indexes im Patient(inn)enkollektiv ist in Abbildung 23 dargestellt. 


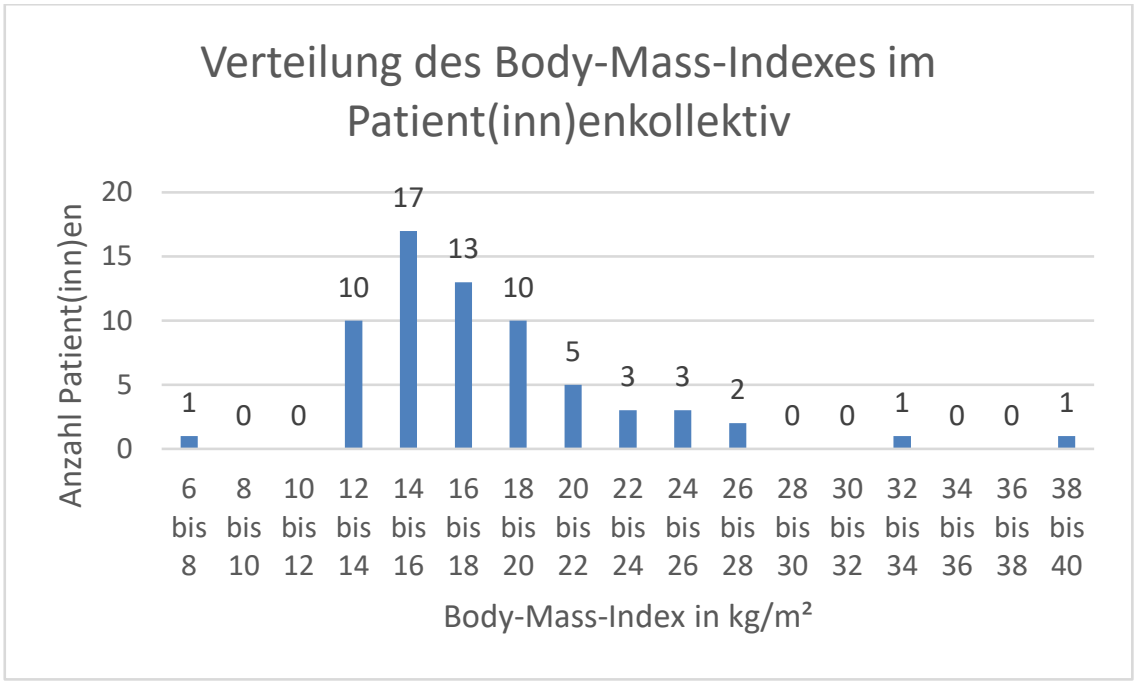

Abbildung 23: Body-Mass-Index des Patient(inn)enkollektivs

Um den Body-Mass-Index bei Kindern richtig zu bewerten, ist es wichtig, ihn im Kontext mit Kindern gleichen Alters, gleichen Geschlechts und gleicher Abstammung zu betrachten. Dafür wird der Body-Mass-Index auf Perzentilkurven aufgetragen. Abbildung 24 zeigt, wie viele Patient(inn)en auf jeder Perzentilkurve lagen.

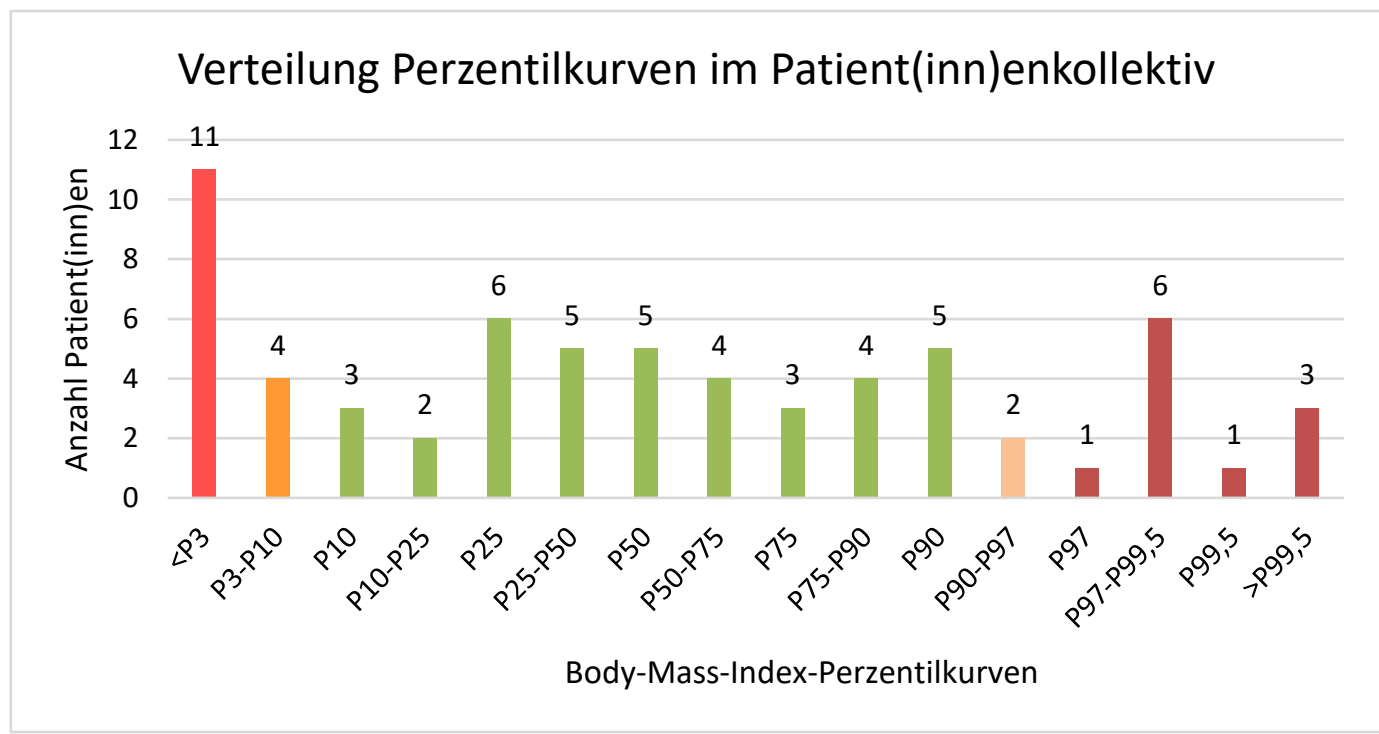

Abbildung 24: Anzahl Patient(inn)en pro Perzentilkurve für den Body-Mass-Index. Grün steht für Normalgewicht, Orange/Aprikot für Unter- bzw. Übergewicht, Rot für starkes Unter- bzw. Untergewicht.

Die Kategorisierung der Perzentilkurven ergab Folgendes: Mehr als die Hälfte der Kinder (56,92\% bzw. 37/65) war normalgewichtig. Vier von 65 Kindern $(6,15 \%)$ hatten Untergewicht und zwei von 65 (3,08\%) Übergewicht. Jeweils 16,92\% (11/65) machten die stark unter- bzw. stark übergewichtigen Patient(inn)en aus (siehe Abbildung 25). 


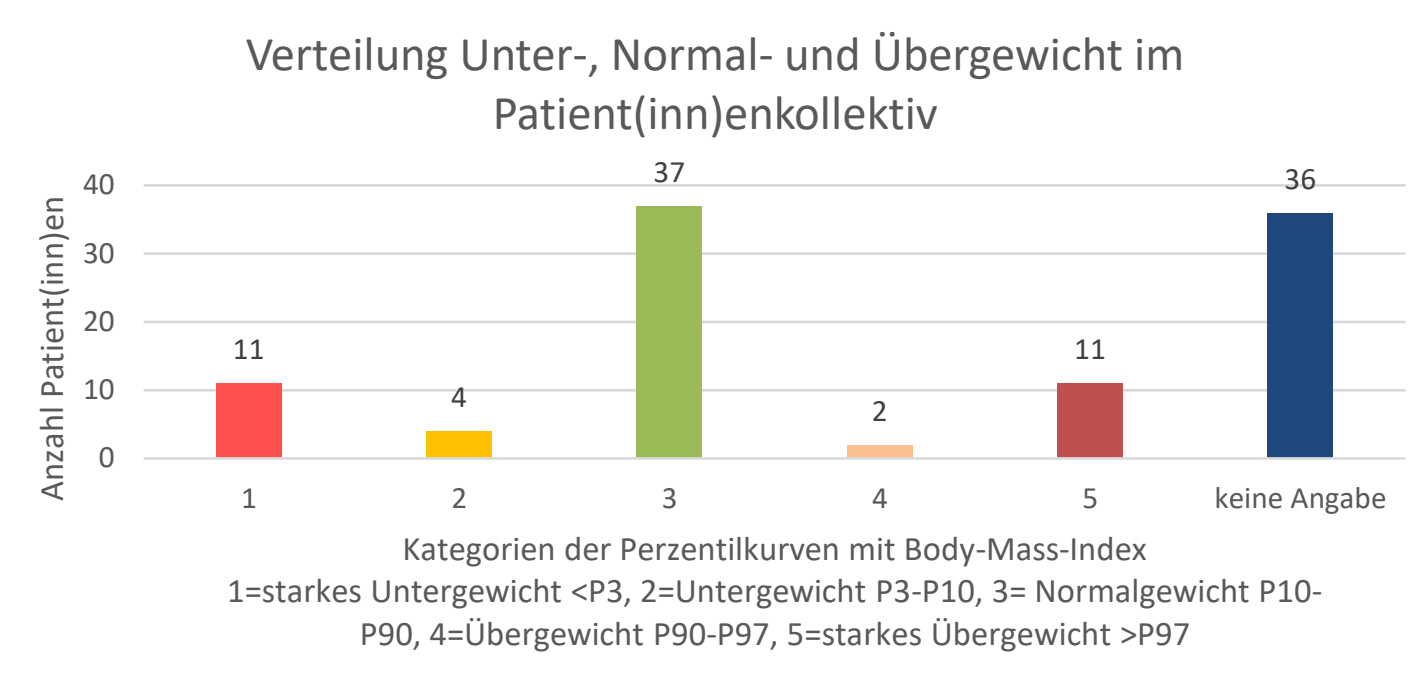

Abbildung 25: Verteilung von Unter-, Normal- und Übergewicht im Patient(inn)enkollektiv. Kategorisierung der Perzentilkurven: 1=starkes Untergewicht $\angle P 3,2=$ Untergewicht P3-P10, 3= Normalgewicht P10-P90, 4=Übergewicht P90,1-P97, 5=starkes Übergewicht >P97.

Tabelle 15 im Anhang gibt eine detaillierte Übersicht über Häufigkeiten der Unfallgeschehnisse je nach Body-Mass-Index der Patient(inn)en.

Der häufigste Frakturtyp war die Spiralfraktur. Knapp ein Drittel der Patient(inn)en (31,68\% bzw. 32/101) zog sich diese zu. An zweiter Stelle stehen mit gleicher jeweiliger Häufigkeit Quer- und Schrägfraktur; sie machen je 14,85\% (15/101) aus. In 7/101 Fällen und damit weitaus seltener traten Mehrfragmentfrakturen (drei bis sechs Fragmentstücke) auf. Dies entspricht 6,93\%. Eine Rarität stellte die Trümmerfraktur mit mehr als sechs Fragmentstücken dar (1/101).

Bei einem Drittel der Fälle waren die Angaben zur Frakturform ungenau: In 15,84\% der Fälle (16/101) war lediglich die Diagnose „Femurschaftfraktur" gestellt worden und in $14,85 \%$ der Fälle (15/101) lag ein Bruch vor, der keiner der sechs vorangegangenen Kategorien zugeordnet werden konnte. Beispiele dafür sind Angaben wie "Grünholzfraktur am distalen Femur", „offen dislozierte Oberschenkelfraktur [...]Konsolidierung im mittleren Drittel des Schafts", „geschlossene Fraktur im Bereich fibröser Dysplasie (mittig) proximaler li Oberschenkel“ oder „Oberschenkel-Stückbruch im mittleren Schaftdrittel“. Eine Darstellung der Werte ist in Abbildung 26 und die Häufigkeiten der Therapien je nach Frakturtypen sind im Anhang 6.4 (Tabelle 21) zu finden. 


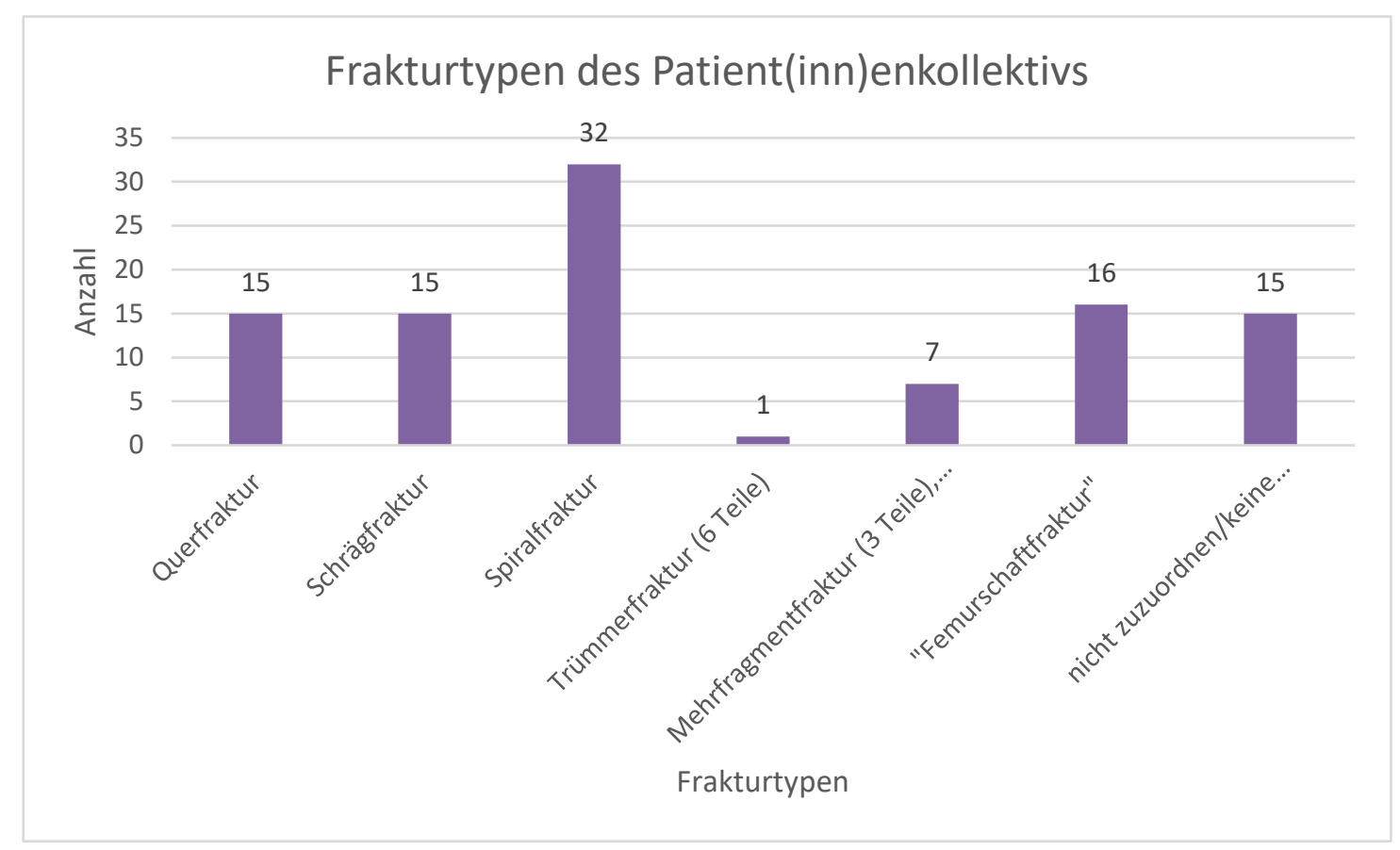

Abbildung 26: Häufigkeiten der verschiedenen Frakturformen

Bei 48 Patient(inn)en konnte ermittelt werden, ob sie sich das rechte oder linke Bein frakturiert hatten. Bei mehr als der Hälfte der Fälle (56,25\% bzw. 27/48) war das linke Bein gebrochen, das rechte Bein zu 39,58\% (19/48). Zwei Patient(inn)en hatten sich bei einem Verkehrsunfall beide Beine gebrochen (4,17\%). 


\subsection{Kontext des Frakturereignisses: Unfallgeschehen, Begleit- erkrankungen, pathologische Frakturen}

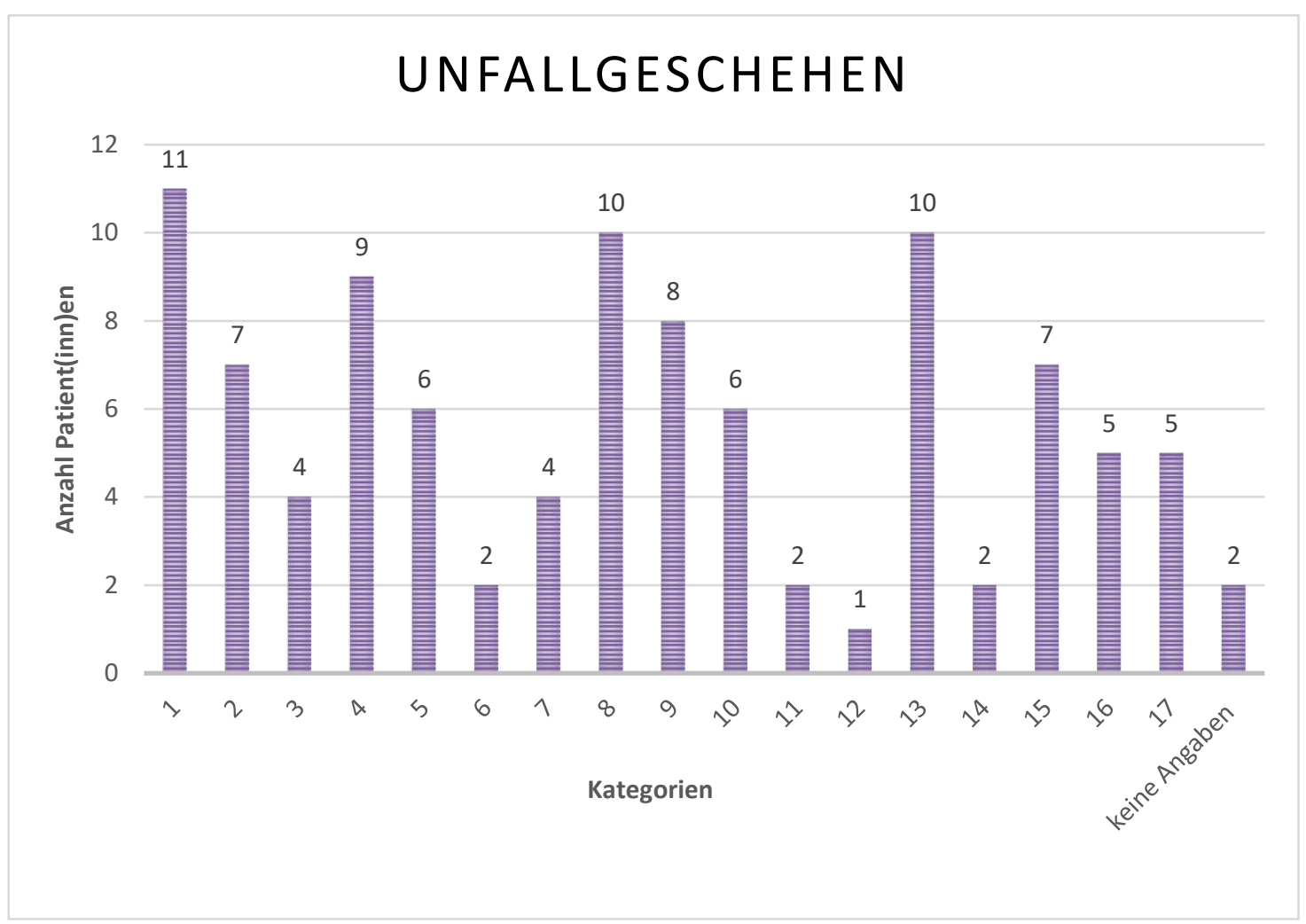

Abbildung 27: Unfallgeschehen. Es wird dargestellt, auf welche Art und Weise die Patient(in$\mathrm{n})$ en sich ihre Fraktur zuzogen. Die verschiedenen Unfallmechanismen wurden kategorisiert: 1 = Sturz $\leq 1 \mathrm{~m}, 2$ = Sturz $>1 \mathrm{~m}, 3=$ Sturz $>3 \mathrm{~m}, 4$ = Fahrrad, 5 = Fußball, 6 = Pferdesport, $7=$ sonstiger Sport, 8 = Toben/Spielen, 9 = Pkw-/Businsasse, 10 = Verkehrsunfall Fußgänger(in) gegen Pkw/Motorrad, $11=$ Verkehrsunfall Fahrradfahrer(in) gegen Pkw/Motorrad, $12=$ tätliche Auseinandersetzung/Gewalt, 13 = „umgeknickt“/Distorsion, 14 = Anpralltrauma, 15 = Quetschtrauma inkl. Gegenstände/Personen, die auf Patient(inn)en gefallen sind, 16 = kein Trauma beobachtet/erinnert, 17 = Sonstiges

Bei 99 Patient(inn)en konnte erfasst werden, wie sie verunfallt waren (siehe Abbildung 27, für die Erläuterung der Kategorien siehe Tabelle 3, Kapitel 2.2). Die meisten Patien$\mathrm{t}$ (inn)en waren aus einer Höhe von $\leq 1 \mathrm{~m}$ gestürzt, sie machten $11,11 \%$ des Patient(inn)enkollektivs (11/99) aus. Elf weitere Patient(inn)en waren ebenfalls gestürzt: sieben $(7,07 \%)$ von ihnen aus einer Höhe von einem Meter bis drei Metern und vier $(4,04 \%)$ aus einer Höhe von mehr als drei Metern. Insgesamt war mehr als ein Fünftel (22,22\% bzw. 22/99) der Frakturen durch Stürze verursacht.

Zweithäufigste Unfallursache waren „Toben/Spielen“ und „,umgeknickt'/Distorsion“ mit jeweils 10,01\% (10/99). Fast ebenso häufig, in 9,09\% (9/99) der Fälle, zogen sich Patient(inn)en eine Fraktur beim Fahrradfahren zu. Insgesamt macht Sport (Kategorien 4 bis 7) mehr als ein Fünftel (21,21\% bzw. 21/99) der Unfallursachen aus. Weitere 
Sportarten waren Fußball (6.06\% bzw. 6/99), Pferdesport (2,02\% bzw. 2/99) und sonstiger Sport (4,04\% bzw. 4/99).

16 Patient(inn)en waren im Straßenverkehr verunfallt (16,16\%). Die meisten von ihnen waren als Pkw- oder Businsasse in einen Unfall verwickelt: 8,08\% (8/99). Sechs Patient(inn)en (6,06\%) waren als Fußgänger(innen) von einem Pkw erfasst worden und zwei (2,02\%) als Fahrradfahrer(innen) mit Pkws zusammengestoßen.

Erfreulicherweise kam es selten zu „tätliche[r] Auseinandersetzung/Gewalt“, nur ein(e) Patient(in) war davon betroffen (1,01\%).

Lediglich zwei Patient(inn)en erlitten durch ein Anpralltrauma eine Femurfraktur (2,02\%). Deutlich häufiger waren Patient(inn)en von einem Quetschtrauma betroffen: Sieben Patient(inn)en (7,07\%) frakturierten sich dabei den Oberschenkel. Dazu zählten Unfallhergänge, bei denen eine Person oder Gegenstände auf den Patienten/die Patientin gefallen waren.

In fünf Fällen war kein Trauma erinnerlich oder beobachtet worden. In fünf weiteren Fällen hatten sich Unfallhergänge ereignet, die keiner der vorgenannten Kategorien zugeordnet werden konnten, wie beispielsweise: „Rotationsbewegung bei Krankengymnastik bei Patient[in] mit West-Syndrom“ oder „beim Umlagern plötzlich Schmerzen bei Patient[in] mit Rett-Syndrom" (Originalwortlaut Patient(inn)enakte). Diese Fälle wurden in Kategorie 17 zusammengefasst und machen insgesamt 5,05\% der Unfallhergänge aus.

Die Datenanalyse ergab einen Zusammenhang zwischen Alter und Komplikationsrisiko. Es wurden drei Altersgruppen unterschieden (unter drei Jahre, drei bis achteinhalb Jahre und über acht Jahre). Diese Unterscheidung wurde der Beschreibung der Unfallhergänge zugrunde gelegt. Dabei konnte Folgendes beobachtet werden: Ein Viertel der unter Dreijährigen verunfallte beim „Toben/Spielen“ und 17\% bei einem Sturz aus einer Höhe von unter einem Meter. Von den Drei- bis Achteinhalbjährigen erlitten jeweils $17 \%$ ein Quetschtrauma oder verletzten sich beim Fahrradfahren. Bei den über achteinhalbjährigen Patient(inn)en verunfallten viele als Verkehrsteilnehmer. So waren die meisten (15\%) als Fußgänger(in) von einem Auto erfasst worden. Jeweils weitere $12 \%$ waren als Pkw- oder Businsasse an einem Verkehrsunfall beteiligt und verletzt worden oder bei einem Sturz aus unter einem Meter Höhe. (Siehe auch Tabelle 16, Tabelle 17 und Tabelle 18 im Anhang).

Den 99 Patient(inn)en konnte ihre jeweilige Frakturart zugeordnet werden. So hatte die Hälfte derer, die sich im Rahmen eine Distorsion eine Fraktur zugezogen hatten, eine Spiralfraktur erlitten $(n=5)$. Ebenfalls an einer Spiralfraktur erkrankten $57 \%$ der 
Patient(inn)en mit einem Quetschtrauma ( $n=4)$. Die beiden Patient(inn)en, die beim Pferdesport verunfallten, frakturierten sich ihr Bein quer $(n=2)$. Zwei Drittel der Patient(inn)en, die sich beim Fußballspielen verletzten, erlitten eine Spiralfraktur $(n=4)$. Die Mehrzahl der Patient(inn)en, die aus einer Höhe von unter einem Meter gestürzt waren, zogen sich entweder eine Schräg- (27\%) oder eine Spiralfraktur (36\%) zu. Im Anhang (Tabelle 19 und Tabelle 20) ist eine ausführliche Auflistung der Unfallhergänge und jeweiligen Frakturtypen zusammengestellt.

In 13,4\% der Fälle (13/97) waren Frakturen pathologisch, ihnen war kein adäquates Trauma vorausgegangen. Außerdem gab es fünf Fälle, bei denen es sich eventuell um pathologische Frakturen handelte, die jedoch nicht als solche diagnostiziert worden waren (oder als „Verdacht auf pathologische Fraktur"). Die große Mehrheit, 81,46\% der Patient(inn)en (79/97), war von nicht pathologischen Frakturen betroffen (siehe Abbildung 28).

Es wurde festgestellt werden, dass 23,76\% der Untersuchten (24/101) neben Femurfrakturen unter weiteren Krankheiten litten. Die Begleiterkrankungen waren sehr heterogen, bspw. „Osteogenesis imperfecta“, „Infekt oberer Atemwege“, „Nierenarterienstenose“ oder „fibröse Dysplasie“. Abbildung 29 stellt die Ergebnisse grafisch dar. 


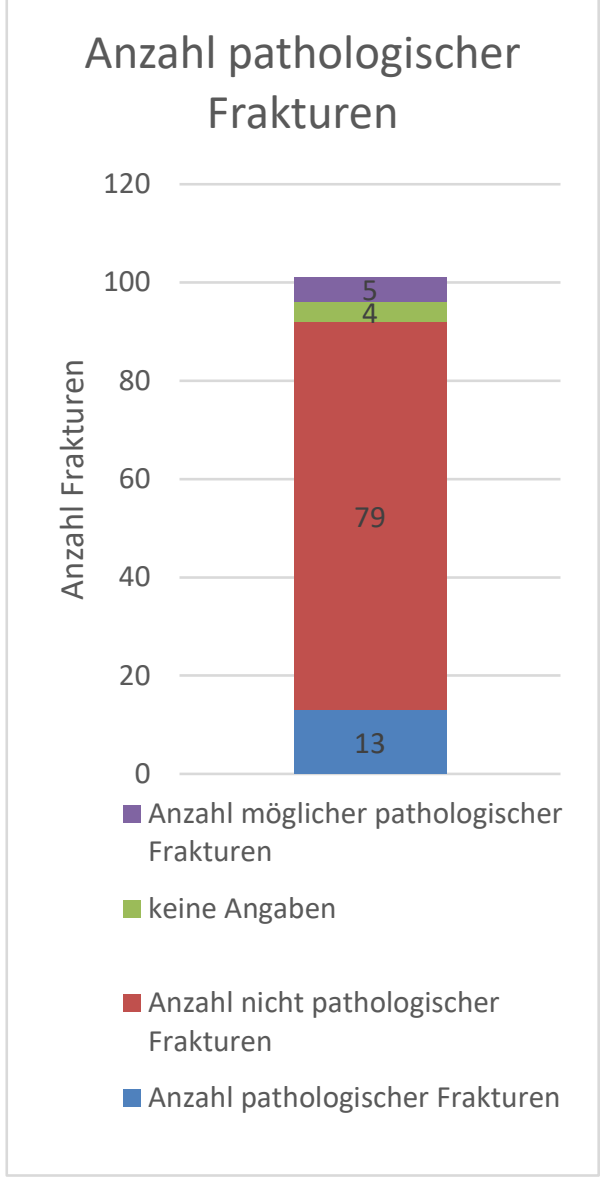

Abbildung 28: Häufigkeit von pathologischen Frakturen im Patient(inn)enkollektiv

\section{Begleiterkrankungen}

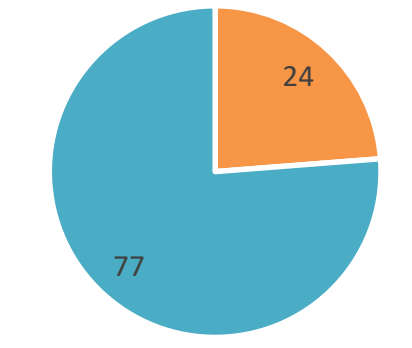

- Patienten mit Begleiterkrankung

- Patienten ohne Begleiterkrankung

Abbildung 29: Häufigkeit von Begleiterkrankungen im Patient(inn)enkollektiv

\subsection{Therapie}

Die Reposition der Brüche wurde operativ geschlossen, operativ offen oder im Rahmen einer konservativen Behandlung erreicht (in vier der Fälle gab es keine Angabe zur Art der Reposition). Bei 63,92\% (62/97) waren Frakturen operativ geschlossen reponiert worden. In 15 von 97 Fällen (15.46\%) war operiert, jedoch offen reponiert worden. Die konservative Behandlungsmethode mit Gipslonguette oder Overhead-Extension wurde in 19,58\% der Fälle (19/97) angewandt. Ein Patient war mit einer „Knochenkürettage, Knochentransplantation" behandelt worden. Er stellte jedoch mit 1,01\% eine Ausnahme dar (Abbildung 30). Eine Übersicht zur jeweiligen Versorgung der Frakturtypen findet sich im Anhang (Tabelle 23). 


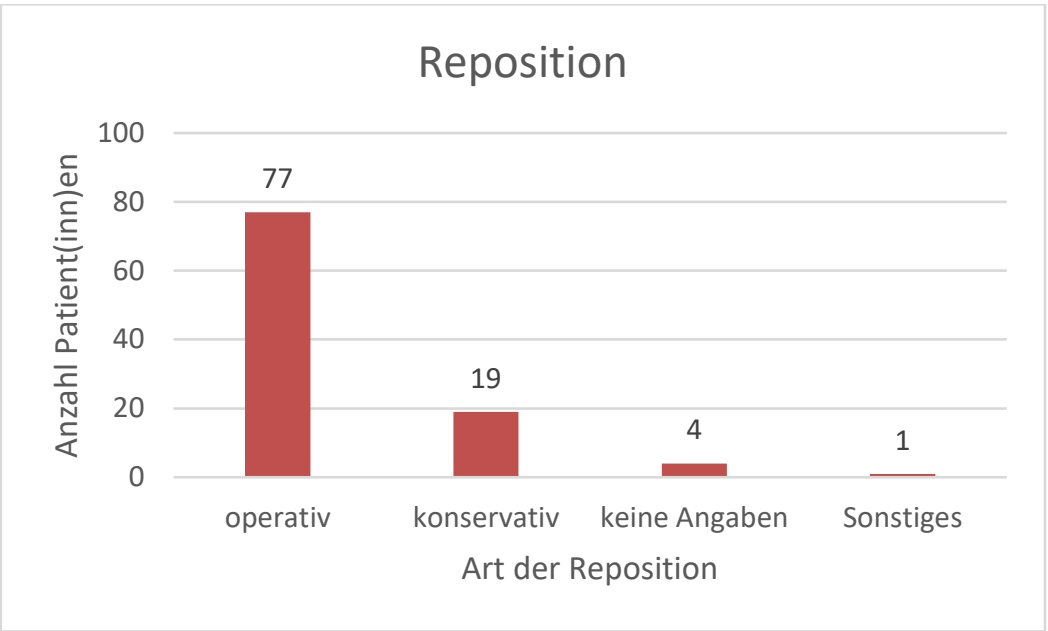

Abbildung 30: Arten der Reposition

Die verschiedenen konservativen Behandlungsmethoden waren jeweils gleich häufig angewandt worden: 42,10\% der Patient(inn)en (8/19) erhielten eine Gipslonguette, $36,84 \%$ von ihnen (7/19) die Overhead-Extensions-Therapie. Bei 21,05 \% (4/19) fehlen die Informationen zur Behandlungsmethode.

Bei der operativen Reposition wurden in $75,61 \%$ der Fälle (62/82) elastisch stabile intramedulläre Nägel (ESINs) benutzt. Sie waren das am häufigsten verwendete Repositionsmaterial. Elfmal war eine Platte auf dem Knochen angebracht worden, dies entspricht 13,42\%. In jeweils 3,66\% der Fälle (3/82) waren Kirschner-Drähte oder Fixateur externe verwendet worden. In jeweils nur einem Fall nutzte man einen Marknagel oder eine Hohl-Schraube zur Reposition. Eine grafische Darstellung der Daten zeigt Abbildung 31. Darüber hinaus zeigt Tabelle $21 \mathrm{im}$ Anhang, bei welchem Frakturtyp welches Material verwendet wurde.

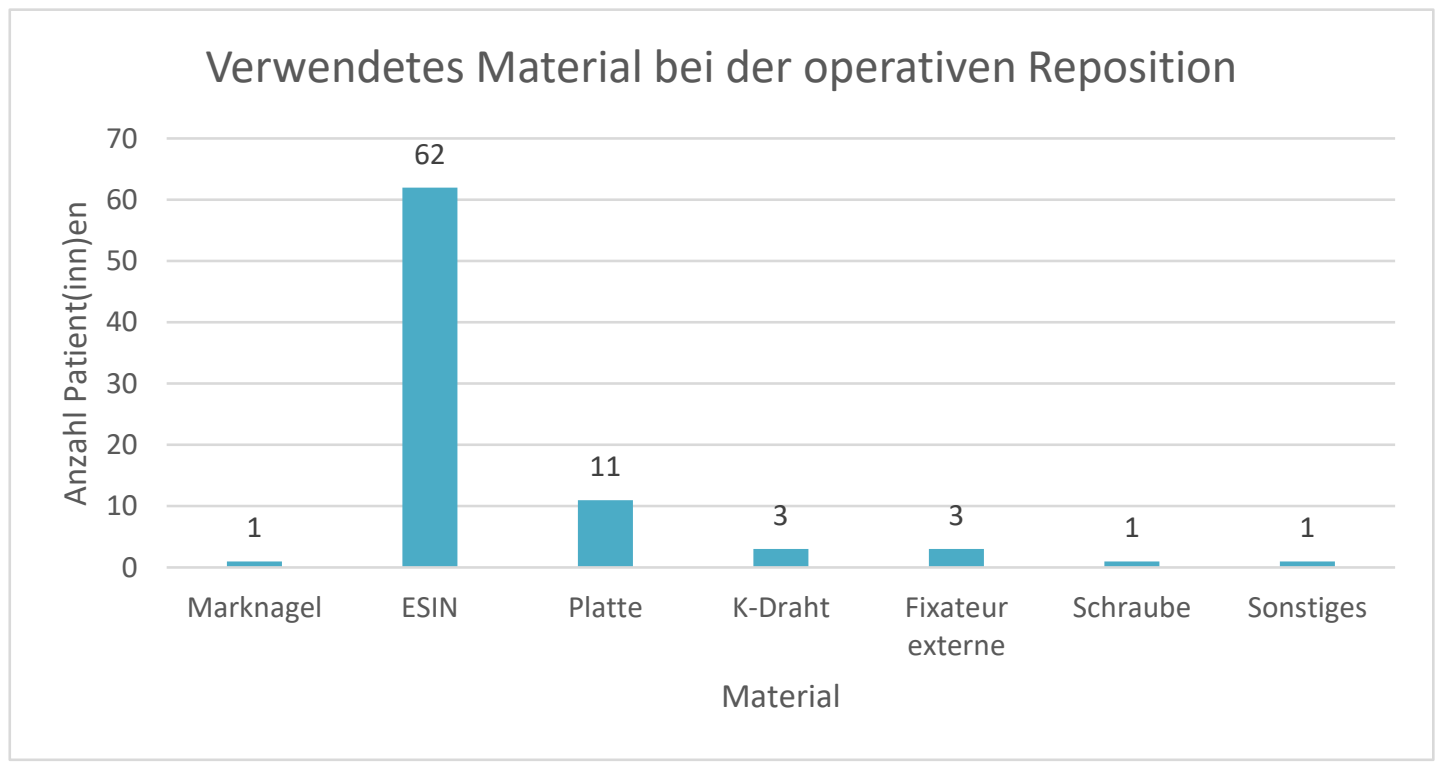

Abbildung 31: Verwendetes Material bei der Reposition im Falle einer Operation 
ANZAHL DER PATIENT(INN)EN PRO JEWEILIGER LIEGEDAUER

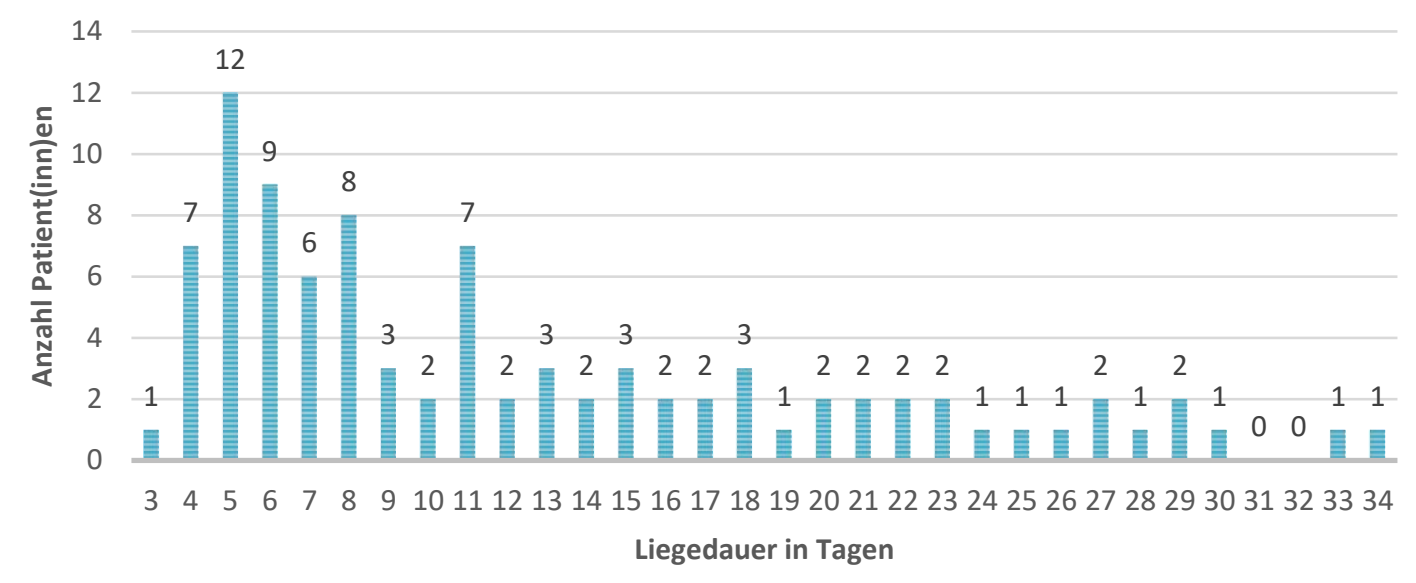

Abbildung 32: Verweildauer der Patient(inn)en im Krankenhaus

Bei 92 Patient(inn)en konnte die Dauer des Krankenhausaufenthaltes ermittelt werden: Sie waren im Durchschnitt 12,32 Tage im Krankenhaus. Die längste Liegedauer betrug 34 Tage, die kürzeste drei Tage. Die längsten Beobachtungen wurden nicht verwendet, da es sich höchst wahrscheinlich um „Ausreißer“ handelt (da sie die mittlere Liegedauer um das Fünffache überschritten).

Von 54 Patient(inn)en, denen ESINs eingesetzt worden waren, konnten Röntgenbilder ausgewertet und so der Durchmesser des Markraums ermittelt werden. Um Messfehler so gering wie möglich zu halten, war die Markhöhle sowohl auf der anterior-posterior sowie auf der lateralen Röntgenaufnahme ausgemessen und ein Mittelwert errechnet worden. Die Messungen wurden jeweils proximal und distal vom Frakturspalt durchgeführt.

Der Mittelwert für den Durchmesser des Markraums proximal der Fraktur betrug $9 \mathrm{~mm}$ bei einer Mess-Bandbreite von 4,3 $\mathrm{mm}$ bis $21 \mathrm{~mm}$. Ein Drittel der Patient(inn)en (18/54) lag knapp unter dem Durchschnittswert bei einem Durchmesser von 6 bis $8 \mathrm{~mm}$. Sie waren anteilig die größte Gruppe im Kollektiv. Abbildung 33 stellt die Mess-Ergebnisse dar. Lediglich ein Messwert lag außerhalb dieses Bereiches bei $51,55 \mathrm{~mm}$ und wurde als „Ausreißer" gewertet.

Distal der Fraktur war die Markhöhle durchschnittlich 9,15 mm breit. Der kleinste gemessene Durchmesser betrug 3,85 $\mathrm{mm}$ und der weiteste $30,6 \mathrm{~mm}$. Am häufigsten war auch hier die Kategorie "6 bis 8 mm", in 17 von 54 Fällen (31,48\%). Eine detaillierte Darstellung der Ergebnisse zeigt Abbildung 34. 


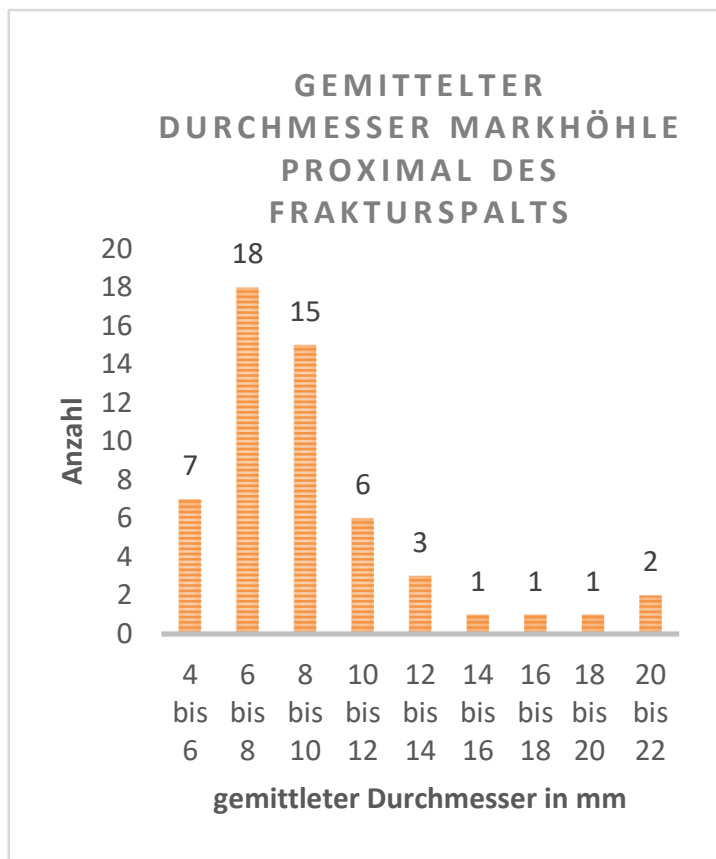

Abbildung 33: Durchmesser der Markhöhle proximal des Frakturspalts

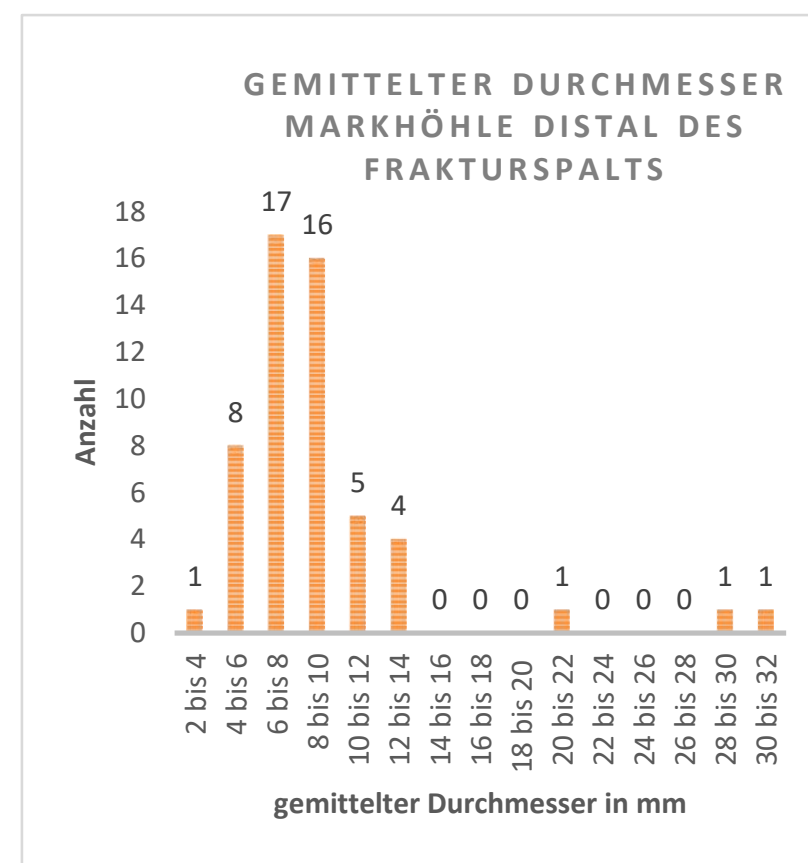

Abbildung 34: Durchmesser der Markhöhle distal des Frakturspalts

Ein weiterer Mittelwert wurde jeweils aus den proximalen und distalen Werten erstellt. Dieser erstellte Mittelwert wurde zur Dicke der eingesetzten ESINs ins Verhältnis gesetzt (Mittelwert proximal und distaler Durchmesser dividiert durch Dicke der ESINs). Abbildung 35 verdeutlicht die Ergebnisse von 48 Patient(inn)en.

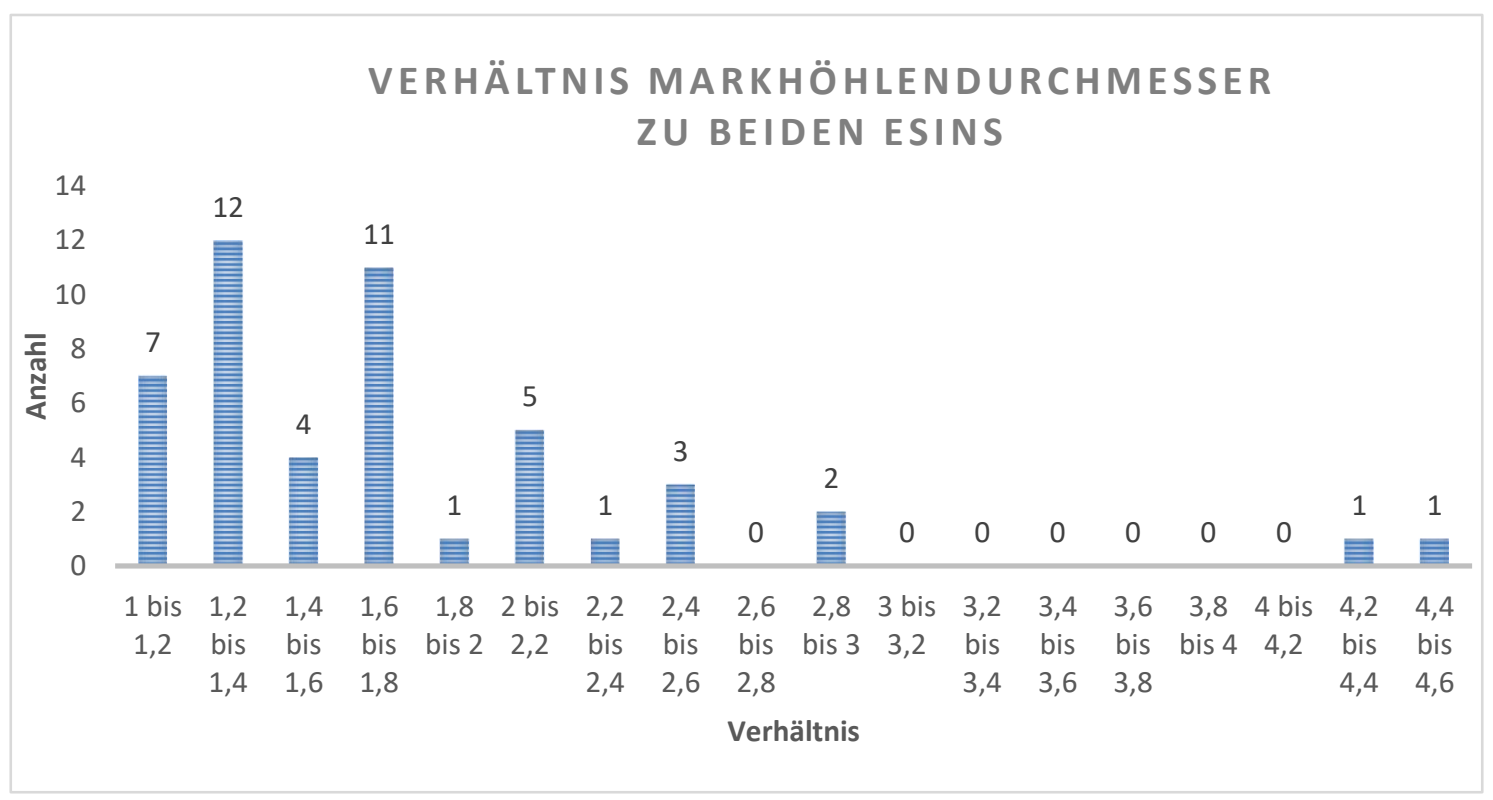

Abbildung 35: Verhältnis Markhöhlendurchmesser zur Dicke der elastisch stabilen Nägel

Am häufigsten traten die Verhältnisse der Gruppe „1,2 bis 1,4“ auf (25\%). Hierfür ein Beispiel eines Patienten/einer Patientin mit dem Verhältnis 1,2: Um den Markraum 
vollständig auszufüllen, würden die gewählten ESINs plus ein Fünftel ihrer Dicke benötigt. Das bedeutet auch, dass nach der Implantation der ESINs noch ungefüllter Markraum vorhanden war, genau „0,2 ESINs“.

Fast ebenso häufig waren, bezogen auf den Markraumdurchmesser, dünnere ESINs in den Knochen eingesetzt worden, sodass bei elf von 48 Patient(inn)en (22,91\%) ein Verhältnis von Markraumdurchmesser zu ESINs von „1,6 bis 1,8“ ermittelt wurde.

Der kleinste Quotient (damit dickste ESINs im Verhältnis zur Markhöhle) betrug 1 und kam bei zwei Patient(inn)en vor. Der größte Quotient belief sich auf 4,52 und lag bei nur einem Patienten bzw. einer Patientin vor. Der Durchschnittswert ergab ein Verhältnis von 1,77. Bei diesen Patient(inn)en füllten die ESINs mehr als die Hälfte des Markraums aus.

Abbildung 36 zeigt die Häufigkeit der jeweiligen Wochenabstände zwischen erster Operation zum Einsetzen von Kraftträgern und der zweiten Operation zur Entfernung des Metalls. In 47 Fällen konnten Daten erhoben werden. Im Durchschnitt blieben die Implantate 44 Wochen lang im Körper. Sechs Patient(inn)en (12,77\%) ließen sich das Metall nach acht Wochen entfernen, es war der am häufigsten gewählte Zeitpunkt. Der zweithäufigste Zeitpunkt war nach elf Wochen (in 8,51\% der Fälle). Einem Patienten bzw. einer Patientin musste bereits nach wenigen Tagen der Kraftträger wieder entfernt werden. Der längste Zeitraum bis zur Implantatentfernung betrug mehr als vier Jahre (229 Wochen).

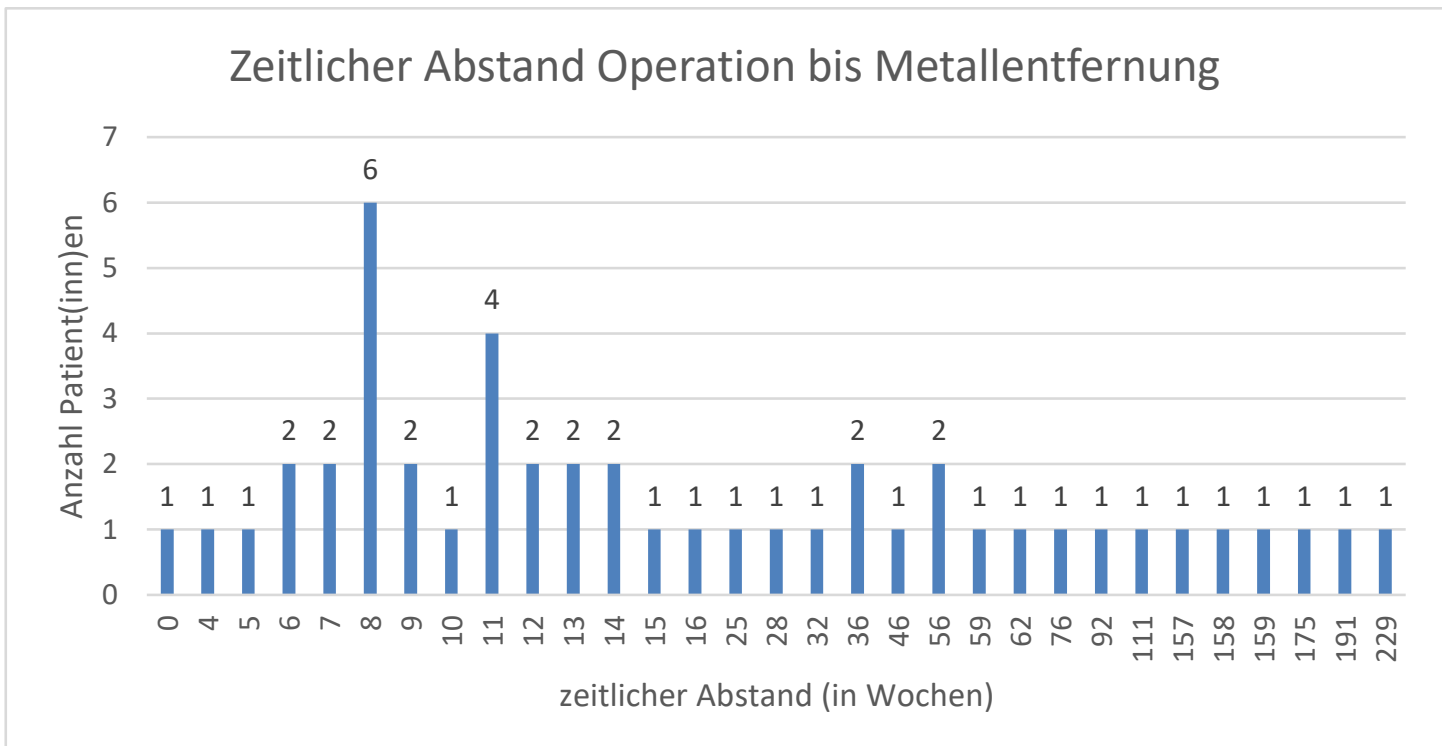

Abbildung 36: Zeitlicher Abstand zwischen Operation und Metallentfernung (in Wochen) 


\subsection{Komplikationen: intra- und postoperative Komplikationen, Komplikationsformen, mögliche Einflussfaktoren, Verfahrens- wechsel, Fehlstellung nach Böhm}

Bei 46,54\% der Patient(inn)en (47/101) traten keine Komplikationen während der Operation auf, bei 33,66\% (34/101) lagen keine Angaben vor, bei 18,81\% (19/101) hatte keine Operation stattgefunden. In einem Fall war das Implantat-Material fehlerhaft, sodass bei einem Repositionsmanöver die endständige Kugel eines elastisch intramedullären Nagels abbrach und eine frühe Metallentfernung nach sechs Wochen angesetzt wurde. Mit 0,99\% (1/101) war die intraoperative Komplikation also eine Ausnahme (siehe Abbildung 37).

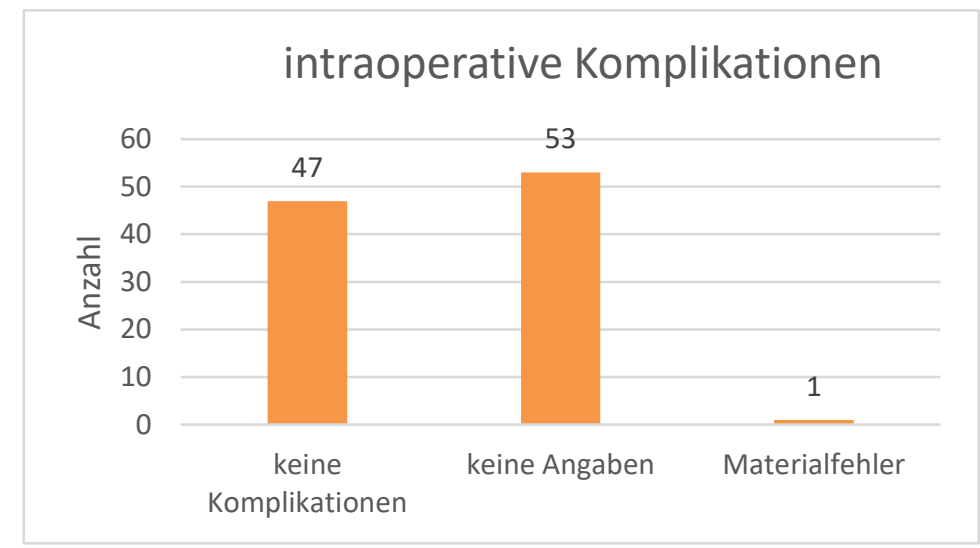

Abbildung 37: Häufigkeit von intraoperativen Komplikationen

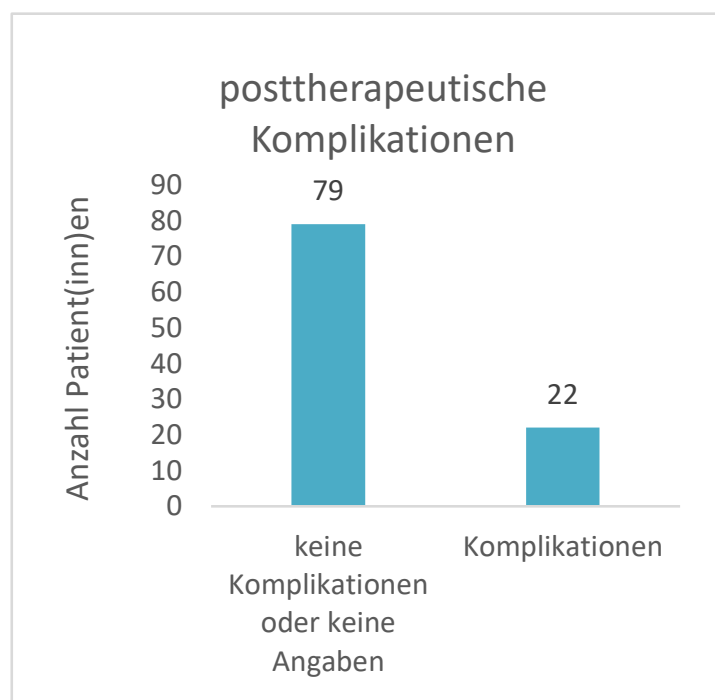

Abbildung 38: Häufigkeit der posttherapeutischen Komplikationen

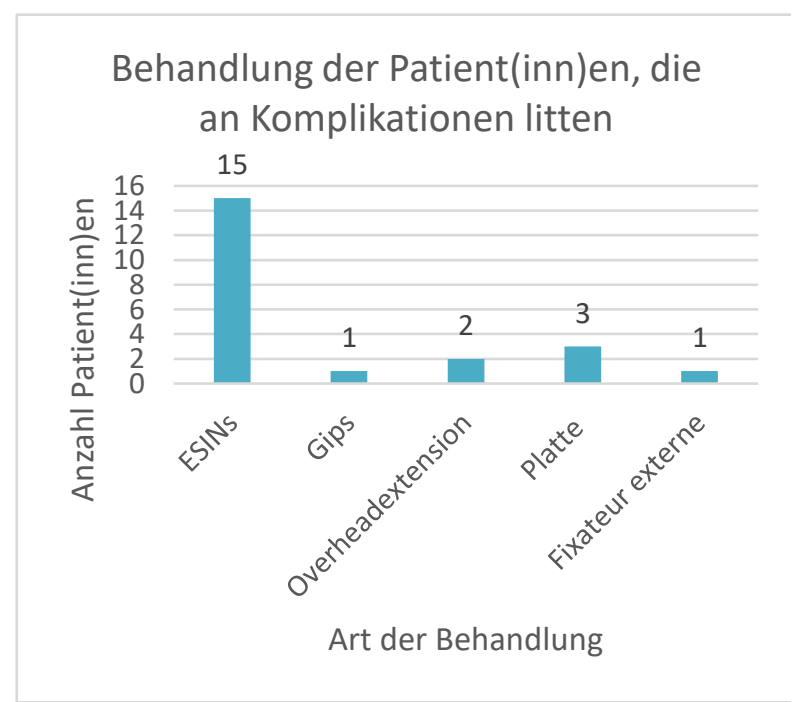

Abbildung 39: Behandlung der Patient(inn)en, die Komplikationen angaben

Über Beschwerden nach der Therapie berichteten 22 der Behandelten (21,78\%). Mehr als der Hälfte von ihnen (68,12\%) waren ESINs eingesetzt worden. Die übrigen sieben 
Patient(inn)en waren konservativ behandelt oder operiert worden (siehe Abbildung 39). Abbildung 38 verdeutlicht, dass die Femurschaftfraktur dennoch zu den komplikationsarmen Erkrankungen zählt: 78,22\% der Patient(inn)en (79/101) hatten einen komplikationslosen Therapieverlauf.

Die verschiedenen Komplikationstypen und ihre jeweilige Häufigkeit sind Abbildung 40 zu entnehmen. Am häufigsten, in acht von 19 Fällen (42,11\%), waren die Patient(inn)en von Wachsstumsstörungen betroffen. In vier Fällen war es zu einem überschießenden Wachstum, einem „Overgrowth“, gekommen. In weiteren vier Fällen oder eventuell auch auf Grund von überschießendem Wachstum waren vier Patient(inn)en von unterschiedlicher Beinlänge betroffen. Unter der zweithäufigsten postoperativen Komplikation, der „passageren Einschränkung der Kniegelenksbeweglichkeit“ litten sieben Patient(inn)en (7/19 bzw. 36,84\%). Jeweils einoder zweimalig traten die Komplikationen „Hautperforation“, „hypertrophe Narbe“, "Malunion“, „sekundäre Dislokation“, „Malreduktion“, „Kompartmentsyndrom“, „Pin Tract Infection“ und „Wundheilungsstörung" auf.

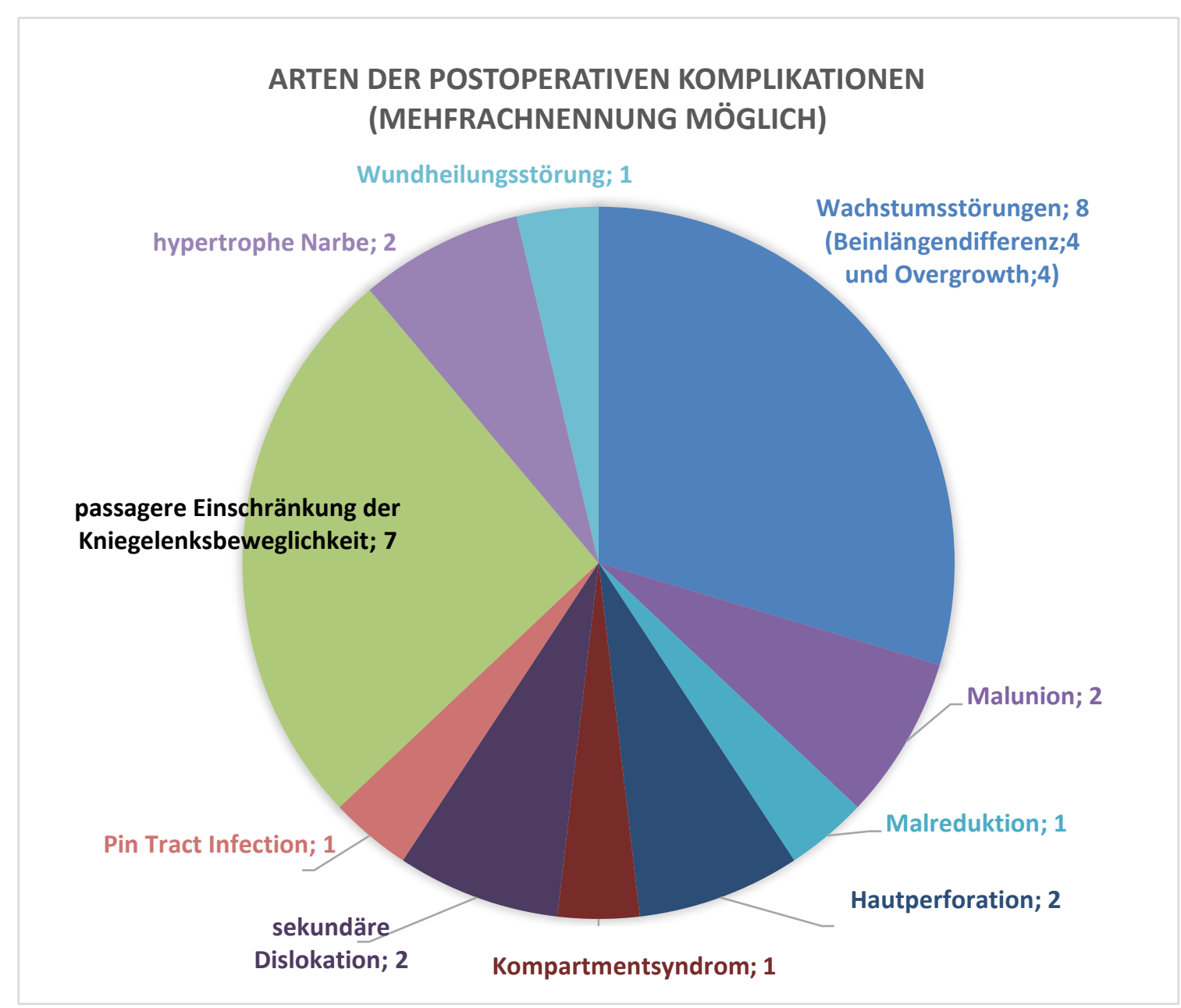

Abbildung 40: Arten der postoperativen Komplikationen (Mehrfachnennungen möglich) 
In fünf Fällen kam es infolge von Komplikationen zum Verfahrenswechsel. Darunter waren zwei Patient(inn)en mit Fixateur externe, bei denen jeweils zum Platten- bzw. ESIN-Verfahren gewechselt wurde. Bei zwei Patient(inn)en, die konservativ behandelt worden waren, wurden ebenfalls ESINs eingesetzt. Aber auch diese ESINs wurden in einem Fall ausgetauscht, dem Patienten/der Patientin wurde in der Folge eine Platte implantiert. Somit war die Revisionsrate der konservativen Therapieansätze (2/19 bzw. $10,53 \%)$ doppelt so hoch verglichen mit der bei operativer Therapie (4/77 bzw. 5,2\%). Dabei ist auffällig, dass zwei Drittel der mit Fixateur externe behandelten Kinder (66,67\%) re-operiert werden mussten. Die revisionspflichtigen Patien(inn)en, die mit ESINs behandelt wurden, machten lediglich 3,23\% (2/62) aus. Es bestehen also große Unterschiede bei der Re-Operationsrate innerhalb der operativen Verfahren.

Böhm (2007) beschreibt die bei Kindern tolerable Fehlstellung auch für diaphysäre Frakturen des Oberschenkels (vgl. 1.6.2). Im Patient(inn)enkollektiv war diese zweimal nicht erfüllt. Bei einem elfjährigen Patienten war nach der ersten operativen Stabilisierung der Fraktur mit ESINs eine Varusfehlstellung von $15^{\circ}$ festgestellt worden und nach der Re-Operation lag eine Verkürzung des Beines von $2,5 \mathrm{~cm}$ vor. Bei dem zweiten Patienten handelt es sich um einen Siebenjährigen, der nach der Plattenentfernung unter einer ad axim Fehlstellung nach dorsal litt.

\subsubsection{Einflussfaktor: Alter}

Das Alter der Patient(inn)en scheint für das Risiko postoperativer Komplikationen eine wesentliche Rolle zu spielen. Abbildung 41 zeigt, dass das Risiko von der Geburt bis zum fünften Lebensjahr kontinuierlich sinkt, danach wieder ansteigt, bis es um das zwölfte/dreizehnte Lebensjahr sein Maximum erreicht und danach wieder abnimmt. Diesen Zusammenhang kann man durch eine kubische Regression auch statistisch nachweisen (Siehe Tabelle 24, Spalte 2, Zeilen 5-7).

Des Weiteren verdeutlicht die Regression in Tabelle 24 (Spalte 1, Zeilen 1-4), dass das geringste Risiko für Patient(inn)en in der Altersgruppe drei- bis achteinhalb Jahre vorliegt. Sie haben ein um $28 \%$ signifikant $(P=0.015)$ niedrigeres Komplikationsrisiko als die unter Dreijährigen. Auf der anderen Seite haben Patient(inn)en zwischen zwölf bis dreizehn Jahren ein $26,5 \%$ signifikant $(P=0.066)$ höheres Risiko als die unter Dreijährigen ${ }^{3}$. Zusammenfassend zeigt sich, dass bis zu $20 \%$ der Variationen der

\footnotetext{
${ }^{3}$ In den weiteren Beobachtungen wird die Gruppe der 12- bis 13-Jährigen nicht als separate Gruppe aufgeführt, da die Anzahl der Patient(inn)en gering ist und die Aussagekraft der Ergebnisse nicht erhöht würde.
} 
Komplikationen allein durch die Variable Alter zu erklären sind (siehe Tabelle 24, Spalte 1, Zeile 10).

Diese Effekte sind robust für das Multi-Variablen-Modell mit der zusätzlichen Variable „Verhältnis Markraumdurchmesser zur ESIN-Drahtdicke = 1,5 - 2“. Somit ist die Signifikanz der Ergebnisse des vorherigen Tests bestätigt. Das bedeutet, dass das Alter signifikant ist, auch wenn man gleichzeitig im Modell für das Verhältnis Markraumdurchmesser zu Drahtdicke testet. Das heißt, für die Wahrscheinlichkeit, eine Komplikation zu erleiden, spielt nicht nur das Alter drei- bis achteinhalb Jahre und zwölf bis dreizehn Jahre, sondern auch das Verhältnis von Markraumdurchmesser zu Drahtdicke (1,5-2) eine signifikante Rolle. Diese Variablen zusammen erklären 30\% der Komplikationen (siehe Tabelle 25).

Vergleicht man die Altersgruppen miteinander, stehen die Chancen für Komplikationslosigkeit am besten für die Vier- bis Achtjährigen, wenn auch nicht signifikant (siehe Tabelle 26).

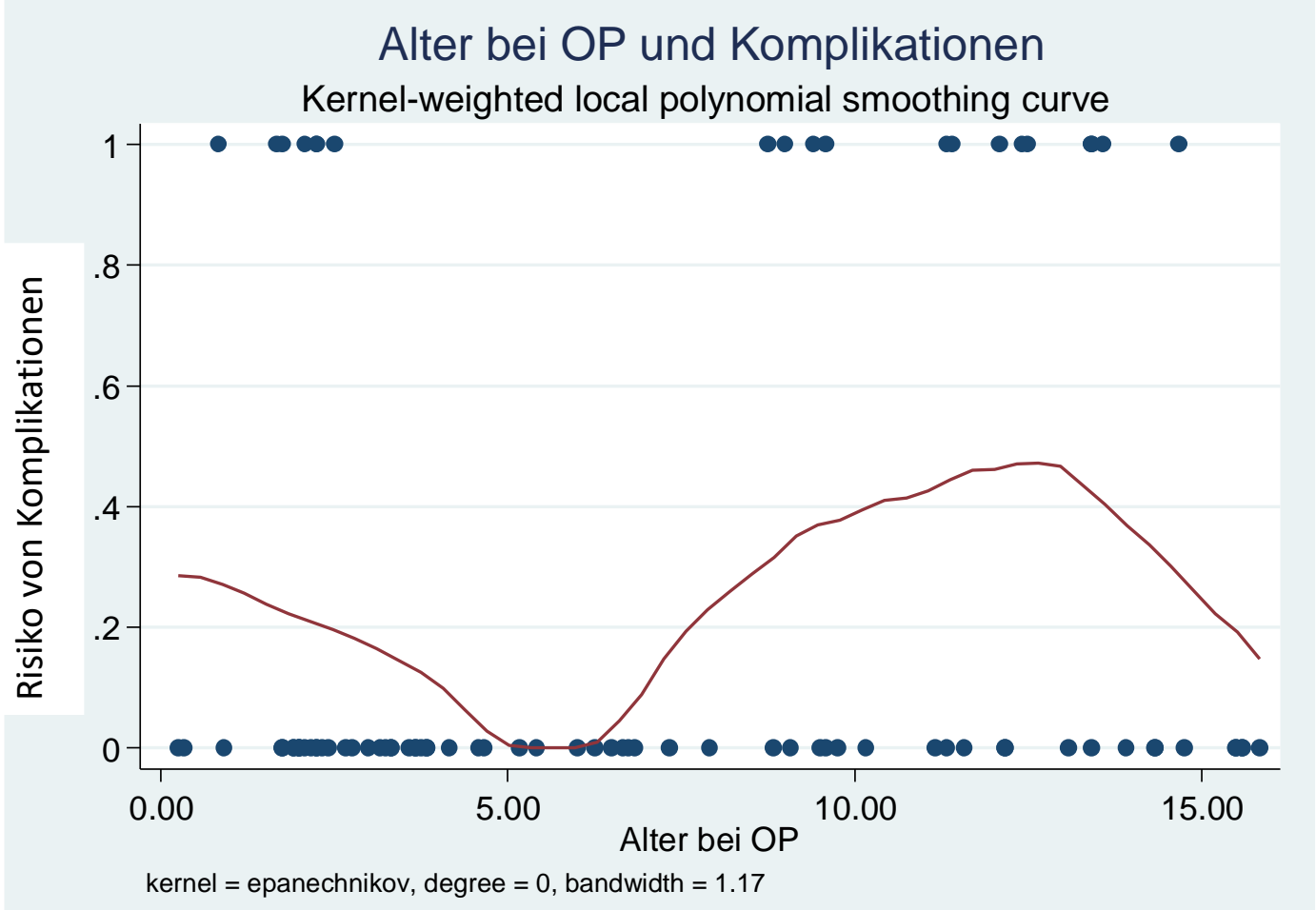

Abbildung 41: Komplikationswahrscheinlichkeit im jeweiligen Lebensjahr

Im Anschluss an dieses Ergebnis wurde analysiert, welche Frakturtypen sich die Patien$t$ (inn)en in den drei unterschiedenen Altersgruppen (unter drei, zwischen drei bis achteinhalb sowie über achteinhalb) zugezogen hatten. (Einteilung siehe 3.1)

Besonders häufig litten unter Dreijährige unter Spiralfrakturen, und zwar 44\%. Sie stellten fast ein Drittel der Patient(inn)en dar, die sich eine Spiralfraktur zugezogen hatten 
(11/32). Die zweithäufigste Fraktur in dieser Altersgruppe war die Schrägfraktur: fünf der Betroffenen zogen sich eine solche zu und machten damit ein Drittel aller Patien$t$ (inn)en aus, die unter einer Schrägfraktur litten (5/15).

Patient(inn)en zwischen drei und achteinhalb Jahren, also die Altersgruppe mit dem geringsten Komplikationsrisiko, erlitten ebenfalls hauptsächlich Spiralfrakturen (38\%). Jeweils viermal traten Quer-, Schräg- und „Femurschaftfrakturen“ auf. Auch die über achteinhalbjährigen Patient(inn)en erlitten am häufigsten Spiralfrakturen (23\%). Die dazugehörigen Übersichten finden sich im Anhang ( Tabelle 27, Tabelle und Tabelle 29).

\subsubsection{Einflussfaktoren: Body-Mass-Index, Perzentilkurven}

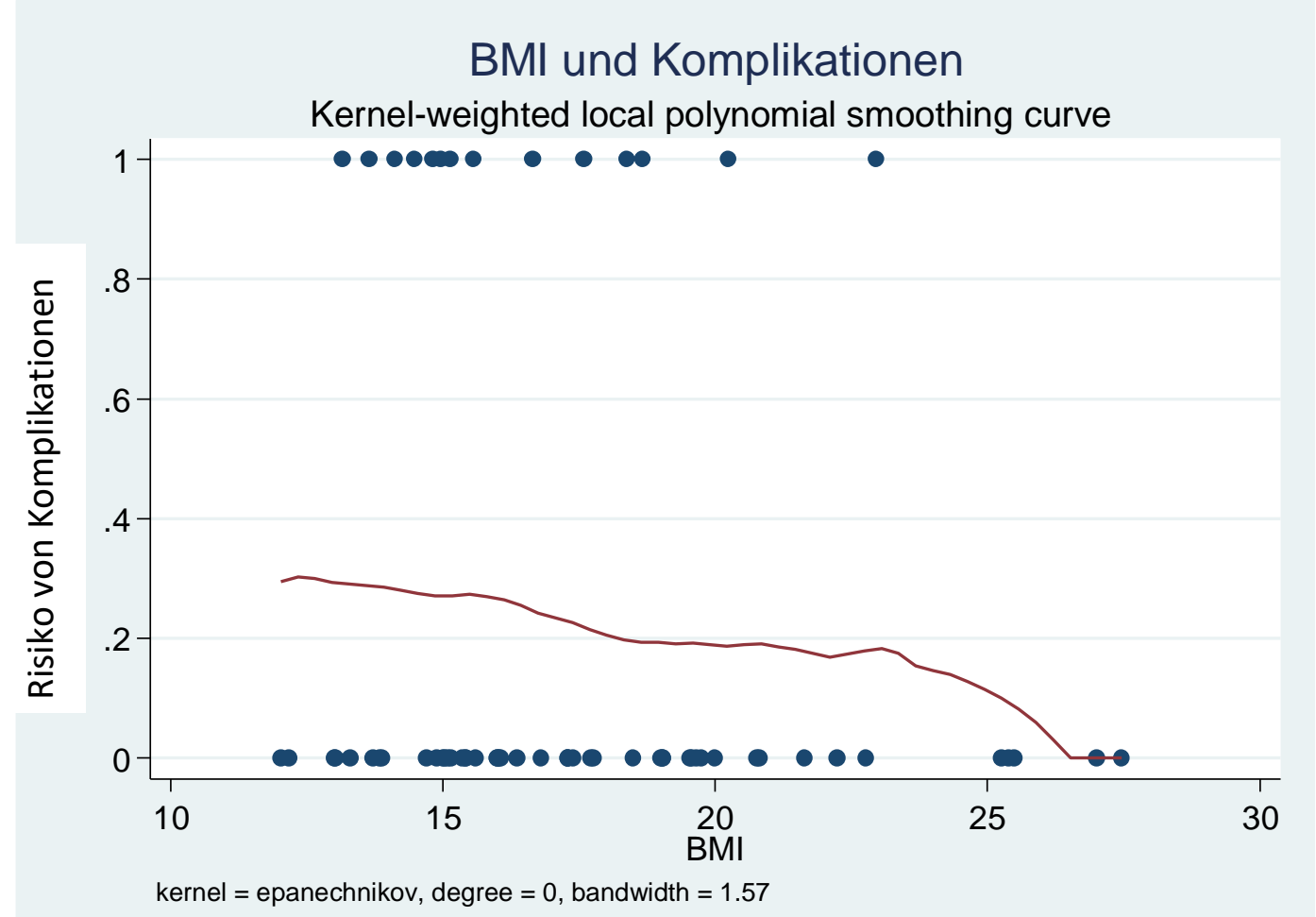

Abbildung 42: Einfluss des Body-Mass-Indexes auf die postoperative Komplikationsrate

Abbildung 42 stellt den Zusammenhang zwischen dem Body-Mass-Index und Komplikationen graphisch dar. Nach diesem Kernel Regressions Graphen scheint eine negative Korrelation von Body-Mass-Index und Komplikationen zu bestehen. Tabelle 30 (Spalte 1) zeigt aber, dass dieser Zusammenhang nicht statistisch signifikant ist. Hierbei muss beachtet werden, dass es nur wenige hohe Body-Mass-Index-Werte gab, die analysiert werden konnten. Des Weiteren ergab eine Regression von Alter und Body-Mass-Index Folgendes (Tabelle 30, Spalte 2): Mit jedem weiteren Lebensjahr der Patient(inn)en stieg der Body-Mass-Index signifikant um 3,5 (Koeffizient 0.351, P=0.004). Je höher das 
Lebensalter, desto höher war der Body-Mass-Index. Um den Zusammenhang zu kontrollieren, wurde ein Multi-Variablen-Modell erstellt mit der zusätzlichen Variable Alter (siehe Tabelle 30, Spalte 3). Die Kontrolle zeigte, dass ein hoher Body-Mass-Index nicht vor Komplikationen schützt, sondern nur mit höherem Alter korreliert ist, welches wohl das Komplikationsrisiko senkt.

Es gab keine signifikanten Testergebnisse für den Zusammenhang von Komplikationen und Perzentilkurven. Eine Ursache dafür könnte die relativ geringe Anzahl der Beobachtungen ( $n=63$ ) sein (siehe Tabelle $31 \mathrm{im}$ Anhang).

\subsubsection{Einflussfaktor auf Komplikationen: Frakturtyp}

Die Spiralfraktur war die häufigste Fraktur ( $n=32$ ) im Patient(inn)enkollektiv. Ein Viertel dieser Patient(inn)en litt unter Komplikationen (Mean =0,25). Die Spiralfraktur war damit die Frakturform mit den dritthäufigsten Komplikationen.

Die als „Femurschaftfraktur" kategorisierte Frakturform war als zweithäufigste Form zugleich die komplikationsreichste $(n=16$, Mean $=0,31)$.

Fast genauso häufig traten Quer- und Schrägfrakturen auf (jeweils $n=15$ ). Komplikationsreicher war die Querfraktur (Mean $=0,26$ ), außerdem stellte sie insgesamt die Frakturform mit den zweithäufigsten Komplikationen dar. Patient(inn)en mit Schrägfrakturen hingegen litten nur mit einer Wahrscheinlichkeit von 20\% unter Komplikationen (Mean =0,2).

Die Spiralfraktur als zahlenmäßig häufigste Frakturform weist ein geringeres Komplikationsrisiko auf als die deutlich selteneren Formen „Femurschaftfraktur", Quer- und Schrägfraktur (siehe Tabelle 7).

Tabelle 7: Häufigkeiten der verschiedenen Frakturtypen und die jeweilige Wahrscheinlichkeit, Komplikationen zu erleiden. Die Quer- und Femurschaftfraktur waren die komplikationsreichsten Brüche.

Frakturtypen: Querfraktur, Schrägfraktur, Spiralfraktur, Trümmerfraktur (sechs oder mehr Fragmentteile), Mehrfragmentfraktur (drei bis sechs Fragmentteile), Femurschaftfraktur, nicht zuzuordnen/ Sonstige

\begin{tabular}{r|rrr} 
& Summary of Komplikation post op \\
Frakturtyp & Mean & Std. Dev. & Freq. \\
\hline Querfrakt & .26666667 & .45773771 & 15 \\
Schrägfra & .2 & .41403934 & 15 \\
Spiralfra & .25 & .43994135 & 32 \\
Trümmerfr & 0 & 0 & 1 \\
nur Mehrf & .14285714 & .37796447 & 7 \\
nur Femur & .3125 & .47871355 & 16 \\
nicht zuz & .06666667 & .25819889 & 15 \\
\hline Total & .21782178 & .41482431 & 101
\end{tabular}




\subsubsection{Einflussfaktoren: Art der Versorgung, Repositionsform}

Bezüglich der Art der Reposition konnte mittels eines t-Tests gezeigt werden, dass eine konservative Therapie gegenüber einer operativen Versorgung mit signifikant $(P=0.04)$ weniger Komplikationen einhergeht. So litten konservativ behandelte Kinder mit einer mittleren Wahrscheinlichkeit von 15\% unter Komplikationen, hingegen 23\% der Kinder, die mit einer der anderen Behandlungsmethoden (Operation und Reposition offen, Operation und Reposition geschlossen, Operation sonstige) versorgt worden waren. Weitere Details finden sich in Tabelle 8 und im Anhang in Tabelle 32.

Tabelle 8: Wahrscheinlichkeit für Komplikationen bei konservativer Behandlung (1) gegenüber den anderen Behandlungsarten (0)

\begin{tabular}{r|rrr}
$\begin{array}{c}\text { Operation_k } \\
\text { onservativ }\end{array}$ & $\begin{array}{r}\text { Summary of Komplikation post op } \\
\text { Mean }\end{array}$ & Std. Dev. & Freq. \\
\hline 0 & .23170732 & .4245195 & 82 \\
1 & .15789474 & .37463432 & 19 \\
\hline Total & .21782178 & .41482431 & 101
\end{tabular}

Das durchschnittliche Komplikationsrisiko nach offener Operation lag bei 33\% und ist signifikant. Im Vergleich dazu lag die Komplikationswahrscheinlichkeit der anderen Therapien bei 20\% (siehe Tabelle 9 und im Anhang Tabelle 33).

Tabelle 9: Wahrscheinlichkeit für Komplikationen bei offener operativer Behandlung (1) im Vergleich zu allen anderen Therapiemethoden (0)

\begin{tabular}{r|rrr}
$\begin{array}{r}\text { Operation_o } \\
\text { ffen }\end{array}$ & $\begin{array}{r}\text { Summary of } \\
\text { Mean }\end{array}$ & Std. Dev. & Freq. \\
\hline 0 & .19767442 & .40058097 & 86 \\
1 & .33333333 & .48795004 & 15 \\
\hline Total & .21782178 & .41482431 & 101
\end{tabular}

Auch im direkten Vergleich mit geschlossenen Operationen scheint die offene Operation die ungünstigere zu sein. So hatten Patient(inn)en, die offen operiert wurden, ein mit 12\% signifikant höheres Risiko (P-Wert $=0,01$ ) unter Komplikationen zu leiden, als Patient(inn)en, die geschlossen operativ versorgt wurden (Mean =0,21). Siehe Tabelle 10 und t-Test im Anhang Tabelle 34. 
Tabelle 10: Mittlere Wahrscheinlichkeit Komplikationen zu erleiden nach einer offenen Operation (1) im Vergleich zur geschlossenen Operation (0)

\begin{tabular}{r|rrr}
$\begin{array}{r}\text { Operation_o } \\
\text { ffen }\end{array}$ & $\begin{array}{r}\text { Summary of Komplikation post OP } \\
\text { Mean }\end{array}$ & Std. Dev. & Freq. \\
\hline 0 & .20967742 & .41040151 & 62 \\
1 & .33333333 & .48795004 & 15 \\
\hline Total & .23376623 & .42600049 & 77
\end{tabular}

\subsubsection{Einflussfaktoren: Reposition und Frakturform, Material und Frakturform}

Ausgehend von der Fragestellung, ob sich bei bestimmten Frakturtypen eine bestimmte Repositionsart empfiehlt um das Komplikationsrisiko gering zu halten, ist der Zusammenhang dieser möglichen Einflussfaktoren untersucht worden. In Tabelle 11 sind die Repositionsformen der jeweiligen Frakturtypen aufgelistet.

Bezogen auf das Kollektiv der geschlossen Versorgten ( $n=62)$, stellen die Patient(inn)en mit Spiralfraktur die zahlenmäßig größte Gruppe dar (22/62). Betrachtet man ausschließlich die Patient(inn)en mit Spiralfrakturen, so waren $71 \%$ geschlossen versorgt worden. Höher lag der prozentuale Anteil der geschlossen operativ versorgten Patient(inn)en in der Kategorie „Femurschaftfraktur“ mit 85\% (11/13).

Die konservative Therapie wurde zumeist bei Spiralfrakturen angewandt oder bei Frakturen, die keiner der Frakturkategorien eindeutig zuzuordnen waren (jeweils siebenmal, entspricht 23\% aller Spiralfrakturen und 47\% aller Frakturen der Kategorie 7). Offen operiert wurde bei $71 \%$ der Mehrfragmentfrakturen und einem Drittel der Querfrakturen (jeweils fünfmal). 
Tabelle 11: Reposition bei den jeweiligen Frakturtypen (Daten von 97 Patient(inn)en), Frakturtypen: 1 = Querfraktur, 2 = Schrägfraktur, 3 = Spiralfraktur, $4=$ Trümmerfraktur, 5 = Mehrfragmentfraktur, 6 = Femurschaftfraktur, 7 = nicht zuzuordnen

\begin{tabular}{|c|c|c|c|c|c|}
\hline $\begin{array}{r}\text { Frakturtyp } \\
\text { (1=Querfra } \\
\text { ktur, } \\
2=\text { Schrägfr } \\
\text { aktur, } \\
\text { 3=Spiralfr } \\
\text { aktur } \\
\text { 4=Trümmerf } \\
\text { raktur, } 5\end{array}$ & Sonstiges & $\begin{array}{r}\text { Versorgung } \\
\text { gesch } \\
\text { geschloss }\end{array}$ & $\begin{array}{l}\text { initial of } \\
\text { lossen } \\
\text { konservat }\end{array}$ & $\begin{array}{l}\text { oder } \\
\text { offen }\end{array}$ & Total \\
\hline \multirow[t]{2}{*}{1} & 0 & 9 & 1 & 5 & \\
\hline & 0.00 & 60.00 & 6.67 & 33.33 & 100.00 \\
\hline \multirow[t]{2}{*}{2} & 0 & 10 & 2 & 3 & \\
\hline & 0.00 & 66.67 & 13.33 & 20.00 & 100.00 \\
\hline \multirow[t]{2}{*}{3} & 0 & 22 & 7 & 2 & \\
\hline & 0.00 & 70.97 & 22.58 & 6.45 & 100.00 \\
\hline \multirow[t]{2}{*}{4} & 0 & 1 & 0 & 0 & 1 \\
\hline & 0.00 & 100.00 & 0.00 & 0.00 & 100.00 \\
\hline \multirow[t]{2}{*}{5} & 0 & 2 & 0 & 5 & 7 \\
\hline & 0.00 & 28.57 & 0.00 & 71.43 & 100.00 \\
\hline \multirow[t]{2}{*}{6} & 0 & 11 & 2 & 0 & \\
\hline & 0.00 & 84.62 & 15.38 & 0.00 & 100.00 \\
\hline \multirow[t]{2}{*}{7} & 1 & 7 & 7 & 0 & \\
\hline & 6.67 & 46.67 & 46.67 & 0.00 & 100.00 \\
\hline \multirow[t]{2}{*}{ Total } & 1 & 62 & 19 & 15 & 97 \\
\hline & 1.03 & 63.92 & 19.59 & 15.46 & 100.00 \\
\hline
\end{tabular}

Eine detaillierte Angabe, bei welchem Frakturtyp das jeweilige Material verwendet wurde, findet sich im Anhang in Tabelle 21. Am häufigsten waren ESINs bei Spiralfrakturen (22mal), Querfrakturen (zehnmal) und Schrägfrakturen (zehnmal) eingesetzt worden. Die Implantation einer Platte erfolgte bei Quer- (viermal), Spiral- (dreimal), Mehrfragment- (zweimal) und Schrägfrakturen (zweimal). Die konservative OverheadExtensions-Therapie war insgesamt achtmal angewandt worden, allein fünfmal bei einem Patienten bzw. einer Patientin mit einer Spiralfraktur. Dies sind die Ergebnisse einer Analyse von insgesamt 98 Patient(inn)en.

Es wurde überprüft, ob Kombinationen von Frakturtypen und intraoperativem Material vorlagen, die das Komplikationsrisiko erhöhten. Die Ergebnisse waren jedoch nicht signifikant: Es gab weder eine Frakturform, die die Komplikationswahrscheinlichkeit mit den jeweils gewählten Therapien erhöhte, noch Implantationsmaterial, das den Behandlungserfolg signifikant beeinflusste (siehe Anhang Tabelle 35 und Tabelle 36). 


\subsubsection{Einflussfaktor: Verhältnis Markraumdurchmesser zu ESIN-Drahtdicke}

Der Graph in Abbildung 43 veranschaulicht das Verhältnis von Markraumdurchmesser zu Drahtdicke und das resultierende Komplikationsrisiko. Es scheint, dass das Komplikationsrisiko bei einem Verhältnis = 1 unter $20 \%$ liegt, danach zunimmt bis es seinen Höhepunkt bei etwa 1,6 erreicht. Daraufhin fällt das Komplikationsrisiko sogar unter den Anfangswert. In der logistischen Regression (siehe Tabelle 37, Zeile 2) zeigt sich, dass dieser Zusammenhang auch statistisch signifikant ist. Konkret bedeutet dies:

Liegt das Verhältnis zwischen 1,5 und 2, besteht ein 30\%ig signifikant höheres Risiko von Komplikationen betroffen zu sein, als wenn das Verhältnis <1,5 beträgt $(\mathbf{P}=\mathbf{0 . 0 9 5})$. Ist das Verhältnis $>2$, bestand kein signifikanter Zusammenhang (siehe Tabelle 37, Zeile 1).

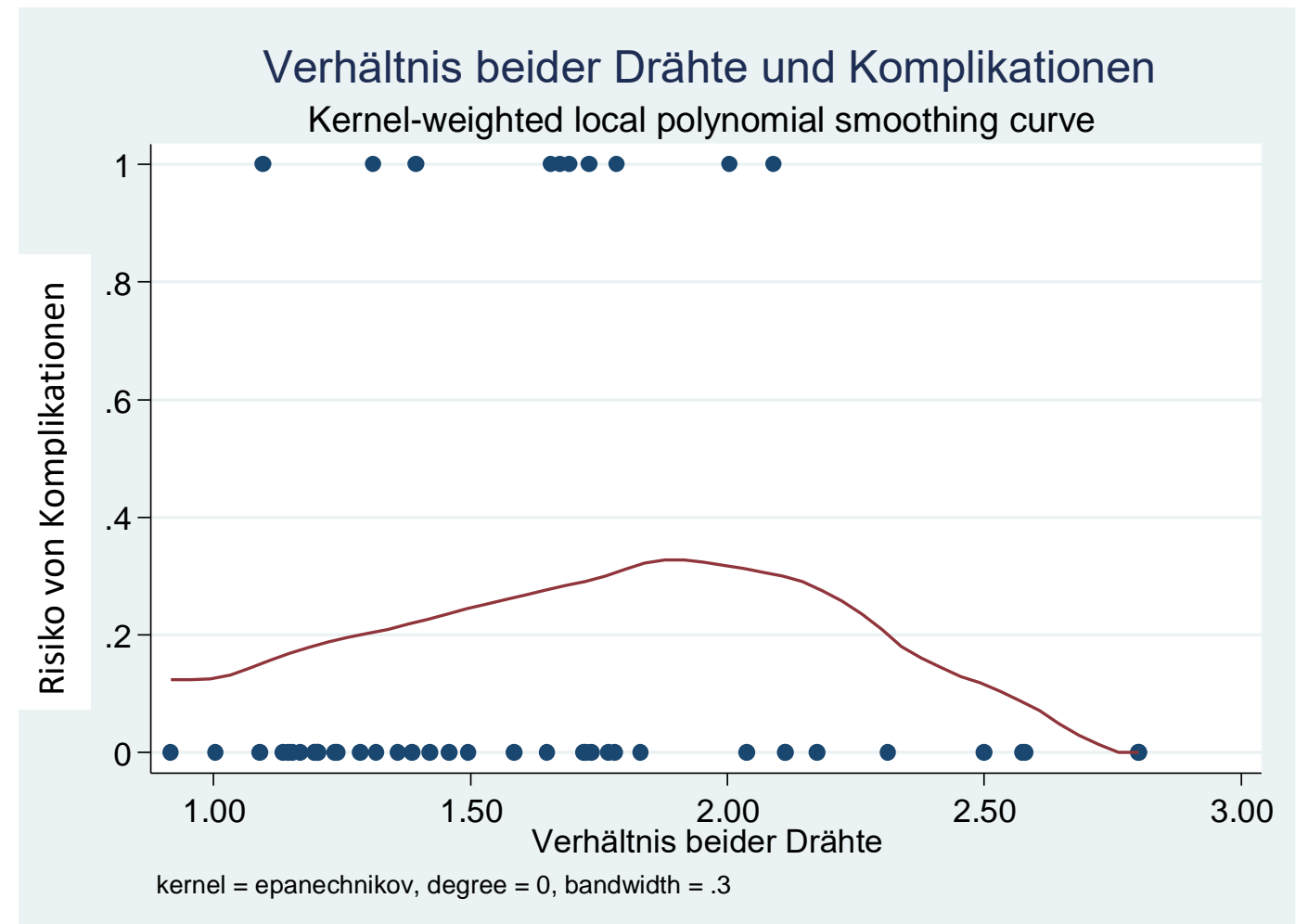

Abbildung 43: Lokale polynomiale Kernel-Regression. Der Zusammenhang zwischen Komplikationen und dem Verhältnis Markraumdurchmesser zu Drahtdicke wird dargestellt.

\subsubsection{Einflussfaktor: Unfallgeschehen}

Von den Patient(inn)en, bei denen die Unfallursache bekannt war, erlitten ein Fünftel (21/99) Komplikationen. Die meisten verunfallten bei einem Sturz aus einer Höhe von unter einem Meter (19\%). Die zweithäufigsten Unfallgeschehen mit Komplikationen waren mit jeweils $14 \%$ der Sturz aus einer Höhe von ein bis drei Metern oder ein Fahrradunfall. Siehe Tabelle 38 für die Häufigkeit von Komplikationen nach den jeweiligen Unfallmechanismen. 
Da sich besonders die unter drei Jahre alten Patient(inn)en eine Fraktur bei einem Sturz aus einer Höhe von unter einem Meter zugezogen hatten, wurden die Ergebnisse mit einem Multi-Variablen-Modell kontrolliert. Demnach hatten Patient(inn)en im Alter von drei bis achteinhalb Jahren ein 31\% geringeres Komplikationsrisiko als jüngere Patient(inn)en unter drei Jahren (Koeffizient -0.31, T-Wert -2.39, siehe Tabelle 39).

Aus Tabelle 39 lässt sich ebenfalls entnehmen, dass Frakturen, entstanden im Rahmen eines Anpralltraumas, mit einer 53\% höheren Wahrscheinlichkeit komplikationsreich verliefen als Frakturen durch einen Sturz aus einer Höhe von bis zu einem Meter (Koeffizient 0.53, T-Wert 1.29). Verunfallt als Fahrradfahrer(in), war das Komplikationsrisiko um 20\% höher (Koeffizient 0.19, T-Wert 0.63). Die beiden letzten Ergebnisse waren jedoch nicht signifikant.

\subsubsection{Einflussfaktor: Zeitraum zwischen Operation und Metallentfernung}

In 47 Fällen konnte neben dem Operationsdatum auch der Zeitpunkt der Metallentfernung erhoben werden. 72\% dieser Patient(inn)en (34/47) hatten sich das Implantat nach weniger als 55 Wochen entfernen lassen. In diesem Zeitraum traten auch die meisten Komplikationen auf, jedoch konnten keine signifikanten Zusammenhänge festgestellt werden. Verblieb ein Kraftträger für länger als 100 Wochen im Körper, traten keine Komplikationen auf. Die Beobachtungen sind Abbildung 44 zu entnehmen, die Ergebnisse der quadratischen Regression finden sich im Anhang (Tabelle 40).

Die Hypothese, dass Komplikationen zu einer zügigeren Metallentfernung führen, konnte demnach nicht bestätigt werden, da die Ergebnisse dem Signifikanztest nicht standhielten. 


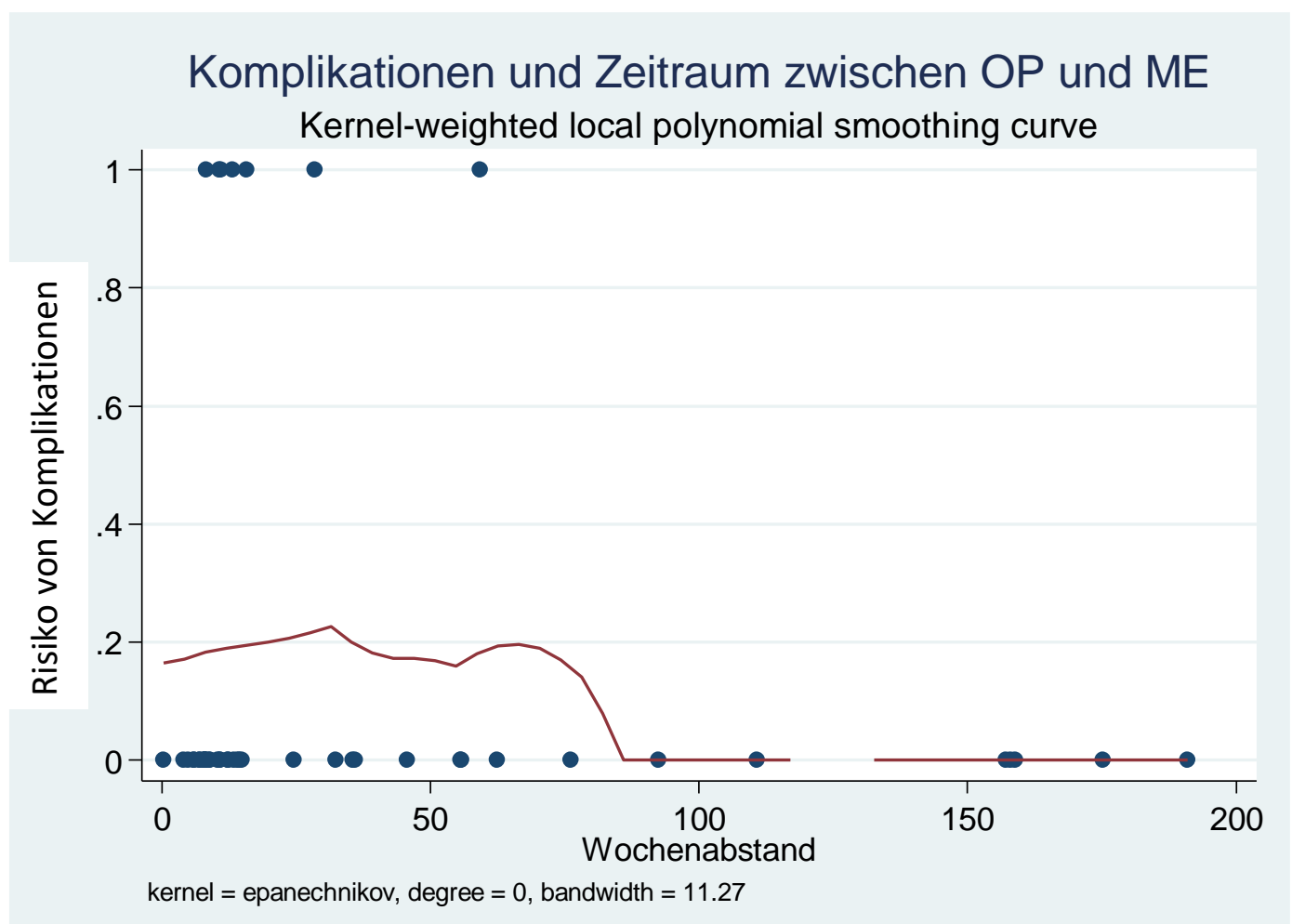

Abbildung 44: Kernel-Regression von postoperativen Komplikationen und des Zeitraumes zwischen Metallimplantation und -entfernung.

\subsubsection{Einflussfaktor: Liegedauer}

Statistisch scheint die Liegedauer das Komplikationsrisiko zu beeinflussen (siehe Tabelle 41). Es ist jedoch davon auszugehen, dass hier ein Kausalitätsproblem vorliegt und die Liegedauer durch die Komplikationen erhöht wird.

So beträgt die Liegedauer bei Patient(inn)en mit Komplikationen durchschnittlich 13 Tage, bei Patient(inn)en ohne Komplikationen zwölf Tage.

\subsubsection{Einflussfaktor: pathologische Frakturen, Begleiterkrankungen}

Dreizehn Erkrankte des Patient(inn)enkollektivs hatten eine pathologische Fraktur erlitten. Ihren Brüchen war ein nicht adäquates Trauma vorausgegangen. In diesen Fällen bestand ein um $10 \%$ signifikant höheres Komplikationsrisiko ( $P=0.01)$. Signifikanztest siehe Tabelle 42 im Anhang. 
Tabelle 12: Komplikationswahrscheinlichkeiten für Patient(inn)en mit pathologischen Frakturen (1) und Patient(inn)en mit Frakturen, denen ein adäquates Trauma vorausgegangen war (2). Pathologische Frakturen erhöhten das Komplikationsrisiko um 10\%. Patient(inn)en mit nicht pathologischen Frakturen erlitten in 20\% (18/88) der Fälle Komplikationen, Patient(inn)en mit pathologischen Brüchen hingegen zu 31\% (4/13).

\begin{tabular}{|c|c|c|c|}
\hline $\begin{array}{r}\text { pathologis } \\
\text { che \# } \\
\text { absolut } \\
0=\text { nein, } \\
1=j a\end{array}$ & $\begin{array}{r}\text { Komplikat } \\
0\end{array}$ & 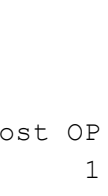 & Total \\
\hline 0 & $\begin{array}{r}70 \\
79.55\end{array}$ & $\begin{array}{r}18 \\
20.45\end{array}$ & $\begin{array}{r}88 \\
100.00\end{array}$ \\
\hline 1 & $\begin{array}{r}9 \\
69.23\end{array}$ & $\begin{array}{r}4 \\
30.77\end{array}$ & $\begin{array}{r}13 \\
100.00\end{array}$ \\
\hline Total & $\begin{array}{r}79 \\
78.22\end{array}$ & $\begin{array}{r}22 \\
21.78\end{array}$ & $\begin{array}{r}101 \\
100.00\end{array}$ \\
\hline
\end{tabular}

Fast ein Viertel der Patient(inn)en (24/101) litten an weiteren Erkrankungen neben der Femurschaftfraktur. Anders als erwartet, war für sie die Wahrscheinlichkeit, Komplikationen zu erleiden, niedriger (17\%) als für Patient(inn)en ohne Begleiterkrankungen (23\%). Siehe auch Tabelle 13.

Tabelle 13: Patient(inn)en mit und ohne Begleiterkrankungen und der jeweilige Anteil der Patient(inn)en, der Komplikationen erlitt (mit Begleiterkrankung 4/24, ohne Begleiterkrankungen 18/77 Patient(inn)en).

\begin{tabular}{r|rr|r} 
& Komplikation & post & OP \\
BegleitD & 0 & 1 & Total \\
\hline \multirow{2}{*}{0} & 59 & 18 & 77 \\
& 76.62 & 23.38 & 100.00 \\
\hline \multirow{2}{*}{1} & 20 & 4 & 24 \\
& 83.33 & 16.67 & 100.00 \\
\hline \multirow{2}{*}{ Total } & 79 & 22 & 101 \\
& 78.22 & 21.78 & 100.00
\end{tabular}




\section{Diskussion}

Ziel dieser Arbeit ist es, die Versorgungsrealität von kindlichen Oberschenkelschaftfrakturen an den Traumazentren der Universitätsmedizin Göttingen und Magdeburg (insgesamt 101 Kinder) abzubilden sowie die Risikofaktoren für Komplikationen zu analysieren. Als wichtige Ergebnisse der statistischen Auswertung sind zu nennen:

- Bei 78,22\% der Patient(inn)en verlief die Behandlung komplikationsfrei und mit guten Endergebnissen. 21,78\% der Erkrankten klagten über Komplikationen - den meisten von ihnen waren ESINs implantiert worden (in 68,12\% der Fälle). Zwei Kinder waren von Fehlstellungen betroffen, die nach von Laer (2007) als intolerabel gelten (Varusfehlstellung $15^{\circ}$ und Beinverkürzung um 2,5 cm nach Therapie mit ESINs; Rekurvation nach Plattenosteosynthese).

- Die besten Chancen für einen komplikationslosen Verlauf bestehen, unabhängig von der Therapie, für Patient(inn)en im Alter von vier bis acht Jahren (nicht signifikant).

- Die konservative Therapie weist ein signifikant $(\mathrm{P}=0.04)$ geringeres Komplikationsrisiko auf als die operative. Die mittlere Wahrscheinlichkeit, dass Patient(inn)en unter Komplikationen leiden, beträgt für konservativ behandelte Kinder 15\%; sie liegt höher mit $23 \%$ für operierte Kinder.

- Im Falle einer operativen Behandlung erweist sich das Alter als ein Risikofaktor, der bereits bis zu 20\% für die Variationen von Komplikationen verantwortlich ist. Das geringste Komplikationsrisiko besteht für die Drei- bis Achteinhalbjährigen: Ihr Risiko ist signifikant um 28\% ( $P=0.015)$ geringer als das der unter Dreijährigen. Die Zwölf- bis Dreizehnjährigen weisen wiederum ein um 26,5\% ( $P=0.066)$ signifikant höheres Risiko auf als die unter Dreijährigen.

- Werden den Patient(inn)en elastisch stabile intramedulläre Nägel eingesetzt, spielt neben dem Alter auch das Verhältnis von Markraumdurchmesser zu Drahtdicke eine signifikante Rolle. Liegt das Verhältnis zwischen 1,5 und 2, steigt das Komplikationsrisiko um 30\% $(P=0.095)$, verglichen mit einem Verhältnis von $<1,5$.

\subsection{Quantität und Qualität der Komplikationen im Patient(inn)en- kollektiv}

Ein Großteil der Patient(inn)en (78,22\%) erlebte einen komplikationsfreien Therapieverlauf mit guten Endergebnissen. Circa ein Fünftel der Erkrankten klagte über Komplikationen, den meisten von ihnen (68,12\%) waren ESINs implantiert worden. Bei zwei Kindern wurden intolerable Fehlstellungen festgestellt. Insgesamt 
beträgt die mittlere Wahrscheinlichkeit unter Komplikationen zu leiden, für konservativ behandelte Kinder $15 \%$ und ist somit signifikant $(P=0,04)$ geringer als für operierte Kinder (23\%). In der Literatur sind vergleichbare Studien mit ähnlichen, aber auch abweichenden Ergebnissen zu finden:

Poolman et al. (2006) analysierten 33 Studien mit insgesamt 2422 Patient(inn)en, die sich eine Femurschaftfraktur zugezogen hatten. Nach ihren Berechnungen sei ein operatives Verfahren komplikationsärmer im Vergleich zu einer konservativen Behandlung (Odds Ratio 0,74). Dies geht einher mit der Feststellung, dass die ESIN-Behandlung insgesamt ein niedrigeres Komplikationsrisiko berge als eine konservative Gips- oder Extensionsbehandlung (Odds Ratio 0,47).

Buechsenschuetz et al. (2002) verglichen den Heilungsverlauf von 68 Kindern mit Femurschaftfrakturen (drei- bis 14jährig). Bei ihnen ist die Komplikationsrate für die konservativ Behandelten mit Gips- und Extensionstherapie (26 Kinder) doppelt so hoch im Vergleich zu denen, die eine Federnagelung erhielten (44\% vs. 22\%, jedoch nicht signifikant).

Bei beiden Studien ist die konservative Therapie mit mehr Komplikationen verknüpft als die operative. Die vorliegende Arbeit kommt zu einem gegenteiligen Ergebnis.

Vergleicht man die Ergebnisse der vorliegenden Arbeit mit denen von Maier et al. (2003) auf der Ebene der konservativen und operativen Therapie, so scheint es auf den ersten Blick, als ob sich die Aussagen entsprechen 4 . Auf der Ebene der verschiedenen operativen Verfahren zeigen sich jedoch sehr deutliche Unterschiede, die im Folgenden erläutert werden. Die differenzierte Betrachtung ist notwendig, da nahezu $70 \%$ der postoperativen Komplikationen in dieser Arbeit auf ESINs entfallen. Innerhalb der operativen Verfahren kommen Maier et al. und die vorliegende Arbeit zu gegensätzlichen Ergebnissen. Dieses Phänomen trifft auch für die Studie von Sela et al. (2013) zu.

Von besonderem Interesse im Kontext dieser Arbeit sind die Studien von Jauquier et al. (2010) und Prata do Nascimento et al. (2013) zum einen wegen ihres Aufbaus und zum anderen auf Grund ihrer Ergebnisse: Die Komplikationsrate für beide Behandlungsansätze, operativ wie konservativ, liegt jeweils ähnlich hoch.

\footnotetext{
${ }^{4}$ Maier et al. (2003) untersuchten 101 Patient(inn)en und stellten folgende Komplikationsraten fest: $14 \%$ bei konservativer Therapie, $6 \%$ bei Behandlung mit ESIN, $44 \%$ bei Behandlung mit Fixateur externe. (Beinlängendiskrepanzen $>1 \mathrm{~cm}$ wurden separat aufgeführt und betrugen $4 \%$ bei konservativ, $8 \%$ bei den mit ESIN und $24 \%$ bei den mit Fixateur externe behandelten Kindern.) Insgesamt würde die konservative Therapie bessere Ergebnisse zeigen als die operative Therapie. Differenziert man jedoch bei den Behandlungsoptionen, so wäre die ESIN-Technik die komplikationsärmste Therapie.
} 
Jauquier et al. (2010) legten in ihrer Studie den Fokus auf Patient(inn)en mit Femurschaftfrakturen im Alter von null bis vier Jahren. 19 Kinder wurden mit Gips und 27 Kinder mit ESINs behandelt. Die Komplikationsrate (Gips-10,5\% vs. ESIN-14,8\%) sowie die Ausheilungsrate sind für beide Gruppen ähnlich hoch. Hinsichtlich unterschiedlicher Beinlängen um mehr als $2 \mathrm{~cm}$ zeigte die ESIN-Technik bessere Ergebnisse (Gips 26,3\% vs. ESIN 18,2\%).

Prata do Nascimento et al. (2013) verglichen die konservative mit der ESIN-Therapie bei zwei jeweils gleich großen Patient(inn)engruppen (je 30 Kinder zwischen fünf und 13 Jahren). Die Komplikationsrate betrug in beiden Gruppen $10 \%{ }^{5}$, jedoch unterschied sich die Häufigkeit der Beinlängendiskrepanzen erheblich: Bei 13,3\% der konservativ behandelten Kinder konnte eine Beinverlängerung (Overgrowth) festgestellt werden, hingegen waren es $60 \%$ bei den mit ESIN behandelten Kindern.

Die Autoren beider Studien kommen zu dem Schluss, dass die konservative Therapie sich nicht nachteilig, bei Jauquier et al. (2010) sogar vorteilhaft, auswirkt. Sie stützen somit die Ergebnisse der vorliegenden Arbeit. Beide Studien weisen eine insgesamt niedrigere Komplikationsrate aus, jedoch ist auch die wesentlich kleinere Proband(inn)enzahl zu beachten. Hinsichtlich der separat aufgeführten Beinlängendiskrepanzen kommen nur Prata do Nascimento et al. (2013) zu ähnlichen Ergebnissen wie diese Arbeit. In der Studie von Jauquier et al. (2010) hingegen erweist sich die Therapie mit Gips als nachteilig.

Von welchen Komplikationen waren die Patient(inn)en betroffen?

Wachststumsstörungen sind mit $42,11 \%$ die häufigste Komplikation in der vorliegenden Untersuchung. Bei der Hälfte der Betroffenen ist bekannt, dass es sich um ein überschießendes Wachstum handelt, also eine Beinverlängerung stattgefunden hat. Bei den übrigen war lediglich festzustellen, dass unterschiedliche Beinlängen vorlagen. Dieses Phänomen ist bekanntermaßen mit Hyperperfusion der Epiphysenfuge und intensiven Remodelling-Vorgängen $\mathrm{zu}$ erklären, die $\mathrm{zu}$ Längenwachstum führen. Auch langwieriges Reponieren oder Manipulieren der Frakturzone stimuliert das Wachstum. Nach von Laer (2007) ist bei Patient(inn)en unter zehn Jahren mit einer Beinverlängerung (Overgrowth) und bei den über Zehnjährigen mit einer Beinverkürzung zu rechnen. Ob bei den Patient(inn)en im untersuchten Kollektiv besonders lange oder wiederholt reponiert werden musste, ist nicht bekannt. Bei allen operativen Eingriffen am kindlichen Knochen sind sich die Chirurgen bewusst, dass besonders die Wachstumsplatte geschont werden muss. Bei

\footnotetext{
${ }^{5}$ alle Komplikationen außer Beinlängendiskrepanzen
} 
der elastisch stabilen intramedullären Nagelung wird als Eintrittsstelle ein Punkt circa $2 \mathrm{~cm}$ oberhalb der Epiphysenfuge ausgewählt. Wird diese Stelle zu weit distal bzw. proximal vom Schaft ermittelt, kann es zur Epiphysenfugenverletzung kommen. Bei der Betrachtung der Ergebnisse ist ebenfalls zu beachten, dass nicht bekannt ist, ob diese Kinder schon vor der Fraktur an Beinlängendifferenzen litten - nach Heimkes und Günther (2014) und Niethard (2010) sind 30\% der Kinder von einer idiopathischen Beinlängendifferenz betroffen. So kann es schon bei geringem Mehr- oder Minderwachstum zu Overgrowth bzw. einer Beinverkürzung von pathologischem Ausmaß kommen. Um diese Komplikation differenzierter bewerten zu können, wären die absoluten Maße postoperativ sowie nach dem Abschluss des Wachstums notwendig.

Die Häufigkeit von Wachstumsstörungen wird in der Literatur mit $0 \%$ bis $60 \%$ angegeben (Maier et al. 2003; Sela et al. 2013; Jubel et al. 2004; Moroz et al. 2006; Prata do Nascimento et al. 2013). Die Komplikationsrate im untersuchten Kollektiv beträgt $42,11 \%$ und liegt damit im oberen Drittel der vorgenannten Angaben.

Die zweithäufigste Komplikation, über die Patient(inn)en im untersuchten Kollektiv klagten, hat vermutlich ihren Ursprung in zu lang überstehenden Nagelenden bei der ESIN-Technik: 36,84\% litten unter „passagerer Einschränkung der Kniebeweglichkeit“, also unter einer Beugehemmung und/oder einem Streckdefizit. Dieses Problem entsteht laut Metaizeau (2004) durch die Irritation des Musculus vastus medialis oder laterialis und kann bis einige Monate nach der Metallentfernung anhalten. Dietz (1997) sowie Dietz und Schlickewei (2011) weisen darauf hin, dass diese Komplikation zu vermeiden sei durch eine ausreichend lange Hautinzision und Spaltung der Faszie. In der Literatur wird über diese Komplikation kaum berichtet. Lediglich in zwei Studien wird dieses Phänomen beschrieben: In einer Untersuchung von Moroz et al. (2006) litten $0,9 \%$ der Patient(inn)en unter eingeschränkter Kniebeweglichkeit ${ }^{6}$, bei Tomaszewski and Gap (2014) 1,63\%?. Im Kollektiv der vorliegenden Arbeit liegt der Wert bei $36,84 \%$ und damit mehr als 22 mal so hoch. Dieser Wert liegt vermutlich zu hoch, da keine genauen Angaben zu ermitteln waren über das Ausmaß der Bewegungseinschränkung im Knie. Es ist möglich, dass diese Komplikation angegeben wurde auch bei minimaler Einschränkung. Für eine qualifizierende Aussage wäre eine weiterführende Untersuchung notwendig.

\footnotetext{
${ }^{6}$ zwischen $10^{\circ}$ und $110^{\circ}$

${ }^{7}$ mit einer Beweglichkeit von $10^{\circ}$ bis $90^{\circ}$ bzw. $15^{\circ}$ bis $75^{\circ}$
} 
Während die postoperativen Komplikationen in den Studien differieren, werden in fast allen Studien Hautirritationen an Eintrittsstellen der ESINs genannt (Lascombes et al. 2006; Sela et al. 2013; Buechsenschuetz et al. 2002; Jauquier et al. 2010; Narayanan et al. 2004; Moroz et al. 2006; Prata do Nascimento et al. 2013; Jubel et al. 2004). Meist sind die Drahtenden ungenügend gekürzt und irritieren darüber liegendes Weichteilgewebe oder die Drähte migrieren aus dem Knochen und perforieren die Haut. Als Folge könnten sich Haut, Weichteile und der Knochen infizieren oder es könnte zu einer Stabilitätsminderung der Frakturzone kommen (Bandyopadhyay und Mekherjee 2013; Reynolds et al. 2012; von Laer et al. 2013).

Im untersuchten Kollektiv waren nur 1,98\% (2/101) der Erkrankten von Hautperforationen betroffen. Dies entspricht $10,53 \%$ aller Patient(inn)en mit postoperativen Komplikationen.

Jubel et al. (2004) empfehlen neben der ausreichend breiten Faszienspaltung $(2 \mathrm{~cm})$ die Verwendung eines speziellen Stößels aus dem TEN-Set der Firma Synthes (Bochum) um diese Komplikation zu vermeiden. Sie befürworten, die Drähte nach der korrekten Platzierung im Knochen noch einmal zurückzuziehen und um circa $3 \mathrm{~cm}$ zu kürzen und sodann die Drahtenden mit dem genannten Stößel ausreichend tief in den Weichteilen zu versenken.

Um diese Art von Komplikationen zu vermindern, entwickelte die Arbeitsgruppe für Osteosynthese (mit Sitz in der Schweiz) so genannte End Caps. Sie werden auf das circa $1 \mathrm{~cm}$ überstehende Drahtende aufgesetzt. Über $\mathrm{ihr}$ Gewinde wird anschließend der Nagel in der Kortikalis verankert. Die Anwendung wird in der Leitlinie der Deutschen Gesellschaft für Kinderchirurgie (publiziert von AWMF im Jahr 2014) ${ }^{8}$ empfohlen. Nectoux et al. (2008) veröffentlichten eine Untersuchung an zehn Kindern, die mit ESINs und End Caps versorgt wurden und exzellente Behandlungsergebnisse aufwiesen. Insbesondere traten keine Hautirritationen, Schmerzen oder Infektionen auf. Außerdem wurde die Metallentfernung erleichtert und dadurch große Narben vermieden. Die Autoren merken an, dass durch die größere Stabilität womöglich weniger Mikrobewegung stattfindet und es zu einer verzögerten Kallusbildung kommen könnte. Bei den untersuchten Patient(inn)en beobachteten sie eine weniger stark ausgeprägte Kallusbildung als bei vorherigen Nagelungen ohne End Caps. Es entstanden daraus jedoch keine Komplikationen für die Kinder. ${ }^{9}$ Kaiser et al. (2011) prüften die Stabilität durch Endkappen mit einem Spiralfraktur-Modell in vitro und

\footnotetext{
${ }^{8}$ http://www.awmf.org/leitlinien/detail/II/006-016.html, Zugriff am 15.08.2016

${ }^{9}$ Nur ein(e) Patient(in) benötigte einen Monat länger bis zur vollständig belastungsfähigen Frakturkonsolidierung (Nectoux et al. (2008)).
} 
konnten keine zusätzliche Stabilität im Vergleich zur Nagelung ohne Endkappen feststellen.

Malunion, sekundäre Dislokation und Malreduktion traten jeweils ein- oder zweimal (0,99\% oder 1,98\%) im Krankengut auf. Dies ist wenig im Vergleich zu anderen Studien: (Narayanan et al. 2004) litten unter einer Malunion und $8 \%$ in der Studie von Lascombes et al. (2006). Die Wahl einer falschen Implantatgröße (z.B. zu dünne ESINs) oder das falsche Repositionsverfahren können zu solchen Komplikationen führen.

Die Ursachen von hypertrophischen Narben/Keloiden (zweimal im Patient(inn)enkollektiv), Wundheilungsstörungen und Pin-Trakt-Infektionen (je einmal) sind nicht eindeutig auszumachen. Beides kann auf Grund einer genetischen Prädisposition oder anderer Grunderkrankungen hervorgerufen werden und/oder durch nicht ausreichend hygienische Bedingungen.

Ein zweieinhalbjähriger Junge mit offener Spiralfraktur erlitt ein Kompartmentsyndrom, nachdem er mit ESINs versorgt worden war. Diese Komplikation ist jedoch nicht typisch für die intramedulläre Nagelung, sondern muss im Kontext mit der offenen Fraktur gesehen werden. Der Bruch führte vermutlich zu starkem Einbluten ins Gewebe, wo sich der Druck auf pathologische Werte erhöhte. In nur einer weiteren Studie (Sink et al. 2005) wurde ein Kompartmentsyndrom nach einer ESIN-Behandlung verzeichnet.

Über folgende weitere Komplikationen der ESIN-Behandlung wird in der Literatur berichtet: tiefe Wundinfektionen, Hautnekrose, Implantatbruch, Synovitis, neurologische Ausfälle, Refraktur, Osteomyelitis, Lungenembolie (Tomaszewski and Gap 2014; Bandyopadhyay und Mekherjee 2013; Lascombes et al. 2006; Narayanan et al. 2004; Moroz et al. 2006; Buechsenschuetz et al. 2002). Keine dieser Komplikationen trat im Krankengut dieser Arbeit auf.

Im vorliegenden Patient(inn)enkollektiv sind sechs Revisionen zu verzeichnen (5,94\%). Insgesamt fünf Patient(inn)en waren betroffen. Bei einem Patienten bzw. einer Patientin wurde der Therapieansatz mit ESINs trotz Re-Operation beibehalten, in den übrigen Revisionsfällen wurde das Therapieverfahren gewechselt. Dabei war auffällig, dass bei zwei Patient(inn)en auch nach Revision weiterhin Komplikationen bestanden.

Die Re-Operationsrate bei einer operativen Therapie war mit 5,2\% (4/77) halb so hoch wie bei der konservativen Therapie mit 10,53\% (2/19). In diesen zwei Fällen wurde von der konservativen Therapie auf ESINs gewechselt. Die Re-Operationsraten verschiedener operativer Verfahren unterscheiden sich jedoch deutlich voneinander: 
Je zweimal wurden Fixateur externe (2/3 bzw. 66,67\%) oder ESINs (2/62 bzw. 3,23\%) ersetzt. Diese Beobachtung zeigt, dass mehr als die Hälfte der Kinder, die mit einem Fixateur externe behandelt worden waren, erneut operiert werden mussten.

Um diese Aspekte differenzierter zu betrachten, sind die dieser Studie zu Grunde liegenden Daten kaum geeignet (z.B. Stichprobengröße und Mehrfachnennungen). Vergleicht man die oben genannten Zahlen mit der Literatur, werden die eigenen Beobachtungen bestätigt und es ergibt sich folgendes Bild:

Ramseier et al. (2010) berichten über die Häufigkeiten von Re-Operationen bei 104 operativ versorgten Kindern mit Oberschenkelfrakturen. Auch in ihrem Patient(inn)enkollektiv war die Notwendigkeit einer Re-Operation bei den mit Fixateur externe behandelten Kindern am häufigsten - in 52\% der Fälle (16/33). Kinder, denen ESINs implantiert worden waren, benötigten in $8 \%$ der Fälle eine zweite operative Behandlung.

Ähnliche Ergebnisse sind 2004 von Jubel et al. veröffentlicht worden. Sie notieren drei Korrekturoperationen bei 47 Kindern, die mit ESINs behandelt worden waren. Der Anteil ist mit $6,38 \%$ gering, er ist jedoch doppelt so hoch verglichen mit dem den Patient(inn)en der vorliegenden Arbeit.

Bei Sink et al. (2005) fällt die Revisionsrate vergleichsweise hoch aus. Sie untersuchten die Re-Operationsrate von 39 Kindern mit Femurfrakturen, die mit elastisch stabilen Titannägeln (ESINs) behandelt wordet waren: 21\% mussten sich ein zweites Mal einer operativen Therapie unterziehen. (Das ist eine der höchsten Raten, die in der Literatur für die ESIN-Therapie beschrieben werden.)

Einzelne Veröffentlichungen beschreiben revisionspflichtige Komplikationen sowohl beim konservativen als auch beim operativen Therapieansatz: Sela et al. (2013) berichten über die Revisionsnotwendigkeit lediglich bei Behandlung mit Gips oder Fixateur externe. In zehn Fällen musste erneut repositioniert und ein Gips angelegt werden (10/151 bzw. 6,62\%). In einem weiteren Fall wurde auf ein operatives Verfahren gewechselt (Konversionsrate $=0,66 \%$ ). Die Revisionsrate der konservativ behandelten Kinder $(6,62 \%)$ fällt niedriger aus als im hier untersuchten Kollektiv $(10,53 \%)$.

Ein noch deutlicherer Unterschied besteht bei den mit Fixateur externe Behandelten. Lediglich bei einem von 14 Patient(inn)en, die Sela et al. (2013) untersuchten, war eine Reoperation notwendig $(7,14 \%$ bei $n=14)$ - dies ist deutlich weniger als im Patient(inn)enkollektiv dieser Arbeit $(66,67 \%$ bei $n=3)$. 


\subsection{Alter als Risikofaktor für Komplikationen}

Das untersuchte Patient(inn)enkollektiv umfasst eine Altersspanne von drei Monaten bis 15 Jahren und sieben Monaten (Durchschnittsalter 7,05 Jahre). Um möglichst differenzierte Erkenntnisse bezüglich der Versorgung und der Komplikationen im Wachstumsalter zu gewinnen, wurden Patient(inn)en im Alter von null bis 16 Jahren berücksichtigt. Die Jüngsten (null bis drei Jahre) wurden aus folgenden Gründen einbezogen: Obwohl in der Leitlinie der Deutschen Gesellschaft für Kinderchirurgie ${ }^{10}$ die operative Therapie erst ab dem dritten Lebensjahr empfohlen wird, stellen Strohm und Schmittenbecher (2014) fest, dass bereits 51\% der unter Dreijährigen bei einer Femurschaftfraktur operativ behandelt werden ( $50 \%$ mit ESIN, $1 \%$ mit Fixateur externe).

Die Altershöchstgrenze wurde bei 16 Jahren festgelegt, da sich die Wachstumsfugen des proximalen und distalen Femurpols individuell zwischen dem 15. und 18. Lebensjahr schließen. (Mit dem Fugenschluss wäre eine Spontankorrektur ausgeschlossen.) Mit der gewählten Altershöchstgrenze sollte vermieden werden, dass Daten von Patient(inn)en mit einem ausgereiften Skelett in die Analyse eingehen.

Die gewählte Altersspanne dieser Studie konnte nur in einer weiteren Veröffentlichung gefunden werden (Sela et al. 2013).

Im Folgenden wird auf das Alter als Risikofaktor für Komplikationen detailliert eingegangen: $21,78 \%$ der Kinder litten unter Komplikationen. Die meisten von ihnen wurden operativ mit der elastisch stabilen intramedullären Nagelung (68,12\%) behandelt. Die besten Chancen für einen komplikationslosen Verlauf bestehen therapieunabhängig für die Vier- bis Achtjährigen (statistisch jedoch nicht signifikant).

Die Leitlinie ${ }^{11}$ mit ihrer Empfehlung, bis zum dritten Lebensjahr konservativ zu behandeln, wird durch diese Arbeit gestützt, auch wenn die unter Vierjährigen nicht zur komplikationsärmsten Gruppe zählen. Die konservative Behandlungsmethode stellte sich als signifikant komplikationsärmer im Vergleich zur operativen (15\% vs. 23\%) heraus. Differenziertere Angaben dazu können nicht gemacht werden, da bei 16 der 19 konservativ behandelten Kinder das Alter nicht zu ermitteln war. Es ist jedoch davon auszugehen, dass Kinder unter drei Jahren eher operiert, als über Dreijährige konservativ behandelt worden sind (vgl. Strohm und Schmittenbecher 2015) sowie Ergebnisse Kapitel 3.2 und 3.3: Anzahl der konservativ Behandelten=19, Anzahl der

\footnotetext{
${ }^{10}$ (S1-AWMF-Leitlinie, Stand 09/2014)

${ }^{11}$ Leitlinie der Deutschen Gesellschaft für Kinderchirurgie, siehe Fußnote 9
} 
unter Vierjährigen=36). Diese Schlussfolgerung bildet eine Tendenz ab und benötigt zu ihrer Validierung weitere Untersuchungen.

Im Unterschied dazu liegen für die operative Therapie signifikante Ergebnisse vor. Hier macht allein das Alter bis zu 20\% der Variationen der Komplikationen aus. Das höchste postoperative Komplikationsrisiko im Kollektiv besteht für die Zwölf-/DreizehnJährigen. Sie haben ein um 26,5\% höheres Risiko als die unter Dreijährigen. Das geringste Risiko besteht für die Drei- bis Achteinhalbjährigen, denn sie haben ein $28 \%$ niedrigeres Risiko als die unter Dreijährigen. Alle Ergebnisse sind signifikant.

Die unter Dreijährigen gehören also nicht zur risikoärmsten Gruppe, wenn sie operiert werden. Somit liegt die Schlussfolgerung auch hier nahe, dass die Jüngsten (null bis drei Jahre) konservativ therapiert werden sollten (siehe oben und Leitlinie). Biomechanisch und physiologisch gibt es eine Erklärung für häufige Frakturen im Kleinkindalter: Das Skelett ist noch nicht hinreichend verknöchert und robust, sodass die kindlichen Knochen bei einem Sturz leicht brechen können (Rewers et al. 2005). Eventuell ist dies auch der Grund für häufigere Komplikationen bei der Frakturheilung in diesem Alter.

Es kann weder ein einzelner Faktor (wie z.B. Unfallursache, Body-Maß-Indices, Fraktur-, Repositionsformen, Implantationsmaterial) noch eine Kombination von Faktoren ausgemacht werden, die das Komplikationsrisiko in diesen spezifischen Altersgruppen erhöhen - außer das Verhältnis Markraumdurchmesser zur Drahtdicke im Falle einer ESIN-Implantation. Es ist bemerkenswert, dass das Alter, in dem das höchste Komplikationsrisiko besteht, mit dem Alter hoher Wachstumsgeschwindigkeit zusammenfällt (dritte Wachstumsphase). Laut Niethard et al. (2009) liegt das Maximum des Wachstumsschubs bei Mädchen um das zwölfte und bei Jungen um das 14. Jahr des Skelettalters. Möglicherweise können die physiologischen Eigenschaften der pubertären Knochen das hohe Komplikationsrisiko der Zwölf-/Dreizehn-Jährigen erklären.

In nur einer weiteren Studie wurde das Alter als Risikofaktor für Komplikationen nachgewiesen: Moroz et al. (2006) untersuchten 234 kindliche Femurfrakturen, die mit ESINs behandelt wurden. Das Alter war ein signifikant unabhängiger Prädiktor für die Qualität des Endergebnisses und stützt die Resultate der vorliegenden Arbeit. Bei Moroz et al. (2006) beträgt die Odds Ratio für ein schlechtes Endergebnis 3,86, wenn die Wahrscheinlichkeit für ein schlechtes Endergebnis für die über Elfjährigen zu der der Jüngeren ins Verhältnis gesetzt wird. Somit unterliegen die unter Elfjährigen einem geringeren Risiko für ein schlechtes Therapieergebnis. Die Autoren erklären dieses Ergebnis teilweise mit den strengeren Kriterien, die bei Wachstumsstörungen über 
Zehnjähriger angelegt werden. So werden gleichartige Fehlstellungen im Alter unter zehn Jahren nicht als Komplikation gewertet, die bei den über Zehnjährigen als intolerable Achsfehlstellung oder Beinlängendifferenz gelten. Diese Erklärung greift allerdings nicht für das im eigenen Patientengut aufgetretene erhöhte Komplikationsrisiko (im Alter von zwölf/dreizehn).

Die Inzidenz von Femurschaftfrakturen ist generell niedrig und es ist sind keine Studien bekannt, die den Zusammenhang zwischen dem zwölften/dreizehnten Lebensjahr und dem Risiko für Komplikationen bei der operativen Behandlung von Femurschaftfrakturen erklären. Um Hypothesen abzuleiten, muss auf Untersuchungen mit anderen Schwerpunkten verwiesen werden.

Seit den 1990er Jahren wird in Studien wiederholt festgestellt, dass das Frakturisiko im Alter von zwölf bis 13 Jahren extrem ansteigt (besonders für eine distale Radiusfraktur) und dies nachweislich in Zusammenhang steht mit den Umbauvorgängen des Knochens während des finalen Wachstumsschubs (Parfitt 1994; McKay et al. 1998; Bonjour et al. 1994; Rauch 2012; Fournier et al. 1997; Chevalley et al. 2011; Chevalley et al. 2012; Kalkwarf et al. 2011; Bonjour und Chevalley 2014).

Fournier et al. (1997) sowie Bonjour und Chevalley (2014) beschreiben eine zeitliche Ungleichheit bei der Zunahme der Knochenlänge und Knochenmasse. Die größte Diskrepanz der beiden Maße tritt mit dem pubertären Wachstumsschub auf - bei Mädchen im Alter von elf bis zwölf, bei Jungen zwischen 13 und 14 Jahren (entspricht den Entwicklungsstufen P2-P3). Das Längenwachstum nimmt hier so schnell zu wie nie zuvor im Wachstumsalter. Um ein Jahr versetzt wird der Knochenmineralgehalt/die Knochendichte rasant angereichert, ebenfalls einzigartig schnell, und "holt" das relative Knochenmassendefizit "auf" (peak bone mineral content velocity, Bonjour und Chevalley 2014). Es wird daraus geschlossen, dass während des relativen Defizits des Knochenmineralgehalts, eine erhöhte Fragilität für kindliche/jugendliche Knochen besteht. Es ist nicht auszuschließen, dass die gleichen Faktoren zu einem erhöhten Komplikationsrisiko im Patientengut beitragen. Unter Umständen bieten ESINImplantate nicht ausreichend Stabilität in genau dieser pubertären Umbau- und Remodelling-Phase (Vergleiche auch 4.3).

Parfitt (1994) konstatiert, dass infolge des Wachstums bzw. für das Wachstum im metaphysären Bereich, kortikales Kalzium verwendet würde und somit die Kortikalis vorübergehend vermehrt porös sei. Es ist vorstellbar, dass eine poröse Kortikalis dem Anpressdruck durch ESINs nicht adäquat standhält und Komplikationen auftreten.

Denkbar ist auch, dass gerade dort, wo das Wachstum stattfindet (Metaphyse), die Drahtenden der Federnägel einen minimalen Wachstumsreiz setzen. In Kombination 
mit fraktur- und altersbedingten intraosseären Umbauten könnte dies zu unterschiedlichen Beinlängen führen. Dies könnte eine Erklärung sein für die häufigste Komplikation im Patient(inn)enkollektiv.

Die zweithäufigste Komplikation im untersuchten Patient(inn)enkollektiv sind Bewegungseinschränkungen im Knie. Dies kann zum einen durch ungenügend gekürzte Federnägel nach der Implantation verursacht sein ${ }^{12}$. Jedoch gibt es keine Berichte über besondere Schwierigkeiten dafür bei zwölf-/dreizehnjährigen Kindern. Eine andere Möglichkeit der Erklärung ist die von Niethard et al. (2009) erwähnte „Pubertätssteife“. Bei außergewöhnlich schnellem Längenwachstum der Röhrenknochen wächst der Skelettmantel nicht rasch genug mit. Dann sind Sehnen und Muskeln relativ verkürzt und Bewegungen eingeschränkt. Es ist zu vermuten, dass eine Kombination der genannten Faktoren (überstehende Nagelenden, irritierter Muskel und relativ verkürzte Sehnen) zu dem hohen Komplikationsrisiko der Zwölf-/Dreizehnjährigen führt.

In der Literatur werden Kalzium, Vitamin D, die Menge von Proteinen in der Nahrung, das Alter bei Einsetzen der Pubertät, Thanner Stadien und Bewegung als Einflussfaktoren auf die Knochenstabilität im Jugend- und Erwachsenalter diskutiert (Wang et al. 2009; Xu et al. 2009; Chevalley et al. 2012; Ferrari et al. 2006; Rauch 2012; Bonjour und Chevalley 2014; McKay et al. 1998; Parfitt 1994). Vom untersuchten Patientengut liegen keine Informationen bezüglich der Ernährung, Knochendichte, Pubertätsstadien oder sportlicher Aktivitäten vor. Mögliche Zusammenhänge sind deshalb nicht zu überprüfen. Es bedarf weiterer Untersuchungen, um festzustellen, ob diese Faktoren die Komplikationsrate beeinflussen.

Zusammenfassend lässt sich feststellen, dass die in den Leitlinien angeratene konservative Therapie für die unter Dreijährigen ihre Berechtigung hat. Bei wenigen der konservativ Behandelten konnte das Alter festgestellt werden - daher sind hier keine differenzierteren Aussagen möglich.

Die vorliegende Arbeit bestätigt, dass die operative Therapie mit ESINs ab dem dritten Lebensjahr grundsätzlich zu empfehlen ist - hier besteht das geringste Komplikationsrisiko. Dies gilt uneingeschränkt für die Kinder im Alter von drei bis achteinhalb Jahren. Für die Zwölf-/Dreizehnjährigen besteht das höchste Komplikationsrisiko bei einer operativen Therapie. Die altersspezifischen Knochenveränderungen und Besonderheiten während des Wachstums müssen bei der Therapie stärker beachtet werden.

\footnotetext{
12 Die Benutzung von End Caps könnte dieser Komplikation vorbeugen, siehe auch 4.1.
} 


\subsection{Verhältnis von Markraumdurchmesser zu Drahtdicke als Risikofaktor für Komplikationen bei der ESIN-Therapie}

Werden Patient(inn)en mit ESINs behandelt, spielt neben dem Alter auch das Verhältnis von Markraumdurchmesser zu Drahtdicke eine signifikante Rolle für das Komplikationsrisiko. Der Federnagel-Hersteller empfiehlt eine Drahtdicke zu wählen die jeweils mindestens 40\% des Markraumdurchmessers entspricht: Es werden in der Regle zwei ESINs eingesetzte, sodass dann 80\% der Markraumbreite ausgefüllt wären.

Die vorliegende Arbeit stützt diese Empfehlung. Bei einem Verhältnis von Markraumdurchmesser zu Drahtdicke zwischen 1,5 und 2, nimmt das Komplikationsrisiko verglichen mit dem Verhältnis $<1,5$ - um 30\% (P=0.095) zu.

Ist das Verhältnis zwischen 1 und 1,5 gewählt worden, also im komplikationsärmeren Bereich, füllen die Nägel die Markraumbreite zu $100 \%$ bis $75 \%$ aus. Je größer das Verhältnis von Markraumdurchmesser zur Drahtdicke, desto mehr unausgefüllter Markraum bleibt zurück. Für ausreichend Stabilität und Komplikationslosigkeit bei der Frakturheilung sollte der Markraum ausgefüllt sein. Wählen Operateure zu dünne ESINs, erhöht sich signifikant das Komplikationsrisiko für die Erkrankten.

Die Ergebnisse einer Studie von Flinck et al. (2015) bilden eine ähnliche Tendenz ab. Sie testeten am Modell die Stabilität von zwei und vier ESINs mit End Caps sowie vom Adoleszentennagel bei einer längsinstabilen Femurschaftfraktur (Markraumbreite $10 \mathrm{~mm}$ ). Am stabilsten erwies sich der Marknagel und fast ebenso stabil vier ESINs (à 3,00 mm). War der Markraum durch Implantate stärker ausgefüllt, so erwies sich das Frakturmodell als stabiler. Dies ist eine wichtige Beobachtung, besonders für die Behandlung von Patient(inn)en, die durch höheres Gewicht ihre (Femur-)Knochen belasten.

Aufschlussreich ist auch die Betrachtung der beiden Risikofaktoren Alter (siehe oben) und Verhältnis (Verhältnis von Markraumbreite zu ESINs) in Kombination. Während mit dem Alter bereits $20 \%$ der Komplikationen zu erklären sind, steigt der Anteil auf $30 \%$, sobald man das komplikationsreichere Verhältnis 1,5 bis 2 hinzunimmt. Ein Drittel der Komplikationen sind also durch diese beiden Risikofaktoren zu erklären. Da alle Ergebnisse signifikant sind, sollte eine Senkung des Komplikationsrisikos an diesen beiden Faktoren ansetzen.

Weitere Studien bestärken die Vermutung, dass eine Behandlung respektive ein Implantat mit höherer Stabilität als die ESIN-Therapie sich günstig auf die Komplikationsrate bei den Zwölf-/Dreizehnjährigen auswirkt.

Cooper et al. (2008) stellen fest, dass sich die Knochendichte eines jugendlichen Tibischaftes segmental intraindividuell unterscheidet: So weisen die lateral-posterioren 
und medial-posterioren Segmente die höchste Knochendichte auf, die anterioren die niedrigste. Die Autoren schließen daraus, dass Remodelling-Vorgänge im Knochen nicht gleichmäßig ablaufen, sondern die Mikroarchitektur an Belastung bzw. Gewicht (loading) angepasst wird. Informationen solcher Art liegen über die Kortikalis des Femurschaftes nicht vor, könnten jedoch hilfreich sein für die Erklärung der Komplikationsrate bei den operativen Therapien. Zu Beginn der Pubertät weist der kindliche Knochen durch vermehrten Umbau eine höhere Porosität auf (vergleiche 4.2). Es ist nicht auszuschließen, dass bei der Implantation mit zwei ESINs (wie bisher üblich) besondere Belastungsmuster innerhalb des Knochens auftreten und somit auch Remodellingprozesse in einer Weise beeinflusst werden, die in Komplikationen münden. Um diese Überlegungen zu überprüfen, bedarf es weiterer Untersuchungen. Sollte sich diese Annahme bestätigen, wäre abermals für Kinder in diesem Alter eine Therapie zu fordern, die für größerer Stabilität im Knochen sorgt.

Ein alternatives Implantat ist der Adoleszentennagel. Die Leitlinie weist speziell für Kinder, die mehr als $49 \mathrm{~kg}$ wiegen oder hoch gewachsen sind, auf diesen Marknagel hin. Er kann lateral am Trochanter major eingeführt werden um die schwere Komplikation einer avaskulären Kopfnekrose zu vermeiden. Studien zeigen sehr gute Behandlungsergebnisse bei dieser Methode - teilweise sogar bessere als bei der ESINTechnik (Reynolds et al. 2012; Keeler et al. 2009; Martus 2016)

Reynolds et al. (2012) verglichen die Komplikationsrate und Behandlungsergebnisse von Kindern, die mit ESINs oder einem Adoleszentennagel (ALFN) behandelt wurden. Die Rate der Komplikationen unterschied sich nicht signifikant und in der ALFN Gruppe traten keine schweren Komplikationen (major complications) auf. Die mit ALFN behandelten Kinder belasteten ihr operiertes Bein doppelt so schnell wie die mit ESIN behandelten. Kinder in der ALFN Kohorte waren älter und durchschnittlich schwerer. Dies schränkt die Bewertung der Ergebnisse jedoch nicht ein, da der Adoleszentennagel speziell entwickelt wurde für schwere und hoch gewachsene Patient(inn)en.

Martus (2016) erstellte eine Synopsis der aussagekräftigsten Patient(inn)enstudien mit ALFN: Diese Therapie zeigte gute Ergebnisse. Osteonekrose und Wachstumsstörungen würden nicht auftreten. So scheint der Adoleszentennagel eine gleichwertige Alternative zur ESIN-Technik zu sein. Als Mindestalter hält Martus (2016) das zehnte Lebensjahr als Richtwert fest, auch wenn die Jüngsten in den von ihm herangezogenen Studien erst sieben Jahre alt sind. ${ }^{13}$

\footnotetext{
${ }^{13}$ Keeler et al. (2009) sind der Auffassung, die Behandlung mit dem Adoleszentennagel sei eine sichere Therapie für Kinder ab neun Jahren.
} 
Es lässt sich festhalten, dass das Komplikationsrisiko bei einer ESIN-Implantation ansteigt, wenn der unausgefüllte Markraum mehr als 25\% beträgt. In Zusammenschau mit dem Alter als zusätzlichem Risikofaktor sollte eine alternative Therapie zur ESINTechnik für die Zwölf-/Dreizehnjährigen in Betracht gezogen und geprüft werden. Eine Alternative, die in bisherigen Studien gute Ergebnisse gezeigt hat, ist der Adoleszentennagel. Er wurde ursprünglich für übergewichtige Kinder und hoch gewachsene Jugendliche entwickelt. Möglicherweise bietet er auch normalgewichtigen, jüngeren Patient(inn)en eine komplikationsärmere Therapieoption.

\subsection{Grenzen der vorliegenden Dissertation}

Die Aussagen dieser Arbeit beziehen sich auf die Stichprobe $n=101$ und beanspruchen keine Allgemeingültigkeit. Die Stichprobe ist im Vergleich zu anderen Studien relativ klein, sie umfasst dennoch alle in den beiden Universitätsklinika Göttingen und Magdeburg dokumentierten Fälle (Klinikum Göttingen: 2006 bis 2013, Klinikum Magdeburg 2003 bis 2010, vgl. 2.1).

Die der Arbeit zugrunde liegenden Daten wurden an Hand der Krankenakten der Universitätsklinika Göttingen und Magdeburg erhoben und retrospektiv analysiert. Die Akten waren teilweise unvollständig und somit die Analysemöglichkeiten eingeschränkt. Zudem lagen eine Reihe von Bedingungsfaktore nicht vor, die eine weitergehende Analyse ermöglichet hätten. Wünschenswert wären zum Beispiel folgende Angaben gewesen: Vor dem Trauma bestehenden Beinlängendifferenz, Ernährung (Proteinmenge, Vitamin D, Kalzium), Knochendichte, Häufigkeit von sportlichen Aktivitäten oder jeweilige Entwicklungsstadien.

Es war zudem nicht nachzuvollziehen, ob Patient(inn)en Komplikationen erlitten und möglicherweise zur Behandlung ein anderes als das Ursprungskrankenhaus (Universitätsklinika Göttingen und Magdeburg) aufgesucht haben. Dadurch sind möglicherweise weniger Komplikationsfälle erfasst, als tatsächlich vorlagen. 


\section{$5 \quad$ Zusammenfassung}

Für die vorliegende Arbeit wurden retrospektiv Daten eines Patient(inn)enkollektivs ausgewertet, das 101 Kinder mit Femurschaftfrakturen umfasst. Die Erkrankten waren in der Universitätsmedizin Göttingen (1.1.2006 bis 1.11.2013) oder Magdeburg (1.5.2003 bis 31.12.2010) behandelt worden. Ziel dieser Studie war es, die Versorgungsrealität abzubilden, die Quantität und Qualität der Komplikationen zu erfassen und Risikofaktoren für die Komplikationen auszumachen.

Das Durchschnittsalter im Patientengut beträgt 7,05 Jahre (Altersspanne drei Monate bis 15 Jahre und drei Monate). 67,33\% der Erkrankten waren männlich. Die Unfallmechanismen unterschieden sich je nach Alter: Unter Dreijährige verunfallten zumeist beim „Toben/Spielen“ (25\%) oder durch einen Sturz $\leq 1$ m (17\%), Drei- bis Achteinhalbjährige zogen sich ein Quetschtrauma (17\%) zu oder stürzten beim Fahrradfahren (17\%) und über Achteinhalbjährige verletzten sich am häufigsten im Rahmen eines Verkehrsunfalls (15\% als Fußgänger, 12\% als Pkw- oder Businsass(inn)en).

Knapp ein Drittel (31,68\%) der Erkrankten zog sich eine Spiralfraktur zu, in jeweils $14,85 \%$ der Fälle traten Quer- und Schrägfrakturen auf sowie zu 6,93\% Mehrfragmentfrakturen. Bei 13/97 Kindern lag eine pathologische Fraktur vor und knapp ein Viertel der Patient(inn)en (23,76\%) wies Begleiterkrankungen auf. Die Liegedauer betrug im Durchschnitt 12,32 Tage.

Am häufigsten (77/101) wurde operativ behandelt, zumeist mit ESINs $(75,61 \%)$, Plattenosteosynthesen (13,42\%), Kirschner-Drähten oder Fixateur externe (jeweils 3,66\%) sowie jeweils einmal mit einem Marknagel und einer Schraube. Metallentfernung fand nach durchschnittlich 44 Wochen statt. Bei der elastisch stabilen intramedullären Nagelung wurde das Verhältnis von Markraumdurchmesser zur ESIN-Drahtdicke ermittelt: Durchschnittlich betrug das Verhältnis 1,77 (kleinster Quotient 1, größter 4,52).

Mehr als ein Fünftel aller Patient(inn)en klagten über Komplikationen: 42,11\% von ihnen über Wachstumsstörungen und 36,84\% über Einschränkungen der Kniegelenksbeweglichkeit. Weitere Probleme waren: Hautperforation, Kompartmentsyndrom, Malunion, sekundäre Dislokation, Pin Tract Infection, Wundheilunsgstörung, Malreduktion und hypertrophe Narbe (jeweils ein- oder zweimal). Zwei Kinder litten unter einer intolerablen Achsfehlstellung. Infolge von Komplikationen kam es fünfmal 
zum Verfahrenswechsel. Hervorzuheben ist, dass bei $\mathbf{7 8 , 2 2 \%}$ der Erkrankten die Behandlung ohne Komplikationen und mit gutem Endergebnis verlief.

Im Folgenden wurde der Einfluss verschiedener Parameter auf die Komplikationsrate überprüft. Kinder zwischen vier bis acht Jahren hatten therapieunabhängig die besten Chancen für einen komplikationsfreien Verlauf (nicht signifikant).

Da die konservative Therapie mit einem signifikant geringeren Risiko für Komplikationen einherging als die operative (15\% vs. $23 \%)$, ist bei den unter Dreijährigen leitliniengerecht weiterhin die konservative Therapie zu empfehlen. Differenziertere Angaben können nicht gemacht werden, da nur von einigen konservativ behandelten Kindern das Alter zu ermitteln war oder die Analyse keine signifikanten Ergebnisse erbrachte.

Bei der operativen Therapie war ein Risikofaktor das Alter. Damit konnten bereits $20 \%$ der Komplikationen erklärt werden. Das geringste Komplikationsrisiko bestand für die Drei- bis Achteinhalbjährigen, das höchste für die Zwölf-/Dreizehnjährigen. Den meisten der operativ Versorgten waren ESINs eingesetzt worden (68,12\%). Bei dieser Methode konnte ein weiterer Risikofaktor ermittelt werden: Das Verhältnis von Markraumdurchmesser zur Nageldicke. Betrug das Verhältnis zwischen 1,5 bis 2 und lagen somit neben den Kraftträgern im Knochen noch $25 \%$ bis $50 \%$ unausgefüllter Markraum vor, war das Komplikationsrisiko signifikant erhöht (verglichen mit dem Verhältnis $<1,5)$.

Die Kombination beider Risikofaktoren erklärt 30\% der Komplikationsrate. Bei der Bewertung müssen die biomechanischen Eigenschaften des pubertären Knochens beachtet werden: In der Literatur wird über eine stärkere Fragilität durch vermehrten Umbau während des Wachstumsschubs berichtet. Möglicherweise tragen die gleichen Eigenschaften zum Komplikationsrisiko der Zwölf-/Dreizehnjähren bei. Wachstumsstörungen treten häufig nach Frakturen oder wiederholten Repositionsversuchen durch eine gesteigerte Perfusion auf. Es ist nicht auszuschließen, dass durch die alterstypischen Remodellingvorgänge und durch zu dünne ESIN-Drähte Mikrobewegungen in erhöhtem Maße stattfinden, die ein Overgrowth stimulieren.

Auch die zweithäufigste Komplikation könnte durch eine pubertätstypische Besonderheit ausgelöst sein: Eingeschränkte Bewegungsfreiheit im Knie kann durch unzureichend gekürzte Nagelenden und/oder „Pubertätssteife“ (relativ verkürzte Sehnen und Muskeln im Vergleich zum rasant wachsenden Knochen) entstehen.

Um das Komplikationsrisiko zu senken, sollte auch an eine alternative Therapie, wie zum Beispiel den Adoleszentennagel, gedacht werden. Er bietet eine größere Stabilität 
und füllt eine größere Durchschnittsfläche aus als ESINs. Studien hierzu zeigen gute Endergebnisse, insbesondere weil Wachsstumstörungen oder avaskuläre Femurkopfnekrosen nicht auftraten. Obwohl der Adoleszentennagel für adipöse und hoch gewachsene Kinder entwickelt wurde, muss überprüft werden, ob er sich auch für normalgewichtige (prä-)pubertäre Kinder als komplikationsärmere Behandlungsmethode herausstellt.

Für die Drei- bis Achteinhalbjährigen, die Gruppe mit dem geringsten Komplikationsrisiko, scheint die ESIN-Behandlung die zu empfehlende Therapie zu sein. Diese Arbeit stützt somit in Teilen die Leitlinien der Gesellschaft für Kinderchirurgie. 


\section{$6 \quad$ Anhang}

\subsection{Fehlstellung nach Gresing}

Auch Gresing (2011) definiert Fehlstellungsgrenzen und differenziert stärker beim Alter der Kinder zum Unfallzeitpunkt. Die Grad- und Längenangaben für akzeptable Fehlstellungen sind Tabelle 14 zu entnehmen.

Tabelle 14: Akzeptable Fehlstellungen am Femurschaft nach Gresing (2011)

\begin{tabular}{l|l|l|l|l} 
Art der Fehlstellung & \multicolumn{5}{l}{ akzeptable Fehlstellungen je nach Altersgruppe } \\
& $\begin{array}{l}\text { bis zum } \\
\text { 1. Geburtstag }\end{array}$ & $\begin{array}{l}\text { bis zum } \\
\text { 3. Geburtstag }\end{array}$ & $\begin{array}{l}\text { bis zum } \\
\text { 10. Geburtstag }\end{array}$ & \begin{tabular}{l} 
alter als Jahre \\
\hline Verkürzung
\end{tabular} \\
\hline Varus & $\mathrm{cm}$ & $2 \mathrm{~cm}$ & $1 \mathrm{~cm}$ & $1 \mathrm{~cm}$ \\
\hline Valgus & $30^{\circ}$ & $25^{\circ}$ & $10^{\circ}$ & $5^{\circ}$ \\
\hline Antekurvation & $20^{\circ}$ & $15^{\circ}$ & $10^{\circ}$ & $5^{\circ}$ \\
\hline Rekurvation & $20^{\circ}$ & $20^{\circ}$ & $15^{\circ}$ & $5^{\circ}$ \\
\hline Rotation & $15^{\circ}$ & $10^{\circ}$ & $10^{\circ}$ & $5^{\circ}$ \\
\hline & $<10^{\circ}$ & $<10^{\circ}$ & 0 & 0
\end{tabular}

\subsection{Pädiatrische Frakturklassifikation für lange Röhrenknochen nach Slongo}

Angelehnt an die AO-Klassifikation nach Müller entwickelten die Arbeitsgruppen „AO Pediatric Classification Group“, „AO Pediatric Expert Group“ und „AO Clinical Investigation and Documentation Group" unter der Leitung von Theddy Slongo und Laurent Audigé im Jahr 2007 eine Klassifikation für Frakturen der langen Röhrenknochen im Wachstumsalter. Sie trägt den Titel „AO Pediatric Comprehensive Classification of Long-Bone Fractures (PCCF)".

Jede Fraktur erhält einen Zahlen-Buchstaben-Code, wobei die ersten vier Ziffern die Lokalisation, die weiteren zwei (eventuell drei) Ziffern die Morphologie der Fraktur beschreiben.

Die erste Ziffer gibt den oder die betroffenen Röhrenknochen an: 
2 -Radius/Ulna

3- Femur

4- Tibia/Fibula

An zweiter Stelle folgt die Angabe zur Höhe des betroffenen Knochensegments: 1=proximal, 2=diaphysär, 3=distal.

Für den Fall, dass von den paarigen Knochen Radius/Ulna und Tibia/Fibula nur einer

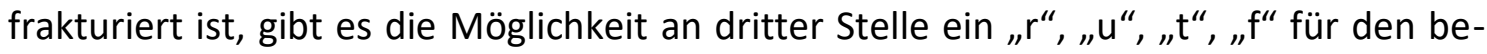
troffenen Knochen hinzuzufügen. Sind Humerus, Femur oder beide der paarigen Knochen betroffen, entfällt die dritte Stelle des Codes.

An vierter Stelle wird das Subsegment ausgewiesen, in dem das Frakturzentrum liegt:

E-Epiphyse

M-Metaphyse,

D- Diaphyse.

Im Gegensatz zum Knochensegment 2 enthalten die Knochensegmente 1 und 3 je zwei Subsegmente: Epi- und Metaphyse. Die Länge der Metaphyse wird am besten mit Hilfe von auf Folien vorgedruckten Quadraten ermittelt. Dabei muss ein Quadrat gewählt werden, dessen Quadratseite der weitesten Stelle der Wachstumsplatte entspricht. Sind Unterarm oder Unterschenkel gebrochen, so müssen die Epiphysenfugen beider Knochen zusammen abgemessen werden.

Im zweiten Teil und an fünfter Stelle des Codes wird eine Zahl von 1-9 verschiedenen kinderspezifischen Frakturmustern zugeordnet.

Die sechste Stelle im Code gibt Informationen über den Schweregrad, wobei lediglich zwischen „einfacher (=.1)“ und „komplexer (=.2)“ (mehr als zwei Fragmentstücke) Fraktur unterschieden wird.

Für Frakturen, die nicht nach den bisher genannten Ziffern eingeordnet werden können, sind Zusatz- und Ausnahme-Codes definiert worden, bspw. für Bandausrisse, suprakondyläre Humerusfrakturen, proximale Femurfrakturen etc.

Die in der vorliegenden Arbeit untersuchten Femurschaftfrakturen würden nach obiger Klassifikation z.B. den Code 32-D/4.1 zur Kennzeichnung einer einfachen Querfraktur erhalten (Slongo et al. 2010; Vogel 2012; Weinberg et al. 2010). 


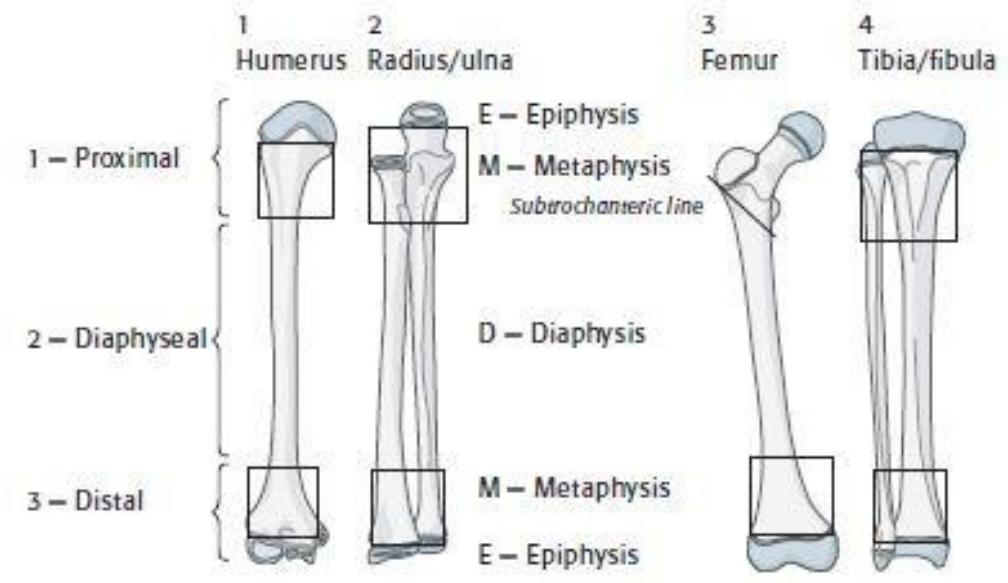

Abbildung 45: Einteilung und Nummerierung der Knochen nach der AO-PCCF

32-D Diaphyseal fractures

\begin{tabular}{l|ll|l|} 
Simple & Multifragmentary & Simple & Multifragmentary \\
32-D/4.1 & $32-\mathrm{D} / 4.2$ & $32-\mathrm{D} / 5.1$
\end{tabular}

33-M Distal metaphyseal fractures

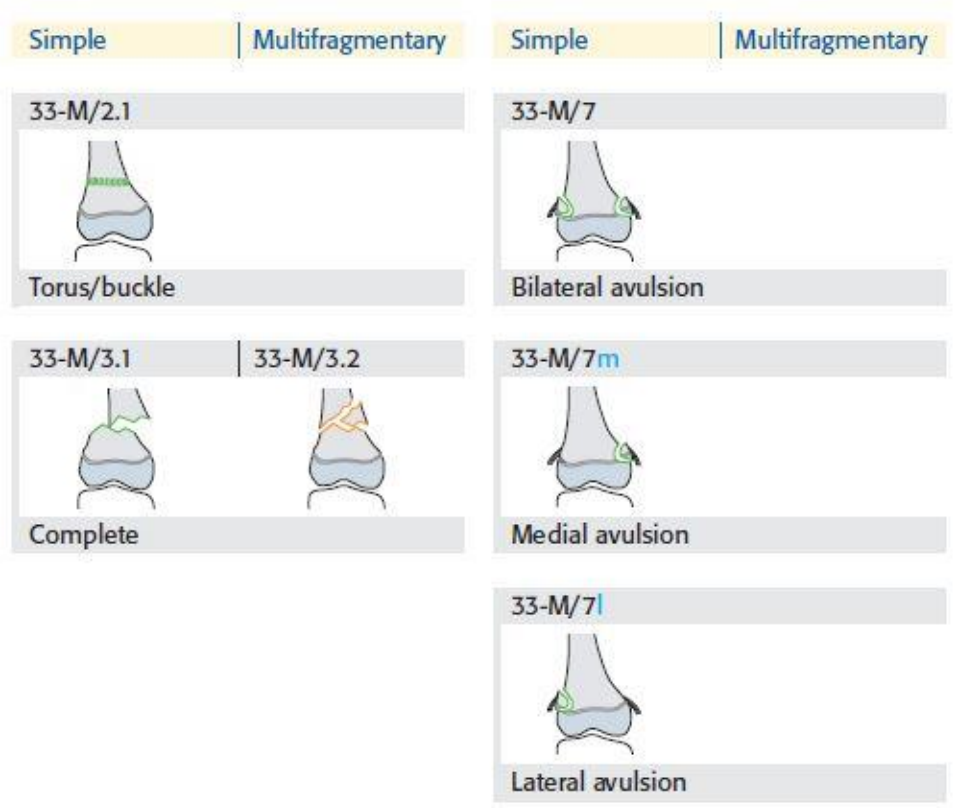

Abbildung 46: Einteilung von diaphysären Frakturen der AO-PCCF 


\subsection{Weiterführende Übersichten und Tabellen zu Kapitel 3}

Tabelle 15: Häufigkeiten des Unfallgeschehens je nach BMI der Patient(inn)en. (1 = starkes Untergewicht $<$ P3, 2 = Untergewicht P3 - P9.9, $3=$ Normalgewicht P10 - P89.9, $4=$ Übergewicht P90- P97, 5 = starkes Übergewicht > P97)

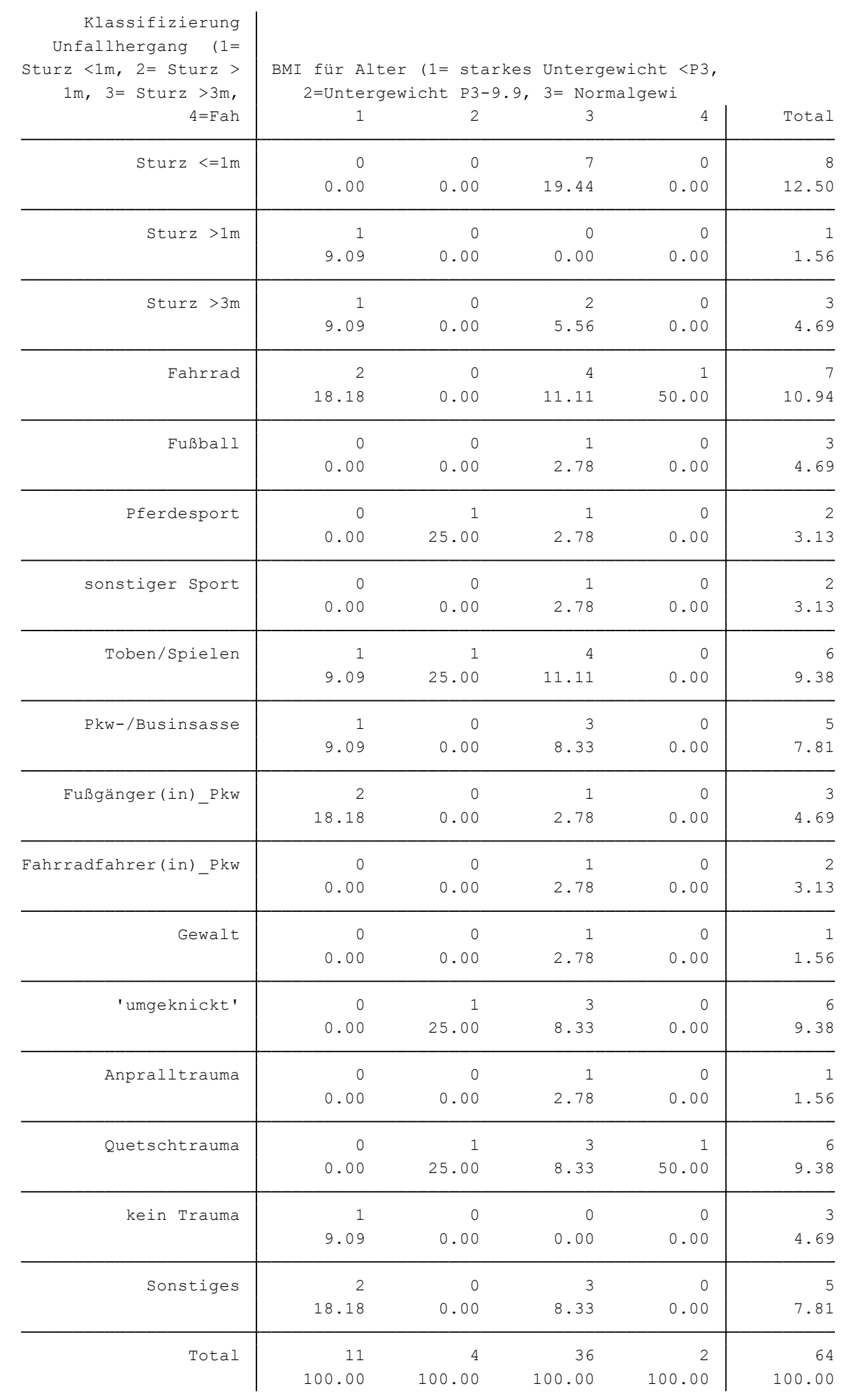




\begin{tabular}{|c|c|c|}
\hline $\begin{array}{r}\text { Klassifizierung } \\
\text { Unfallhergang }(1= \\
\text { Sturz <1m, 2= Sturz }> \\
1 \mathrm{~m}, 3=\text { Sturz }>3 \mathrm{~m}, \\
4=\text { Fah }\end{array}$ & $\begin{array}{c}\text { BMI für } \\
\text { Alter (1= } \\
\text { starkes } \\
\text { Untergewic } \\
\text { ht <P3, } \\
\text { 2=Untergew } \\
\text { icht } \\
\text { P3-9.9, } 3= \\
\text { Normalgewi } \\
5\end{array}$ & Total \\
\hline Sturz $<=1 \mathrm{~m}$ & $\begin{array}{r}1 \\
9.09\end{array}$ & $\begin{array}{r}8 \\
12.50\end{array}$ \\
\hline Sturz $>1 \mathrm{~m}$ & $\begin{array}{r}0 \\
0.00\end{array}$ & $\begin{array}{r}1 \\
1.56\end{array}$ \\
\hline Sturz $>3 m$ & $\begin{array}{r}0 \\
0.00\end{array}$ & $\begin{array}{r}3 \\
4.69\end{array}$ \\
\hline Fahrrad & $\begin{array}{r}0 \\
0.00\end{array}$ & $\begin{array}{r}7 \\
10.94\end{array}$ \\
\hline Fußball & $\begin{array}{r}2 \\
18.18\end{array}$ & $\begin{array}{r}3 \\
4.69\end{array}$ \\
\hline Pferdesport & $\begin{array}{r}0 \\
0.00\end{array}$ & $\begin{array}{r}2 \\
3.13\end{array}$ \\
\hline sonstiger Sport & $\begin{array}{r}1 \\
9.09\end{array}$ & $\begin{array}{r}2 \\
3.13\end{array}$ \\
\hline Toben/Spielen & $\begin{array}{r}0 \\
0.00\end{array}$ & $\begin{array}{r}6 \\
9.38\end{array}$ \\
\hline Pkw-/Businsasse & $\begin{array}{r}1 \\
9.09\end{array}$ & $\begin{array}{r}5 \\
7.81\end{array}$ \\
\hline Fußgänger(in)_Pkw & $\begin{array}{r}0 \\
0.00\end{array}$ & $\begin{array}{r}3 \\
4.69\end{array}$ \\
\hline Fahrradfahrer(in)_Pkw & $\begin{array}{r}1 \\
9.09\end{array}$ & $\begin{array}{r}2 \\
3.13\end{array}$ \\
\hline Gewalt & $\begin{array}{r}0 \\
0.00\end{array}$ & $\begin{array}{r}1 \\
1.56\end{array}$ \\
\hline 'umgeknickt' & $\begin{array}{r}2 \\
18.18\end{array}$ & $\begin{array}{r}6 \\
9.38\end{array}$ \\
\hline Anpralltrauma & $\begin{array}{r}0 \\
0.00\end{array}$ & $\begin{array}{r}1 \\
1.56\end{array}$ \\
\hline Quetschtrauma & $\begin{array}{r}1 \\
9.09\end{array}$ & $\begin{array}{r}6 \\
9.38\end{array}$ \\
\hline kein Trauma & $\begin{array}{r}2 \\
18.18\end{array}$ & $\begin{array}{r}3 \\
4.69\end{array}$ \\
\hline Sonstiges & $\begin{array}{r}0 \\
0.00\end{array}$ & $\begin{array}{r}5 \\
7.81\end{array}$ \\
\hline Total & $\begin{array}{r}11 \\
100.00\end{array}$ & $\begin{array}{r}64 \\
100.00\end{array}$ \\
\hline
\end{tabular}


Tabelle 16: Unfallgeschehen bei den unter Dreijährigen (1) im Vergleich zu den Drei- bis Sechzehnjährigen (0).

\begin{tabular}{|c|c|c|c|}
\hline $\begin{array}{r}\text { Klassifizierung } \\
\text { Unfallhergang }(1= \\
\text { Sturz <1m, } 2=\text { Sturz }> \\
1 \mathrm{~m}, 3=\text { Sturz }>3 \mathrm{~m}, \\
4=\text { Fah }\end{array}$ & $\begin{array}{l}j \\
0\end{array}$ & 1 & Total \\
\hline Sturz $<=1 \mathrm{~m}$ & $\begin{array}{r}7 \\
9.33\end{array}$ & $\begin{array}{r}4 \\
16.67\end{array}$ & $\begin{array}{r}11 \\
11.11\end{array}$ \\
\hline Sturz $>1 \mathrm{~m}$ & $\begin{array}{r}4 \\
5.33\end{array}$ & $\begin{array}{r}3 \\
12.50\end{array}$ & $\begin{array}{r}7 \\
7.07\end{array}$ \\
\hline Sturz $>3 m$ & $\begin{array}{r}4 \\
5.33\end{array}$ & $\begin{array}{r}0 \\
0.00\end{array}$ & $\begin{array}{r}4 \\
4.04\end{array}$ \\
\hline Fahrrad & $\begin{array}{r}9 \\
12.00\end{array}$ & $\begin{array}{r}0 \\
0.00\end{array}$ & $\begin{array}{r}9 \\
9.09\end{array}$ \\
\hline Fußball & $\begin{array}{r}5 \\
6.67\end{array}$ & $\begin{array}{r}1 \\
4.17\end{array}$ & $\begin{array}{r}6 \\
6.06\end{array}$ \\
\hline Pferdesport & $\begin{array}{r}2 \\
2.67\end{array}$ & $\begin{array}{r}0 \\
0.00\end{array}$ & $\begin{array}{r}2 \\
2.02\end{array}$ \\
\hline sonstiger sport & $\begin{array}{r}3 \\
4.00^{3}\end{array}$ & $\begin{array}{r}1 \\
4.17\end{array}$ & $\begin{array}{r}4 \\
4.04\end{array}$ \\
\hline Toben/Spielen & $\begin{array}{r}4 \\
5.33\end{array}$ & $\begin{array}{r}6 \\
25.00\end{array}$ & $\begin{array}{r}10 \\
10.10\end{array}$ \\
\hline Pkw-/Businsasse & $\begin{array}{r}7 \\
9.33\end{array}$ & $\begin{array}{r}1 \\
4.17\end{array}$ & $\begin{array}{r}8 \\
8.08\end{array}$ \\
\hline Fußgänger(in)_Pkw & $\begin{array}{r}6 \\
8.00\end{array}$ & $\begin{array}{r}0 \\
0.00\end{array}$ & $\begin{array}{r}6 \\
6.06\end{array}$ \\
\hline Fahrradfahrer(in)_Pkw & $\begin{array}{r}2 \\
2.67\end{array}$ & $\begin{array}{r}0 \\
0.00\end{array}$ & $\begin{array}{r}2 \\
2.02\end{array}$ \\
\hline Gewalt & $\begin{array}{r}0 \\
0.00\end{array}$ & $\begin{array}{r}1 \\
4.17\end{array}$ & $\begin{array}{r}1 \\
1.01\end{array}$ \\
\hline 'umgeknickt' & $\begin{array}{r}7 \\
9.33\end{array}$ & $\begin{array}{r}3 \\
12.50\end{array}$ & $\begin{array}{r}10 \\
10.10\end{array}$ \\
\hline Anpralltrauma & $\begin{array}{r}2 \\
2.67\end{array}$ & $\begin{array}{r}0 \\
0.00\end{array}$ & $\begin{array}{r}2 \\
2.02\end{array}$ \\
\hline Quetschtrauma & $\begin{array}{r}6 \\
8.00\end{array}$ & $\begin{array}{r}1 \\
4.17\end{array}$ & $\begin{array}{r}7 \\
7.07\end{array}$ \\
\hline kein Trauma & $\begin{array}{r}4 \\
5.33\end{array}$ & $\begin{array}{r}1 \\
4.17\end{array}$ & $\begin{array}{r}5 \\
5.05\end{array}$ \\
\hline Sonstiges & $\begin{array}{r}3 \\
4.00\end{array}$ & $\begin{array}{r}2 \\
8.33\end{array}$ & $\begin{array}{r}5 \\
5.05\end{array}$ \\
\hline Total & $\begin{array}{r}75 \\
100.00\end{array}$ & $\begin{array}{r}24 \\
100.00\end{array}$ & $\begin{array}{r}99 \\
100.00\end{array}$ \\
\hline
\end{tabular}


Tabelle 17: Unfallgeschehen bei den Drei- bis Achteinhalbjährigen (1) im Vergleich zu den unter Dreijährigen und über Achteinhalbjährigen (0)

\begin{tabular}{|c|c|c|c|}
\hline $\begin{array}{r}\text { Klassifizierung } \\
\text { Unfallhergang }(1= \\
\text { Sturz <1m, } 2=\text { Sturz }> \\
1 \mathrm{~m}, 3=\text { Sturz }>3 \mathrm{~m}, \\
4=\text { Fah }\end{array}$ & $\begin{array}{c}\text { Alter bei } \\
<8 \\
0\end{array}$ & $\begin{aligned}>=3 \& & \\
& 1\end{aligned}$ & Total \\
\hline Sturz $<=1 \mathrm{~m}$ & $\begin{array}{r}8 \\
13.79\end{array}$ & $\begin{array}{r}2 \\
8.33\end{array}$ & $\begin{array}{r}10 \\
12.20\end{array}$ \\
\hline Sturz $>1 \mathrm{~m}$ & $\begin{array}{r}3 \\
5.17\end{array}$ & $\begin{array}{r}1 \\
4.17\end{array}$ & $\begin{array}{r}4 \\
4.88\end{array}$ \\
\hline Sturz $>3 m$ & $\begin{array}{r}3 \\
5.17\end{array}$ & $\begin{array}{r}1 \\
4.17\end{array}$ & $\begin{array}{r}4 \\
4.88\end{array}$ \\
\hline Fahrrad & $\begin{array}{r}4 \\
6.90\end{array}$ & $\begin{array}{r}4 \\
16.67\end{array}$ & $\begin{array}{r}8 \\
9.76\end{array}$ \\
\hline Fußball & $\begin{array}{r}2 \\
3.45\end{array}$ & $\begin{array}{r}3 \\
12.50\end{array}$ & $\begin{array}{r}5 \\
6.10\end{array}$ \\
\hline Pferdesport & $\begin{array}{r}2 \\
3.45\end{array}$ & $\begin{array}{r}0 \\
0.00\end{array}$ & $\begin{array}{r}2 \\
2.44\end{array}$ \\
\hline sonstiger Sport & $\begin{array}{r}3 \\
5.17\end{array}$ & $\begin{array}{r}0 \\
0.00\end{array}$ & $\begin{array}{r}3 \\
3.66\end{array}$ \\
\hline Toben/Spielen & $\begin{array}{r}8 \\
13.79\end{array}$ & $\begin{array}{r}1 \\
4.17\end{array}$ & $\begin{array}{r}9 \\
10.98\end{array}$ \\
\hline $\mathrm{Pkw}-/$ Businsasse & $\begin{array}{r}5 \\
8.62\end{array}$ & $\begin{array}{r}2 \\
8.33\end{array}$ & $\begin{array}{r}7 \\
8.54\end{array}$ \\
\hline Fußgänger(in)_Pkw & $\begin{array}{r}5 \\
8.62\end{array}$ & $\begin{array}{r}0 \\
0.00\end{array}$ & $\begin{array}{r}5 \\
6.10\end{array}$ \\
\hline Fahrradfahrer(in)_Pkw & $\begin{array}{r}1 \\
1.72\end{array}$ & $\begin{array}{r}1 \\
4.17\end{array}$ & $\begin{array}{r}2 \\
2.44\end{array}$ \\
\hline Gewalt & $\begin{array}{r}1 \\
1.72\end{array}$ & $\begin{array}{r}0 \\
0.00\end{array}$ & $\begin{array}{r}1 \\
1.22\end{array}$ \\
\hline 'umgeknickt' & $\begin{array}{r}3 \\
5.17\end{array}$ & $\begin{array}{r}3 \\
12.50\end{array}$ & $\begin{array}{r}6 \\
7.32\end{array}$ \\
\hline Anpralltrauma & $\begin{array}{r}1 \\
1.72\end{array}$ & $\begin{array}{r}0 \\
0.00\end{array}$ & $\begin{array}{r}1 \\
1.22\end{array}$ \\
\hline Quetschtrauma & $\begin{array}{r}3 \\
5.17\end{array}$ & $\begin{array}{r}4 \\
16.67\end{array}$ & $\begin{array}{r}7 \\
8.54\end{array}$ \\
\hline kein Trauma & $\begin{array}{r}1 \\
1.72\end{array}$ & $\begin{array}{r}2 \\
8.33\end{array}$ & $\begin{array}{r}3 \\
3.66\end{array}$ \\
\hline Sonstiges & $\begin{array}{r}5 \\
8.62\end{array}$ & $\begin{array}{r}0 \\
0.00\end{array}$ & $\begin{array}{r}5 \\
6.10\end{array}$ \\
\hline Total & $\begin{array}{r}58 \\
100.00\end{array}$ & $\begin{array}{r}24 \\
100.00\end{array}$ & $\begin{array}{r}82 \\
100.00\end{array}$ \\
\hline
\end{tabular}


Tabelle 18: Unfallgeschehen bei den über Achteinhalbjährigen (1) im Vergleich zu den unter Achteinhalbjährigen (0)

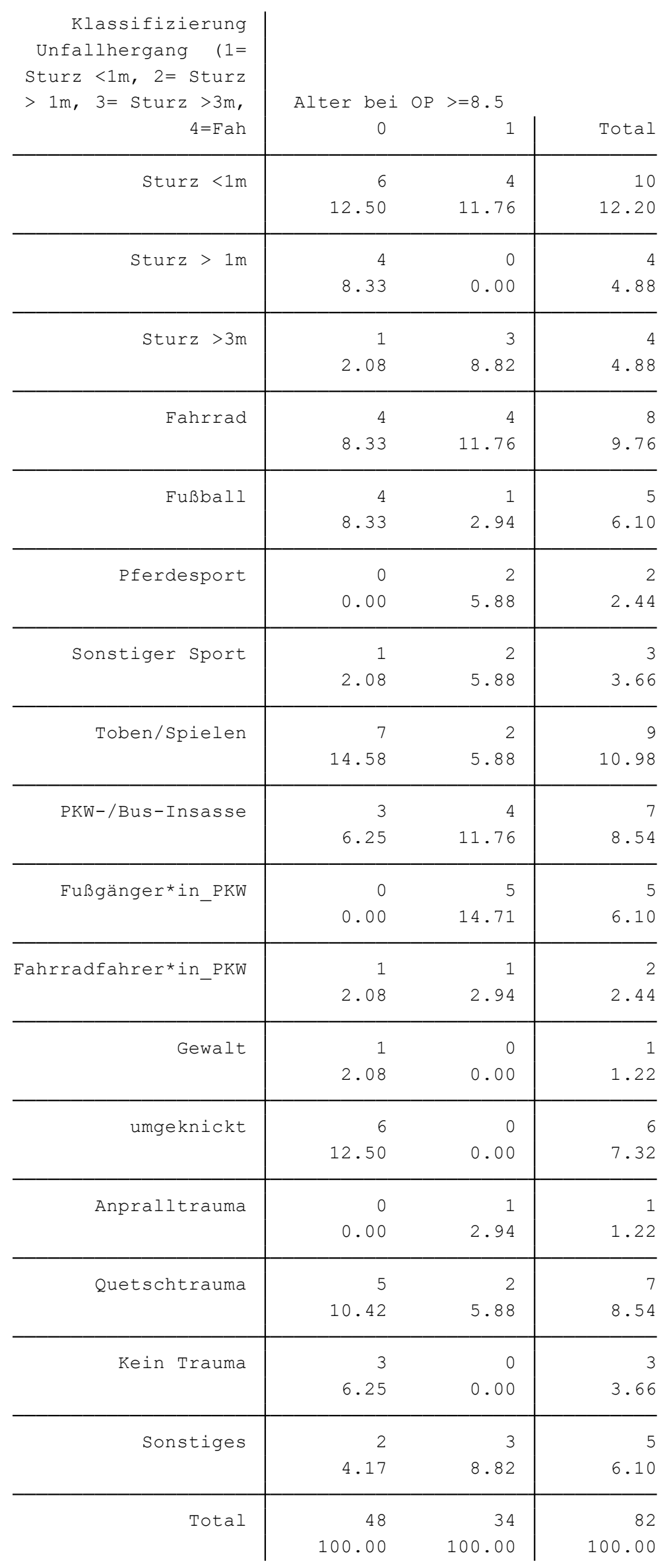


Tabelle 19: Frakturtypen und jeweilige Unfallgeschehen: Beispielsweise ereigneten sich $20 \%$ der Schrägfrakturen bei einem Sturz aus einer Höhe von unter einem Meter. Frakturtyp $1=$ Querfraktur, $2=$ Schrägfraktur, $3=$ Spiralfraktur. $4=$ Trümmerfraktur, $5=$ Mehrfragmentfraktur, $6=$ „,Femurschaftfraktur" ${ }^{\prime}, 7=$ nicht zuzuordnen

\begin{tabular}{|c|c|c|c|c|c|}
\hline $\begin{array}{r}\text { Klassifizierung } \\
\text { Unfallhergang }(1= \\
\text { Sturz }<1 \mathrm{~m}, 2=\text { Sturz }> \\
1 \mathrm{~m}, 3=\text { Sturz }>3 \mathrm{~m}, \\
4=\mathrm{Fah}\end{array}$ & $\begin{array}{r}\text { Frakturty } \\
3=\text { Spir } \\
1\end{array}$ & $\begin{array}{l}=\text { Querfr } \\
\text { aktur, } \\
\\
2\end{array}$ & $\begin{array}{r}\quad 2=\mathrm{Sch} \\
3\end{array}$ & $\begin{array}{l}\text { fraktur, } \\
\text { r, } 5 \\
\\
\end{array}$ & Total \\
\hline Sturz $<=1 \mathrm{~m}$ & $\begin{array}{r}1 \\
6.67\end{array}$ & $\begin{array}{r}3 \\
20.00\end{array}$ & $\begin{array}{r}4 \\
12.50\end{array}$ & $\begin{array}{r}0 \\
0.00\end{array}$ & $\begin{array}{r}11 \\
11.11\end{array}$ \\
\hline Sturz $>1 \mathrm{~m}$ & $\begin{array}{r}2 \\
13.33\end{array}$ & $\begin{array}{r}0 \\
0.00\end{array}$ & $\begin{array}{r}2 \\
6.25\end{array}$ & $\begin{array}{r}0 \\
0.00\end{array}$ & $\begin{array}{r}7 \\
7.07\end{array}$ \\
\hline Sturz $>3 m$ & $\begin{array}{r}1 \\
6.67\end{array}$ & $\begin{array}{r}0 \\
0.00\end{array}$ & $\begin{array}{r}1 \\
3.13\end{array}$ & $\begin{array}{r}0 \\
0.00\end{array}$ & $\begin{array}{r}4 \\
4.04\end{array}$ \\
\hline Fahrrad & $\begin{array}{r}2 \\
13.33\end{array}$ & $\begin{array}{r}0 \\
0.00\end{array}$ & $\begin{array}{r}2 \\
6.25\end{array}$ & $\begin{array}{r}0 \\
0.00\end{array}$ & $\begin{array}{r}9 \\
9.09\end{array}$ \\
\hline Fußball & $\begin{array}{r}0 \\
0.00\end{array}$ & $\begin{array}{r}1 \\
6.67\end{array}$ & $\begin{array}{r}4 \\
12.50\end{array}$ & $\begin{array}{r}0 \\
0.00\end{array}$ & $\begin{array}{r}6 \\
6.06\end{array}$ \\
\hline Pferdesport & $\begin{array}{r}2 \\
13.33\end{array}$ & $\begin{array}{r}0 \\
0.00\end{array}$ & $\begin{array}{r}0 \\
0.00\end{array}$ & $\begin{array}{r}0 \\
0.00\end{array}$ & $\begin{array}{r}2 \\
2.02\end{array}$ \\
\hline sonstiger sport & $\begin{array}{r}0 \\
0.00\end{array}$ & $\begin{array}{r}1 \\
6.67\end{array}$ & $\begin{array}{r}2 \\
6.25\end{array}$ & $\begin{array}{r}0 \\
0.00\end{array}$ & $\begin{array}{r}4 \\
4.04\end{array}$ \\
\hline Toben/Spielen & $\begin{array}{r}3 \\
20.00\end{array}$ & $\begin{array}{r}2 \\
13.33\end{array}$ & $\begin{array}{r}2 \\
6.25\end{array}$ & $\begin{array}{r}0 \\
0.00\end{array}$ & $\begin{array}{r}10 \\
10.10\end{array}$ \\
\hline PKW-/Bus-Insasse & $\begin{array}{r}2 \\
13.33\end{array}$ & $\begin{array}{r}0 \\
0.00\end{array}$ & $\begin{array}{r}0 \\
0.00\end{array}$ & $\begin{array}{r}1 \\
100.00\end{array}$ & $\begin{array}{r}8 \\
8.08\end{array}$ \\
\hline Fußgänger(in)_PKW & $\begin{array}{r}0 \\
0.00\end{array}$ & $\begin{array}{r}1 \\
6.67\end{array}$ & $\begin{array}{r}2 \\
6.25\end{array}$ & $\begin{array}{r}0 \\
0.00\end{array}$ & $\begin{array}{r}6 \\
6.06\end{array}$ \\
\hline Fahrradfahrer(in)_PKW & $\begin{array}{r}0 \\
0.00\end{array}$ & $\begin{array}{r}1 \\
6.67\end{array}$ & $\begin{array}{r}0 \\
0.00\end{array}$ & $\begin{array}{r}0 \\
0.00\end{array}$ & $\begin{array}{r}2 \\
2.02\end{array}$ \\
\hline Gewalt & $\begin{array}{r}0 \\
0.00\end{array}$ & $\begin{array}{r}1 \\
6.67\end{array}$ & $\begin{array}{r}0 \\
0.00\end{array}$ & $\begin{array}{r}0 \\
0.00\end{array}$ & $\begin{array}{r}1 \\
1.01\end{array}$ \\
\hline umgeknickt & $\begin{array}{r}0 \\
0.00\end{array}$ & $\begin{array}{r}3 \\
20.00\end{array}$ & $\begin{array}{r}5 \\
15.63\end{array}$ & $\begin{array}{r}0 \\
0.00\end{array}$ & $\begin{array}{r}10 \\
10.10\end{array}$ \\
\hline Anpralltrauma & $\begin{array}{r}0 \\
0.00\end{array}$ & $\begin{array}{r}0 \\
0.00\end{array}$ & $\begin{array}{r}1 \\
3.13\end{array}$ & $\begin{array}{r}0 \\
0.00\end{array}$ & $\begin{array}{r}2 \\
2.02\end{array}$ \\
\hline Quetschtrauma & $\begin{array}{r}2 \\
13.33\end{array}$ & $\begin{array}{r}0 \\
0.00\end{array}$ & $\begin{array}{r}4 \\
12.50\end{array}$ & $\begin{array}{r}0 \\
0.00\end{array}$ & $\begin{array}{r}7 \\
7.07\end{array}$ \\
\hline kein Trauma & $\begin{array}{r}0 \\
0.00\end{array}$ & $\begin{array}{r}1 \\
6.67\end{array}$ & $\begin{array}{r}2 \\
6.25\end{array}$ & $\begin{array}{r}0 \\
0.00\end{array}$ & $\begin{array}{r}5 \\
5.05\end{array}$ \\
\hline Sonstiges & $\begin{array}{r}0 \\
0.00\end{array}$ & $\begin{array}{r}1 \\
6.67\end{array}$ & $\begin{array}{r}1 \\
3.13\end{array}$ & $\begin{array}{r}0 \\
0.00\end{array}$ & $\begin{array}{r}5 \\
5.05\end{array}$ \\
\hline Total & $\begin{array}{r}15 \\
100.00\end{array}$ & $\begin{array}{r}15 \\
100.00\end{array}$ & $\begin{array}{r}32 \\
100.00\end{array}$ & $\begin{array}{r}1 \\
100.00\end{array}$ & $\begin{array}{r}99 \\
100.00\end{array}$ \\
\hline
\end{tabular}




\begin{tabular}{|c|c|c|c|c|}
\hline $\begin{array}{r}\text { Klassifizierung } \\
\text { Unfallhergang }(1= \\
\text { Sturz <1m, } 2=\text { Sturz }> \\
1 \mathrm{~m}, 3=\text { Sturz }>3 \mathrm{~m}, \\
4=\text { Fah }\end{array}$ & $\begin{array}{r}\text { Fraktur } \\
2= \\
3= \\
4=\operatorname{Tr} \\
5\end{array}$ & $\begin{aligned} & (1 \text { =Quer } \\
\text { ägfrakt } & \text { alfrakt } \\
\text { rfraktu } & 6\end{aligned}$ & $\begin{array}{l}\text { ktur, } \\
5\end{array}$ & Total \\
\hline Sturz $<=1 \mathrm{~m}$ & $\begin{array}{r}0 \\
0.00\end{array}$ & $\begin{array}{r}3 \\
20.00\end{array}$ & $\begin{array}{r}0 \\
0.00\end{array}$ & $\begin{array}{r}11 \\
11.11\end{array}$ \\
\hline Sturz $>1 \mathrm{~m}$ & $\begin{array}{r}0 \\
0.00\end{array}$ & $\begin{array}{r}1 \\
6.67\end{array}$ & $\begin{array}{r}2 \\
13.33\end{array}$ & $\begin{array}{r}7 \\
7.07\end{array}$ \\
\hline Sturz $>3 m$ & $\begin{array}{r}0 \\
0.00\end{array}$ & $\begin{array}{r}2 \\
13.33\end{array}$ & $\begin{array}{r}0 \\
0.00\end{array}$ & $\begin{array}{r}4 \\
4.04\end{array}$ \\
\hline Fahrrad & $\begin{array}{r}1 \\
16.67\end{array}$ & $\begin{array}{r}2 \\
13.33\end{array}$ & $\begin{array}{r}2 \\
13.33\end{array}$ & $\begin{array}{r}9 \\
9.09\end{array}$ \\
\hline Fußball & $\begin{array}{r}0 \\
0.00\end{array}$ & $\begin{array}{r}0 \\
0.00\end{array}$ & $\begin{array}{r}1 \\
6.67\end{array}$ & $\begin{array}{r}6 \\
6.06\end{array}$ \\
\hline Pferdesport & $\begin{array}{r}0 \\
0.00\end{array}$ & $\begin{array}{r}0 \\
0.00\end{array}$ & $\begin{array}{r}0 \\
0.00\end{array}$ & $\begin{array}{r}2 \\
2.02\end{array}$ \\
\hline sonstiger sport & $\begin{array}{r}0 \\
0.00\end{array}$ & $\begin{array}{r}0 \\
0.00\end{array}$ & $\begin{array}{r}1 \\
6.67\end{array}$ & $\begin{array}{r}4 \\
4.04\end{array}$ \\
\hline Toben/Spielen & $\begin{array}{r}1 \\
16.67\end{array}$ & $\begin{array}{r}1 \\
6.67\end{array}$ & $\begin{array}{r}1 \\
6.67\end{array}$ & $\begin{array}{r}10 \\
10.10\end{array}$ \\
\hline PKW-/Bus-Insasse & $\begin{array}{r}3 \\
50.00\end{array}$ & $\begin{array}{r}0 \\
0.00\end{array}$ & $\begin{array}{r}2 \\
13.33\end{array}$ & $\begin{array}{r}8 \\
8.08\end{array}$ \\
\hline Fußgänger(in)_PKW & $\begin{array}{r}1 \\
16.67\end{array}$ & $\begin{array}{r}1 \\
6.67\end{array}$ & $\begin{array}{r}1 \\
6.67\end{array}$ & $\begin{array}{r}6 \\
6.06\end{array}$ \\
\hline Fahrradfahrer(in)_PKW & $\begin{array}{r}0 \\
0.00\end{array}$ & $\begin{array}{r}1 \\
6.67\end{array}$ & $\begin{array}{r}0 \\
0.00\end{array}$ & $\begin{array}{r}2 \\
2.02\end{array}$ \\
\hline Gewalt & $\begin{array}{r}0 \\
0.00\end{array}$ & $\begin{array}{r}0 \\
0.00\end{array}$ & $\begin{array}{r}0 \\
0.00\end{array}$ & $\begin{array}{r}1 \\
1.01\end{array}$ \\
\hline umgeknickt & $\begin{array}{r}0 \\
0.00\end{array}$ & $\begin{array}{r}1 \\
6.67\end{array}$ & $\begin{array}{r}1 \\
6.67\end{array}$ & $\begin{array}{r}10 \\
10.10\end{array}$ \\
\hline Anpralltrauma & $\begin{array}{r}0 \\
0.00\end{array}$ & $\begin{array}{r}0 \\
0.00\end{array}$ & $\begin{array}{r}1 \\
6.67\end{array}$ & $\begin{array}{r}2 \\
2.02\end{array}$ \\
\hline Quetschtrauma & $\begin{array}{r}0 \\
0.00\end{array}$ & $\begin{array}{r}0 \\
0.00\end{array}$ & $\begin{array}{r}1 \\
6.67\end{array}$ & $\begin{array}{r}7 \\
7.07\end{array}$ \\
\hline kein Trauma & $\begin{array}{r}0 \\
0.00\end{array}$ & $\begin{array}{r}2 \\
13.33\end{array}$ & $\begin{array}{r}0 \\
0.00\end{array}$ & $\begin{array}{r}5 \\
5.05\end{array}$ \\
\hline Sonstiges & $\begin{array}{r}0 \\
0.00\end{array}$ & $\begin{array}{r}1 \\
6.67\end{array}$ & $\begin{array}{r}2 \\
13.33\end{array}$ & $\begin{array}{r}5 \\
5.05\end{array}$ \\
\hline Total & $\begin{array}{r}6 \\
100.00\end{array}$ & $\begin{array}{r}15 \\
100.00\end{array}$ & $\begin{array}{r}15 \\
100.00\end{array}$ & $\begin{array}{r}99 \\
100.00\end{array}$ \\
\hline
\end{tabular}


Tabelle 20: Häufigkeiten der Frakturtypen bei den jeweiligen Unfallgeschehen: Beispielsweise erlitten 36\% der Patient(inn)en, die aus einer Höhe von unter einem Meter stürzten, eine Spiralfraktur. Frakturtyp 1 = Querfraktur, $2=$ Schrägfraktur, $3=$ Spiralfraktur, $4=$ Trümmerfraktur, 5 = Mehrfragmentfraktur, $6=$ „Femurschaftfraktur" 7 = nicht zuzuordnen.

\begin{tabular}{|c|c|c|c|c|c|}
\hline $\begin{array}{r}\text { Klassifizierung } \\
\text { Unfallhergang }(1= \\
\text { Sturz <1m, 2= Sturz > } \\
1 \mathrm{~m}, 3=\text { Sturz }>3 \mathrm{~m}, \\
4=\text { Fah }\end{array}$ & \multicolumn{4}{|c|}{$\begin{array}{c}\text { Frakturtyp (1=Querfraktur, } 2=\text { Schrägfraktur, } \\
\text { 3=Spiralfraktur, } 4=\text { Trümmerfraktur, } 5\end{array}$} & Total \\
\hline \multirow[t]{2}{*}{ Sturz $<=1 \mathrm{~m}$} & 1 & 3 & 4 & 0 & 11 \\
\hline & 9.09 & 27.27 & 36.36 & 0.00 & 100.00 \\
\hline \multirow[t]{2}{*}{ Stur $z>1 \mathrm{~m}$} & 2 & 0 & 2 & 0 & 7 \\
\hline & 28.57 & 0.00 & 28.57 & 0.00 & 100.00 \\
\hline \multirow[t]{2}{*}{ Sturz $>3 \mathrm{~m}$} & 1 & 0 & 1 & 0 & 4 \\
\hline & 25.00 & 0.00 & 25.00 & 0.00 & 100.00 \\
\hline \multirow[t]{2}{*}{ Fahrrad } & 2 & 0 & 2 & 0 & 9 \\
\hline & 22.22 & 0.00 & 22.22 & 0.00 & 100.00 \\
\hline \multirow[t]{2}{*}{ Fußball } & 0 & 1 & 4 & 0 & 6 \\
\hline & 0.00 & 16.67 & 66.67 & 0.00 & 100.00 \\
\hline \multirow[t]{2}{*}{ Pferdesport } & 2 & 0 & 0 & 0 & 2 \\
\hline & 100.00 & 0.00 & 0.00 & 0.00 & 100.00 \\
\hline \multirow[t]{2}{*}{ sonstiger sport } & 0 & 1 & 2 & 0 & 4 \\
\hline & 0.00 & 25.00 & 50.00 & 0.00 & 100.00 \\
\hline \multirow[t]{2}{*}{ Toben/Spielen } & 3 & 2 & 2 & 0 & 10 \\
\hline & 30.00 & 20.00 & 20.00 & 0.00 & 100.00 \\
\hline \multirow[t]{2}{*}{ PKW-/Bus-Insasse } & 2 & 0 & 0 & 1 & 8 \\
\hline & 25.00 & 0.00 & 0.00 & 12.50 & 100.00 \\
\hline \multirow[t]{2}{*}{ Fußgänger(in)_PKW } & 0 & 1 & 2 & 0 & 6 \\
\hline & 0.00 & 16.67 & 33.33 & 0.00 & 100.00 \\
\hline \multirow{2}{*}{ Fahrradfahrer(in)_PKW } & 0 & 1 & 0 & 0 & 2 \\
\hline & 0.00 & 50.00 & 0.00 & 0.00 & 100.00 \\
\hline \multirow[t]{2}{*}{ Gewalt } & 0 & 1 & 0 & 0 & 1 \\
\hline & 0.00 & 100.00 & 0.00 & 0.00 & 100.00 \\
\hline \multirow[t]{2}{*}{ umge knickt } & 0 & 3 & 5 & 0 & 10 \\
\hline & 0.00 & 30.00 & 50.00 & 0.00 & 100.00 \\
\hline \multirow[t]{2}{*}{ Anprall trauma } & 0 & 0 & 1 & 0 & 2 \\
\hline & 0.00 & 0.00 & 50.00 & 0.00 & 100.00 \\
\hline \multirow[t]{2}{*}{ Quetschtrauma } & 2 & 0 & 4 & 0 & 7 \\
\hline & 28.57 & 0.00 & 57.14 & 0.00 & 100.00 \\
\hline \multirow[t]{2}{*}{ kein Trauma } & 0 & 1 & 2 & 0 & 5 \\
\hline & 0.00 & 20.00 & 40.00 & 0.00 & 100.00 \\
\hline \multirow[t]{2}{*}{ Sonstiges } & 0 & 1 & 1 & 0 & 5 \\
\hline & 0.00 & 20.00 & 20.00 & 0.00 & 100.00 \\
\hline \multirow[t]{2}{*}{ Total } & 15 & 15 & 32 & 1 & 99 \\
\hline & 15.15 & 15.15 & 32.32 & 1.01 & 100.00 \\
\hline
\end{tabular}




\begin{tabular}{|c|c|c|c|c|}
\hline \multirow[t]{2}{*}{$\begin{array}{r}\text { Klassifizierung } \\
\text { Unfallhergang }(1= \\
\text { Sturz <1m, 2= Sturz > } \\
1 \mathrm{~m}, 3=\text { Sturz }>3 \mathrm{~m}, \\
4=\text { Fah }\end{array}$} & \multicolumn{3}{|c|}{$\begin{array}{c}\text { Frakturtyp (1=Querfraktur, } \\
2=\text { Schrägfraktur, }\end{array}$} & \multirow[b]{2}{*}{ Total } \\
\hline & 5 & 6 & 7 & \\
\hline \multirow[t]{2}{*}{ Sturz $<=1 \mathrm{~m}$} & 0 & 3 & 0 & \\
\hline & 0.00 & 27.27 & 0.00 & 100.00 \\
\hline \multirow[t]{2}{*}{ Sturz $>1 \mathrm{~m}$} & 0 & 1 & 2 & \\
\hline & 0.00 & 14.29 & 28.57 & 100.00 \\
\hline \multirow[t]{2}{*}{ Sturz $>3 m$} & 0 & 2 & 0 & \\
\hline & 0.00 & 50.00 & 0.00 & 100.00 \\
\hline \multirow[t]{2}{*}{ Fahrrad } & 1 & 2 & 2 & \\
\hline & 11.11 & 22.22 & 22.22 & 100.00 \\
\hline \multirow[t]{2}{*}{ Fußball } & 0 & 0 & 1 & \\
\hline & 0.00 & 0.00 & 16.67 & 100.00 \\
\hline \multirow[t]{2}{*}{ Pferdesport } & 0 & 0 & 0 & \\
\hline & 0.00 & 0.00 & 0.00 & 100.00 \\
\hline \multirow[t]{2}{*}{ sonstiger sport } & 0 & 0 & 1 & \\
\hline & 0.00 & 0.00 & 25.00 & 100.00 \\
\hline \multirow[t]{2}{*}{ Toben/Spielen } & 1 & 1 & 1 & \\
\hline & 10.00 & 10.00 & 10.00 & 100.00 \\
\hline \multirow[t]{2}{*}{ PKW-/Bus-Insasse } & 3 & 0 & 2 & \\
\hline & 37.50 & 0.00 & 25.00 & 100.00 \\
\hline \multirow[t]{2}{*}{ Fußgänger(in)_PKW } & 1 & 1 & 1 & \\
\hline & 16.67 & 16.67 & 16.67 & 100.00 \\
\hline \multirow[t]{2}{*}{ Fahrradfahrer(in)_PKW } & 0 & 1 & 0 & \\
\hline & 0.00 & 50.00 & 0.00 & 100.00 \\
\hline \multirow[t]{2}{*}{ Gewalt } & 0 & 0 & 0 & \\
\hline & 0.00 & 0.00 & 0.00 & 100.00 \\
\hline \multirow[t]{2}{*}{ umgeknickt } & 0 & 1 & 1 & \\
\hline & 0.00 & 10.00 & 10.00 & 100.00 \\
\hline \multirow[t]{2}{*}{ Anpralltrauma } & 0 & 0 & 1 & \\
\hline & 0.00 & 0.00 & 50.00 & 100.00 \\
\hline \multirow[t]{2}{*}{ Quetschtrauma } & 0 & 0 & 1 & \\
\hline & 0.00 & 0.00 & 14.29 & 100.00 \\
\hline \multirow[t]{2}{*}{ kein Trauma } & 0 & 2 & 0 & \\
\hline & 0.00 & 40.00 & 0.00 & 100.00 \\
\hline \multirow[t]{2}{*}{ Sonstiges } & 0 & 1 & 2 & \\
\hline & 0.00 & 20.00 & 40.00 & 100.00 \\
\hline \multirow[t]{2}{*}{ Total } & 6 & 15 & 15 & \\
\hline & 6.06 & 15.15 & 15.15 & 100.00 \\
\hline
\end{tabular}


Tabelle 21: Art der Versorgung der jeweiligen Frakturtypen von 98 Patient(inn)en. Beispiel 1: 15 Patient(inn)en erlitten eine Querfraktur. In zehn Fällen wurden diese mit ESINs versorgt, einmal mit einer Overhead-Extension therapiert und viermal wurde eine Platte eingesetzt. Beispiel 2: Von 62 Patient(inn)en, denen ESINs implantiert worden waren, hatten die meisten, nämlich 22 Patient(inn)en, eine Spiralfraktur erlitten. Weitere, jeweils zehn, Patient(inn)en hatten eine Schräg- oder Querfraktur.

\begin{tabular}{r|rrrr|r} 
& \multicolumn{4}{|c}{ Art der Versorgung: } & initiales Material \\
Frakturtyp & ESIN & Fixateur & Gips & K-Draht & Total \\
\hline Querfraktur & 10 & 0 & 0 & 0 & 15 \\
& 66.67 & 0.00 & 0.00 & 0.00 & 100.00 \\
\hline Schrägfraktur & 10 & 0 & 0 & 1 & 15 \\
& 66.67 & 0.00 & 0.00 & 6.67 & 100.00 \\
\hline Spiralfraktur & 22 & 0 & 1 & 0 & 31 \\
& 70.97 & 0.00 & 3.23 & 0.00 & 100.00 \\
\hline Trümmerfraktur & 0 & 1 & 0 & 0 & 1 \\
& 0.00 & 100.00 & 0.00 & 0.00 & 100.00 \\
\hline nur Mehrfragmentfrakt & 2 & 1 & 0 & 0 & 7 \\
& 28.57 & 14.29 & 0.00 & 0.00 & 100.00 \\
\hline nur Femurschaftfraktu & 14 & 0 & 1 & 0 & 15 \\
& 93.33 & 0.00 & 6.67 & 0.00 & 100.00 \\
\hline nur Femurfraktur & 4 & 1 & 5 & 2 & 14 \\
& 28.57 & 7.14 & 35.71 & 14.29 & 100.00 \\
\hline \multirow{2}{*}{ Total } & 63.27 & 3.06 & 7.14 & 3.06 & 100.00
\end{tabular}




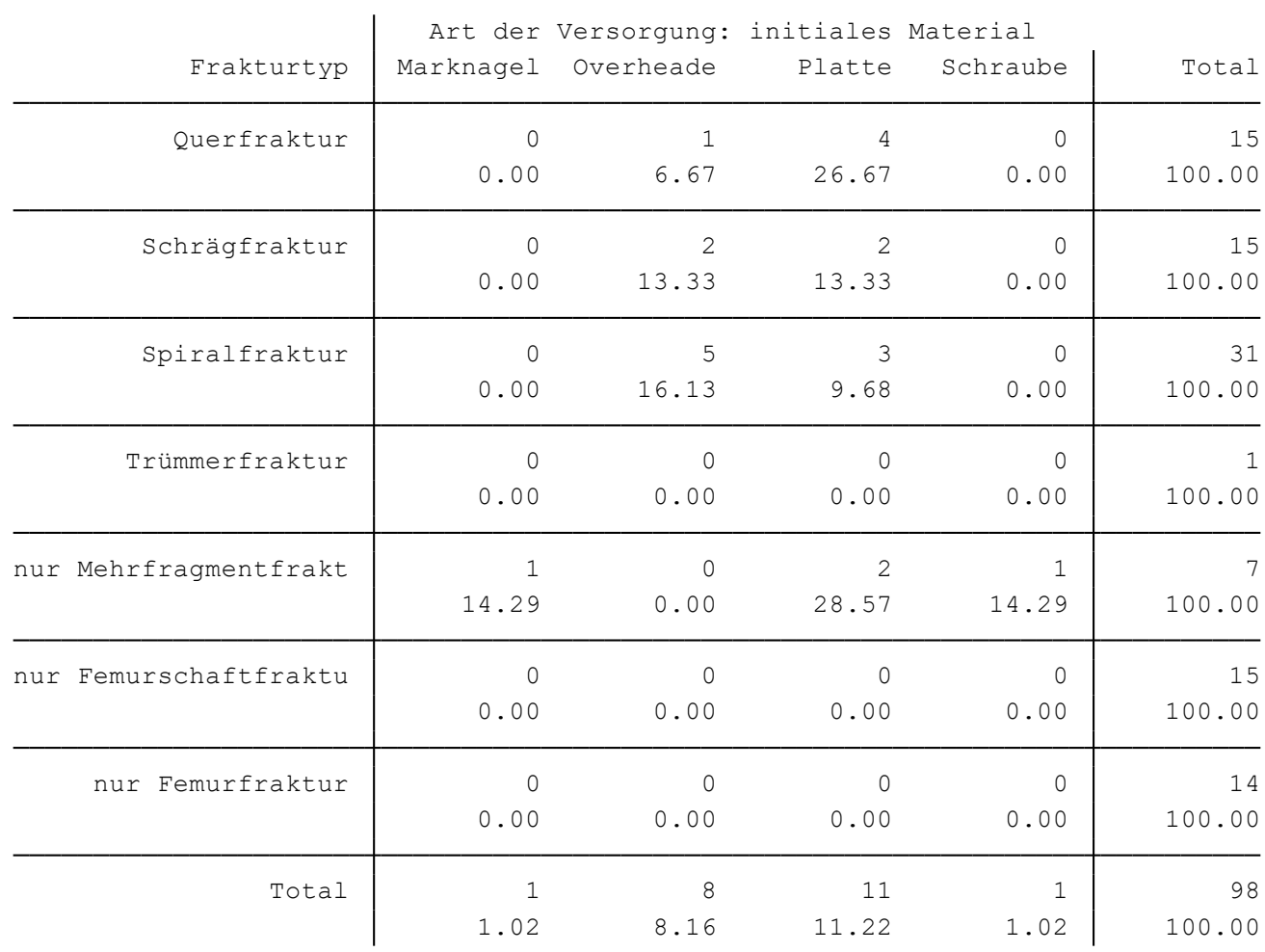

\begin{tabular}{|c|c|c|}
\hline Frakturtyp & $\begin{array}{c}\text { Art der } \\
\text { Versorgung } \\
: \\
\text { initiales } \\
\text { Material } \\
\text { Sonstiges }\end{array}$ & Total \\
\hline Querfraktur & $\begin{array}{r}0 \\
0.00\end{array}$ & $\begin{array}{r}15 \\
100.00\end{array}$ \\
\hline Schrägfraktur & $\begin{array}{r}0 \\
0.00\end{array}$ & $\begin{array}{r}15 \\
100.00\end{array}$ \\
\hline spiralfraktur & $\begin{array}{r}0 \\
0.00\end{array}$ & $\begin{array}{r}31 \\
100.00\end{array}$ \\
\hline Trümmerfraktur & $\begin{array}{r}0 \\
0.00\end{array}$ & $100.00^{1}$ \\
\hline nur Mehrfragmentfrakt & $\begin{array}{r}0 \\
0.00\end{array}$ & $\begin{array}{r}7 \\
100.00\end{array}$ \\
\hline nur Femurschaftfraktu & $\begin{array}{r}0 \\
0.00\end{array}$ & $\begin{array}{r}15 \\
100.00\end{array}$ \\
\hline nur Femurfraktur & $\begin{array}{r}2 \\
14.29\end{array}$ & $\begin{array}{r}14 \\
100.00\end{array}$ \\
\hline Total & $\begin{array}{r}2 \\
2.04\end{array}$ & $\begin{array}{r}98 \\
100.00\end{array}$ \\
\hline
\end{tabular}


Tabelle 22: Multivariablen-Regression von Komplikationen und Frakturtyp (Querfraktur als Vergleichsgruppe) sowie von Komplikationen und Art der Versorgung (ESIN als Vergleichsgruppe). Keine signifikanten Zusammenhänge feststellbar.

\begin{tabular}{|c|c|c|c|}
\hline Source & SS & $d f$ & MS \\
\hline Model & 1.04963258 & 14 & .074973756 \\
\hline Residual & 16.0115919 & 83 & .192910746 \\
\hline Total & 17.0612245 & 97 & .175888912 \\
\hline
\end{tabular}

$\begin{array}{llr}\text { Number of obs } & = & 98 \\ \text { F (14, 83) } & = & 0.39 \\ \text { Prob }>\text { F } & =0.9747 \\ \text { R-squared } & =0.0615 \\ \text { Adj R-squared } & = & -0.0968 \\ \text { Root MSE } & = & .43922\end{array}$

\begin{tabular}{|c|c|c|c|c|c|c|}
\hline Komplikation e & Coef. & Std. Err. & $t$ & $P>|t|$ & [95\% Conf. & Interval] \\
\hline \multicolumn{7}{|l|}{ Frakturtyp } \\
\hline Schrägfraktur & -.0378697 & .1628967 & -0.23 & 0.817 & -.3618647 & .2861252 \\
\hline Spiralfraktur & .0159906 & .1411984 & 0.11 & 0.910 & -.2648474 & .2968286 \\
\hline Trümmerfrak r & -.07076 & .5676609 & -0.12 & 0.901 & -1.199815 & 1.058295 \\
\hline nur Mehrfra.. & -.0544266 & .2385811 & -0.23 & 0.820 & -.5289549 & .4201017 \\
\hline nur Femursc.. & .111219 & .1665988 & 0.67 & 0.506 & -.2201394 & .4425773 \\
\hline nur Femurfr.. & -.0870934 & .2093205 & -0.42 & 0.678 & -.5034236 & .3292367 \\
\hline \multicolumn{7}{|l|}{ ArtderVersor g } \\
\hline Fixateur ex.. & -.1538701 & .343434 & -0.45 & 0.655 & -.8369465 & .5292064 \\
\hline Gips & -.0377362 & .2092627 & -0.18 & 0.857 & -.4539512 & .3784789 \\
\hline K-Draht & -.1539446 & .2828322 & -0.54 & 0.588 & -.7164864 & .4085973 \\
\hline Marknagel & -.1702035 & .4908041 & -0.35 & 0.730 & -1.146393 & .8059861 \\
\hline Overheadext $\sim \mathrm{n}$ & .0248432 & .1694775 & 0.15 & 0.884 & -.3122406 & .3619271 \\
\hline Platte & .1514264 & .152455 & 0.99 & 0.323 & -.1518005 & .4546533 \\
\hline Schraube & -.1702035 & .4908041 & -0.35 & 0.730 & -1.146393 & .8059861 \\
\hline Sonstiges & -.1375367 & .3555997 & -0.39 & 0.700 & -.8448102 & .5697368 \\
\hline _cons & .2246301 & .1215175 & 1.85 & 0.068 & -.0170633 & .4663235 \\
\hline
\end{tabular}


Tabelle 23: Häufigkeiten der Frakturtypen und jeweilige Versorgungsart

\begin{tabular}{|c|c|c|c|c|c|}
\hline Frakturtyp & $\begin{array}{c}\text { Art der } \\
\text { Sonstiges }\end{array}$ & $\begin{array}{r}\text { Versorgung } \\
\text { gesch } \\
\text { geschloss }\end{array}$ & $\begin{array}{l}\text { initial of } \\
\text { lossen } \\
\text { konservat }\end{array}$ & $\begin{array}{l}\text { oder } \\
\text { offen }\end{array}$ & Total \\
\hline \multirow[t]{2}{*}{ Querfraktur } & 0 & 9 & 1 & 5 & \\
\hline & 0.00 & 60.00 & 6.67 & 33.33 & 100.00 \\
\hline \multirow[t]{2}{*}{ Schrägfraktur } & 0 & 10 & 2 & 3 & \\
\hline & 0.00 & 66.67 & 13.33 & 20.00 & 100.00 \\
\hline \multirow[t]{2}{*}{ Spiralfraktur } & 0 & 22 & 7 & 2 & \\
\hline & 0.00 & 70.97 & 22.58 & 6.45 & 100.00 \\
\hline \multirow[t]{2}{*}{ Trümmerfraktur } & 0 & 1 & 0 & 0 & 1 \\
\hline & 0.00 & 100.00 & 0.00 & 0.00 & 100.00 \\
\hline \multirow[t]{2}{*}{ nur Mehrfragmentfrakt } & 0 & 2 & 0 & 5 & 7 \\
\hline & 0.00 & 28.57 & 0.00 & 71.43 & 100.00 \\
\hline \multirow[t]{2}{*}{ nur Femurschaftfraktu } & 0 & 11 & 2 & 0 & 13 \\
\hline & 0.00 & 84.62 & 15.38 & 0.00 & 100.00 \\
\hline \multirow[t]{2}{*}{ nur Femurfraktur } & 1 & 7 & 7 & 0 & 15 \\
\hline & 6.67 & 46.67 & 46.67 & 0.00 & 100.00 \\
\hline \multirow[t]{2}{*}{ Total } & 1 & 62 & 19 & 15 & 97 \\
\hline & 1.03 & 63.92 & 19.59 & 15.46 & 100.00 \\
\hline
\end{tabular}


Tabelle 24: Komplikationswahrscheinlichkeit im jeweiligen Alter

Zeilen 1 - 4: Die logistische Regression zeigt einen signifikanten Unterschied des Komplikationsrisikos bei den unter Dreijährigen im Vergleich zu den drei- bis achteinhalbjährigen Patient(inn)en (Koeffizient $-0,28, P=0,015$ ) und den zwölf- bis dreizehnjährigen Patient(inn)en (Koeffizient 0,27, $P=0,066$ ). Jeweils unterschiedene Altersgruppen: Drei- bis Achteinhalbjährige, Achteinhalb- bis Zwölfjährige, Zwölf- bis Dreizehnjährige, über Vierzehnjährige. Zeilen 5 - 7: Kubische Regression Alter und Komplikationen. Das Risiko nimmt zunächst ab bis zu einem Alter von circa 4,5 Jahren. Danach steigt es bis zum einem Alter von circa zwölf Jahren wieder an und fällt im Anschluss erneut signifikant ab. Zeile 10: R-square zeigt, dass $20 \%$ der Variation bei Komplikationen allein durch die fünf Altersgruppen erklärt werden können (1).

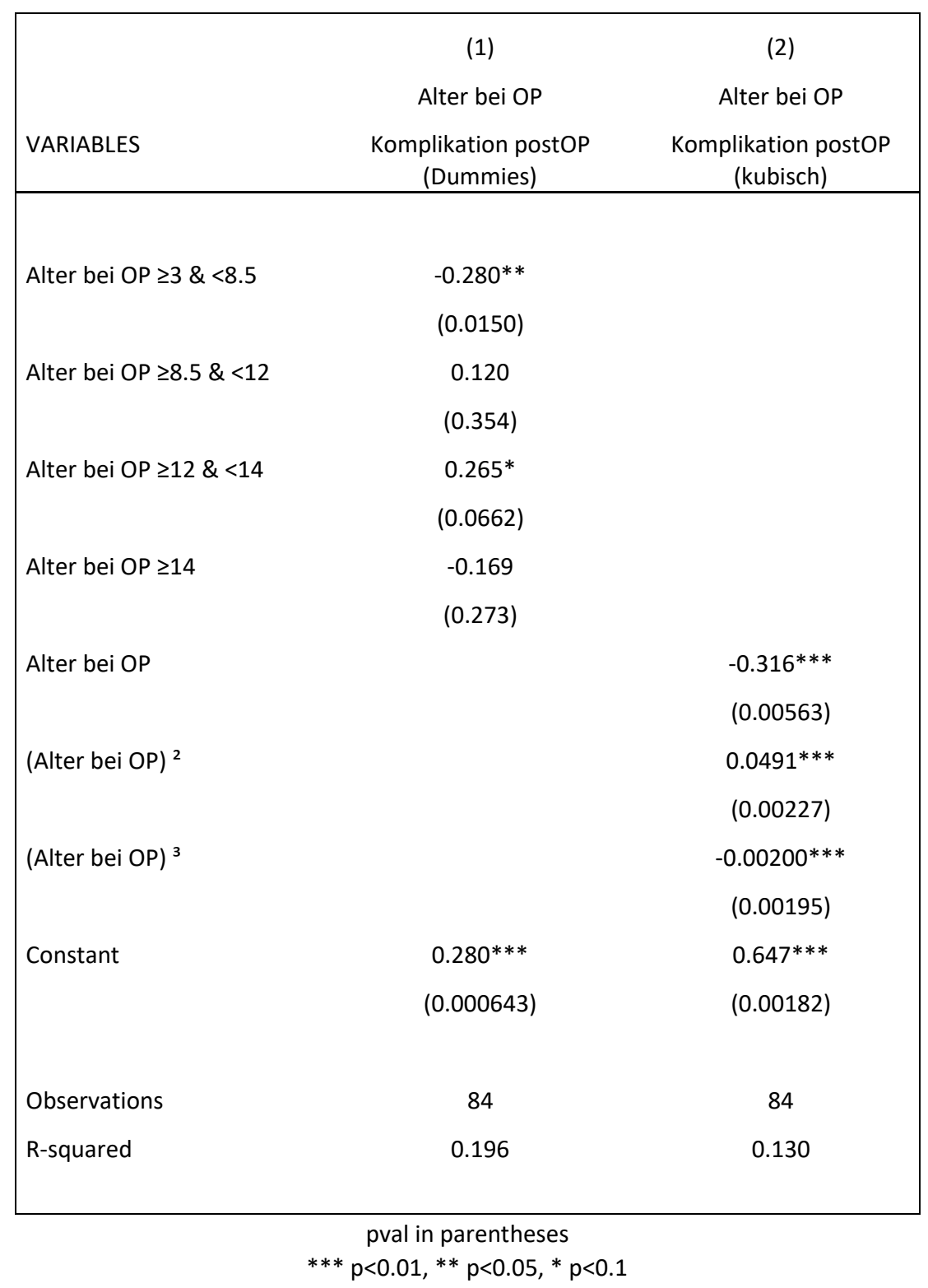


Tabelle 25: Multi-Variablen-Modell Alter, Drahtverhältnisse und Komplikationen

\begin{tabular}{|c|c|}
\hline VARIABLES & $\begin{array}{l}\text { Alter und Verhältnis Drähte } \\
\text { Komplikation post OP }\end{array}$ \\
\hline Alter bei OP $\geq 3 \&<8.5$ & $\begin{array}{l}-0.274 * \\
(0.0507)\end{array}$ \\
\hline Alter bei OP $\geq 8.5 \&<12$ & $\begin{array}{l}0.0767 \\
(0.627)\end{array}$ \\
\hline Alter bei $O P \geq 12 \&<14$ & $\begin{array}{c}0.424^{*} \\
(0.0560)\end{array}$ \\
\hline Alter bei $O P \geq 14$ & $\begin{array}{l}-0.235 \\
(0.412)\end{array}$ \\
\hline Verhältnis $\geq 1.50 \&<2$ & $\begin{array}{l}0.209 \\
(0.136)\end{array}$ \\
\hline Verhältnis $\geq 2$ & $\begin{array}{l}0.0268 \\
(0.845)\end{array}$ \\
\hline Constant & $\begin{array}{c}0.221^{*} \\
(0.0750)\end{array}$ \\
\hline Observations & 49 \\
\hline R-squared & 0.304 \\
\hline
\end{tabular}

Tabelle 26: Altersgruppen in 2-Jahres-Abständen im Vergleich und ihr jeweiliges Komplikationsrisiko

\begin{tabular}{r|crc} 
Source & SS & df & MS \\
\hline $\begin{array}{r}\text { Model } \\
\text { Residual }\end{array}$ & 2.58176268 & 7 & .36882324 \\
12.6563326 & 76 & .166530692 \\
\hline Total & 15.2380952 & 83 & .183591509
\end{tabular}

$$
\begin{array}{lrr}
\text { Number of obs } & = & 84 \\
\text { F 7, 76) } & =2.21 \\
\text { Prob }>\text { F } & =0.0421 \\
\text { R-squared } & =0.1694 \\
\text { Adj R-squared } & =0.0929 \\
\text { Root MSE } & =.40808
\end{array}
$$

\begin{tabular}{r|rrrrrr}
\hline Komplikati e & Coef. & Std. Err. & $t$ & P > t l & [95\% Conf. Interval] \\
\hline A2_4 & -.1461538 & .1518489 & -0.96 & 0.339 & -.4485872 & .1562795 \\
A4_6 & -.3 & .2235156 & -1.34 & 0.184 & -.7451698 & .1451698 \\
A6_8 & -.3 & .1935702 & -1.55 & 0.125 & -.6855284 & .0855284 \\
A8_10 & .1444444 & .1875006 & 0.77 & 0.443 & -.2289954 & .5178843 \\
A10_12 & .0333333 & .2107325 & 0.16 & 0.875 & -.3863768 & .4530435 \\
A12_14 & .2454545 & .1783037 & 1.38 & 0.173 & -.1096681 & .6005772 \\
A14_plus & -.1888889 & .1875006 & -1.01 & 0.317 & -.5623287 & .1845509 \\
_cons & .3 & .1290468 & 2.32 & 0.023 & .0429811 & .5570189
\end{tabular}


Tabelle 27: Frakturtypen bei Kindern unter drei Jahren (Spalte 1). Besonders viele, 44\% der unter Dreijährigen, litten unter einer Spiralfraktur. Sie stellten fast ein Drittel aller Patient(inn)en dar, die eine Spiralfraktur erlitten hatten $(n=32)$. Die zweithäufigste Fraktur der unter Dreijährigen war die Schrägfraktur: Fünf von ihnen zogen sich eine solche zu. Damit machten sie ein Drittel aller Patient(inn)en aus, die unter einer Schrägfraktur litten $(n=15)$. Frakturtyp 1 = Querfraktur, 2 = Schrägfraktur, $3=$ Spiralfraktur, $4=$ Trümmerfraktur, 5 = Mehrfragmentfraktur, 6 = „Femurschaftfraktur", 7 = nicht zuzuordnen.

\begin{tabular}{|c|c|c|c|}
\hline $\begin{array}{r}\text { Frakturtyp } \\
\text { (1=Querfra } \\
\text { ktur, } \\
2=\text { Schrägfr } \\
\text { aktur, } \\
\text { 3=Spiralfr } \\
\text { aktur, } \\
\text { 4=Trümmerf } \\
\text { raktur, } 5\end{array}$ & & 1 & Total \\
\hline 1 & $\begin{array}{r}12 \\
15.79\end{array}$ & $12.00^{3}$ & $\begin{array}{r}15 \\
14.85\end{array}$ \\
\hline 2 & $\begin{array}{r}10 \\
13.16\end{array}$ & $\begin{array}{r}5 \\
20.00\end{array}$ & $\begin{array}{r}15 \\
14.85\end{array}$ \\
\hline 3 & $\begin{array}{r}21 \\
27.63\end{array}$ & $\begin{array}{r}11 \\
44.00\end{array}$ & $\begin{array}{r}32 \\
31.68\end{array}$ \\
\hline 4 & $\begin{array}{r}0 \\
0.00\end{array}$ & $\begin{array}{r}1 \\
4.00\end{array}$ & $\begin{array}{r}1 \\
0.99\end{array}$ \\
\hline 5 & $\begin{array}{r}7 \\
9.21\end{array}$ & $\begin{array}{r}0 \\
0.00\end{array}$ & $\begin{array}{r}7 \\
6.93\end{array}$ \\
\hline 6 & $\begin{array}{r}12 \\
15.79\end{array}$ & $\begin{array}{r}4 \\
16.00\end{array}$ & $\begin{array}{r}16 \\
15.84\end{array}$ \\
\hline 7 & $\begin{array}{r}14 \\
18.42\end{array}$ & $\begin{array}{r}1 \\
4.00\end{array}$ & $\begin{array}{r}15 \\
14.85\end{array}$ \\
\hline Total & $\begin{array}{r}76 \\
100.00\end{array}$ & $\begin{array}{r}25 \\
100.00\end{array}$ & $\begin{array}{r}101 \\
100.00\end{array}$ \\
\hline
\end{tabular}


Tabelle 28: Frakturtypen der drei- bis achteinhalbjährigen Patient(inn)en. Diese Altersgruppe erlitt hauptsächlich Spiralfrakturen (38\%). Frakturtyp $1=$ Querfraktur, $2=$ Schrägfraktur, $3=$ Spiralfraktur, 4 = Trümmerfraktur, 5 = Mehrfragmentfraktur, $6={ }_{\text {„Femurschaftfraktur“ }}$, $7=$ nicht zuzuordnen.

\begin{tabular}{|c|c|c|}
\hline $\begin{array}{r}\text { Frakturtyp } \\
\text { (1=Querfra } \\
\text { ktur, } \\
2=\text { Schrägfr } \\
\text { aktur, } \\
\text { 3=Spiralfr } \\
\text { aktur, } \\
\text { 4=Trümmerf } \\
\text { raktur, } 5\end{array}$ & $\begin{array}{c}\text { Alter bei OP }>=3 \& \\
<8.5 \\
0\end{array}$ & Total \\
\hline 1 & $\begin{array}{r}4 \\
16.67\end{array}$ & $\begin{array}{r}14 \\
16.67\end{array}$ \\
\hline 2 & $\begin{array}{r}9 \\
15.00\end{array}$ & $\begin{array}{r}13 \\
15.48\end{array}$ \\
\hline 3 & $\begin{array}{r}19 \\
31.67\end{array}$ & $\begin{array}{r}28 \\
33.33\end{array}$ \\
\hline 4 & $\begin{array}{r}1 \\
1.67\end{array}$ & $\begin{array}{r}1 \\
1.19\end{array}$ \\
\hline 5 & $\begin{array}{r}5 \\
8.33\end{array}$ & $\begin{array}{r}7 \\
8.33\end{array}$ \\
\hline 6 & $\begin{array}{r}10 \\
16.67\end{array}$ & $\begin{array}{r}14 \\
16.67\end{array}$ \\
\hline 7 & $\begin{array}{r}6 \\
10.00\end{array}$ & $\begin{array}{r}7 \\
8.33\end{array}$ \\
\hline Total & $\begin{array}{r}60 \\
100.00\end{array}$ & $\begin{array}{r}84 \\
100.00\end{array}$ \\
\hline
\end{tabular}


Tabelle 29: Frakturtypen der über achteinhalbjährigen Patient(inn)en (1). Es traten am häufigsten Spiralfrakturen auf (23\%). Frakturtyp $1=$ Querfraktur, $2=$ Schrägfraktur, $3=$ Spiralfraktur, 4 $=$ Trümmerfraktur, 5 = Mehrfragmentfraktur, $6=$ „Femurschaftfraktur", 7 = nicht zuzuordnen

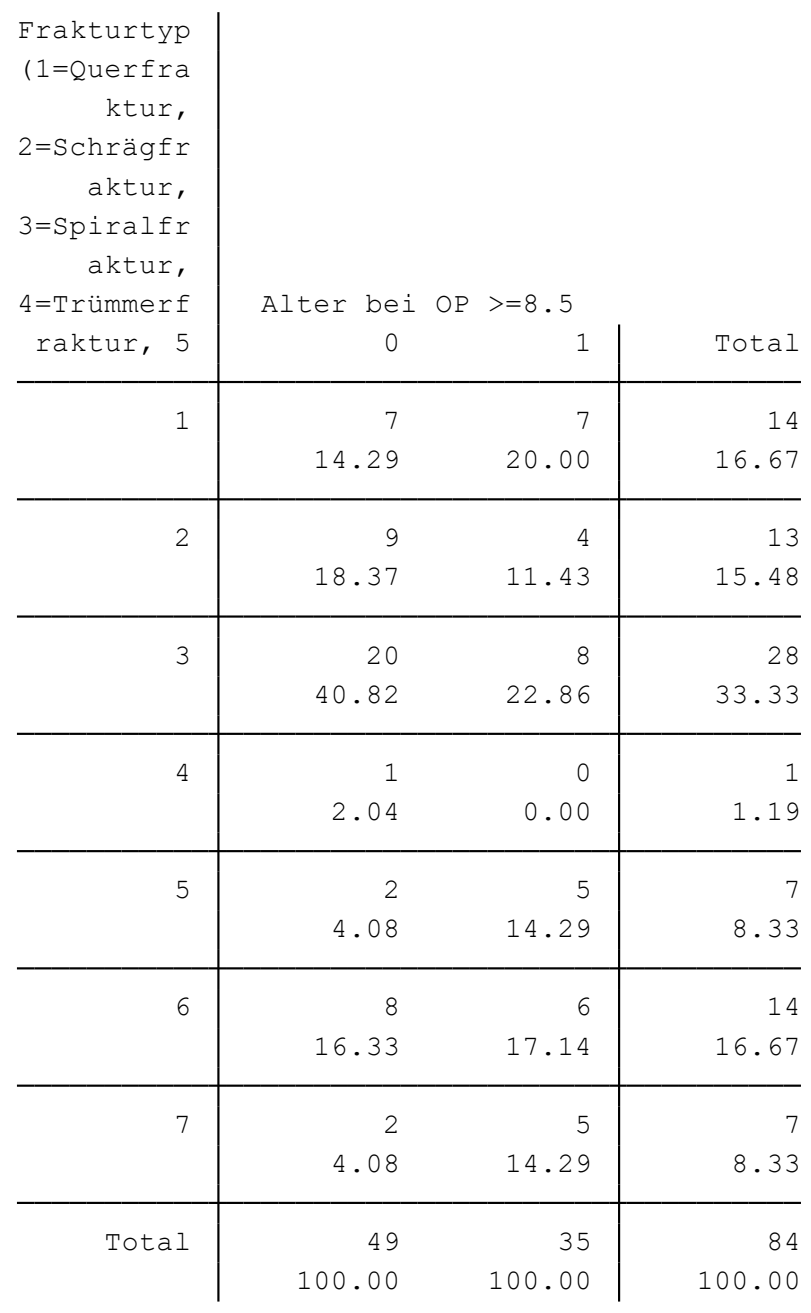

Tabelle 30: Multi-Variablen-Modell Body-Mass-Index (BMI), Alter und postoperative Komplikationen

\begin{tabular}{|c|c|c|c|}
\hline VARIABLES & $\begin{array}{c}\text { (1) } \\
\text { BMI } \\
\text { Komplikation postOP }\end{array}$ & $\begin{array}{c}\text { (2) } \\
\text { Alter on BMI } \\
\text { BMI } \\
\end{array}$ & $\begin{array}{c}\text { (3) } \\
\text { Alter und BMI } \\
\text { Komplikation postOP }\end{array}$ \\
\hline Alter bei OP & & & $-0.225 *$ \\
\hline & & & $(0.0773)$ \\
\hline$(\text { Alter bei OP })^{2}$ & & & $\begin{array}{c}0.0368 * * \\
(0.0386)\end{array}$ \\
\hline$(\text { Alter bei OP) })^{3}$ & & & $\begin{array}{c}-0.00153^{* *} \\
(0.0327)\end{array}$ \\
\hline BMI & $\begin{array}{c}0.000231 \\
(0.983)\end{array}$ & & $\begin{array}{c}-0.00293 \\
(0.796)\end{array}$ \\
\hline Alter bei OP absolut & & $\begin{array}{l}0.351 * * * \\
(0.00404)\end{array}$ & \\
\hline Constant & $\begin{array}{c}0.223 \\
(0.260)\end{array}$ & $\begin{array}{c}15.26^{* * *} \\
(0)\end{array}$ & $\begin{array}{c}0.532^{*} \\
(0.0709)\end{array}$ \\
\hline Observations & 66 & 65 & 65 \\
\hline R-squared & 0.000 & 0.124 & 0.101 \\
\hline
\end{tabular}


Tabelle 31: logistische Regression Komplikationen und Perzentilkurven (kein signifikantes Ergebnis). Einteilung der Perzentilkurven BMI für Alter ( $1=$ starkes Untergewicht $<\mathrm{P} 3,2=$ Untergewicht P3 - P9,9, 3 = Normalgewicht P10 - P89,9, $4=$ Übergewicht P90 - P97, $5=$ starkes Übergewicht $>$ P97).

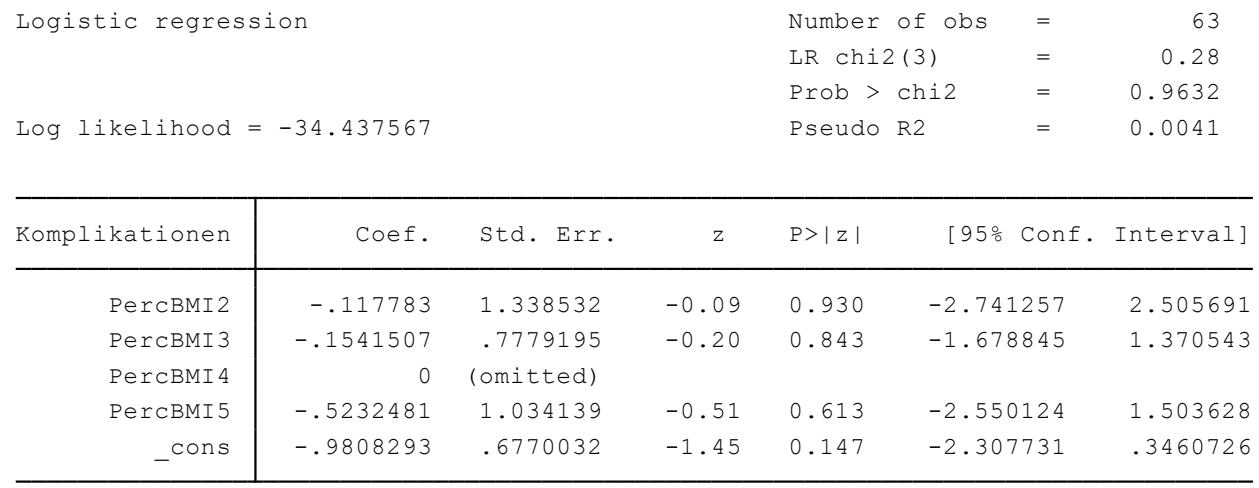

- tab BMIfrAlterlstarkesUnterg, gen (PercBMI)

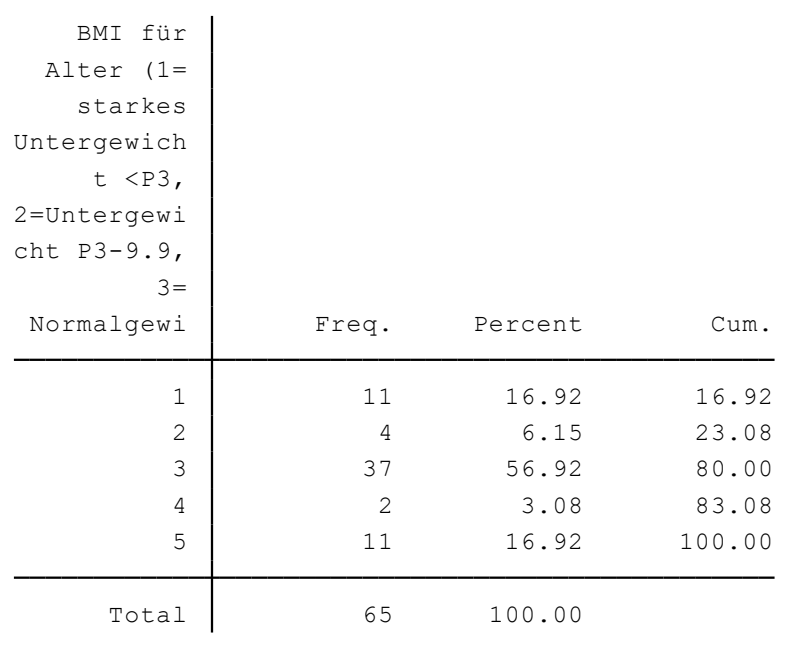

Tabelle 32: t-Test Wahrscheinlichkeit von Komplikationen bei konservativer Therapie. $\mathrm{P}=0.04$ zeigt, dass konservative Therapien signifikant weniger Komplikationen aufweisen (um 7\%) gegenüber den anderen Behandlungsmöglichkeiten (konservative Therapie mittleres Komplikationsrisiko $16 \%$, andere Behandlungsmethoden $23 \%$ ).

One-sample $t$ test

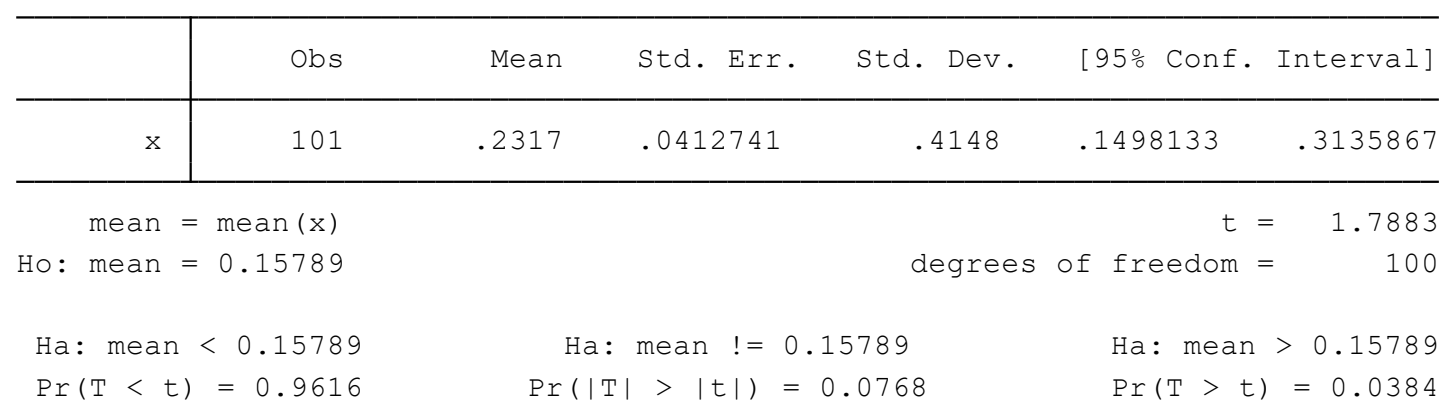


Tabelle 33: t-Test Wahrscheinlichkeit von Komplikationen bei offener Operation (33\%) gegenüber allen anderen Behandlungsmethoden (20\%)

One-sample $t$ test

\begin{tabular}{|c|c|c|c|c|c|c|}
\hline & Obs & Mean & Std. Err. & Std. Dev. & [95\% Conf. & Interval] \\
\hline $\mathrm{x}$ & 101 & .197 & .0412741 & .4148 & .1151133 & .2788867 \\
\hline mean $=$ & $\operatorname{mean}(\mathrm{x})$ & & & & $t=$ & -3.3023 \\
\hline Ho: $\operatorname{mean}=$ & 0.3333 & & & degrees & freedom $=$ & 100 \\
\hline Ha: mean & $<0.3333$ & & mean $!=0$ & 333 & Ha: mear & $n>0.3333$ \\
\hline $\operatorname{Pr}(T<t)$ & $=0.0007$ & $\operatorname{Pr}($ & $|>| t \mid)=$ & .0013 & $\operatorname{Pr}(T>t)$ & )$=0.9993$ \\
\hline
\end{tabular}

Tabelle 34: t-Test Wahrscheinlichkeit von Komplikationen bei offener Operation (33\%) gegenüber geschlossener Operation (21\%)

One-sample $t$ test

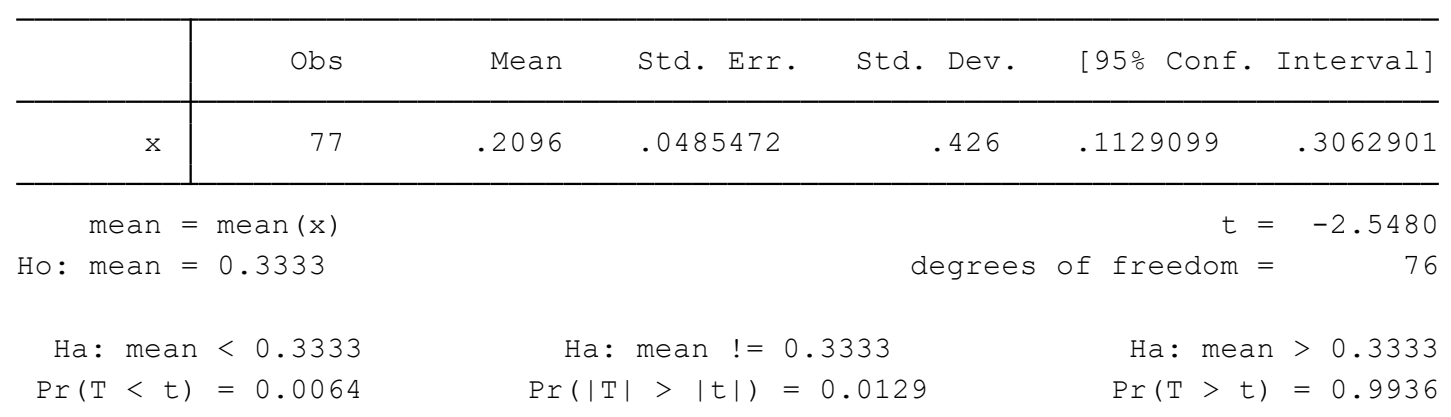


Tabelle 35: Regression von Komplikationswahrscheinlichkeit, Frakturtyp und Art der Operation. In Klammern ist der dazugehörige P-Wert angegeben. Die Komplikationswahrscheinlichkeit ergibt sich aus a) dem Frakturtyp (Zeile 1-7) plus b) der Art der Operation (Zeile 8 oder 24) plus c) dem Interaktionsterm von Operationsart und Frakturtyp (Zeile 9-23). Dies alles gilt im Vergleich zum Frakturtyp 1 (= Querfraktur) und der konservativen Therapie.

\begin{tabular}{|c|c|}
\hline VARIABLES & $\begin{array}{c}\text { Frakturtyp und Art der Operation } \\
\text { Komplikation post OP }\end{array}$ \\
\hline Frakturtyp $=2$, Schrägfraktur & $\begin{array}{l}0.0694 \\
(0.700)\end{array}$ \\
\hline Frakturtyp $=3$, Spiralfraktur & $\begin{array}{c}0.173 \\
(0.263)\end{array}$ \\
\hline Frakturtyp $=4$, Trümmerfraktur & $\begin{array}{l}-0.104 \\
(0.813)\end{array}$ \\
\hline Frakturtyp $=5$, nur Mehrfragmentfraktur & $\begin{array}{l}-0.104 \\
(0.749)\end{array}$ \\
\hline Frakturtyp $=6$, nur Femurschaftfraktur & $\begin{array}{c}0.221 \\
(0.198)\end{array}$ \\
\hline Frakturtyp $=7$, nicht zuzuordnen & $\begin{array}{l}-0.0122 \\
(0.946)\end{array}$ \\
\hline Operation_offen = 1 & $\begin{array}{l}0.537^{* *} \\
(0.0336)\end{array}$ \\
\hline 1b.Frakturtyp\#0b.Operation_offen & 0 \\
\hline 1b.Frakturtyp\#10.Operation_offen & 0 \\
\hline 2o.Frakturtyp\#0b.Operation_offen & 0 \\
\hline 2.Frakturtyp\#1.Operation_offen & $\begin{array}{l}-0.336 \\
(0.346)\end{array}$ \\
\hline 3o.Frakturtyp\#0b.Operation_offen & 0 \\
\hline 3.Frakturtyp\#1.Operation_offen & $\begin{array}{l}-0.773 * * \\
(0.0464)\end{array}$ \\
\hline 4o.Frakturtyp\#0b.Operation_offen & 0 \\
\hline 4o.Frakturtyp\#10.Operation_offen & 0 \\
\hline 5o.Frakturtyp\#0b.Operation_offen & 0 \\
\hline 5.Frakturtyp\#1.Operation_offen & $\begin{array}{l}-0.296 \\
(0.482)\end{array}$ \\
\hline 6o.Frakturtyp\#0b.Operation_offen & 0 \\
\hline 6o.Frakturtyp\#10.Operation_offen & 0 \\
\hline 7o.Frakturtyp\#0b.Operation_offen & 0 \\
\hline 7o.Frakturtyp\#10.Operation_offen & 0 \\
\hline Operation_geschlossen & $\begin{array}{l}0.0409 \\
(0.703)\end{array}$ \\
\hline Operation_sonst & $\begin{array}{l}-0.0510 \\
(0.907)\end{array}$ \\
\hline Constant & $\begin{array}{l}0.0632 \\
(0.701)\end{array}$ \\
\hline Observations & 101 \\
\hline R-squared & 0.103 \\
\hline
\end{tabular}


Tabelle 36: Regression von Komplikationen, Frakturtyp und Implantationsmaterial. Es konnten keine Kombinationen von Frakturtyp und Implantationsmaterial festgestellt werden, die das Komplikationsrisiko signifikant beeinflusst hätten. Querfraktur und ESINs wurden jeweils als Vergleichsgruppe eingesetzt. Beispielsweise war das Einsetzen einer Platte mit einem 15\% höheren Komplikationsrisiko gegenüber ESINs behaftet, jedoch ist das Ergebnis nicht signifikant (Koeffizient $0,15, t=0,99$ ). Auch bei den Frakturtypen gab es keine signifikanten Ergebnisse. Allein die „Femurschaftfraktur" (Frakturtyp 6) war vergleichbar ähnlich risikobehaftet wie die Querfraktur (Koeffizient 0,1, $t=0,67$, also nicht signifikant).

\begin{tabular}{r|ccc} 
Source & SS & df & MS \\
\hline $\begin{array}{r}\text { Model } \\
\text { Residual }\end{array}$ & 1.04963258 & 14 & .074973756 \\
16.0115919 & 83 & .192910746 \\
\hline Total & 17.0612245 & 97 & .175888912
\end{tabular}

$\begin{array}{lrr}\text { Number of obs } & = & 98 \\ \text { F }(14, \quad 83) & = & 0.39 \\ \text { Prob }>\text { F } & =0.9747 \\ \text { R-squared } & =0.0615 \\ \text { Adj R-squared } & = & -0.0968 \\ \text { Root MSE } & =.43922\end{array}$

\begin{tabular}{r|rrrrrr}
\hline Komplikation e & Coef. & Std. Err. & $t$ & P $|t|$ & [95\% Conf. & Interval] \\
\hline Frakturtyple h & & & & & & \\
2 & -.0378697 & .1628967 & -0.23 & 0.817 & -.3618647 & .2861252 \\
3 & .0159906 & .1411984 & 0.11 & 0.910 & -.2648474 & .2968286 \\
4 & -.07076 & .5676609 & -0.12 & 0.901 & -1.199815 & 1.058295 \\
5 & -.0544266 & .2385811 & -0.23 & 0.820 & -.5289549 & .4201017 \\
6 & .111219 & .1665988 & 0.67 & 0.506 & -.2201394 & .4425773 \\
7 & -.0870934 & .2093205 & -0.42 & 0.678 & -.5034236 & .3292367 \\
& & & & & & \\
ArtderVersor g & & & & & & \\
Fixateur ex. & -.1538701 & .343434 & -0.45 & 0.655 & -.8369465 & .5292064 \\
Gips & -.0377362 & .2092627 & -0.18 & 0.857 & -.4539512 & .3784789 \\
K-Draht & -.1539446 & .2828322 & -0.54 & 0.588 & -.7164864 & .4085973 \\
Marknagel & -.1702035 & .4908041 & -0.35 & 0.730 & -1.146393 & .8059861 \\
Overheadext n & .0248432 & .1694775 & 0.15 & 0.884 & -.3122406 & .3619271 \\
Platte & .1514264 & .152455 & 0.99 & 0.323 & -.1518005 & .4546533 \\
Schraube & -.1702035 & .4908041 & -0.35 & 0.730 & -1.146393 & .8059861 \\
Sonstiges & -.1375367 & .3555997 & -0.39 & 0.700 & -.8448102 & .5697368 \\
& & & & & & \\
_cons & .2246301 & .1215175 & 1.85 & 0.068 & -.0170633 & .4663235
\end{tabular}


Tabelle 37: Logistische Regression von Verhältnis Markraumdurchmesser zu ESIN-Drahtdicke und das Risiko für Komplikationen. Für das Verhältnis $\geq 1.5$ bis 2, besteht eine $20 \%$ ig höheres Komplikationsrisiko, als für den Fall Verhältnis $<1.5$. Für Verhältnis $\geq 2$ besteht kein signifikanter Zusammenhang. In Klammern sind die P-Werte angegeben.

\begin{tabular}{|c|c|c|}
\hline VARIABLES & $\begin{array}{l}\text { (1) } \\
\text { Verhältnis beider Drähte } \\
\text { Komplikation post OP }\end{array}$ & $\begin{array}{l}\text { (2) } \\
\text { Verhältnis beider Drähte } \\
\text { Komplikation post OP }\end{array}$ \\
\hline Verhältnis $\geq 1.5 \&<2$ & $\begin{array}{c}0.300 * \\
(0.0949)\end{array}$ & \\
\hline Verhältnis $\geq 2$ & $\begin{array}{l}0.0974 \\
(0.562)\end{array}$ & \\
\hline Verhältnis beider Drähte & & $\begin{array}{l}2.573 * * * \\
(0.00567)\end{array}$ \\
\hline 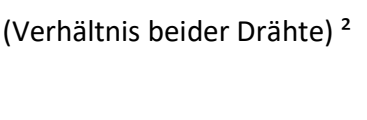 & & $\begin{array}{l}-0.741^{* * *} \\
(0.00651)\end{array}$ \\
\hline Observations & 48 & 46 \\
\hline Pseudo-R squared & 0,063 & 0,13 \\
\hline
\end{tabular}


Tabelle 38: Häufigkeiten von Komplikationen bei den jeweiligen Unfallgeschehen (1) im Vergleich zur Gruppe ohne Komplikationen (0).

\begin{tabular}{|c|c|c|c|}
\hline $\begin{array}{r}\text { Klassifizierung } \\
\text { Unfallhergang }(1= \\
\text { Sturz <1m, } 2=\text { Sturz }> \\
1 \mathrm{~m}, 3=\text { Sturz }>3 \mathrm{~m}, \\
4=\mathrm{Fah}\end{array}$ & $\begin{array}{r}\text { Komplikati } \\
0\end{array}$ & $\begin{array}{r}\text { post } \\
1\end{array}$ & Total \\
\hline Sturz $<=1 \mathrm{~m}$ & $\begin{array}{r}7 \\
8.97\end{array}$ & $\begin{array}{r}4 \\
19.05\end{array}$ & $\begin{array}{r}11 \\
11.11\end{array}$ \\
\hline Sturz $>1 \mathrm{~m}$ & $\begin{array}{r}4 \\
5.13\end{array}$ & $\begin{array}{r}3 \\
14.29\end{array}$ & $\begin{array}{r}7 \\
7.07\end{array}$ \\
\hline Sturz $>3 m$ & $\begin{array}{r}4 \\
5.13\end{array}$ & $\begin{array}{r}0 \\
0.00\end{array}$ & $\begin{array}{r}4 \\
4.04\end{array}$ \\
\hline Fahrrad & $\begin{array}{r}6 \\
7.69\end{array}$ & $\begin{array}{r}3 \\
14.29\end{array}$ & $\begin{array}{r}9 \\
9.09\end{array}$ \\
\hline Fußball & $\begin{array}{r}6 \\
7.69\end{array}$ & $\begin{array}{r}0 \\
0.00\end{array}$ & $\begin{array}{r}6 \\
6.06\end{array}$ \\
\hline Pferdesport & $\begin{array}{r}1 \\
1.28\end{array}$ & $\begin{array}{r}1 \\
4.76\end{array}$ & $\begin{array}{r}2 \\
2.02\end{array}$ \\
\hline sonstiger Sport & $\begin{array}{r}3 \\
3.85\end{array}$ & $\begin{array}{r}1 \\
4.76\end{array}$ & $\begin{array}{r}4 \\
4.04\end{array}$ \\
\hline Toben/Spielen & $\begin{array}{r}8 \\
10.26\end{array}$ & $\begin{array}{r}2 \\
9.52\end{array}$ & $\begin{array}{r}10 \\
10.10\end{array}$ \\
\hline Pkw-/Businsasse & $\begin{array}{r}8 \\
10.26\end{array}$ & $\begin{array}{r}0 \\
0.00\end{array}$ & $\begin{array}{r}8 \\
8.08\end{array}$ \\
\hline Fußgänger(in)_Pkw & $\begin{array}{r}4 \\
5.13\end{array}$ & $\begin{array}{r}2 \\
9.52\end{array}$ & $\begin{array}{r}6 \\
6.06\end{array}$ \\
\hline Fahrradfahrer(in)_Pkw & $\begin{array}{r}1 \\
1.28\end{array}$ & $\begin{array}{r}1 \\
4.76\end{array}$ & $\begin{array}{r}2 \\
2.02\end{array}$ \\
\hline Gewalt & $\begin{array}{r}1 \\
1.28\end{array}$ & $\begin{array}{r}0 \\
0.00\end{array}$ & $\begin{array}{r}1 \\
1.01\end{array}$ \\
\hline 'umgeknickt' & $\begin{array}{r}10 \\
12.82\end{array}$ & $\begin{array}{r}0 \\
0.00\end{array}$ & $\begin{array}{r}10 \\
10.10\end{array}$ \\
\hline Anpralltrauma & $\begin{array}{r}1 \\
1.28\end{array}$ & $\begin{array}{r}1 \\
4.76\end{array}$ & $\begin{array}{r}2 \\
2.02\end{array}$ \\
\hline Quetschtrauma & $\begin{array}{r}7 \\
8.97\end{array}$ & $\begin{array}{r}0 \\
0.00\end{array}$ & $\begin{array}{r}7 \\
7.07\end{array}$ \\
\hline kein Trauma & $\begin{array}{r}4 \\
5.13\end{array}$ & $\begin{array}{r}1 \\
4.76\end{array}$ & $\begin{array}{r}5 \\
5.05\end{array}$ \\
\hline Sonstiges & $\begin{array}{r}3 \\
3.85\end{array}$ & $\begin{array}{r}2 \\
9.52\end{array}$ & $\begin{array}{r}5 \\
5.05\end{array}$ \\
\hline Total & $\begin{array}{r}78 \\
100.00\end{array}$ & $\begin{array}{r}21 \\
100.00\end{array}$ & $\begin{array}{r}99 \\
100.00\end{array}$ \\
\hline
\end{tabular}


Tabelle 39: Multivariablen-Regression von Alter, Unfallmechanismus und Komplikationen. Demnach erleiden Kinder im Alter zwischen drei und achteinhalb Jahren mit einer Wahrscheinlichkeit von 31\% weniger Komplikationen als die unter Dreijährigen (Koeffizient -0.31). Alle Unfallursachen beziehen sich auf die Vergleichsgröße Kategorie "Sturz $\leq 1 \mathrm{~m}$ ". Im Rahmen eines Anpralltraumas zugezogene Frakturen (Koeffizient 0.5) oder als Fahrradfahrer(in) erlittene Brüche (Koeffizient 0.2), hatten zum Beispiel ein höheres Risiko Komplikationen zu erleiden (jedoch sind diese Ergebnisse nicht signifikant).

\begin{tabular}{r|ccc} 
Source & SS & df & MS \\
\hline $\begin{array}{r}\text { Model } \\
\text { Residual }\end{array}$ & 5.28306036 & 20 & .264153018 \\
9.31450062 & 61 & .152696731 \\
\hline Total & 14.597561 & 81 & .180216802
\end{tabular}

$\begin{array}{lrr}\text { Number of obs } & = & 82 \\ \mathrm{~F}(20, \quad 61) & = & 1.73 \\ \text { Prob }>\text { F } & =0.0527 \\ \text { R-squared } & =0.3619 \\ \text { Adj R-squared } & =0.1527 \\ \text { Root MSE } & =.39076\end{array}$

\begin{tabular}{|c|c|c|c|c|c|c|}
\hline Komplikationen & Coef. & std. Err. & $t$ & $P>|t|$ & [95\% Conf. & Interval] \\
\hline \multicolumn{7}{|l|}{ Unfallhergang } \\
\hline Sturz $>1 \mathrm{~m}$ & .1317847 & .2369028 & 0.56 & 0.580 & -.3419317 & .605501 \\
\hline sturz $>3 m$ & -.3600234 & .2398315 & -1.50 & 0.138 & -.8395961 & .1195493 \\
\hline Fahrrad & .0904366 & .1929503 & 0.47 & 0.641 & -.2953914 & .4762646 \\
\hline Fußball & -.2571206 & .218787 & -1.18 & 0.244 & -.6946123 & .1803711 \\
\hline Pferdesport & .0728668 & .3243203 & 0.22 & 0.823 & -.5756516 & .7213853 \\
\hline sonstiger S.. & -.1602591 & .2601744 & -0.62 & 0.540 & -.6805099 & .3599917 \\
\hline Toben/Spielen & -.3046109 & .1823748 & -1.67 & 0.100 & -.6692918 & .0600701 \\
\hline Pkw-/Busins e & -.2618037 & .2189728 & -1.20 & 0.236 & -.6996669 & .1760595 \\
\hline Fußgänger $(i \sim w$ & -.0471737 & .2247288 & -0.21 & 0.834 & -.4965466 & .4021992 \\
\hline Fahrradfahr w & .1518265 & .3131673 & 0.48 & 0.630 & -.4743902 & .7780432 \\
\hline Gewalt & -.4491894 & .4159441 & -1.08 & 0.284 & -1.280921 & .3825423 \\
\hline 'umgeknickt' & -.2872413 & .2077753 & -1.38 & 0.172 & -.7027137 & .1282311 \\
\hline Anpralltrauma & .5394659 & .4200444 & 1.28 & 0.204 & -.3004648 & 1.379397 \\
\hline Quetschtrauma & -.2578041 & .2022381 & -1.27 & 0.207 & -.6622042 & .146596 \\
\hline kein Trauma & .1000747 & .2639227 & 0.38 & 0.706 & -.4276713 & .6278208 \\
\hline Sonstiges & -.1002041 & .2189907 & -0.46 & 0.649 & -.538103 & .3376948 \\
\hline Alter_OP_3_8 J & -.3238962 & .1307344 & -2.48 & 0.016 & -.5853158 & -.0624766 \\
\hline Alter_OP_ $8 \overline{\mathrm{k}} 5 \sim \mathrm{J}$ & .0113447 & .1497962 & 0.08 & 0.940 & -.2881913 & .3108806 \\
\hline Alter_OP_1 3_J & .1218645 & .1730737 & 0.70 & 0.484 & -.2242177 & .4679467 \\
\hline Alter_OP_ü13_J & -.1659769 & .1947601 & -0.85 & 0.397 & -.5554238 & .22347 \\
\hline cons & .4491894 & .142523 & 3.15 & 0.003 & .164197 & .7341817 \\
\hline
\end{tabular}

Tabelle 40: Quadratische Regression von Komplikationsrate und Dauer zwischen Metallimplantation und -entfernung

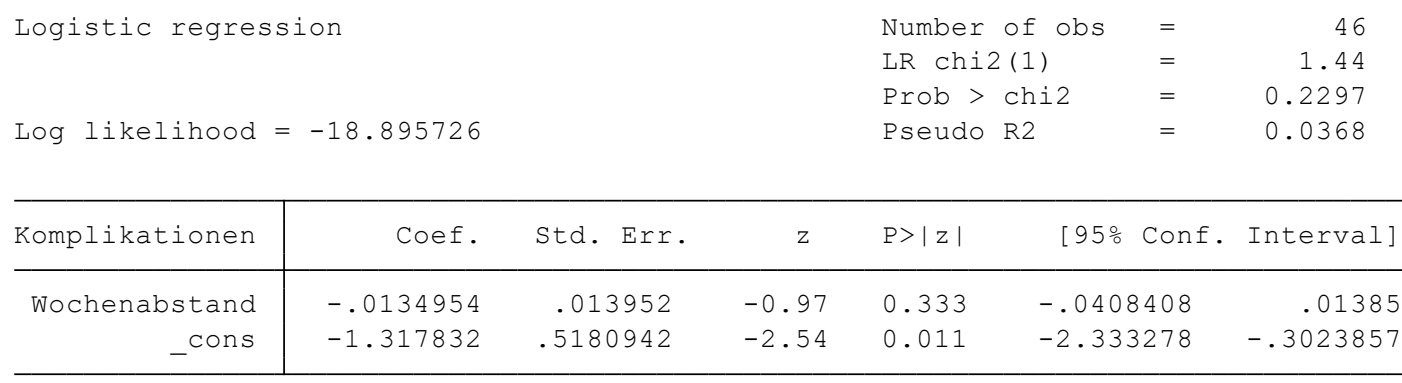


Tabelle 41: Quadratische Regression des Komplikationsrisikos und der Liegedauer mit signifikanten Ergebnissen. Es liegt jedoch ein Kausalitätsproblem vor.

\begin{tabular}{|lc|}
\hline & $\begin{array}{c}\text { Frakturtyp und Art der Operation } \\
\text { Komplikation post OP }\end{array}$ \\
\hline VARIABLES & $-0.0383^{*}$ \\
Liegedauer Anzahl Tage & $(0.0947)$ \\
Liegedauer ${ }^{2}$ & $0.00122^{*}$ \\
& $(0.0701)$ \\
Observations & 92 \\
& pval in parentheses \\
& $* * * p<0.01, * * p<0.05, * p<0.1$
\end{tabular}

Tabelle 42: Signifikanztest (t-Test) bestätigt, dass pathologische Frakturen das Komplikationsrisiko um 9\% erhöhen (P-Wert $=0,015)$. Die Komplikationswahrscheinlichkeit für Patienten mit pathologischen Frakturen (Ho mean) beträgt 30\%, das Risiko für Komplikationen aller Patienten (x mean) liegt bei $21 \%$.

One-sample $t$ test

\begin{tabular}{|c|c|c|c|c|c|c|c|}
\hline & Obs & Mean & Std. Err. & Std. & Dev. & [95\% Conf. & Interval] \\
\hline$x$ & 101 & .2096 & .0411945 & & .414 & .1278712 & .2913288 \\
\hline \multicolumn{2}{|c|}{$\operatorname{mean}=\operatorname{mean}(\mathrm{x})$} & & & & \multirow[b]{2}{*}{ degrees } & $t=$ & -2.1945 \\
\hline Ho: mean & $=0.30$ & & & & & of freedom & 100 \\
\hline $\mathrm{Ha}: \mathrm{m} \epsilon$ & $a n<0.30$ & & mean $!=c$ & 30 & & $\mathrm{Ha}: \mathrm{me}$ & $a n>0.30$ \\
\hline $\operatorname{Pr}(\mathrm{T}<t$ & )$=0.0153$ & $\operatorname{Pr}$ & $|>| t \mid)=$ & .0305 & & $\operatorname{Pr}(\mathrm{T}>\mathrm{t}$ & )$=0.9847$ \\
\hline
\end{tabular}




\section{Quellen}

\subsection{Literaturverzeichnis}

Bandyopadhyay R, Mekherjee A (2013): Short Term Complications of Titanium Elastic Nail in the Treatment of Diaphyseal Fracture of the Femur in Children. Open Orthop J, 7, $12-17$

Biberthaler P, Oestern H-J: Traumatologie. In: Henne-Bruns D (Hrsg.): Chirurgie (Duale Reihe). 4. Auflage; Thieme, Stuttgart 2012, 651-811

Böhm R: Oberschenkel. In: von Laer L (Hrsg.): Das verletzte Kind. Thieme, Stuttgart 2007, 75-81

Böhmann J, Ellsäßer G (2004): Bevölkerungsbezogenes Unfallmonitoring von Kinderunfällen in einer deutschen Stadt. Monatss Kinderheilkd 152, 299-306 Bonjour J-P, Chevalley T (2014): Pubertal timing, bone acquisition, and risk of fracture throughout life. Endocr Rev $\underline{35}$, 820-847

Bonjour J-P, Theintz G, Law F, Slosman D, Rizzoli R (1994): Peak bone mass. Osteoporosis Int $\underline{4}, 7-13$

Buechsenschuetz KE, Mehlman CT, Shaw KJ, Crawford AH, Immerman EB (2002): Femoral shaft fractures in children: traction and casting versus elastic stable intramedullary nailing. J Trauma 53, 914-921

Bühren V: Femurschaft. In: Rüter A, Trentz O, Wagner M (Hrsg.): Unfallchirurgie. 2. Auflage; Urban und Fischer/Elsevier, München 2004, 997-1013

Bühren V, Keel MJ, Marzi I: Verletzungen bei Kindern. In: Bühren V, Keel MJ, Marzi I (Hrsg.): Checkliste Traumatologie (Checklisten der aktuellen Medizin). 8. Auflage; Thieme, Stuttgar 2016, 266-284

Chevalley T, Bonjour J-P, van Rietbergen B, Ferrari S, Rizzoli R (2011): Fractures during childhood and adolescence in healthy boys: relation with bone mass, microstructure, and strength. J Clin Endocrinol Metab $\underline{96}$, 3134-3142

Chevalley T, Bonjour J-P, van Rietbergen B, Rizzoli R, Ferrari S (2012): Fractures in healthy females followed from childhood to early adulthood are associated with later menarcheal age and with impaired bone microstructure at peak bone mass. J Clinical Endocrinology Metab 97, 4174-4181 
Cooper DML, Ahamed Y, Macdonald HM, McKay HA (2008): Characterising cortical density in the mid-tibia: intra-individual variation in adolescent girls and boys. $\mathrm{Br} J$ Sports Med $\underline{42}, 690-695$

Dietz HG: Grundlagen der konservativen Frakturbehandlung. In: Dietz HG, Illing P, Schmittenbecher PP, Slongo T, Sommerfeldt DW: Praxis der Kinder- und Jugendtraumatologie. Springer, Heidelberg 2011, 55-65

Dietz HG, Schlickewei W (2011): Femurschaftfrakturen im Kindesalter. Unfallchirurg $\underline{114}, 382-387$

Dietz HG, Schmittenbecher PP, Illing P: Intramedulläre Osteosynthese im Wachstumsalter. Urban und Schwarzenberg/Elsevier, München 1997 Ellsäßer G (2006): Epidemiologische Analyse von Unfällen bei Kindern unter 15 Jahren in Deutschland - Ausgangspunkt für die Unfallprävention. Gesundheitswesen $\underline{68}$, 421428

Ferrari SL, Chevalley T, Bonjour J-P, Rizzoli R (2006): Childhood fractures are associated with decreased bone mass gain during puberty: an early marker of persistent bone fragility? J Bone Miner Res 21, 501-507

Flinck M, von Heideken Johan, Janarv P-M, Watz V, Riad J (2015): Biomechanical comparison of semi-rigid pediatric locking nail versus titanium elastic nails in a femur fracture model. J Child Orthop $\underline{9}, 77-84$

Fournier P-E, Rizzoli R, Slosman D-O, Theintz G, Bonjour J-P (1997): Asynchrony between the rates of standing height gain and bone mass accumulation during puberty. Osteoporosis Int $\underline{7}, 525-532$

Gresing T: Hüftgelenk und Oberschenkel. In: Dietz HG, Illing P, Schmittenbecher PP, Slongo T, Sommerfeld DW (Hrsg.): Praxis der Kinder- und Jugendtraumatologie.

Springer, Heidelberg 2011

Hedström EM, Svensson O, Bergström U, Michno P (2010): Epidemiology of fractures in children and adolescents. Acta Orthop $\underline{81}, 148-153$

Heimkes B, Günther C: Erworbene Wachstumsstörungen. In: Reinhardt P, Nicolai T, Zimmer K-P (Hrsg.): Therapie der Krankheiten im Kindes- und Jugendalter. 9. Auflage; Springer, Berlin 2014, 371-371

Jauquier N, Doerfler M, Haecker F-M, Hasler C, Zambelli P-Y, Lutz N (2010): Immediate hip spica is as effective as, but more efficient than, flexible intramedullary nailing for femoral shaft fractures in pre-school children. J Child Orthop 4 , 461-465 
Jones IE, Williams SM, Dow N, Goulding A (2002): How many children remain fracturefree during growth? A longitudinal study of children and adolescents participating in the Dunedin Multidisciplinary Health and Developement Study. Osteoporosis Int, 990995

Jubel A, Andermahr J, Prokop A, Bergmann H, Isenberg J, Rehm KE (2004): Pitfalls and complications of elastic stable intramedullary nailing (ESIN) of femoral fractures in infancy. Unfallchirurg 107, 744-749

Kahl H, Dortschy R, Ellsäßer G (2007): Verletzungen bei Kindern und Jugendlichen (1-17 Jahren) und Umsetzungen von persönlichen Schutzmaßnahmen: Ergebnisse des bundesweiten Kinder- und Jugendgesundheitssurveys (KiGGS). Bundesgesundheitsbl Gesundheitsforsch Gesundheitsschutz $\underline{50}, 718-727$

Kaiser MM, Zachert G, Wendlandt R, Rapp M, Eggert R, Stratmann C, Wessel LM, Schulz AP, Kienast BJ (2011): Biomechanical analysis of a synthetic femoral spiral fracture model: Do end caps improve retrograde flexible intramedullary nail fixation? J Orthop Surg Res $\underline{6}, 46$

Kaiser MM, Zachert G, Wendlandt R, Eggert R, Stratmann C, Gros N, Schulze-Hessing M, Rapp M (2012): Increasing stability by pre-bending the nails in elastic stabel intramedullary nailing: A biomechanical Analysis of a synthetic femoral spiral fracture model. J Bone Joint Surg 94, 713-718

Kaiser MM, Stratmann C, Zachert G, Schulze-Hessing M, Gros N, Eggert R, Rapp M (2014): Modification of elastic stable intramedullary nailing with a $3^{\text {rd }}$ nail in a femoral spiral fracture model - results of biomechanical testing and a prospective clinical study. BMC Musculoskelet Disord $\underline{15}$, 1-10

Kalkwarf HJ, Laor T, Bean JA (2011): Fracture risk in children with a forearm injury is associated with volumetric bone density and cortical area (by peripheral QCT) and areal bone density (by DXA). Osteoporosis Int 22, 607-616

Kamphaus A, Rapp M, Wessel LM, Buchholz M, massalme E, Schneidmüller D, Roeder C, Kaiser MM (2015): LiLa-Klassifikation für Frakturen der langen Röhrenknochen im Wachstumsalter: Intra- und Interobserverreliabilität. Unfallchirurg 118, 326-335 Keeler K, Dart B, Luhmann SJ, Schoenecker PL, Ortman MR, Dobbs MB, Gordon JE (2009): Antegrade Intramedullary Nailing of Pediatric Femoral Fractures Using an Interlocking Pediatric Femoral Nail and a Lateral Trochanteric Entry Point. J Pediatr Orthop $\underline{29}, 345-351$ 
Kraus R: Technische Komplikationen bei der Versorgung von Frakturen. In: von Laer L (Hrsg.): Das verletzte Kind. Thieme, Stuttgart 2007, 26-32

Kraus R: Epidemiologie. In: Marzi I (Hrsg.): Kindertraumatologie. Springer, Heidelberg 2010, 32-35

Krischak G: Traumatologie für Physiotherapeuten: 13 Tabellen (Physiolehrbuch Krankheitslehre). Thieme, Stuttgart 2005

Lascombes P, Haumont T, Journeau P (2006): Use and abuse of flexible intramedullary nailing in children and adolescents. J Pediatr Orthop 26, 827-834

Lascombes P, Huber H, Fay R, Popkov D, Haumont T, Journeau P (2013): Flexible intramedullary nailing in children: nail to medullary canal diameters optimal ratio. J Pediatr Orthop $\underline{33}, 403-408$

Leventhal JM, Martin KD, Asnes AG (2008): Incidence of fractures attributable to abuse in young hospitalized children: results from analysis of a United States database. Pediatrics $\underline{122}$, 599-604

Lüllmann-Rauch R: Taschenlehrbuch Histologie. 2. Auflage; Thieme, Stuttgart 2006 Maier M, Maier-Heidkamp M, Lehnert M, Wirbel R, Marzi I (2003): Ausheilungsergebnisse konservativ und operativ versorgter kindlicher Femurfrakturen. Unfallchirurg $\underline{106}, 48-54$

Maier M, Schneidmüller D, Marzi I: Oberschenkel. In: Marzi I (Hrsg.): Kindertraumatologie. 2. Auflage; Springer, Heidelberg 2010

Martus JE (2016): Rigid Intramedullary Nailing of Femoral Shaft Fractures for Patients Age 12 and Younger: Indications and Technique. J Child Orthop 36, 35-40

Marzi I: Grundlagen der operativen Frakturbehandlung. In: Dietz HG, Illing P, Schmittenbecher PP, Slongo T, Sommerfeldt DW (Hrsg.): Praxis der Kinder- und Jugendtraumatologie. Springer, Heidelberg 2011, 99-114

McKay HA, Bailey DA, Mirwald RL, Davison KS, Faulkner RA (1998): Peak bone mineral accrual and age at menarche in adolescent girls: A 6-year longitudinal study. J Pediatr $\underline{133}, 682-687$

Metaizeau J-P (2004): Stable elastic intramedullary nailing for fractures of the femur in children. J Bone Joint Surg (Br) $\underline{86}$, 954-957

Moroz LA, Launay F, Kocher MS, Newton PO, Frock SL, Sponseller PD, Flynn JM (2006): Titanium elastic nailing of fractures of the femur in children: Predictors of complications and poor outcome. J Bone Joint Surg $(\mathrm{Br}) \underline{88}$ 
Müller M: Chirurgie für Studium und Praxis: Unter Berücksichtigung des Gegenstandskataloges und der mündlichen Examina in den Ärztlichen Prüfungen 2012/13. 11.

Auflage; Med. Verl.- und Informationsdienste, Breisach am Rhein 2011

Narayanan UG, Hyman JE, Wainwright AM, Rang M, Alman BA (2004): Complications of Elastic Stable Intramedullary Nail Fixation of Pediatric Femoral Fractures, and How to Avoid Them. J Pediatr Orthop, 363-369

Nectoux E, Giacomelli MC, Karger C, Gicquel P, Clavert JM (2008): Use of end caps in elastic stable intramedullary nailing of femoral and tibial unstable fractures in children: preliminary results in 11 fractures. J Child Orthop $\underline{2}$, 309-314

Niethard FU: Kinderorthopädie. 2. Auflage; Thieme, Stuttgart 2010

Niethard FU, Pfeil J, Biberthaler P: Erworbene Wachstumsstörungen. In: Niethard FU, Pfeil J, Biberthaler P (Hrsg.): Orthopädie und Unfallchirurgie (Duale Reihe). 6. Auflage; Thieme, Stuttgart 2009, 118-144

Parfitt AM (1994): The two faces of growth: Benefits and risks to bone integrity. Osteoporosis Int 4, 382-398

Poolman RW, Kocher MS, Bhandari M (2006): Pediatric Femoral Fractures: A Systematic Review of 2422 Cases. J Orthop Trauma 20, 648-654

Prata do Nascimento F, Santilill C, Akkarilll M, Waisberg G, dos Reis Braga S, Maria P (2013): Flexible intramedullary nails with traction versus plaster cast for treating femoral shaft fractures in children: comparative retrospective study. Sao Paulo Med J $\underline{131}, 5-12$

Ramseier LE, Janicki JA, Weir S, Narayanan UG (2010): Femoral Fractures in Adolescents: A Comparison of Four Methods of Fixation. J Bone Joint Surg $\underline{92}, 1122-$ 1129

Raschke MJ, Haas NP: Traumatologie. In: Siewert JR, Stein HJ, Hubert J (Hrsg.):

Chirurgie. 9. Auflage; Springer, Heidelberg 2012, 865-999

Rauch F (2012): The dynamics of bone structure developement during pubertal growth. J Musculoskelet Neuronal Interact 12, 1-6

Rewers A, Hedegaard H, Lezotte D, Meng K, Battan FK, Emery K, Hamman RF (2005): Childhood femur fractures, associated injuries, and sociodemographic risk factors: a population-based study. Pediatrics $\underline{115}$, 543-52 
Reynolds RA, Lagakis JE, Thomas R, Slongo TF, Hunter JB, Clavert J-M (2012):

Intramedullary nails for pediatric diaphyseal femur fractures in older, heavier children: early results. J Child Orthop $\underline{6}, 181-188$

Schalamon J, Dampf S, Singer G, Ainoedhofer H, Petnehazy T, Hoellwarth ME, Saxena AK (2011): Evaluation of fractures in children and adolescents in a Level I Trauma Center in Austria. J Trauma $\underline{71}, 19-25$

Schmittenbecher P: Wachstumsphänomene und Korrekturmechanismen des wachsenden Skeletts. In: Dietz HG, Illing P, Schmittenbecher PP, Slongo T, Sommerfeldt DW (Hrsg.) : Praxis der Kinder- und Jugendtraumatologie. Springer, Heidelberg 2011, 27-53

Sela Y, Hershkovich O, Sher-Lurie N, Schindler A, Givon U (2013): Pediatric femoral shaft fractures: treatment strategies according to age -13 years of experience in one medical center. J Orthop Surg Res $\underline{8}, 23$

Sink EL, Gralla J, Repine M (2005): Complications of Pediatric Femur Fractures Treated With Titanium Elastic Nails. J Pediatr Orthop 25, 577-580

Slongo T, Audigé L, AO Pediatric Classification Group (2007) (Hrsg.): AO Pediatric Comprehensive Classification of Long-Bone Fractures (PCFF), AO Foundation, Davos, 2010

Slongo T, Audigé L, Schneidmüller D, von Laer L: Frakturklassifikation im Kindesalter. In: Marzi I (Hrsg.): Kindertraumatologie. 2. Auflage; Springer, Heidelberg 2010, 19-30 Strohm PC, Schmittenbecher PP (2015): Femoral shaft fractures in children under 3 years old. Current treatment standard. Unfallchirurg $\underline{118}, 48-52$

Tomaszewski R, Gap A (2014): Results of the treatment of the open femoral shaft fractures in children. J Orthop $\underline{11}, 78-81$

Vogel L: Frakturen im Wachstumsalter - Vergleich der LiLa-Klassifikation nach von Laer mit der AO-Klassifikation nach Slongo. Med.Diss. Erlangen-Nürnberg 2012 von Heideken J, Svensson T, Blomqvist P, Haglund-Akerlind Y, Janarv P-M (2011): Incidence and trends in femur shaft fractures in Swedish children between 1987 and 2005. J Pediatr Orthop $\underline{31}$, 512-519

von Laer L: Verletzungsformen. In: Marzi I (Hrsg.): Kindertraumatologie. 2. Auflage: Springer, Heidelberg 2010 
von Laer L, Worel AM, Röder C, Staub LP, Busato A (2005): Qualitätssicherung in der Traumatologie des Wachstumsalters - eine Pilotstudie. 1-15. http://www.lila.org/content/index.php?id=21, siehe Internetquellen

von Laer L, Kraus R, Linhart W: Frakturen und Luxationen im Wachstumsalter. 5. Auflage; Thieme, Stuttgart 2007 von Laer L, Kraus R, Linhart WE: Frakturen und Luxationen im Wachstumsalter. 6. Auflage; Thieme, Stuttgart 2013

Wang Q, Cheng S, Alen M, Seeman E (2009): Bone's structural diversity in adult females is established before puberty. J Clin Endocrinol Metab 94, 1555-1561

Weinberg AM, Schneidmüller D: Frakturklassifikation. In: Weinberg AM, Schneidmüller D (Hrsg.) : Unfallchirurgie bei Kindern: Kompendium der Kindertraumatologie.

Deutscher Ärzte-Verlag, Köln 2010a, 1-6

Weinberg AM, Schneidmüller D: Oberschenkel. In: Weinberg AM, Schneidmüller D (Hrsg.) : Unfallchirurgie bei Kindern: Kompendium der Kindertraumatologie. Deutscher Ärzte-Verlag, Köln 2010b, 175-190

Worel AM, Slongo T: Behandlungsprinzipien. In: Marzi I (Hrsg.): Kindertraumatologie. 2. Auflage; Springer, Heidelberg 2010, 49-75

Xu L, Nicholson P, Wang Q, Alen M, Cheng S (2009): Bone and muscle development during puberty in girls: a seven-year longitudinal study. J Bone Mineral Res 24, 16931698

Zimmermann B: Behandlung der kindlichen Femurfraktur mit dem Elastisch Stabilen Intramedullären Nagel (ESIN): Klinisch funktionelle Langzeituntersuchung. Med. Diss. Köln 2008 


\subsection{Bild-, Internet-, Tabellen- und sonstige Quellen}

\begin{tabular}{|c|c|}
\hline Abbildung & Quelle \\
\hline 1 & $\begin{array}{l}\text { Dietz HG: Verletzungen des Femurschafts im Kindesalter. In: Haas NP, Krettek H } \\
\text { (Hrsg.): Tscherne Unfallchirurgie, Hüfte und Oberschenkel. Springer, Heidelberg 2012, } \\
\text { 319, Abb. } 10.1 \\
\text { Beschriftung modifiziert. } \\
\text { Die Verwendung erfolgt mit Genehmigung des Springer-Verlags. }\end{array}$ \\
\hline 2 & $\begin{array}{l}\text { http://www.li-la.org/content/index.php?id=21, } \quad \text { Zugriff } \quad \text { am } \quad 30.01 .2017 \\
\text { Die Verwendung erfolgt mit Genehmigung des Li-La e.V.s. }\end{array}$ \\
\hline 3 & $\begin{array}{l}\text { Slongo T, Audigé L, Schneidmüller D, von Laer L: Frakturklassifikation im Kindesalter. } \\
\text { In: Marzi I (Hrsg.): Kindertraumatologie. 2. Auflage; Springer, Heidelberg 2010, 21, } \\
\text { Abb. } 3.2 \\
\text { Die Verwendung erfolgt mit Genehmigung des Springer-Verlags. }\end{array}$ \\
\hline 4 & $\begin{array}{l}\text { http://www.li-la.org/content/index.php?id=21, Zugriff } \quad \text { am } \quad 01.10 .2016 \\
\text { Die Verwendung erfolgt mit Genehmigung des Li-La e.V.s. }\end{array}$ \\
\hline 5 & $\begin{array}{l}\text { Dietz HG: Verletzungen des Femurschafts im Kindesalter. In: Haas NP, Krettek H } \\
\text { (Hrsg.): Tscherne Unfallchirurgie, Hüfte und Oberschenkel. Springer, Heidelberg 2012, } \\
\text { 322, Abb. } 10.2 \\
\text { Die Verwendung erfolgt mit Genehmigung des Springer-Verlags. }\end{array}$ \\
\hline 6 & $\begin{array}{l}\text { Maier M, Schneidmüller D, Marzi I: Oberschenkel. In: Marzi I (Hrsg.): Kindertrauma- } \\
\text { tologie. 2. Auflage; Springer, Heidelberg 2010, 271, Abb. } 19.5 \\
\text { Die Verwendung erfolgt mit Genehmigung des Springer-Verlags. }\end{array}$ \\
\hline 7 & $\begin{array}{l}\text { Dietz HG: Verletzungen des Femurschafts im Kindesalter. In: Haas NP, Krettek H } \\
\text { (Hrsg.): Tscherne Unfallchirurgie, Hüfte und Oberschenkel. Springer, Heidelberg 2012, } \\
\text { 323, Abb. } 10.4 \\
\text { Die Verwendung erfolgt mit Genehmigung des Springer-Verlags. }\end{array}$ \\
\hline 8 & $\begin{array}{l}\text { Maier M, Schneidmüller D, Marzi I: Oberschenkel. In: Marzi I (Hrsg.): Kindertrauma- } \\
\text { tologie. 2. Auflage; Springer, Heidelberg 2010, 271, Abb. } 19.4 \\
\text { Die Verwendung erfolgt mit Genehmigung des Springer-Verlags. }\end{array}$ \\
\hline 9 & $\begin{array}{l}\text { Maier M, Schneidmüller D, Marzi I: Oberschenkel. In: Marzi I (Hrsg.): Kindertrauma- } \\
\text { tologie. 2. Auflage; Springer, Heidelberg 2010, 271, Abb. } 19.4 \\
\text { Die Verwendung erfolgt mit Genehmigung des Springer-Verlags. }\end{array}$ \\
\hline 10 & $\begin{array}{l}\text { Dietz HG: Verletzungen des Femurschafts im Kindesalter. In: Haas NP, Krettek H } \\
\text { (Hrsg.): Tscherne Unfallchirurgie, Hüfte und Oberschenkel. Springer, Heidelberg 2012, } \\
\text { 324, Abb. } 10.5 \\
\text { Die Verwendung erfolgt mit Genehmigung des Springer-Verlags. }\end{array}$ \\
\hline
\end{tabular}




\begin{tabular}{|c|c|}
\hline 11 & $\begin{array}{l}\text { Dietz HG: Verletzungen des Femurschafts im Kindesalter. In: Haas NP, Krettek H } \\
\text { (Hrsg.): Tscherne Unfallchirurgie, Hüfte und Oberschenkel. Springer, Heidelberg 2012, } \\
\text { 324, Abb. } 10.7 \\
\text { Die Verwendung erfolgt mit Genehmigung des Springer-Verlags. }\end{array}$ \\
\hline 12 & $\begin{array}{l}\text { Dietz HG: Verletzungen des Femurschafts im Kindesalter. In: Haas NP, Krettek H } \\
\text { (Hrsg.): Tscherne Unfallchirurgie, Hüfte und Oberschenkel. Springer, Heidelberg 2012, } \\
\text { 330, Abb. } 10.11 \\
\text { Die Verwendung erfolgt mit Genehmigung des Springer-Verlags. }\end{array}$ \\
\hline 13 & $\begin{array}{l}\text { Dietz HG: Verletzungen des Femurschafts im Kindesalter. In: Haas NP, Krettek H } \\
\text { (Hrsg.): Tscherne Unfallchirurgie, Hüfte und Oberschenkel. Springer, Heidelberg 2012, } \\
\text { 327, Abb. } 10.8 \\
\text { Die Verwendung erfolgt mit Genehmigung des Springer-Verlags. }\end{array}$ \\
\hline 14 & $\begin{array}{l}\text { Gresing T: Hüftgelenk und Oberschenkel. In: Dietz HG, Illing P, Schmittenbecher PP, } \\
\text { Slongo T, Sommerfeld DW (Hrsg.): Praxis der Kinder- und Jugendtraumatologie. } \\
\text { Springer, Heidelberg 2011, 369, Abb. } 19.16 \\
\text { Die Verwendung erfolgt mit Genehmigung des Springer-Verlags. }\end{array}$ \\
\hline 15 & $\begin{array}{l}\text { Raschke MJ, Haas NP: Unfallchirurgie. In: Siewert JR, Stein HJ, Hubert J (Hrsg.): } \\
\text { Chirurgie mit integriertem Fallquiz. 9. Auflage, Springer, Heidelberg 2012, 878, Abb. } \\
8.14 \\
\text { Die Verwendung erfolgt mit Genehmigung des Springer-Verlags. }\end{array}$ \\
\hline 16 & $\begin{array}{l}\text { Dietz HG: Verletzungen des Femurschafts im Kindesalter. In: Haas NP, Krettek H } \\
\text { (Hrsg.): Tscherne Unfallchirurgie, Hüfte und Oberschenkel. Springer, Heidelberg 2012, } \\
\text { 325, Abb. } 10.6 \\
\text { Die Verwendung erfolgt mit Genehmigung des Springer-Verlags. }\end{array}$ \\
\hline 17 & $\begin{array}{l}\text { von Laer L: Knochenwachstum und Knochenheilung. In: Marzi I (Hrsg.): Kindertrauma- } \\
\text { tologie. 2. Auflage, Springer, Heidelberg 2010, 8, Abb. } 1.12 \\
\text { Die Verwendung erfolgt mit Genehmigung des Springer-Verlags. }\end{array}$ \\
\hline 18 & $\begin{array}{l}\text { von Laer L: Knochenwachstum und Knochenheilung. In: Marzi I (Hrsg.): Kindertrauma- } \\
\text { tologie. 2. Auflage, Springer, Heidelberg 2010, 8, Abb. } 1.13 \\
\text { Die Verwendung erfolgt mit Genehmigung des Springer-Verlags. }\end{array}$ \\
\hline 19 & $\begin{array}{l}\text { von Laer L: Knochenwachstum und Knochenheilung. In: Marzi I (Hrsg.): Kindertrauma- } \\
\text { tologie. 2. Auflage, Springer, Heidelberg 2010, 2, Abb. } 1.2 \\
\text { Die Verwendung erfolgt mit Genehmigung des Springer-Verlags. }\end{array}$ \\
\hline 20 & $\begin{array}{l}\text { von Laer L: Knochenwachstum und Knochenheilung. In: Marzi I (Hrsg.): Kindertrauma- } \\
\text { tologie. 2. Auflage, Springer, Heidelberg 2010, 9, Abb. } 1.14 \\
\text { Die Verwendung erfolgt mit Genehmigung des Springer-Verlags. }\end{array}$ \\
\hline
\end{tabular}




\begin{tabular}{|l|l|}
\hline 21 & $\begin{array}{l}\text { Biberthaler P, Niethard FU, Pfeil J: Duale Reihe Orthopädie und Unfallchirurgie. } 7 . \\
\text { Auflage, Thieme, Stuttgart 2014, 138, Abb. B-2.16 } \\
\text { Die Verwendung erfolgt mit Genehmigung des Thieme-Verlages. }\end{array}$ \\
\hline 45 & $\begin{array}{l}\text { Slongo T, Audigé L, AO Pediatric Classification Group (2007): AO Pediatric Comprehen- } \\
\text { sive Classification of Long-Bone Fractures (PCCF). AO Foundation, Davos 2010, } 6\end{array}$ \\
\hline 46 & $\begin{array}{l}\text { Slongo T, Audigé L, AO Pediatric Classification Group (2007): AO Pediatric Comprehen- } \\
\text { sive Classification of Long-Bone Fractures (PCCF). AO Foundation, Davos 2010, } 25\end{array}$ \\
\hline
\end{tabular}

\begin{tabular}{|l|l|}
\hline Internetquelle & Zugriff am \\
\hline http://www.awmf.org/leitlinien/detail/ll/006-016.html & 15.08 .2016 \\
\hline http://www.li-la.org/content/index.php?id=2 & 13.07 .2016 \\
\hline http://www.li-la.org/content/index.php?id=21 & 20.07 .2016 \\
\hline
\end{tabular}

\begin{tabular}{|l|l|}
\hline Tabellennummer & Quelle \\
\hline 1 & $\begin{array}{l}\text { Modifiziert nach: Schneidmüller D, Weinberg A: Frakturklassifikationen. In: } \\
\text { Weinberg AM, Schneidmüller D (Hrsg.): Unfallchirurgie bei Kindern: Kompendium } \\
\text { der Kindertraumatologie; Deutscher Ärzte-Verlag, Köln 2010, 2-4 }\end{array}$ \\
\hline 2 & $\begin{array}{l}\text { Modifiziert nach: Von Laer L, Kraus R, Linhart WE: Frakturen und Luxationen im } \\
\text { Wachstumsalter, 5. Auflage; Thieme, Stuttgart 2007, 280-281 }\end{array}$ \\
\hline 14 & $\begin{array}{l}\text { Modifiziert nach: Gresing T: Hüftgelenk und Oberschenkel. In: Dietz HG, Illing P, } \\
\text { Schmittenbecher PP, Slongo T, Sommerfeldt DW (Hrsg.): Praxis der Kinder- und } \\
\text { Jugendtraumatologie; Springer-Verlag Berlin 2011, 363 }\end{array}$ \\
\hline
\end{tabular}

\begin{tabular}{|l|l|}
\hline Art der Quelle & Titel \\
\hline Video & $\begin{array}{l}\text { AO Teaching Video on DVD. In: Slongo T, Wilkins KE: AO Manual of Fracture } \\
\text { Management. Elastic Stable Intramedullary Nailing (ESIN) in Children; Thieme, } \\
\text { Stuttgart 2006 }\end{array}$ \\
\hline
\end{tabular}




\section{Danksagung}

Bei der Konzeption und Realisierung der vorliegenden Arbeit habe ich umfangreiche Unterstützung erfahren, für die ich mich an dieser Stelle herzlich bedanken möchte.

Mein besonderer Dank gilt Herrn Professor Stephan Sehmisch für die fachliche Betreuung meines Promotionsvorhabens. Seine Anregungen, ermutigenden Kommentare und wertvollen Rückmeldungen erleichterten mir das Anfertigen der Promotionsschrift sehr.

Ganz herzlich danken möchte ich Herrn Privatdozent Stefan Piatek von der unfallchirurgischen Klinik sowie Herrn Dr. med. Hardy Krause von der Abteilung für Kinderchirurgie und Kindertraumatologie am Universitätsklinikum Magdeburg für die freundliche Zusammenarbeit und die Bereitstellung der Patient(inn)endaten.

Dr. Asmus Zoch danke ich für seine Geduld, die Diskussionen zu statistischen Tests und die Überprüfung der statistischen Auswertung meiner Daten.

Außerdem möchte ich Simon Schallenberg und Sophia Westpfahl meinen Dank aussprechen für das Zusenden benötigter Literatur aus den Universitätsbibliotheken Köln und Rostock. 


\section{Lebenslauf}

Am 3. Oktober 1988 wurde ich in Berlin als Tochter von Rita und Fritz Klauser geboren. Im Alter von vier Jahren zog ich mit meinen Eltern nach Göttingen und wurde im Sommer 1995 eingeschult. Ab der siebten Klasse besuchte ich den mathematischnaturwissenschaftliche Zweig des Felix-Klein-Gymnasium. Sechs Monate der elften Klasse absolvierte ich in Südafrika an der Rhenish Girls' High School in Stellenbosch. Im Anschluss besuchte ich die Oberstufe des Theodor-Heuss-Gymnasiums, wo ich 2008 mein Abitur erwarb.

Es folgte ein Praktikumsaufenthalt als Volunteer in der Notaufnahme des Hottentots Holland Hospitals, eines staatlichen Krankenhauses in Somerset West, Südafrika. Die Erfahrungen dort prägten und motivierten mich für meine Berufswahl, sodass ich mich anschließend auf einen Studienplatz der Humanmedizin bewarb. Die Universität Göttingen bat mir nach einem Auswahlgespräch einen Platz für das Sommersemester 2009 an. Zwei Jahre später legte ich dort erfolgreich das Physikum ab. Ab 2012 arbeitete ich als Rufdienststudentin der unfallchirurgischen Notaufnahme am Universitätsklinikum Göttingen, wodurch mein Interesse für dieses Fachgebiet maßgeblich geweckt wurde. Im Rahmen eines Erasmus-Stipendiums studierte ich 2013 acht Monate an der Universität La Sapienza in Rom. Im November des gleichen Jahres wurde ich Promovendin in der Abteilung für Unfallchirurgie, Plastische und Wiederherstellungschirurgie der Universitätsklinik Göttingen, betreut durch Herrn Professor Stephan Sehmisch, und begann mit der Datenerhebung für die vorliegende Arbeit. 2014 bestand ich das zweite Staatsexamen. Anschließend sammelte ich Erfahrungen in zwei Krankenhäusern Göttingens (Pathologie, Unfallchirurgie und Geriatrie) sowie in Liverpool (Infektions- und Tropenkrankheiten) und Kigali, Ruanda, (Allgemeinchirurgie und Geburtshilfe). Im November 2015 bestand ich die Dritte Ärztliche Prüfung und erhielt die Approbation. Meine erste Arbeitsstelle trat ich im November 2016 in der geriatrischen Abteilung des Evangelischen Krankenhauses Göttingen-Weende an. 